\title{
A PRAGMATIC PILOT STUDY OF COGNITIVE BEHAVIOURAL THERAPY FOR INSOMNIA AMONG PEOPLE LIVING WITH HIV
}

\author{
by \\ Tyler Gordon Tulloch \\ Master of Arts in Psychology, Ryerson University, 2013 \\ Bachelor of Arts Honours in Psychology, Concordia University, 2009 \\ Bachelor of Arts Honours in Sociology, York University, 2003 \\ Associate of Arts in General Studies, Briercrest College, 1999 \\ A dissertation \\ presented to Ryerson University \\ in partial fulfillment of the requirements for the degree of \\ Doctor of Philosophy \\ in the program of Psychology
}

Toronto, Ontario, Canada, 2019

(C) Tyler Gordon Tulloch, 2019 


\section{AUTHOR'S DECLARATION FOR SUBMISSION OF A DISSERTATION}

I hereby declare that I am the sole author of this dissertation. This is a true copy of the dissertation, including any required final revisions, as accepted by my examiners.

I authorize Ryerson University to lend this dissertation to other institutions or individuals for the purpose of scholarly research.

I further authorize Ryerson University to reproduce this dissertation by photocopying or by other means, in total or in part, at the request of other institutions or individuals for the purpose of scholarly research.

I understand that my dissertation may be made electronically available to the public. 
A Pragmatic Pilot Study of Cognitive Behavioural Therapy for Insomnia among People Living with HIV

Doctor of Philosophy in Psychology, 2019

Tyler Gordon Tulloch

Psychology, Ryerson University

\begin{abstract}
The prevalence of insomnia among people living with HIV (PWH) is considerably higher than in the general population. Cognitive, behavioural, and biopsychosocial explanations for this elevated prevalence have been proposed, but there is a lack of consensus in the literature. Sleep disturbance is associated with disrupted immune functioning at the cellular level and increased risk of contracting infectious diseases; therefore, insomnia may be particularly problematic for PWH. Cognitive behavioural therapy for insomnia (CBT-I) is the first-line treatment for insomnia, and is effective at treating insomnia among individuals with comorbid medical disorders. Surprisingly, no study has examined its efficacy among PWH. This pragmatic pilot study examined features of insomnia among $48 \mathrm{PWH}$ and was the first study to examine sleep disturbance among PWH using American Academy of Sleep Medicine guidelines for evaluating chronic insomnia. On average, insomnia chronicity was 8.2 years and was of moderate severity. Psychiatric comorbidity was common, as were comorbid sleep disorders. Insomnia severity was associated with psychosocial variables including sleep effort, self-efficacy for sleep, depression, anxiety, stress, and social functioning-related quality of life. This study was also the first to examine safety, feasibility, acceptability, and intervention effects of CBT-I. These were examined among a subsample of $10 \mathrm{PWH}$ using single-case interrupted time-series design. Thematic analysis was used to explore participant perceptions of CBT-I. Large effect sizes were
\end{abstract}


observed for improvement in insomnia severity, sleep efficiency, and total wake time. Reliable and clinically significant reduction in insomnia severity was observed for all but one participant, and half met criteria for insomnia remission. Overall, CBT-I was an efficacious treatment for insomnia, and participant feedback was consistent with quantitative results. Participants viewed CBT-I as a safe and acceptable treatment for insomnia. Participant feedback identified preexisting needs, positive impacts and challenges of CBT-I, perceived mechanisms of change, and suggested modifications to tailor CBT-I for PWH. Given this preliminary support for the efficacy of CBT-I among PWH, future research should seek to demonstrate generalizability of these effects. Partnering with HIV community organizations may be important next step in conducting future research and increasing accessibility of CBT-I to PWH.

Keywords: HIV, insomnia, cognitive behavioural therapy, pilot study, pragmatic trial, thematic analysis 


\section{Acknowledgements}

This dissertation represents the culmination of many years of hard work, late nights, personal sacrifices, and countless cups of coffee. This was not a solo endeavor and would not have been possible without the support of a number of key people. First, I would like to extend my deepest gratitude to my supervisor, Dr. Trevor Hart. Your support was unwavering right from my first day as a not-so-young master's student in the HIV Prevention Lab. You encouraged my academic curiosity and provided valuable opportunities for me to expand my research and clinical skills and liaise with colleagues near and far. Your support was critical in the formation and completion of this project, and in my development as a scientist-practitioner. Thank you to Dr. Colleen Carney for piquing my interest in sleep disorders and for your invaluable role in training me in the ways of cognitive behavioural therapy for insomnia. Thank you to Dr. Kelly McShane for my initiation into the world of qualitative research, which I have found to be a crucial addition to my research toolbox, and which I will carry forward with me. Thank you to all the participants of this study, without whom this study would not have been possible. I would also like to extend my heartfelt gratitude to my fellow graduate students in the HIV Prevention Lab_-Anne, Amrita, and Danielle for showing me the ropes, and Natalie, Marie and Ammaar for joining me on this wild journey. We have shared much more than office space in the many years we have known each other, and your company has been delightful.

As important as the contributions of my professional colleagues and supervisors have been, I could never have accomplished this endeavour without the unfailing support of loved ones. Ralphie, your patience and numerous sacrifices have not gone unnoticed, and for them, I thank you from the bottom of my heart. I look forward to starting a new phase of our life together, hopefully one with me spending a little less time shut away in the office. The 
unwavering love and support of family, especially mom and dad, has provided a strong foundation on which to stand and has played an integral role not only in the completion of this dissertation, but also in my achievement of many important goals in life. Thank you so very much. I would like to thank all of my friends who have not seen much of me in the past eight years-I plan to make up for my absence soon! Finally, I would like to thank my four-legged friend, Maurice, who started out on this journey with me, but didn't make it all the way to the end. Your love and loyalty were felt every day and you will be forever missed. 


\section{Dedication}

This work is dedicated to the millions of people whose lives have been impacted by HIV and AIDS. 


\section{Table of Contents}

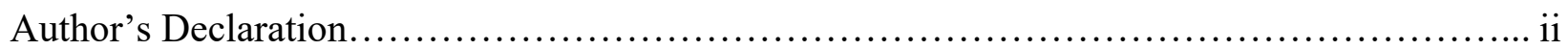

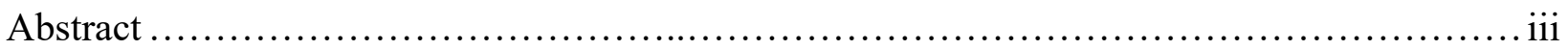

Acknowledgements...................................................................

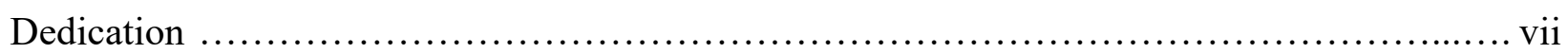

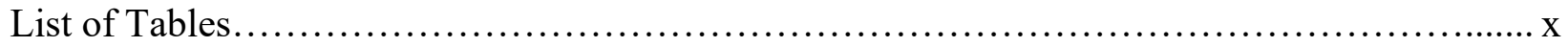

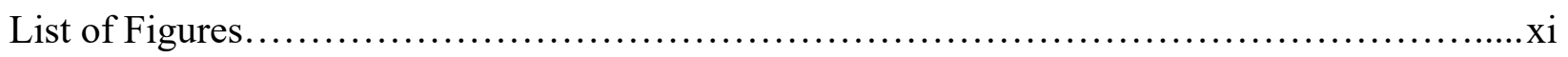

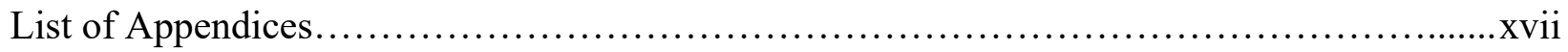

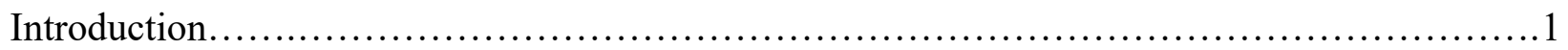

Insomnia Disorder........................................................... 1

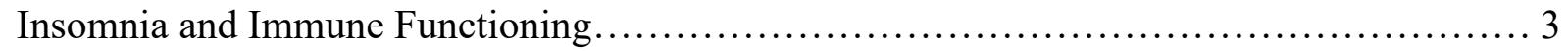

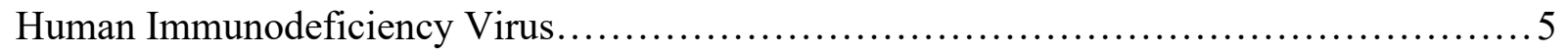

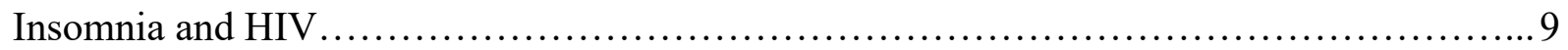

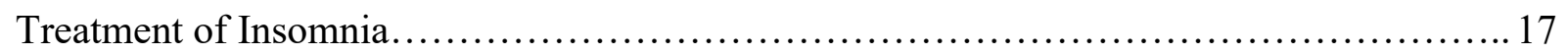

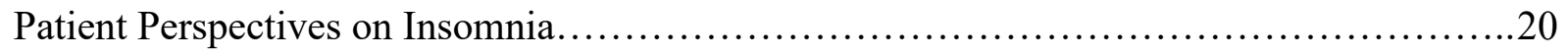

Current Study .............................................................. 22

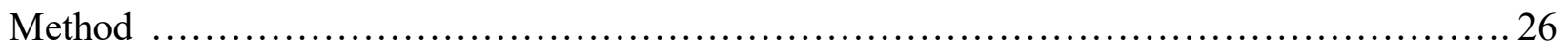

Participants.................................................................. 26

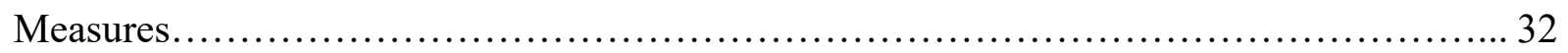

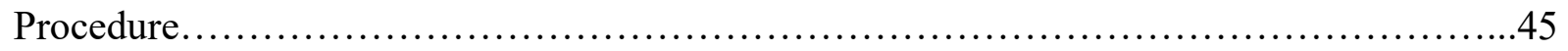

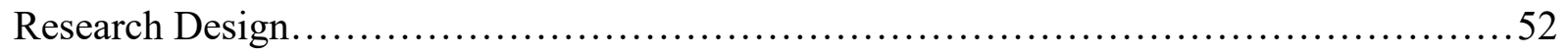

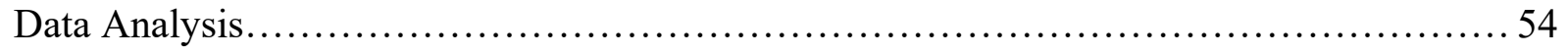

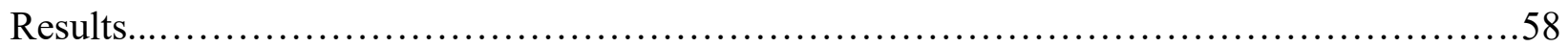




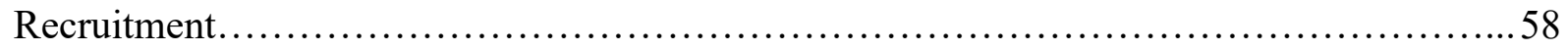

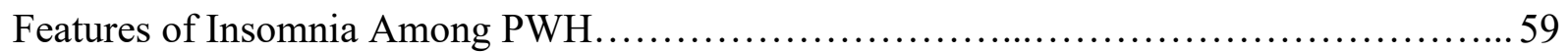

Association Between HIV- and Sleep-Related Variables................................. 70

Summary of Results Exploring Features of Insomnia among PWH.......................74

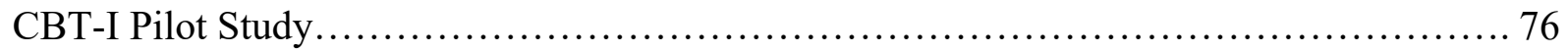

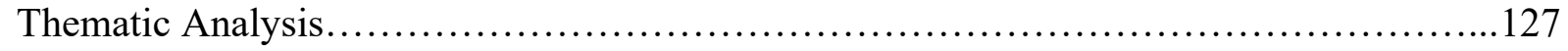

Summary of Results for CBT-I Pilot Study ........................................ 154

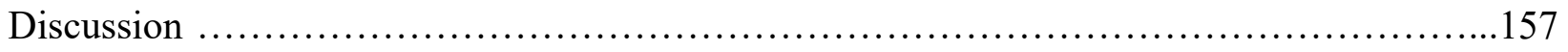

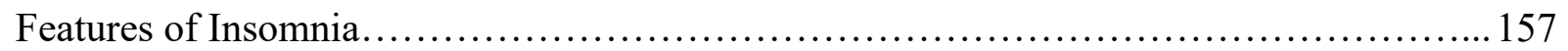

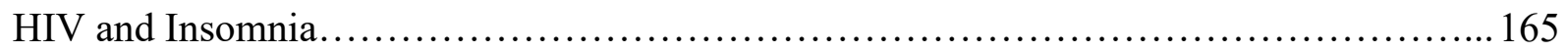

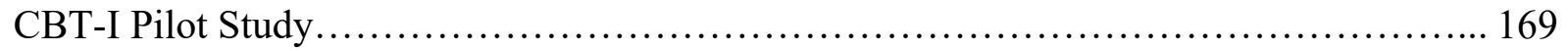

Participant Perceptions of CBT-I.................................................. 179

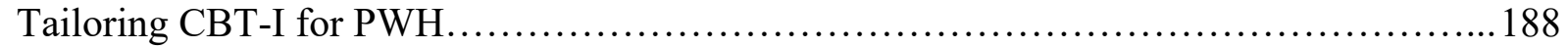

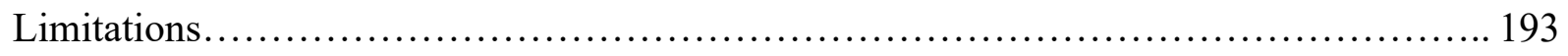

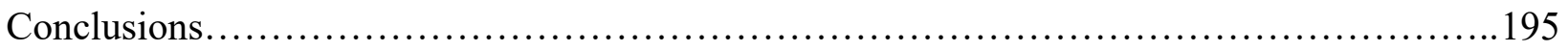

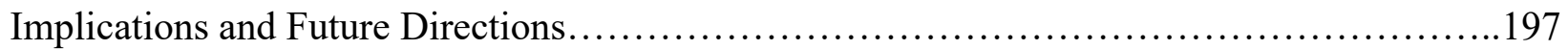

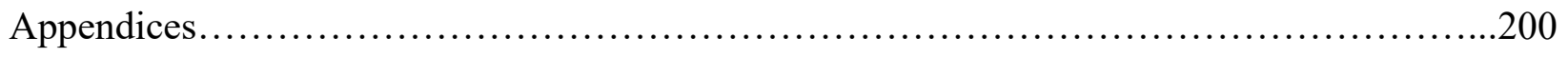

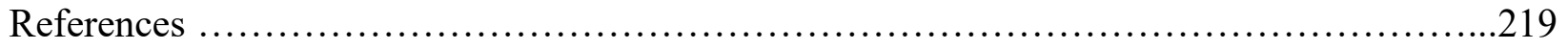

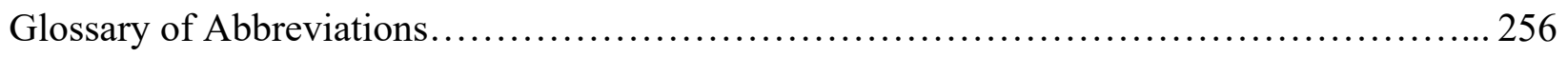




\section{List of Tables}

Table 1. Demographic Variables in the Full Sample and the Pilot Study Subsample........... 30

Table 2. Clinical Variables of Full Sample at Baseline....................................63

Table 3. Spearman Correlations Between Indicators of Insomnia and Clinical Variables........ 69

Table 4. Clinical Characteristics of Pilot Study Participants Pre- and Post-CBT-I............ 77

Table 5. Spearman Correlations Between Baseline to Posttreatment Symptom Severity

Change Scores...................................................... 119 


\section{List of Figures}

Figure 1. Recruitment response rate and flow of participants through the study........ 60

Figure 2. Number of comorbid psychiatric disorders (non-sleep and sleep) diagnosed at baseline 66

Figure 3. Frequency of comorbid psychiatric disorders at baseline....

Figure 4a. Mean Insomnia Severity Index scores with baseline period mean $\pm 2 S D$ plotted...8 80

Figure 4b. Mean Insomnia Severity Index scores with baseline and intervention period celeration lines plotted. 80

Figure 5a. Participant P1 Insomnia Severity Index scores with baseline period mean $\pm 2 S D$

plotted.

Figure 5b. Participant P1 Insomnia Severity Index scores with baseline and intervention period celeration lines plotted

Figure 6a. Participant P2 Insomnia Severity Index scores with baseline period mean $\pm 2 S D$ plotted.....

Figure 6b. Participant P2 Insomnia Severity Index scores with baseline and intervention period celeration lines plotted.

Figure 7a. Participant P3 Insomnia Severity Index scores with baseline period mean $\pm 2 S D$ plotted.

Figure 7b. Participant P3 Insomnia Severity Index scores with baseline and intervention period celeration lines plotted 84

Figure 8a. Participant P4 Insomnia Severity Index scores with baseline period mean $\pm 2 S D$

plotted. 85 
Figure 8b. Participant P4 Insomnia Severity Index scores with baseline and intervention period celeration lines plotted.

Figure 9a. Participant P5 Insomnia Severity Index scores with baseline period mean $\pm 2 S D$ plotted. 86

Figure 9b. Participant P5 Insomnia Severity Index scores with baseline and intervention period celeration lines plotted. 86

Figure 10a. Participant P6 Insomnia Severity Index scores with baseline period mean $\pm 2 S D$

plotted.

Figure 10b. Participant P6 Insomnia Severity Index scores with baseline and intervention period celeration lines plotted

Figure 11a. Participant P7 Insomnia Severity Index scores with baseline period mean $\pm 2 S D$ plotted 88

Figure 11b. Participant P7 Insomnia Severity Index scores with baseline and intervention period celeration lines plotted 88

Figure 12a. Participant P8 Insomnia Severity Index scores with baseline period mean $\pm 2 S D$ plotted

Figure 12b. Participant P8 Insomnia Severity Index scores with baseline and intervention period celeration lines plotted.

Figure 13a. Participant P9 Insomnia Severity Index scores with baseline period mean $\pm 2 S D$ plotted..... 90

Figure 13b. Participant P9 Insomnia Severity Index scores with baseline and intervention period celeration lines plotted 90 
Figure 14a. Participant P10 Insomnia Severity Index scores with baseline period mean $\pm 2 S D$

plotted.....

Figure 14b. Participant P10 Insomnia Severity Index scores with baseline and intervention period celeration lines plotted............................................. 91

Figure 15a. Mean sleep efficiency with baseline period mean $\pm 2 S D$ plotted.................93

Figure 15b. Mean sleep efficiency with baseline and intervention period celeration lines plotted..... 93

Figure 16a. Participant P1 sleep efficiency with baseline period mean $\pm 2 S D$ plotted. .95

Figure 16b. Participant P1 sleep efficiency with baseline and intervention period celeration lines

plotted. 95

Figure 17a. Participant P2 sleep efficiency with baseline period mean $\pm 2 S D$ plotted. 96

Figure 17b. Participant P2 sleep efficiency with baseline and intervention period celeration lines plotted..... 96

Figure 18a. Participant P3 sleep efficiency with baseline period mean $\pm 2 S D$ plotted. 97

Figure 18b. Participant P3 sleep efficiency with baseline and intervention period celeration lines plotted 97

Figure 19a. Participant P4 sleep efficiency with baseline period mean $\pm 2 S D$ plotted. 98

Figure 19b. Participant P4 sleep efficiency with baseline and intervention period celeration lines plotted 98

Figure 20a. Participant P5 sleep efficiency with baseline period mean $\pm 2 S D$ plotted. 99 Figure 20b. Participant P5 sleep efficiency with baseline and intervention period celeration lines plotted. 99

Figure 21a. Participant P6 sleep efficiency with baseline period mean $\pm 2 S D$ plotted. 100 
Figure 21b. Participant P6 sleep efficiency with baseline and intervention period celeration lines plotted 100

Figure 22a. Participant P7 sleep efficiency with baseline period mean $\pm 2 S D$ plotted. .101

Figure 22b. Participant P7 sleep efficiency with baseline and intervention period celeration lines

plotted 101

Figure 23a. Participant P8 sleep efficiency with baseline period mean $\pm 2 S D$ plotted. 102

Figure 23b. Participant P8 sleep efficiency with baseline and intervention period celeration lines plotted. 102

Figure 24a. Participant P9 sleep efficiency with baseline period mean $\pm 2 S D$ plotted. 103

Figure 24b. Participant P9 sleep efficiency with baseline and intervention period celeration lines plotted..... 103

Figure 25a. Participant P10 sleep efficiency with baseline period mean $\pm 2 S D$ plotted. 104

Figure 25b. Participant P10 sleep efficiency with baseline and intervention period celeration lines plotted. 104

Figure 26a. Mean total wake time with baseline period mean $\pm 2 S D$ plotted. 106

Figure 26b. Mean total wake time with baseline and intervention period celeration lines

plotted. 106

Figure 27a. Participant P1 total wake time with baseline period mean $\pm 2 S D$ plotted 107

Figure 27b. Participant P1 total wake time with baseline and intervention period celeration lines plotted. 107

Figure 28a. Participant P2 total wake time with baseline period mean $\pm 2 S D$ plotted. 108 Figure 28b. Participant P2 total wake time with baseline and intervention period celeration lines plotted. 108 
Figure 29a. Participant P3 total wake time with baseline period mean $\pm 2 S D$ plotted

Figure 29b. Participant P3 total wake time with baseline and intervention period celeration lines

plotted 109

Figure 30a. Participant P4 total wake time with baseline period mean $\pm 2 S D$ plotted.

Figure 30b. Participant P4 total wake time with baseline and intervention period celeration lines plotted.....

Figure 31a. Participant P5 total wake time with baseline period mean $\pm 2 S D$ plotted.

Figure 31b. Participant P5 total wake time with baseline and intervention period celeration lines plotted..... 111

Figure 32a. Participant P6 total wake time with baseline period mean $\pm 2 S D$ plotted

Figure 32b. Participant P6 total wake time with baseline and intervention period celeration lines plotted

Figure 33a. Participant P7 total wake time with baseline period mean $\pm 2 S D$ plotted

Figure 33b. Participant P7 total wake time with baseline and intervention period celeration lines

plotted.....

Figure 34a. Participant P8 total wake time with baseline period mean $\pm 2 S D$ plotted

Figure 34b. Participant P8 total wake time with baseline and intervention period celeration lines plotted 114

Figure 35a. Participant P9 total wake time with baseline period mean $\pm 2 S D$ plotted.

Figure 35b. Participant P9 total wake time with baseline and intervention period celeration lines plotted. 115

Figure 36a. Participant P10 total wake time with baseline period mean $\pm 2 S D$ plotted 116 
Figure 36b. Participant P10 total wake time with baseline and intervention period celeration lines

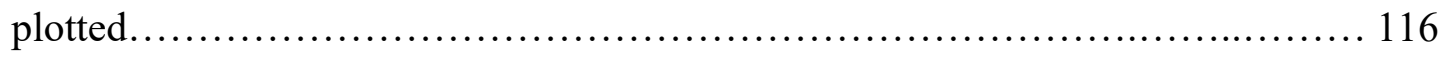

Figure 37. Thematic map of participant perceptions of cognitive behavioural therapy for

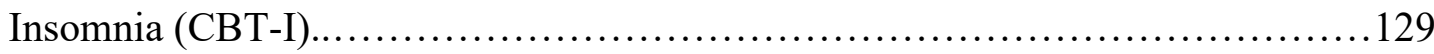

Figure 38. Recommendations for tailoring cognitive behavioural therapy for insomnia (CBT-I) for people living with HIV ................................................. 189 


\section{List of Appendices}

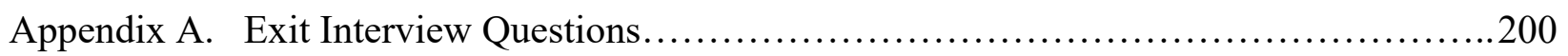

Appendix B. Study Measures................................................ 201 


\section{Introduction}

\section{Insomnia Disorder}

Sleep is essential for optimal psychological and physiological functioning, and reduced sleep quality may compromise the above functions (Robotham, 2011). Insomnia is characterized by dissatisfaction with sleep quantity or quality, accompanied by difficulty initiating and/or maintaining sleep, and/or early morning awakening with inability to fall back asleep (American Psychiatric Association, 2013). For a diagnosis of insomnia disorder according to Diagnostic and Statistical Manual of Mental Disorders, fifth edition (DSM-5; American Psychiatric Association, 2013) criteria, the sleep disturbance must cause clinically significant distress or impairment in some important area of functioning, must occur at least three nights per week for at least the past three months, and must occur despite adequate opportunity for sleep. Furthermore, the sleep disturbance must not be better explained by another sleep-wake disorder such as obstructive sleep apnea (OSA) or circadian rhythm sleep-wake disorder, must not be attributable to the physiological effects of a substance, and must not be explained by other mental disorders or medical conditions (American Psychiatric Association, 2013).

The above DSM-5 diagnostic criteria represent a change from the Diagnostic and Statistical Manual of Mental Disorders, fourth edition, text revision (DSM-IV-TR; American Psychiatric Association, 2000) criteria in several ways. The DSM-5 criteria specify that the sleep disturbance must occur at least three nights per week for at least three months, whereas the DSM-IV criteria specify that the sleep disturbance must occur for at least one month, with no specified frequency. Additionally, the DSM-5 criteria specify that adequate opportunity for sleep must be present, and that dissatisfaction with sleep quality or quantity be present. Finally, the 
DSM-5 criteria include early morning awakenings, and no longer include nonrestorative sleep as diagnostic criteria.

While approximately $33 \%$ of adults report symptoms of insomnia, the prevalence of insomnia disorder according to DSM-5 diagnostic criteria in the general population ranges from 6\% to 10\% (American Psychiatric Association, 2013; Chung et al., 2015; Morgan, 2012). In a national Canadian sample (Morin, LeBlanc, et al., 2011), 40\% of respondents reported at least one symptom of insomnia, with $13 \%$ meeting criteria for insomnia disorder according to DSMIV-TR (American Psychiatric Association, 2000) diagnostic criteria. Women are approximately 1.4 times more likely than men to be diagnosed with insomnia, and this sex difference increases with age (Zhang \& Wing, 2006). Insomnia is more prevalent among middle age and older adults (Ohayon, 2002; Ohayon \& Reynolds, 2009), and specific sleep difficulties differ by age group, such that younger adults more commonly complain of sleep initiation difficulties whereas middle age and older adults commonly complain of sleep maintenance difficulties (Morin \& Jarrin, 2013).

The prevalence of DSM-5 insomnia increases to $10 \%$ to $20 \%$ in primary care settings, and $40 \%$ to $50 \%$ of individuals with insomnia have a co-occurring mental disorder (American Psychiatric Association, 2013). The prevalence of insomnia varies depending on the diagnostic criteria used. Chung et al. (2015) demonstrated that use of DSM-5 criteria results in a prevalence of $10.8 \%$ in a Chinese sample, which was approximately half the $22.1 \%$ prevalence of insomnia diagnosed using DSM-IV-TR criteria. Insomnia is a persistent disorder, and while persistence rates vary as a function of study design, there is evidence that over one-, three- and four-year periods, $74 \%$, $46 \%$ and $36 \%$ of people with insomnia, respectively, continue to exhibit insomnia symptoms (Morgan \& Clarke, 1997; Morin et al., 2009). 
There is evidence that individuals with a medical disorder are over twice as likely to be diagnosed with insomnia relative to those without a medical disorder, with disorders such as stomach ulcers, neurological problems, chronic obstructive pulmonary disease (COPD), migraines, and arthritis being associated with the greatest odds of developing insomnia (Budhiraja, Roth, Hudgel, Budhiraja, \& Drake, 2011). Furthermore, the prevalence of insomnia increases with each additional co-occurring medical disorder. Budhiraja and colleagues reported that while the prevalence of insomnia using DSM-IV diagnostic criteria among individuals without any medical disorder was $14.8 \%$, the prevalence increased to $21.9 \%$ among those with one medical disorder, $28.3 \%$ among those with two disorders, $33.8 \%$ among those with three disorders, and 38.9\% among those with more than three disorders. There are several potential explanations for the higher prevalence of insomnia among individuals with medical disorders. In addition to the possibility that specific medical disorders play a direct causal role in insomnia, is possible that they are indirectly associated with insomnia via pain and/or discomfort, side effects from pharmacological or surgical treatment, or physiological inflammation accompanying certain disorders (Budhiraja et al., 2011). Insomnia has been associated with immune functioning, as described below, and may therefore have a bidirectional association with medical disorders, potentially playing an etiological role or exacerbating preexisting medical conditions.

\section{Insomnia and Immune Functioning}

The two main branches of the immune system — adaptive and innate immunity—are impacted by sleep disturbance and circadian system. Adaptive immunity refers to the response of white blood cells, or lymphocytes, which search and destroy microbes based on immunological memory of having responded to similar threats in the past (Irwin, 2015). Impaired adaptive immunity results either in immune deficiency, in which the body's ability to combat infection is 
compromised, or in an autoimmune response, in which the immune response is exaggerated and causes damage to the body. Innate immunity refers to the rapid inflammation response that occurs when immune cells (e.g., monocytes, macrophages, and dendritic cells) detect any type of pathogen. This inflammation response contains the threat and promotes healing. Sleep disturbance has been associated with reduced adaptive immune response, or immune deficiency, and increased innate immune response, or cellular-level inflammation (Irwin).

Both the circadian system and sleep disturbance are associated with various aspects of immune functioning. Circadian rhythm is associated, via cortisol levels, with the number of immune cells such as monocytes and lymphocytes (e.g., CD4+ and CD8+ T cells) circulating throughout the body, with cell numbers reaching a maximum in the late evening and a minimum in the early morning (Born, Lange, Hansen, Molle, \& Fehm, 1997). Although the number of immune cells in the body is generally dependent on circadian factors, other aspects of immune functioning are dependent on nocturnal sleep. Natural killer (NK) lymphocyte numbers and activity are lowest during the first few hours of sleep, and increase during the late morning (Kronfol, Nair, Zhang, Hill, \& Brown 1997). Partial night sleep deprivation for a single night is associated with decreased NK cell numbers and activity, with a night of recovery sleep resulting in rebounded NK functioning (Irwin et al., 1996). Similarly, self-reported insomnia has been associated with decreased NK cell activity (Taylor, Lichstein, \& Durrence, 2003), and relative to healthy controls, decreased NK activity has been observed among individuals with insomnia (Irwin, Clark, Kennedy, Gillin, \& Ziegler, 2003). Savard, Laroche, Simard, Ivers, and Morin (2003) compared several immune measures between groups of good versus poor sleepers at two time points and reported that poor sleepers had lower levels of CD3+, CD4+, and CD8+ cells and that these levels decreased over a one week period among poor sleepers. 
Not only is sleep disturbance associated with immune functioning at the cellular level, but there is also evidence that it is associated with increased risk of contracting infectious diseases such as pneumonia (Patel et al., 2012) and the common cold (Cohen, Doyle, Alper, Janicki-Deverts, \& Turner, 2009). In a prospective longitudinal study, relative to eight-hour sleepers, women who slept less than five hours, or more than nine hours per night were 1.4 times more likely to have contracted pneumonia, and those who perceived they had insufficient sleep were 1.5 times more likely to have contracted pneumonia, after adjusting for age, comorbid conditions, and alcohol, caffeine, and tobacco use (Patel et al., 2012). Cohen and colleagues (2009) monitored sleep duration and efficiency for a two-week period before quarantining and exposing participants to a rhinovirus, and reported that those reporting less than seven hours of sleep per night were 2.9 times more likely to develop a cold than those reporting eight or more hours of sleep. Similarly, sleep efficiency, which is the percent of time in bed spent sleeping versus awake, was associated with greater likelihood of developing a cold, with those reporting a sleep efficiency of $92 \%$ or less being 5.5 times more likely to develop a cold than those reporting a sleep efficiency of $98 \%$ or more (Cohen et al., 2009).

\section{Human Immunodeficiency Virus}

Given the association between sleep disturbance, impaired immune functioning, and susceptibility to infectious disease, insomnia may be of particular relevance for autoimmune diseases such as human immunodeficiency virus (HIV). HIV is a viral infection that weakens the immune system by infecting and destroying CD4+ T cells, which are the cells that orchestrate immune response. By destroying CD4+ T cells, HIV leaves individuals susceptible to opportunistic infections (e.g., pneumonia), neurological impairment (e.g., dementia), and malignancies (e.g., lymphoma, Kaposi’s sarcoma; Public Health Agency of Canada, 2016). 
Course of HIV infection. Acute HIV infection refers to the period immediately after initial infection when seroconversion occurs over a period of several weeks to months. During acute HIV infection, approximately $90 \%$ of individuals experience fever, and $40 \%-80 \%$ of individuals experience other mild, non-specific symptoms such as muscle pain, sore throat, fatigue, headache, swollen lymph nodes, and/or skin rash. Up to $40 \%$ of individuals may experience nausea, vomiting, diarrhea, oral and/or genital ulcers, and/or weight loss. These symptoms appear two to four weeks after initial infection and may last anywhere from one to two weeks to several months. Approximately $10 \%$ of individuals remain asymptomatic during acute HIV infection (Public Health Agency of Canada, 2016). During acute HIV infection, viral levels are higher than during later stages as the immune system is only starting to produce antibodies, and CD4 count $\left(\mathrm{CD} 4+\mathrm{T}\right.$ cells $/ \mathrm{mm}^{3}$, an important measure of adaptive immune response) are reduced. Viral load reaches a peak between approximately 20 and 54 days after initial infection, and it is during this window that risk of transmission is greatest (Pilcher et al., 2004).

After acute HIV infection, individuals enter the stage of chronic asymptomatic infection characterized by continued viral replication at low levels and an adequate immune response as measured by a CD4 count $>200$ cells $/ \mu L^{3}$. In healthy individuals, CD4 count ranges from 500 to 1,600 cells $/ \mu \mathrm{L}^{3}$. If untreated, individuals remain asymptomatic for an average of ten years before progressing to symptomatic HIV infection and Acquired Immune Deficiency Syndrome (AIDS; Public Health Agency of Canada, 2016).

Symptomatic HIV infection is characterized by a CD4 count $<200$ cells $/ \mu \mathrm{L}^{3}$ and accompanying symptoms including, but not limited to: weight loss $>10 \%$ of body weight, chronic diarrhea (greater than three weeks), fatigue, dry cough with breathlessness, loss of 
vision, and chronic or recurrent Candida infections of the esophagus, mouth, or other mucosal surfaces (Public Health Agency of Canada, 2016). If left untreated, life expectancy after progressing to symptomatic HIV infection is approximately three years unless an opportunistic illness is present, in which case life expectancy is one year. Opportunistic infections include, but are not limited to: bacterial pneumonia, Kaposi's sarcoma, lymphoma and other types of cancer, herpes simplex virus, salmonella septicemia, candidiasis, toxoplasmosis, wasting syndrome, and a range of other infections. AIDS is diagnosed among HIV positive individuals when one or more opportunistic infections are present regardless of CD4 count (Public Health Agency of Canada). The World Health Organization has established an immunological classification for severity of HIV infection based on CD4 values, with values $>500$ representing no or not significant immunodeficiency, 350-499 representing mild immunodeficiency, 200-349 representing advanced immunodeficiency, and $<200$ cells $/ \mathrm{mm}^{3}$ representing severe immunodeficiency (World Health Organization, 2007).

HIV epidemiology. Since the beginning of the global HIV pandemic, over 35 million people have died and approximately 37 million are living with the virus worldwide (World Health Organization, 2018). In Canada, approximately 75,500 people were estimated to be living with HIV in 2014 (Public Health Agency of Canada, 2015). Of these, approximately 22\% were female, $49 \%$ contracted HIV via sex between men (e.g., men who have sex with men exposure category), and $21 \%$ were unaware of their HIV diagnosis. Although the incidence rate of HIV infection in Canada has declined by $14.5 \%$ since 2008, there were an estimated 2,570 new infections in 2014 (23.2\% female). The most frequently reported exposure category for HIV transmission was men who have sex with men, which accounted for $54.3 \%$ of all new HIV infections in 2014, followed by heterosexual contact and intravenous drug use, which accounted 
for $32.6 \%$ and $10.5 \%$ of new infections, respectively (Public Health Agency of Canada, 2015).

Despite the decline in HIV incidence in Canada since 2008, HIV is increasing in prevalence, with approximately 75,500 Canadians currently living with HIV infection, a 9.7\% prevalence increase since 2011 .

Combination antiretroviral therapy. Although there is no cure for HIV, combination antiretroviral therapy (cART) is the gold standard for treating infection and managing symptoms. In addition to improving immune functioning and decreasing HIV replication, cART has been shown to increase life expectancy to rates comparable to non-infected individuals (Samji et al., 2013). In order for cART to effectively suppress HIV replication, an adherence rate of $95 \%$ must be achieved (Chesney, 2003); otherwise, the individual is at risk for complications such as virologic failure, medication resistance and viral mutations (Battaglioli-DeNero, 2007). Individuals with low cART adherence have a five times greater risk of disease progression to AIDS than those with moderate or high cART adherence (Kitahata et al., 2004).

Maintaining a high level of adherence to cART is therefore imperative for reducing viral load and increasing quality of life and life expectancy. A number of psychosocial factors have been associated with reduced cART adherence, including untreated depression (Horberg et al., 2008; Uthman, Magidson, Safren, \& Nachega, 2014) and insomnia (Gay et al., 2011; Phillips et al., 2005; Saberi, Neilands, \& Johnson, 2011). Saberi et al. (2011) reported a linear relation between sleep quality and medication adherence such that poorer sleep quality was associated with greater non-adherence. Gay et al. (2011) reported that trouble sleeping and difficulty concentrating were associated with a greater likelihood of medication non-adherence. Phillips et al. (2005) reported that HIV-positive women who were poor sleepers reported poorer medication adherence relative to good sleepers. Poor sleepers reported missing medication due to 
forgetfulness, wanting to avoid side effects, not wanting to be noticed by others, experiencing changes in daily routine, falling asleep at dose time, and running out of pills. These studies demonstrate the association between poor sleep and cART adherence. Given the importance of cART adherence in successfully managing HIV infection and preventing AIDS, sleep may play an important role in the successful management of HIV, not only indirectly via cART adherence, but also more directly via immune functioning.

\section{Insomnia and HIV}

Prevalence of insomnia among PWH. It is difficult to estimate the exact prevalence of insomnia among people living with HIV (PWH), given the wide variety of self-report questionnaires used to measure sleep quality and disturbance in epidemiological research. One review reported a prevalence of insomnia assessed via self-report surveys ranging from $33 \%$ to 100\% (Smith, Huang, \& Manber, 2005). Another review noted a similar range in prevalence depending on the measure used and operationalization of sleep disturbance (Reid \& Dwyer, 2005). One study that used the Pittsburgh Sleep Quality Index (PSQI; Buysse, Reynolds, Monk, Berman, \& Kupfer, 1989), an empirically validated and widely used measure of sleep quality, reported a prevalence of insomnia of 73\% among PWH (Rubinstein \& Selwyn, 1998). This prevalence of $73 \%$ is widely cited in literature examining insomnia among PWH (e.g., Cruess et al., 2003; Robbins, Phillips, Dudgeon, \& Hand, 2004; Saberi et al., 2011). This is in stark contrast to the much lower prevalence of insomnia in the general population, which is estimated at $6 \%$ to $10 \%$ using DSM-5 criteria (American Psychiatric Association, 2013), or 13\% using DSM-IV-TR criteria (Morin, LeBlanc, et al., 2011). It is important to note here that most studies reporting on the prevalence of insomnia among PWH have not assessed insomnia using diagnostic criteria, but have instead used self-report questionnaires such as the PSQI that are not 
intended to be used for diagnostic purposes, therefore the exact prevalence of insomnia among PWH is unknown (Low, Goforth, Preud'homme, Edinger, \& Krystal, 2014). In the absence of prevalence estimates based on diagnostic criteria, estimates based on self-report have been used in the literature.

The prevalence of insomnia among PWH may be elevated relative to those living with multiple, co-occurring medical disorders; however, differences in research methodologies complicate comparison of rates between studies. As mentioned above, the prevalence of DSMIV-TR insomnia reported by Budhiraja et al. (2011) was $21.9 \%$ among those with one medical disorder, $28.3 \%$ among those with two disorders, $33.8 \%$ among those with three disorders, and $38.9 \%$ among those with over three disorders, whereas the prevalence of insomnia based on selfreport has been estimated at around 73\% (Rubinstein \& Selwyn, 1998). Even accounting for the multiple comorbidities experienced by $\mathrm{PWH}$, a prevalence rate of $73 \%$ is almost twice the rate observed in people with over three medical disorders in the general population; however, this estimate is not based on DSM-IV diagnostic criteria. More research is needed to determine more precise estimates of insomnia prevalence among $\mathrm{PWH}$, and to facilitate comparisons between studies.

Specific forms of sleep disturbance among PWH. Despite the lack of consensus on exact prevalence rates, the literature is replete with evidence of sleep disturbance among PWH (Reid \& Dwyer, 2005; Smith, Huang, \& Manber, 2005). Such disturbances include self-report as well as polysomnographic evidence. Robbins et al. (2004) reported that 33\% of PWH in their study reported a sleep onset latency, which is the length of time it takes to fall asleep, of over 60 minutes at least three times per week, $57 \%$ reported sleep efficiency $<85 \%$, and $90 \%$ reported $\geq$ 10 sleep disturbances throughout the night. Lee et al. (2012) reported that in a sample of 290 
PWH, 34\% reported sleep onset latency $>30$ minutes, and 56\% reported problems maintaining sleep (wake after sleep onset of 15\%). Gamaldo et al. (2013) reported that relative to HIVnegative controls, PWH had significantly elevated scores on the PSQI (8.8 vs. 5.3), the Insomnia Severity Index (ISI; Morin, 1993), a measure of insomnia symptom severity (13.3 vs. 4.3), and the Fatigue Severity Scale (FSS; Krupp, LaRocca, Muir-Nash, \& Steinberg, 1989), a measure of fatigue severity among adults with chronic illness (33.9 vs. 22.7). Similarly, Wibbeler, Reichelt, Husstedt, and Evers (2012) reported that relative to age- and sex-matched HIV-negative controls, PWH had significantly elevated scores on the PSQI (8.0 vs. 4.1) and the Epworth Sleepiness Scale (ESS; Johns, 1991), a measure of daytime sleepiness (9.4 vs. 6.5).

Abnormal sleep architecture has been observed among PWH, although not all studies have reported consistent results. While slow wave sleep typically occurs more frequently during earlier sleep cycles, several polysomnographic studies have demonstrated an overall increase in slow wave sleep, and more specifically, in later sleep cycles among PWH (Norman et al., 1992; White et al., 1995), however, other studies have not supported these findings (Ferini-Strambi et al., 1995; Wiegand et al., 1991). Relative to HIV-negative controls, Ferini-Strambi et al. observed an overall decrease in slow wave sleep and an increase in the rate of cyclic alternating pattern, a measure of unstable sleep (Parrino, Ferri, Bruni, \& Terzano, 2012), among PWH who had no subjective sleep complaints. Wiegand et al. observed normal percentages of slow wave sleep and rapid eye movement (REM) sleep in a small sample of men living with HIV, but noted longer sleep onset latency and total wake time, and reduced total sleep time and sleep efficiency. In a comparison of age-, sex-, and psychiatric diagnosis-matched insomnia patients with and without HIV, Low et al. (2012) observed significantly longer sleep onset latency (23 vs. 13 minutes), decreased sleep efficiency ( $74 \%$ vs. $82 \%$ ), and a decrease in time spent in REM sleep 
(13\% vs. $23 \%$ ) among the HIV-positive group. Other studies have demonstrated an increase in REM sleep during earlier sleep cycles relative to HIV-negative controls (Norman et al., 1992). Inconsistent findings across studies may result from different sample characteristics, study designs, methodologies, or covariates not included in the analyses. To further complicate comparisons between studies, pharmacological treatment of HIV has changed greatly since the beginning of the HIV pandemic, and treatment regime may confound comparisons between studies. Despite inconsistencies in the literature, sleep disturbance among PWH has been well documented via polysomnographic and self-report measures.

Insomnia and immune functioning among PWH. Among PWH, there is evidence for an association between insomnia and immune functioning; however, this evidence is mixed, possibly due to methodological differences in measuring insomnia. Several studies have reported that insomnia is associated with lower CD4 count (Brown, Mitler, \& Atkinson, 1991; Darko, McCutchan, Kripke, Gillin, \& Golshan 1992); however these were conducted prior to the widespread use of cART. A more recent study reported that sleep disturbance, as measured using the sleep disturbance subscale of the PSQI, was associated with lower CD4 count among women living with HIV, but that poor subjective sleep quality, as measured using overall PSQI score, was not (Seay et al., 2013). Other studies have found no association between insomnia and CD4 count (Cohen, Ferrans, Vizgirda, Kunkle, \& Cloninger, 1996; Nokes \& Kendrew, 2001; Robbins et al., 2004). It is possible that CD4 count is associated with specific sleep parameters rather than insomnia overall. For example, Lee, Portillo, and Miramontes (2001) reported that lower CD4 count was associated with number of awakenings, but not sleep efficiency, and Lee et al. (2012) reported that lower CD4 count was associated with greater sleep fragmentation. Although insomnia is not universally associated with CD4 count, some studies have reported an 
association between insomnia and other indicators of immune functioning such as CD3CD8 count (Cruess et al., 2003), which represent cytotoxic T cells, which destroy damaged and virusinfected cells, rather than helper T cells, which assist in producing antibodies (Irwin, 2015). Cruess et al. reported that poor subjective sleep quality mediated the relation between psychological distress and CD3CD8 count among PWH. Cytokines are proteins produced by immune cells used in the inflammation response, and specific interleukin cytokines have been associated with self-reported sleep initiation difficulty among people living with HIV, suggesting a link between immune-related inflammation and difficulty falling asleep (Gay, Zak, Lerdal, Pullinger, Aouizerat, \& Lee, 2015).

Similar equivocal findings have been reported regarding the association between insomnia and stage of HIV illness. Research has shown that insomnia is not associated with HIV progression up until the point of late-stage illness during which an AIDS-defining illness is present (Moeller et al., 1991; Reid \& Dwyer, 2005). Moeller et al. reported that total PSQI score as well as sleep onset latency were higher among individuals with symptomatic HIV infection than in other stages of the illness. There is no evidence that HIV severity played a causal role, and it is possible that insomnia symptoms were the direct result of an AIDS-defining illness, psychiatric comorbidity, or other variables. Rubinstein and Selwyn (1998) observed that HIV severity was not associated with sleep disturbance, and that cognitive impairment and depression were the strongest predictors of insomnia in multivariate analyses. Saberi et al. (2011) reported that HIV viral load was independently associated with poor quality of sleep. Conversely, Robbins et al. (2004) reported that viral load and HIV-related physiological symptoms were not associated with quality of sleep. In sum, there is mixed evidence for an association between 
insomnia and both immune functioning and HIV severity. It is possible that CD4 count and viral load are associated with specific indicators of poor sleep rather than insomnia in general.

Etiology of insomnia among PWH. It remains unclear why the prevalence of insomnia is so disproportionately high among PWH. Cognitive, physiological, and psychosocial factors have all been proposed as possible factors in the onset and maintenance of insomnia among PWH (Taibi, 2013). Cognitive factors include worrying and unhelpful beliefs about sleep, which are associated with insomnia in the general population (Morin \& Benca, 2012). Physiological factors include the direct impact of the virus on sleep parameters such as slow wave and REM sleep (Norman et al., 1992), symptoms of comorbid medical conditions, and antiretroviral medication side effects. One particular antiretroviral medication used in the treatment of HIV, efavirenz, has been associated with vivid dreams and insomnia (Kenedi \& Goforth, 2011), and more specifically, increased sleep onset latency and decreased sleep efficiency relative to nonefavirenz antiretroviral treatment (Gallego et al., 2004). Efavirenz has been associated with an increase in insomnia among $4 \%$ to $16 \%$ of individuals taking the drug, and is not universally prescribed; therefore, it alone cannot explain the high prevalence of insomnia among PWH (Kenedi \& Goforth, 2011). Efavirenz has also been associated with elevated anxiety among $16 \%-35 \%$ of individuals, and depression among $8 \%$ of individuals, with severe depression reported among half of those affected by depression symptoms (Kenedi \& Goforth, 2011). Psychosocial factors include the stress of living with a stigmatized and chronic medical condition, financial problems, and comorbid mental health problems such as depression and anxiety (Cruess et al., 2003).

Although evidence exists for an association between insomnia and many of the cognitive, physiological, and psychosocial factors above, much of the evidence is equivocal and the 
etiology of insomnia among PWH remains unknown. Several studies have examined the impact of above variables on insomnia, including HIV progression, immune functioning, and psychosocial factors, and multivariate analyses have consistently highlighted depression as the most important predictor, independent of cART treatment type, duration of HIV infection, and immunological variables (Crum-Cianflone et al., 2012; Jean-Louis et al., 2012; Wibbeler et al., 2012).

Comorbid depression and insomnia among PWH. Depression, which is a common comorbid condition observed in people with insomnia as well as those living with HIV, appears to be an important variable in understanding and treating insomnia among this population. It is estimated that 10\%-20\% of adults with insomnia also suffer from depression (Ohayon, 2007), and similarly, $10 \%-20 \%$ of PWH are also depressed (Klinkenberg \& Sacks, 2004). Women living with HIV experience higher rates of depression than their male counterparts (Evans et al., 2002). In terms of lifetime prevalence, $30 \%-50 \%$ of PWH will experience major depression at some point (Klinkenberg \& Sacks, 2004). Wibbeler et al. (2012) reported a higher rate of depression among PWH relative to sex- and age-matched HIV-negative controls, with 63.5\% vs. $12.6 \%$ of participants reporting a score greater than eight on a German version of the Beck Depression Inventory-Second Edition (BDI-II; Beck, Steer, \& Brown, 1996), a measure of depressive symptom severity, on which a score of eight is indicative of mild depressive symptoms (Hautzinger, Keller, \& Kühner, 2009). Crum-Cianflone et al. (2012) reported that the prevalence of insomnia among PWH without depression, and with mild, moderate, and severe depression was $37 \%, 71 \%, 89 \%$, and $93 \%$, respectively, further illustrating the association between insomnia and depression among PWH. 
There is evidence that the directional nature of the relation between depression and insomnia is bidirectional (Sivertsen et al., 2012), yet in most cases, insomnia is observed prior to the onset of depression (Ohayon \& Roth, 2003). The directional nature of the relation between depression and HIV infection is less clear. It is possible that depression could serve as a risk factor for engaging in behaviours considered high risk for contracting HIV such as unprotected sexual intercourse or intravenous drug use. Alternatively, depression could result from HIVrelated events such as dealing with HIV stigma, medication side-effects, experiencing opportunistic infections and other physical health problems (Goforth, Cohen, \& Murrough, 2008), or HIV may damage subcortical brain structures resulting in feelings of hopelessness and demoralization (Treisman \& Angelino, 2007). Regardless of the directional nature of the relation between depression and HIV infection, the presence of depressed mood has been associated with more rapid disease progression as measured by CD4 count and viral load (Ironson et al., 2005). In addition, severity of depressive symptoms has been associated with increased mortality among HIV positive women (Ickovics et al., 2001). As mentioned above, untreated depression has been associated with reduced cART adherence (Horberg et al., 2008). Major depression, therefore, is not only highly prevalent among individuals living with HIV, but it has been associated with decreased cART adherence, more rapid disease progression, and increased mortality.

Among PWH who report insomnia, depressive symptoms have been shown to mediate the association between poor sleep quality and cART non-adherence (Phillips et al., 2005; Saberi et al., 2011). Given the elevated rates of both insomnia and depression among PWH (Klinkenberg \& Sacks, 2004; Rubinstein \& Selwyn, 1998), and given the association between sleep disturbance, impaired immune functioning, and susceptibility to infectious disease, CBT-I (Edinger \& Carney, 2008) may provide important health benefits for this population. Such 
benefits may include improved sleep, reduced depressive symptomology, improved cART adherence, and corresponding immunological and physical health benefits.

\section{Treatment of Insomnia}

CBT-I. CBT-I (Edinger \& Carney, 2008; Edinger, Hoelscher, Marsh, Lipper, \& Ionescue-Pioggia, 1992; Hoelscher \& Edinger, 1988; Morin, 1993; Morin, Kowatch, Barry, \& Walton, 1993) is the gold standard treatment for insomnia as recommended by the American Academy of Sleep Medicine (Schutte-Rodin, Broch, Buysse, Dorsey, \& Sateia, 2008) and the American College of Physicians (Qaseem, Kansagara, Forciea, Cooke, \& Denberg, 2016). It is a 4-session cognitive behavioural therapy for insomnia administered biweekly in individual format, and involves sleep restriction, stimulus control, cognitive restructuring, and psychoeducation. More specifically, the first session involves presenting the treatment rationale and sleep psychoeducation, introducing a behavioural treatment regimen consisting of a series of sleep habit parameters to follow, and determining a personalized "time in bed" prescription. The time in bed prescription is the mutually agreed upon window of time during each 24-hour period designated as the only time an individual should be in bed. The second session involves discussing the role of cognitions in insomnia, followed by cognitive restructuring and presenting constructive worrying techniques. The third and fourth sessions are used as follow-up sessions to assist the participant in adjusting "time in bed" prescriptions, to positively reinforce their efforts and success, and to help problem-solve any problems they might have encountered.

Efficacy of CBT-I. There is a wealth of evidence supporting the efficacy of CBT-I. In a comparison of CBT-I with relaxation therapy and a placebo control therapy, CBT-I resulted in greater improvements in sleep efficiency and sleep fragmentation, and was the only treatment condition that resulted in improved total sleep time (Edinger, Wohlgemuth, Radtke, Marsh, \& 
Quillian, 2001). In a randomized controlled trial pilot study, Manber et al. (2008) reported that CBT-I in combination with the antidepressant medication escitalopram resulted in higher rates of remission of both insomnia and depression, relative to escitalopram combined with a placebo control condition. Furthermore, in a randomized, double-blinded, placebo-controlled trial, CBT-I was compared with the sleep medication zopiclone and a placebo medication (Sivertsen et al., 2006). Sivertsen et al. (2006) reported that only CBT-I resulted in improved sleep efficiency, and that participants receiving CBT-I evidenced greater time spent in slow wave sleep and lower total wake times relative to the other groups six months posttreatment. This study concluded that CBT-I is superior to the sleep medication zopiclone in treating insomnia, and demonstrated the durability of CBT-I up to six months posttreatment. Other studies have also demonstrated that CBT-I is superior (Jacobs, Pace-Schott, Stickgold, \& Otto, 2004) or equivalent (Morin, Colecchi, Stone, Sood, \& Brink, 1999) to pharmacotherapy for insomnia. An early systematic review demonstrated that CBT-I was superior to any standalone treatments such as stimulus control, sleep restriction, psychoeducation, relaxation training, sleep hygiene, and pharmacological treatment (Wang, Wang, \& Tsai, 2005).

Mediators of CBT-I outcomes. Cognitive, behavioural, and hyperarousal models of insomnia propose several mediators of treatment outcome, including decreased maladaptive beliefs and attitudes about sleep, decreased sleep effort, increased sleep-related self-efficacy, decreased physiological and cognitive arousal, decreased napping, decreased time in bed, and decreased bedtime and rise time variability (Schwartz \& Carney, 2012). A meta-analytic review of the effectiveness of CBT-I reported medium to large effect sizes for short-term and long-term sleep variables (Okajima, Komada, \& Inoue, 2011). There is evidence that CBT-I is effective at treating insomnia among individuals with a wide range of comorbid psychiatric disorders (Taylor 
\& Pruiksma, 2014) such as depression (Lancee, van den Bout, van Straten, \& Spoormaker, 2013; Manber et al., 2008) and post-traumatic stress disorder (Germain, Shear, Hall, \& Buysse, 2007), as well as comorbid medical disorders such as medium to severe chronic pain (Currie, Wilson, \& Curran, 2002; Jungquist et al., 2010; 2012), fibromyalgia (Martínez et al., 2014), and cancer (Garland et al., 2014).

CBT-I among PWH. Surprisingly, no study to date has examined the efficacy of CBT-I among PWH. People living with HIV have frequently been excluded from randomized controlled CBT-I trials, given the complex association between sleep and HIV infection, and that a common exclusion criteria for CBT-I trials is having a medical condition that compromises sleep (Edinger et al., 2001; Edinger, Wohlmeguth, Radtke, Coffman, \& Carney, 2007; Morin et al., 1999; Savard et al., 2003; Sunnhed \& Jansson-Fröjmark, 2015). Only three interventions for insomnia among PWH have been reported (Taibi, 2013), with none using CBT-I, the first-line treatment as recommended by American Academy of Sleep Medicine (Schutte-Rodin et al., 2008). Using a pretest/posttest design, Phillips and Skelton (2001) administered acupuncture in a group format twice weekly for five weeks to $23 \mathrm{HIV}$-positive adults with sleep disturbance. Although posttest scores were not reported, there was a statistically significant change in total sleep time, total wake time, sleep percentage, wake percentage, sleep ratio, and subjective sleep quality. There was no change in sleep onset latency, number of awakenings, and length of awakenings. In an internet-administered study among PWH with sleep disturbance, Dreher (2003) examined whether reducing caffeine intake by $90 \%$ for 30 days improved sleep relative to individuals who continued their usual caffeine intake. Dreher reported statistically significant improvements in sleep quality and well-being in the experimental, but not control group, and that at posttreatment, relative to the control group, individuals in the experimental group had better 
sleep quality as indicated by lower scores on the PSQI (7.4 vs. 10.2). Finally, Hudson, Portillo, \& Lee (2008) asked 30 women living with HIV to implement over the course of a week two of six possible sleep hygiene techniques from the Sleep B.E.T.T.E.R. exercise (an acronym for remembering sleep hygiene techniques related to the bedroom, exercise, tension, time to sleep, eating, drinking and drugs, and rhythm). Hudson et al. reported that although there was no overall effect on sleep onset latency, there was a statistically significant reduction in sleep onset latency only among those participants $(n=6)$ who took $>30$ minutes to fall asleep at baseline. Similarly, the intervention had no overall effect on total sleep time or number of awakenings, but the authors reported improved sleep efficiency only among those participants $(n=7)$ whose sleep efficiency was $<70 \%$ at baseline.

Given the paucity of research examining sleep interventions among PWH, there are currently no standards of practice for treating insomnia with this population (Taibi, 2013). As noted above, insomnia is present in almost three quarters of PWH (Rubinstein \& Selwyn, 1998) and is associated with poorer immune functioning (Patel et al., 2012), and cART non-adherence, which is essential for managing HIV (Saberi et al., 2011). Empirically-validated studies demonstrating the efficacy of CBT-I, the first-line treatment for insomnia, among PWH are therefore strongly warranted.

\section{Patient Perspectives on Insomnia}

In order to gain a deeper understanding of the patient experience for individuals living with insomnia and undergoing treatment, researchers have employed qualitative research methods such as interviews and focus groups (Carey, Moul, Pilkonis, Germain, \& Buysse, 2005; Cheung, Bartlett, Armour, Glozier, \& Saini, 2014; Green, Hicks, \& Wilson, 2008; Harvey et al., 2013; Henry, Rosenthal, Dedrick, \& Taylor, 2013; Kyle, Espie, \& Morgan, 2010; Kyle, Morgan, 
Spiegelhalder, \& Espie, 2011). Insomnia patients commonly reported that general practitioners did not understand their experiences with insomnia nor did they provide satisfactory information about insomnia and its treatment (Carey et al., 2005; Green et al., 2008). Regarding perceived causes of insomnia, common themes included major life events (Cheung et al., 2014), emotions, thinking patterns, and sleep-related emotions and thoughts (Harvey et al., 2013). Patients commonly noted the impact of insomnia on daytime functioning, specifically their ability to participate in social activities, safety while driving or working, decreased work productivity, and impaired interpersonal relationships, particularly with a primary partner when sleep problems interfered with physical and emotional intimacy (Cheung et al., 2014; Green et al., 2008; Henry et al., 2013; Kyle et al., 2010). Nighttime impairment included anger, frustration, and worry (Green et al., 2008); however, themes describing daytime impairment were more common than their nighttime counterparts in the qualitative literature, suggesting that the impact of insomnia on daytime functioning is of great concern for insomnia patients.

Qualitative explorations of the insomnia experience among cancer patients (Davidson, Feldman-Stewart, Brennenstuhl, \& Ram, 2007; Fleming, Gillespie, \& Espie, 2010) may have particular relevance for PWH. The majority of cancer survivors in one study (Fleming et al., 2010) reported that insomnia developed following cancer diagnosis, and those with preexisting insomnia reported aggravated symptoms following cancer diagnosis. Furthermore, patients attributed their insomnia not only to the direct effects of cancer and its treatment, but also to the disruption that active cancer treatment had on daily routines leading to napping, lingering in bed, staying up late, and inconsistent bed and rise times when off of work. Perhaps surprisingly, the majority of patients reported that the impact of insomnia was worse than the impact of cancer treatment (Fleming et al., 2010). Despite this, patients living with cancer and insomnia 
commonly reported not wanting to discuss insomnia with their physicians because they believed that cancer was more important, that physicians did not want to be bothered, and they feared that physicians would suggest treating insomnia with medication, which patients generally preferred to avoid (Davidson et al., 2007). Other commonly reported themes regarding the impact of insomnia among cancer patients included negative mood, physical symptoms, impaired interpersonal relationships, poor sleep quality, and cognitive consequences such as impaired memory and concentration, and increased worry about sleep (Fleming et al., 2010).

Qualitative exploration of individuals' causal attributions of sleep problems, experiences living with insomnia, and efforts seeking and undergoing treatment play an important role in designing safe, effective, feasible, and acceptable treatments for insomnia. Qualitative research may play an even greater role in designing specialized insomnia treatments for specific populations, such as people living with HIV, who may have additional treatment concerns not present in the general population, such as compromised immune functioning, multiple medical problems, and increased stress due to living with a stigmatized illness. To date, no qualitative studies have been published examining the experiences of people living with both HIV and insomnia. Similarly, there is not yet any quantitative research examining the efficacy of CBT-I among PWH. Given the unique psychosocial, biological and pharmacological characteristics of this population, a specially tailored version of CBT-I may be warranted; however, to date, no research has been published to this end.

\section{Current Study}

Rationale. The current study employed both quantitative and qualitative research methods to 1) explore and describe the characteristics, comorbidity, and covariates of insomnia among PWH, 2) identify the presence of relevant CBT-I treatment targets, 3) examine the safety, 
feasibility, acceptability, and efficacy of CBT-I in this population, an important step before implementing a full randomized controlled trial design (Robey, 2004; Sidani, 2015), and 4) examine participant perspectives to explore whether CBT-I could be tailored to better meet the needs of PWH. While polysomnography and questionnaires have been used to examine sleep parameters among PWH (Low et al., 2012; Norman et al., 1992; Robbins et al., 2004), this is the first study to date to incorporate two-week prospective self-monitoring using the Consensus Sleep Diary (CSD; Carney et al., 2012) with this population. While polysomnography is considered the "gold standard" for assessing breathing- or moving-related sleep disorders, it is not indicated for evaluating chronic insomnia (Schutte-Rodin et al., 2008). Rather, American Academy of Sleep Medicine guidelines recommend two-week sleep self-monitoring using sleep diaries such as the CSD to evaluate insomnia both before, and during treatment (Schutte-Rodin et al.). CBT-I treatment targets to be explored in the current study include daytime napping, extended time in bed (e.g., getting into bed early; sleeping in), engaging in non-sleep activities in the bedroom, and experiencing worry, anxiety, and/or physical tension when attempting to sleep (Smith \& Perlis, 2006).

Design. Given that no studies to date have examined the impact of CBT-I on PWH, and it is unknown whether CBT-I will be an efficacious treatment for insomnia in this population, a randomized controlled trial design would have been premature, as would recruiting a large number of participants to power a randomized controlled trial. An alternative to between-groups design is single-case research design, which encompasses several more specific designs such as interrupted time-series, $\mathrm{ABAB}$, multiple baseline, and changing-criterion designs (Kazdin, 1982). The current study implemented an interrupted time-series design, described in the Method section below, to examine change in insomnia severity, sleep efficiency, and total wake time 
among participants receiving CBT-I. Furthermore, an exit interview was conducted to explore participants' perceptions of CBT-I and to elicit feedback to inform whether CBT-I and the research methods used could be tailored to better meet the needs of this vulnerable population.

Although the absence of a treatment control group limits the ability to demonstrate that improvements are due solely to the treatment, the efficacy of CBT-I in other populations is well documented (Edinger et al., 2001; Okajima et al., 2011; Sivertsen et al., 2006), and this study examines the extent to which CBT-I impacts sleep- and HIV-related variables among PWH. Furthermore, the single-case interrupted time-series design incorporates several features, described in the Method section below, that reduce threat to internal validity.

Epistemology. Inherent in the research process, yet rarely made explicit, is the philosophical worldview, or epistemology, held by researchers themselves (Creswell, 2014). Although worldviews play an important role in guiding research design, they are rarely identified a priori in mixed methods research (Evans, Coon, \& Ume, 2011). A worldview involves a set of beliefs or assumptions that guide research decisions such as the type of approach or methods chosen. Four main worldviews that have been associated with different research approaches and methodologies are postpositivist, constructivist, transformative, and pragmatic (Creswell, 2014). The pragmatic worldview is concerned with understanding problems and exploring solutions using whatever approach necessary than with adhering to a specific approach or methodology (Rossman \& Wilson, 1985). Some assumptions of the pragmatic worldview are that 1) researchers should have the freedom to choose whichever approach, methodology, technique, or procedures are best suited for addressing research questions, 2) reality exists externally beyond the mind as well as within the mind, but discussions of reality distract from the goal of research, 3) truth is not absolute, but is whatever is most useful at understanding a given problem, and 4) 
research occurs in social, historical, and political contexts, and may incorporate a social justice lens as needed (Creswell, 2014). The pragmatic worldview is commonly associated with mixed method research, and has been used in trials that have implemented quantitative and qualitative research methods to evaluate the effectiveness and acceptability of interventions promoting mental and physical health (Goldstein et al., 2015; Khalil, Callaghan, Carter, \& Morres, 2012; Prowse, Meadows, \& Enticott, 2015; Taylor et al., 2012; Thomas et al., 2010).

Several features of this study align closely with the pragmatic worldview, such as the solution-focused goal of examining the safety, acceptability, feasibility, and intervention effects of CBT-I in a specific population, the use of a mixed methods approach, the assumption that there exist both an external reality which can be measured and an internal reality with which individuals understand and interpret the world, and the acknowledgment that PWH are a vulnerable, marginalized population in need of treatment protocols specially tailored to address specific health needs. The philosophical worldview inherent in the current study is therefore a pragmatic one.

Community Consultation. HIV stigma is a concern for PWH that may lead to discrimination by friends, family, employers, and even health care providers (Shacham, Rosenberg, Onen, Donovan, \& Overton, 2015). The risk of stigma in research with vulnerable populations may be reduced by consulting a community advisory board (CAB) composed of members of the community being studied, which is common in the field of HIV/AIDS research, (e.g., Burkhalter et al., 2013; Fasula et al., 2013; Loue, 2012; Villarruel, Gal, Eakin, Wilkes, \& Herbst, 2010). Community-engaged research varies along a continuum ranging from research with the community, where community members are seen as partners who assist with minor decisions, to community-based participatory research, where community members are seen as 
equal partners to investigators who share full control of study decisions, including how results are used and disseminated (Hacker, 2013). Given the pragmatic lens of the current study, and the need for greater investigator control due to the nature of the study as a dissertation project, the primary researcher decided to engage community members to inform methodological decisions without fully engaging in community-based participatory research.

Hypotheses. It was hypothesized that from baseline to post-treatment, participants would experience 1) significant reduction in subjective insomnia symptom severity, as measured by the ISI, 2) improvement in sleep efficiency, as measured using the CSD, with a target sleep efficiency between $85 \%$ and $90 \%, 3$ ) reduction in total wake time, as measured by the CSD, 4) reduction in viral load for participants with a detectable viral load at baseline, measured via selfreport, 5) increase in CD4 count, measured via self-report, and 6) improved cART medication adherence, measured via two self-report adherence measures described below. It was also hypothesized that CBT-I would be a safe, feasible, and acceptable treatment for insomnia among PWH, and feedback on tailoring CBT-I to meet the specific needs of this population was expected.

\section{Method}

\section{Participants}

The study received approval by the Ryerson University Research Ethics Board. Adults with comorbid HIV and insomnia were recruited to participate in the study between September 2016 and April 2018. Electronic advertisements were placed on Facebook, Twitter, gay social networking apps such as Scruff and Grindr, the Ryerson University HIV Prevention Lab website, and through listservs and newsletters of HIV community-based organizations that agreed to participate in recruitment activities. Letters were sent to physicians at local medical clinics that 
specialize in HIV treatment and testing, informing them of the purpose of the study and requesting their assistance with recruiting individuals who may meet inclusion criteria. Printed advertisements in the form of posters and pamphlets were placed at participating medical clinics. Advertisements mentioned that participants 1) must be living with HIV, and 2) currently experience trouble falling asleep, staying asleep throughout the night, or getting back to sleep after waking up too early, at least three nights per week for at least three months.

Inclusion and exclusion criteria. Participants were included if they: 1) were 18 years of age or older, 2) could understand and communicate in English, 3) were capable of providing informed consent, 4) had clinically significant insomnia based on an ISI score of $\geq 10$, which is the recommended cutoff indicative of insomnia among community samples (Morin, Belleville, Bélanger, \& Ivers, 2011), 5) were living with HIV, and 6) were willing to provide HIV viral load and CD4 count from blood work within the past two months. Canada's national standard of care for people living with HIV is to have routine blood work collected every three months (Public Health Agency of Canada, 2016), so participants would not be required to seek additional testing outside of their standard schedule. Participants were excluded if they reported: 1) active suicidal ideation, 2) psychotic symptoms, 3) unmanaged bipolar disorder, 4) presence of a severe substance use disorder according to DSM-5 criteria, and 5) presence of any breathing-related sleep disorders (e.g., obstructive sleep apnea/hypopnea, central sleep apnea, and sleep-related hypoventilation), or circadian rhythm sleep-wake disorders.

More stringent inclusion and exclusion criteria were used to identify potential participants to receive CBT-I. The more stringent inclusion criterion was greater insomnia severity based on an ISI score of $\geq 15$ rather than 10 , which corresponds to moderate insomnia severity (Morin, Belleville, et al., 2011), and has been used as a cutoff for inclusion criteria in 
CBT-I randomized controlled trial research (e.g., Carney et al., 2017). While a cutoff of $\geq 10$ is often used in community samples, the more stringent cutoff of $\geq 15$ was used for this study given that it is the first to examine the effects of CBT-I among PWH. In addition to the exclusion criteria listed above, participants were excluded from receiving CBT-I (but still eligible for the main study) if they reported: 1) hypnotic dependence (e.g., dependent on sleep medication), 2) working shift work or frequent time zone travel over the course of the study, 3) contingent or inconsistent hypnotic use, or anticipated change in hypnotic medication dose over the course of the study, 4) receiving psychotherapy for insomnia or any other mental disorder over the course of the study, and 5) presence of a current AIDS-defining opportunistic infection and/or a CD4 count $<200$ cells $/ \mu \mathrm{L}^{3}$. Participants with a current AIDS-defining opportunistic infection were excluded because unstable illness may prevent individuals from fully engaging in, and/or be exacerbated by, CBT-I (Smith \& Perlis, 2006). Potential participants who reported using cannabis for medical purposes were included if their cannabis use was non-contingent on sleep difficulty (i.e., used consistently throughout the course of the study and not only on nights when sleep difficulty is present). Participants invited to receive CBT-I were not randomly selected from the larger sample. Rather, the first ten participants who met the more stringent inclusion criteria for the CBT-I pilot study, and who also demonstrated the presence of at least one CBT-I treatment target were invited to receive CBT-I. A sample size of ten was determined to be sufficient based on the use of a single-case research design, described below.

Participant characteristics. The final sample included 48 PWH between the ages of 26 and $70(M=46.9, S D=12.6)$. Participants predominantly identified as male $(93.8 \%)$, gay $(87.5 \%)$, and White $(66.7 \%)$. With regard to nativity and immigration status, the majority of participants reported being born in Canada (58.3\%) and having Canadian citizenship or 
permanent resident status $(91.7 \%)$. Foreign-born participants reported emigrating from 16 countries from North and South America, Europe, Africa, and Asia, thus mirroring the multicultural population of Toronto. Most participants reported having a postsecondary degree or equivalent (58.3\%), and nine participants (18.8\%) reported currently being a student (either full or part time). With regard to employment status, just under half of participants (45.8\%) reported being employed either full- or part-time, including self-employment, and 21 participants (43.8\%) reported being unemployed or receiving long-term disability. The mean number of years since HIV diagnosis was $12.5(S D=9.3)$. Complete sociodemographic information is presented in Table 1.

Pilot study subsample. The subsample of ten participants in the CBT-I pilot study ranged in age from 26 to $62(M=48.0, S D=12.4)$. All identified as gay men, and the majority identified as White (80.0\%). All participants reported having Canadian citizenship or permanent resident status, and six reported being born in Canada. The foreign-born participants $(40.0 \%)$ reported emigrating from four different countries from North and South America, Europe, and Asia. Most participants reported obtaining a postsecondary degree or equivalent $(70.0 \%)$, and the remaining participants reported completing some postsecondary education. Half (50.0\%) reported full-time or self-employment, with the remainder reporting being unemployed, retired, or receiving longterm disability. The mean number of years since HIV diagnosis was $8.4(S D=7.9)$.

Sociodemographic differences between full sample and pilot study subsample. Chisquare analysis was conducted to determine whether the ten CBT-I participants differed from the rest of the larger sample on any sociodemographic variables. There were no statistically significant differences in gender, $\chi^{2}(1)=.84, p=.36$, sexual orientation, $\chi^{2}(3)=1.51, p=.68$, education level $\chi^{2}(6)=3.56, p=.74$, annual income, $\chi^{2}(9)=3.55, p=.94$, or ethnicity $\chi^{2}(10)=$ 
Table 1

Demographic Variables in the Full Sample and the Pilot Study Subsample

\begin{tabular}{|c|c|c|c|c|}
\hline \multirow[b]{2}{*}{ Variable } & \multicolumn{2}{|c|}{$\begin{array}{l}\text { Full sample } \\
\qquad(N=48)\end{array}$} & \multicolumn{2}{|c|}{$\begin{array}{l}\text { Pilot study subsample } \\
\qquad(n=10)\end{array}$} \\
\hline & $\%(n)$ & $M(S D)$ & $\%(n)$ & $M(S D)$ \\
\hline Age & & $46.9(12.6)$ & & $48.0(12.4)$ \\
\hline \multicolumn{5}{|l|}{ Sex } \\
\hline Male & $95.8(46)$ & & $100.0(10)$ & \\
\hline Female & $4.2(2)$ & & - & \\
\hline \multicolumn{5}{|l|}{ Gender $^{\mathrm{a}}$} \\
\hline Male & $93.8(45)$ & & $100.0(10)$ & \\
\hline Female & $6.3(2)$ & & - & \\
\hline Transwoman & $2.1(1)$ & & - & \\
\hline Two-Spirit & $2.1(1)$ & & $10.0(1)$ & \\
\hline \multicolumn{5}{|l|}{ Sexual orientation ${ }^{\mathrm{a}}$} \\
\hline Gay/homosexual & $87.5(42)$ & & $100.0(10)$ & \\
\hline Queer & $6.3(3)$ & & $20.0(2)$ & \\
\hline Straight/heterosexual & $4.2(2)$ & & - & \\
\hline Bisexual & $4.2(2)$ & & - & \\
\hline Asexual & $2.1(1)$ & & - & \\
\hline Two-Spirit & $2.1(1)$ & & $10.0(1)$ & \\
\hline \multicolumn{5}{|l|}{ Relationship status } \\
\hline Single & $58.3(28)$ & & $60.0(6)$ & \\
\hline Casual partner & $22.9(11)$ & & $20.0(2)$ & \\
\hline Living with partner $(>1$ year) & $18.8(9)$ & & $20.0(2)$ & \\
\hline \multicolumn{5}{|l|}{ Ethnicity most identified with } \\
\hline White (British, French, Other) & $69.3(33)$ & & $80.0(8)$ & \\
\hline Latin American & $6.3(3)$ & & $10.0(1)$ & \\
\hline Middle Eastern & $6.3(3)$ & & - & \\
\hline Asian (East/South) & $6.3(3)$ & & $10.0(1)$ & \\
\hline African & $4.2(2)$ & & - & \\
\hline Other (African-/Indo-Caribbean; Mixed) & $8.4(4)$ & & - & \\
\hline \multicolumn{5}{|l|}{ Education level $^{\mathrm{b}}$} \\
\hline High school diploma or less & $10.5(5)$ & & - & \\
\hline Some postsecondary education & $29.2(14)$ & & $30.0(3)$ & \\
\hline Completed postsecondary education & $35.4(17)$ & & $50.0(5)$ & \\
\hline Some or completed graduate education & $23.0(11)$ & & $20.0(2)$ & \\
\hline
\end{tabular}




\begin{tabular}{|c|c|c|c|c|}
\hline \multirow[b]{2}{*}{ Variable } & \multicolumn{2}{|c|}{$\begin{array}{l}\text { Full sample } \\
\qquad(N=48)\end{array}$} & \multicolumn{2}{|c|}{$\begin{array}{l}\text { Pilot study subset } \\
\qquad(n=10)\end{array}$} \\
\hline & $\%(n)$ & $M(S D)$ & $\%(n)$ & $M(S D)$ \\
\hline \multicolumn{5}{|l|}{ Annual income $\mathrm{b}^{\mathrm{b}}$} \\
\hline Under $\$ 20 \mathrm{k}$ & $43.8(21)$ & & $50.0(5)$ & \\
\hline$\$ 20 \mathrm{k}-\$ 39 \mathrm{k}$ & $10.5(5)$ & & - & \\
\hline$\$ 40 \mathrm{k}-\$ 59 \mathrm{k}$ & $16.6(8)$ & & $20.0(2)$ & \\
\hline$\$ 60 \mathrm{k}-\$ 79 \mathrm{k}$ & $12.5(6)$ & & $10.0(1)$ & \\
\hline Over $\$ 80 \mathrm{k}$ & $10.5(5)$ & & $20.0(2)$ & \\
\hline \multicolumn{5}{|l|}{ Employment status ${ }^{\mathrm{a}}$} \\
\hline Full time employed & $22.9(11)$ & & $40.0(4)$ & \\
\hline Part time employed & $12.5(6)$ & & - & \\
\hline Self employed & $10.5(5)$ & & $10.0(1)$ & \\
\hline Unemployed & $18.8(9)$ & & $10.0(1)$ & \\
\hline Long term disability & $27.1(13)$ & & $30.0(3)$ & \\
\hline Retired & $16.7(8)$ & & $10.0(1)$ & \\
\hline Years since HIV diagnosis & & $12.5(9.3)$ & & $8.4(7.9)$ \\
\hline Years living with insomnia ${ }^{b}$ & & $8.2(8.6)$ & & $7.5(8.0)$ \\
\hline Insomnia predates HIV diagnosis & $29.2(14)$ & & $40.0(4)$ & \\
\hline HIV diagnosis predates insomnia & $54.2(26)$ & & $30.0(3)$ & \\
\hline HIV and insomnia coincide & $12.5(6)$ & & $30.0(3)$ & \\
\hline
\end{tabular}

Note. Participants in the pilot study subset $(n=10)$ are also included in the full sample $(N=48)$. ${ }^{a}$ Variable response frequency $>100 \%$ due to some participants selecting more than one applicable option. ${ }^{b}$ Variable response frequency $<100 \%$ due to missing responses. 
5.99, $p=.82$. A Mann-Whitney $\mathrm{U}$ test revealed no statistically significant differences in age, $U=$ 173.0, $p=.67$, or time since HIV diagnosis, $U=117.5, p=.10$ between the CBT-I and nonCBT-I samples.

\section{Measures}

Screening measures. Participant eligibility was assessed using the following measures.

Insomnia Severity Index. The Insomnia Severity Index (ISI; Morin, 1993) was used to screen for insomnia. The ISI is a 7-item self-report scale that takes five minutes to administer (Savard, Savard, \& Morin, 2010) and assesses several different parameters of insomnia: difficulty falling/staying asleep and early morning awakenings, sleep satisfaction, degree of impairment with daytime functioning, degree impairments are noticeable, and distress/concern regarding symptoms. Items are measured on a 5-point Likert scale. Cut-off scores of $\geq 8, \geq 15$, and $\geq 22$ are indicative of subthreshold, moderate, and severe insomnia, respectively, whereas in community samples, a cut-off score of $\geq 10$ is indicative of subthreshold insomnia (Morin, Belleville, et al., 2011). Moderate clinical improvement is identified by a reduction of $>7$ points, and marked clinical improvement is identified by a reduction of $>8$ points (Morin, Belleville et al., 2011). As mentioned above, for screening purposes, the cut-off score of $\geq 15$ was used for eligibility for the CBT-I pilot study component, and the cut-off score of $\geq 10$ was used for eligibility for the cross-sectional study component. The ISI demonstrates good internal consistency and test-retest reliability (Edinger \& Carney, 2008), including among samples with a comorbid medical condition (Savard, Savard, Simard, \& Ivers, 2005).

Epworth Sleepiness Scale. The Epworth Sleepiness Scale (ESS; Johns, 1992) is an 8item self-report scale that measures degree of daytime sleepiness. Participants are asked to indicate how likely they are to fall asleep in eight different situations using a 4-point Likert scale 
with responses ranging from 0 (would never doze) to 3 (high chance of dozing). The possible total score ranges from 0 to 24 , and scores $\geq 10$ are indicative of sleep-wake disorders associated with daytime sleepiness such as OSA, hypersomnia, and narcolepsy (Johns, 1991). In contrast, individuals with insomnia typically have very low ESS scores (Johns, 1992). The ESS demonstrates good internal consistency (Cronbach's alpha $=.88$ ), and five-month test-retest reliability $(r=0.82$; Johns, 1992). For the present study, potential participants with an ESS score $\geq 10$ were excluded if they also scored $\geq 5$ on the STOP-BANG Sleep Apnea Questionnaire.

STOP-BANG Sleep Apnea Questionnaire. The STOP-BANG Sleep Apnea Questionnaire (STOP-BANG; Chung et al., 2008; Chung et al., 2012) is an 8-item screening tool for OSA. The name, STOP-BANG, is an acronym for the eight OSA risk factors assessed with the questionnaire: loud snoring, feeling tired in the daytime, having been observed stopping breathing, high blood pressure, BMI, age, neck circumference, and gender (in this measure, gender is a misnomer actually referring to sex). Participants are asked if they experience the following symptoms, to which they respond "Yes" or "No": very loud snoring, feeling tired/fatigued/sleepy during daytime, been observed stopping breathing during sleep, having/being treated for high blood pressure, BMI $>35 \mathrm{~kg} / \mathrm{m}^{2}$, age $>50$ years old, neck circumference $>16$ inches $/ 40 \mathrm{~cm}$, and being male. "Yes" answers are summed for a total score, with scores of 0-2 indicative of low risk, 3-4 indicative of intermediate risk, and 5-8 indicative of high risk of OSA. Although this measure has been validated in the general population, it has not yet been validated among PWH (Taibi, 2013). The STOP-BANG was administered during the telephone screen to anyone with an ESS score $\geq 10$, and participants were excluded who scored $\geq 10$ on the ESS and $\geq 5$ on the STOP-BANG due to the high likelihood of the presence of OSA. 
Duke Structured Interview for Sleep Disorders. The Duke Structured Interview for Sleep Disorders (DSISD; Carney et al., 2008) is a clinician-administered structured interview used to assess for sleep-wake disorders according to DSM-IV-TR and International Classification of Sleep Disorders-second edition (ICSD-2; American Academy of Sleep Medicine, 2005) diagnostic criteria. Separate modules assess four different groups of sleep-wake disorders: insomnia, excessive daytime sleepiness/hypersomnia, circadian rhythm sleep disorders, and parasomnia-related sleep disorders. For the current study, the timeframe for sleep disturbance was altered from past month to past three months to reflect changes to diagnostic criteria for insomnia disorder in DSM-5. There is evidence of acceptable reliability and discriminant validity with the DSISD (Carney et al., 2008; Edinger et al., 2009). Participants diagnosed with any breathing-related sleep disorders (OSA, central sleep apnea, and sleeprelated hypoventilation), or circadian rhythm sleep-wake disorders were excluded from the study. Given that HIV infection may cause enlarged tonsils (Epstein et al., 1995), and lipodystrophy, a side effect of some older cART regimens, may result in fat deposits in the neck, thorax, and abdomen which may impact breathing (Patil, Schneider, Schwartz, \& Smith, 2007), there may be a heightened risk for sleep-related breathing problems among PWH. It is recommended that the presence of OSA be carefully assessed among this population (Smith et al., 2005).

Mini International Neuropsychiatric Interview. The Mini International Neuropsychiatric Interview (MINI; Sheehan et al., 1998) is a clinician-administered semi-structured interview used to assess a wide range of mood, anxiety, substance use, eating, and psychotic disorders according to DSM diagnostic criteria. The initial version of the MINI (MINI 5.0) was designed to assess for symptoms according to DSM-IV (American Psychiatric Association, 1994) 
diagnostic criteria, and demonstrated excellent inter-rater reliability and very good two-day testretest reliability (Sheehan et al., 1998). The most recent version, the MINI 7.0.1, was designed to assess for symptoms according to DSM-5 criteria, and was used in the present study to assess psychiatric status during initial screening and also to diagnose comorbid mental disorders among study participants. Exclusion criteria assessed by the MINI include active suicidal ideation, unmanaged bipolar disorder, severe substance use disorders, and the presence of a psychotic disorder.

Measurement of sleep-related variables. In addition to the ISI, the following measures were used to assess sleep-related variables.

Consensus Sleep Diary. The CSD (Carney et al., 2012) is a prospective, self-report sleep $\log$ on which individuals record information pertaining to each night's sleep and any daytime activities that influence sleep such as naps, caffeine, and alcohol intake. Information collected includes time getting into bed, time sleep is attempted, sleep onset latency, number of awakenings not including final awakening, amount of time spent awake after initially falling asleep, time of final awakening, time of getting out of bed, subjective quality of sleep, and any comments for the researcher. The information provided is used to calculate other parameters that differentiate individuals with insomnia versus normal sleepers, including sleep efficiency (SE), total sleep time, wakefulness after sleep onset, and terminal wakefulness, or amount of time spent awake in bed after the final awakening (Natale et al., 2015). Other sleep parameters identified by the CSD include total time in bed, bedtime and rise time variability, and napping. The CSD distinguishes between individuals with insomnia and good sleepers on several sleep parameters, including sleep onset latency and wakefulness after sleep onset, with cutoffs of 26 minutes and 19 minutes, respectively, yielding the highest sensitivity and specificity in receiver 
operating characteristic curve analyses (Maich, Lachowski, \& Carney, 2018). The primary outcome variables of sleep efficiency and total wake time were calculated using the CSD, with sleep efficiency scores $<85 \%$ and total wake time $>30$ minutes indicative of poor sleep.

Dysfunctional Beliefs and Attitudes about Sleep Scale. The abbreviated Dysfunctional Beliefs and Attitudes about Sleep Scale (DBAS-16; Morin, Vallières, \& Ivers, 2007) is a 16-item self-report measure of disruptive sleep-related attitudes and beliefs. Participants are asked to indicate the degree to which they agree or disagree with each sleep-related statement by circling a number from 1 (strongly disagree) to 10 (strongly agree) on a 10-point Likert scale arranged in visual analog format. Higher scores are indicative of greater dysfunctional beliefs and attitudes. A cutoff mean score of $>3.8$ identifies individuals with clinically significant insomnia, however, it has been proposed that this cutoff may be slightly lower for medical patient populations (Carney et al., 2010). The DBAS-16 has adequate internal consistency and two-week test-retest reliability, and contains four factors representing beliefs and attitudes about the consequences of insomnia, worry about sleep, sleep expectations, and medication (Morin et al., 2007).

Glasgow Sleep Effort Scale. The Glasgow Sleep Effort Scale (GSES; Broomfield \& Espie, 2005) is a 7-item self-report measure of effortful preoccupation with sleep. Participants are presented with statements related to sleep effort and are asked to indicate how true each item is of themselves over the past week with "very much", "to some extent", or "not at all". Total scores range from 0-14, with higher scores indicating greater sleep effort (Fairholme \& Manber, 2014). The GSES has adequate internal consistency, and good discriminant validity, with a cutoff score of 2 correctly identifying $92.1 \%$ of participants with insomnia and $87.3 \%$ of participants who were good sleepers (Broomfield \& Espie, 2005). 
Pre-Sleep Arousal Scale. The 13-item Pre-Sleep Arousal Scale (PSAS-13; JanssonFröjmark \& Norell-Clarke, 2012) is a self-report measure of pre-sleep arousal, and includes 5item and 8-item cognitive and somatic subscales, respectively. Participants are asked to indicate how intensely they experience a range of cognitive and somatic arousal symptoms at bedtime using a Likert scale ranging from 1 (not at all) to 5 (extremely). The PSAS-13 and its subscales have acceptable to good internal consistency, discriminant validity between good sleepers, poor sleepers, and insomnia disorder patients, and fair to moderate convergent validity with measures of anxiety, depression, sleep-related worry, and dysfunctional beliefs about sleep.

Self-Efficacy for Sleep Scale. The Self-Efficacy for Sleep Scale (SE-S; Lacks, 1987) is a 9-item self-report scale that measures confidence for performing sleep-related behaviours on a visual analog scale ranging from "not at all confident" to "very confident". Scores on the visual analog scale range from 0 to 100 . The SE-S has demonstrated acceptable test-retest reliability (Fichten et al., 2001) and good to excellent internal consistency across studies (Edinger et al., 2007; Rutledge, La Guardia, \& Bluestein, 2013).

Fatigue Severity Scale. Fatigue was measured using the Fatigue Severity Scale (FSS; Krupp, LaRocca, Muir-Nash, \& Steinberg, 1989), a 9-item self-report scale designed to measure the impact of fatigue. Participants rate their level of agreement with statements about the impact of fatigue over the past week on a 7-point Likert scale, ranging from 1 (strongly disagree) to 7 (strongly agree). Total possible scores range from 9 to 63, with higher scores indicating greater impact of fatigue. A 7-item version of the FSS (FSS-7) demonstrates better psychometric properties among PWH than the 9-item FSS in terms of goodness-of-fit using the Rasch model (Lerdal et al., 2011), and has excellent internal consistency (Cronbach's alpha $=.92$ ). The FSS-7 excludes the first two items of the FSS, "My motivation is lower when I am fatigued", and 
"Exercise brings on my fatigue". Given the improved psychometric properties of the FSS-7 for PWH, this version was used in the current study.

Measurement of HIV-related clinical variables. CD4 count is an established measure of immune functioning among HIV-positive individuals (Public Health Agency of Canada, 2016). In healthy individuals, CD4 count ranges from 500 to 1,600 cells $/ \mu \mathrm{L}^{3}$. If CD4 count is $<$ 200 cells $/ \mu \mathrm{L}^{3}$, opportunistic infections are likely to occur. In Canada, an AIDS diagnosis is warranted if individuals have been diagnosed with HIV as well as one or more specified indicator diseases, which include diseases such as recurrent bacterial pneumonia, Kaposi's sarcoma, HIV-related encephalopathy, and candidiasis (esophageal, bronchi, trachea, or lungs), among others (Public Health Agency of Canada). According to some guidelines, an HIV viral load $<100,000$ copies $/ \mathrm{mL}$ does not require cART treatment if CD4 count is $>350$ cells $/ \mu \mathrm{L}^{3}$, however if viral load increases to $\geq 100,000$ copies $/ \mathrm{mL}$ and/or CD4 count is $<350$ cells $/ \mu \mathrm{L}^{3}$, standard of care in Canada recommends offering cART (Public Health Agency of Canada). Other guidelines suggest initiating cART regardless of CD4 count or viral load (British Columbia Centre for Excellence in HIV/AIDS, 2018; U.S. Department of Health and Human Services, 2017). Given that standard HIV treatment in Canada involves routine blood tests every three months, participants were asked to provide CD4 count and viral load information from their most recent blood test. CAB members provided feedback on the collection of CD4 count and viral load, recommending not to require a copy of documentation from medical care providers, as this could pose a barrier to participation and communicate distrust. Individuals who had not received a blood test within the past three months were asked to arrange for blood testing in order to provide recent CD4 count and viral load information. The following measures were used to assess remaining HIV-related clinical variables. 
Self-Rating Scale Item. Adherence to cART was measured using the Self-Rating Scale Item (SRSI; Feldman et al., 2013) and the Simplified Medication Adherence Questionnaire (SMAQ; Knobel et al., 2002). The SRSI is a single item self-report medication adherence measure on which participants respond on a 6-point Likert scale to the following question: “thinking about the past 4 weeks, on average, how would you rate your ability to take all your HIV antiretroviral medications as your doctor prescribed?" Response options range from "very poor" to "excellent". The SRSI takes very little time to complete, yet predicts HIV-related clinical variables such as viral load and CD4 count with similar or better accuracy than several other single-item self-report medication adherence measures (Feldman et al., 2013). There is evidence that asking participants to rate their adherence ability results in less over-reporting of adherence estimates than asking about frequency or percentage of adherence (Lu et al., 2008). Similarly, asking participants to recall adherence within a one-month timeframe results in less over-reporting than asking about adherence from seven- and three-day timeframes (Lu et al., 2008).

Simplified Medication Adherence Questionnaire. The SMAQ is a 6-item clinicianadministered questionnaire that examines medication adherence over the past weekend, past week, past three months, and in general. Participants respond to some items dichotomously (e.g. "Yes" or "No"), and others on a Likert scale. Relative to Medication Event Monitoring System (MEMS) electronic pill-cap technology (www.mwvaardex.com), an expensive gold-standard measure of medication adherence, the SMAQ demonstrates $72 \%$ sensitivity and $91 \%$ specificity at identifying non-adherent participants. The internal consistency (Cronbach's alpha) is 0.75 and overall inter-rater reliability is $88.2 \%$. Both the SRSI and SMAQ were used in order to collect adherence data for multiple timeframes. 
HIV Symptom Index. The HIV Symptom Index (HSI; Justice et al., 2001) is a 20-item self-report measure of symptoms associated with HIV and its treatment. Participants indicate the degree to which they have been bothered by each of 20 symptoms over the past month on a 5point Likert scale ranging from 0 (I do not have this symptom) to 4 (It bothers me a lot). Symptoms include fatigue/loss of energy, fevers/chills/sweats, feeling dizzy/lightheaded, pain/numbness/tingling in hands/feet, trouble remembering, nausea/vomiting, diarrhea/loose bowel movements, feeling sad/down/depressed, feeling nervous/anxious, difficulty falling/staying asleep, skin problems, cough, headache, loss of appetite, bloating/gas, muscle aches/joint pain, sexual problems/loss of interest/satisfaction, changes in way body looks/fat deposits, weight loss/wasting, and hair loss.

Medical Outcomes Study Short-Form Health Survey. The Medical Outcomes Study Short-Form Health Survey (SF-36; Ware \& Sherbourne, 1992) is a 36-item self-report survey measuring health-related quality of life (HRQoL). The SF-36 provides eight subscale scores representing the following health concepts: physical functioning, social functioning, bodily pain, role limitations due to physical problems, role limitations due to emotional problems, vitality (energy and fatigue), general mental health, and general health perceptions. Higher scores are indicative of better health. The SF-36 has demonstrated good internal consistency, reliability, and construct validity with HIV-positive samples (Arpinelli, Visona, Bruno, De Carli, \& Apolone, 2000; Hsiung, Fang, Chang, Chen, \& Wang, 2005). Furthermore, the SF-36 has been shown to be responsive to HIV-related health changes such as commencement of cART therapy, number of symptoms, and change in CD4 count and viral load (Carrieri et al., 2003; Saunders \& Burgoyne, 2002). 
Measurement of psychological covariates. Depression, anxiety, stress, alcohol and substance use, and other covariates were assessed using the following measures.

Depression Anxiety Stress Scales. The short-form version of the Depression Anxiety Stress Scales (DASS-21; Henry \& Crawford, 2005) is a 21-item self-report questionnaire that consists of three subscales measuring symptoms of depression, anxiety, and stress, and a total scale measuring general psychological distress. Participants indicate the extent to which each statement applied to them over the past week, using a 4-point Likert scale ranging from 0 (did not apply to me at all) to 3 (applied to me very much, or most of the time). The depression, anxiety, stress, and total scales of the DASS have demonstrated good to excellent internal consistency (Cronbach's alpha $=.88, .82, .90$, and .93 , respectively) and good convergent and discriminant validity with other measures of depression and anxiety in a nonclinical sample (Henry \& Crawford, 2005). The DASS-21 subscales have also demonstrated good to excellent internal consistency (Cronbach's alpha ranging from .87 to .94 ) in a sample of nonclinical volunteers and clinical outpatients (Antony, Bieling, Cox, Enns, \& Swinson, 1998). The full 42item DASS (Lovibond \& Lovibond, 1995) has been used to examine depression, anxiety, and stress among people with insomnia (Espie et al., 2014; Fairholme \& Manber, 2014) and HIV (Landstra, Ciarrochi, Deane, \& Hillman, 2013; Stanley, Sethuramalingam, \& Sathia, 2014). The DASS-21 offers several advantages over the full DASS, including smaller interfactor correlations, a cleaner factor structure, and fewer items (Antony et al., 1998).

Center for Epidemiological Studies in Depression Scale. Depressive symptoms were also measured using the Center for Epidemiological Studies in Depression Scale (CES-D; Radloff, 1977), a 20-item self-report scale designed to measure depression symptoms in the general population. Participants rate how often they experienced each symptom over the past 
week on a 4-point Likert scale ranging from 0 (Rarely or none of the time; less than 1 day a week), to 3 (Most or all of the time; 5 to 7 days a week). Total possible score ranges from 0 to 60 . The CES-D has very good split-half internal consistency in both general and clinical populations (Cronbach's alpha $=.85$ and .90 , respectively), and adequate test-retest reliability, ranging from $r$ $=.51$ for a 2 -week retest period to .67 for a 4-week retest period (Radloff, 1977). The CES-D discriminates well between psychiatric inpatients and the general population, with $70 \%$ of inpatients but only $21 \%$ of individuals in the general population scoring an arbitrary, but now conventional (Henry, Grant, \& Cropsey, 2018) cutoff score of 16 or above. A cutoff score of 23 has been proposed for PWH in a recent validation of the CES-D among a sample of PWH in Ontario (Choi et al., 2015). The CES-D has excellent internal consistency $(\alpha=.93)$, good interrater agreement $(\kappa=.74, S E=0.073)$, excellent sensitivity $(1.0)$, and good specificity $(0.87)$ at a cutoff of 23 among PHW (Choi et al.). However, psychometric limitations of the CES-D have been noted among PWH, including lack of unidimensionality and differential item functioning (Gay, Kottorp, Lerdal, \& Lee, 2016); therefore, caution is warranted when using the CES-D to identify cases of depression among this population. Despite its psychometric limitations, it continues to be among the more common depression screening measures used in HIV research (Simoni et al., 2011), and was included in the current study to facilitate comparison across studies.

Alcohol Use Disorders Identification Test. Alcohol use was assessed using the Alcohol Use Disorders Identification Test (AUDIT; Babor, Higgins-Biddle, Saunders, \& Monteiro, 2001). The AUDIT is a 10-item self-report questionnaire used to screen for hazardous and harmful patterns of alcohol use. Participants are asked to indicate the frequency of various types of alcohol use and related problems on a 5-point Likert scale, with the majority of responses 
ranging from 0 (Never) to 4 (Daily or almost daily). Total possible score ranges from 0 to 40 , and scores $\geq 20$ are indicative of alcohol dependence. For the purpose of this study, individuals who scored $\geq 20$ on the AUDIT and/or who met DSM-5 criteria for severe alcohol use disorder were excluded from the study.

Drug Use Disorders Identification Test. Substance use was assessed using the Drug Use Disorders Identification Test (DUDIT; Berman, Bergman, Palmstierna, \& Schlyter, 2005). The DUDIT is an 11-item self-report questionnaire used to screen for drug-related problems on a 5point Likert scale, with the majority of responses ranging from 0 (Never) to 4 (Daily or almost daily). Total possible score ranges from 0 to 44 , and scores $\geq 25$ are indicative of heavy drug dependence. For the purpose of this study, individuals who scored $\geq 25$ on the DUDIT and/or who met DSM-5 criteria for a severe drug use disorder were excluded from the study.

Socio-demographic covariates. Socio-demographic covariates such as age, sex, gender, sexual orientation, education, annual income, ethnicity, and time since HIV diagnosis were measured via self-report questionnaire.

Therapy Evaluation Questionnaire. Treatment credibility was assessed using the Therapy Evaluation Questionnaire (TEQ; Borkovec \& Nau, 1972), a 7-item self-report questionnaire that measures participants' beliefs about treatment credibility and therapist warmth and competence using a Likert scale. The first five items measure treatment credibility, and the last two items measure therapist warmth and competence. Participants were asked to complete the first five items only, assessing treatment credibility, after their first CBT-I session, and were asked to complete the entire questionnaire after their final CBT-I session.

Treatment Component Checklist. The Treatment Component Checklist (TCC) has been used in previous studies (Carney et al., 2017) to assess therapist fidelity to the treatment protocol, 
and is completed by the therapist after each session. The TCC is a checklist composed of all the relevant therapy components and discussion topics that typically occur during CBT-I. The therapist must indicate, for each session, whether or not each item on the checklist was covered during that specific session. One item on the TCC measures participant adherence to treatment recommendations, including sleep restriction and stimulus control recommendations, using a percentage score. This item was used in the current study to examine adherence to treatment recommendations.

Exit interview. As described above, the overall aim of the exit interview was to explore participants' perceptions of CBT-I and to elicit feedback that could be used to optimize CBT-I for PWH. The exit interview contained 17 questions and was created for the current study with three specific objectives. The first was to elicit feedback about the treatment itself and the research study as a whole, including administrative aspects, in order to determine if and how CBT-I could be specially tailored to make it as acceptable and effective as possible for this population. Feedback on the administrative aspects of CBT-I and the research study could inform related future research, including a randomized controlled trial. The second objective was to examine participants' knowledge about the CBT-I strategies presented during treatment and the extent to which participants found the strategies helpful, in order to determine whether specific strategies are more or less helpful for PWH. The third objective was to complement the quantitative treatment outcome data with qualitative data in order to gain a more complete picture of the impact of CBT-I on sleep-and HIV-related outcomes, as well as any other unintended outcomes that might pertain to the safety of CBT-I among this specific population. The interview questions are presented in Appendix A. Measures that are not copyrighted are found in Appendix B. 


\section{Procedure}

Community advisory board. Prior to finalizing study procedures, a group of individuals living with HIV who also had some familiarity with research or clinical settings was sought to for a small community advisory board $(\mathrm{CAB})$ to inform study methods with the goal of reducing the risk of stigma to participants and increasing cultural sensitivity and community relevance of the project. The $\mathrm{CAB}$ was recruited with the assistance of the primary author's research supervisor and an infectious disease physician who works with PWH and who recommended several individuals who would potentially be interested. In total, four individuals (one man, three women) agreed to participate and met on three separate occasions to provide feedback and guidance on procedures such as financial incentive, exclusion criteria, phrasing of interview questions, recruitment strategies, and feasibility. Each $\mathrm{CAB}$ member was compensated $\$ 50$ for each of the two 2-hour meetings attended, and refreshments were provided at each meeting. Specifically, CAB members provided feedback on collection of HIV-related variables (e.g., CD4 count, viral load, cART medication), incentive amount and format, feasibility of inclusion/exclusion criteria, study name, recruitment strategies, wording of advertisements and consent material, audio recording procedures, potential barriers to participation, and questionnaires/interviews. $\mathrm{CAB}$ members were also encouraged to provide feedback on any other variables they believed may contribute to insomnia among PWH that could be incorporated into the study. CAB feedback was valuable, and incorporated into the study methods.

Telephone screen. A study intake coordinator contacted interested potential participants by telephone to provide detailed information about the study, obtain informed consent for the telephone screen, and conduct the telephone screen to determine preliminary eligibility. This interview assessed inclusion and exclusion criteria mentioned above. As individuals were 
identified during the telephone screen that met the more stringent criteria for being offered CBTI, they were informed about the CBT-I component of the study; however, eligibility for the CBTI component was only confirmed upon completion of the baseline assessment. Potential participants invited to receive CBT-I, therefore, were not randomly selected from the larger sample, but were selected based on being the first 10 participants to be recruited with predetermined CBT-I treatment targets.

During the telephone screen, the presence of insomnia was assessed using the insomnia disorder module of the DSISD (Carney et al., 2008). Insomnia severity was assessed using the ISI (Morin, 1993). Daytime sleepiness was assessed using the ESS (Johns, 1992), and individuals who scored above the recommended cut-off of $\geq 10$ were also administered the STOP-BANG (Chung et al., 2008) to screen for OSA risk factors. Those who scored $\geq 10$ on the ESS and $\geq 5$ on the STOP-BANG were excluded from the study for safety reasons and due to the potential confound of comorbid insomnia and OSA. They were provided brief psychoeducation about OSA and its health risks as well as referral information for a sleep medicine clinic. Those who scored $\geq 10$ on the ESS but $<5$ on the STOP-BANG were not excluded. Individuals who reported using alcohol or substances on a "daily or almost daily" basis were also administered the applicable alcohol use disorders and/or substance use disorders modules from the MINI (Sheehan et al., 1998) and were excluded if they met DSM-5 diagnostic criteria for severe alcohol or substance use disorder.

Potential participants were asked whether they were willing to provide HIV viral load and $\mathrm{CD} 4$ count information during the baseline assessment. In order to decrease participant burden, participants were not required to provide medical documentation of HIV viral load and CD4 count. Participants who had not had a blood test within the past three months were asked 
during the telephone screening to obtain updated results from a health care provider before the baseline assessment. Participants who undergo regular blood testing every three months as part of their standard treatment and who have recent viral load and CD4 count information began the study immediately. Participants who do not undergo regular blood testing every three months and who had to schedule an appointment for blood testing began the study once they receive updated viral load and CD4 count information.

Potential participants who met eligibility criteria after the telephone screen received instructions on filling out the CSD and were scheduled for a baseline assessment. They were asked to provide verbal consent to be contacted with appointment reminders either via email or phone. Participants who could not print out the CSD at home were offered the option of having it mailed via Canada Post or coming to the HIV Prevention Lab to pick it up in person.

Baseline assessment. Potential participants returned the completed sleep diary to Ryerson University in person, provided informed consent, and completed a baseline assessment consisting of: 1) a clinician-administered interview to screen for a wide range of sleep and mental disorders using the DSISD and MINI; and 2) a computer-administered questionnaire package to measure sociodemographic variables as well as sleep-, HIV-, and mental healthrelated variables. All baseline assessments were conducted by a clinical psychology doctoral student (T. Tulloch). Final eligibility was determined after the clinician-administered interview, but before the computer-administered questionnaire package. If participants no longer met criteria for the study during or after the clinician-administered interview, but had provided informed consent, the assessment was terminated upon discovery of ineligibility and they received the incentive. Upon completion of the assessment, participants were given the choice of receiving either: 1) \$50; or 2) \$25 along with a copy of Quiet your Mind and Get to Sleep 
(Carney \& Manber, 2009), a self-help book for people with insomnia based on CBT-I principles. Participants in the CBT-I pilot study did not receive an incentive immediately after the assessment, but received $\$ 60$ upon completion of CBT-I and the posttreatment assessment. The community advisory board approved the incentive options. Participants not in the CBT-I pilot study (i.e., participation completed) were informed about a future knowledge transfer and exchange (KTE) event at which the study results would be shared with the community. Participants interested in being contacted about this event in the future provided written informed consent along with their preferred contact information.

CBT-I pilot study. Participants in the CBT-I pilot study received a standard protocol of individual, bi-weekly, 4-session CBT-I administered by a clinical psychology doctoral student (T. Tulloch). Some potential barriers to participation include low socioeconomic status and work/child care schedules. In order to reduce the potential impact of these barriers, assessment and treatment appointments were scheduled flexibly, with evening and weekend options available. Participants monitored sleep-related variables using the CSD throughout the study, and completed the ISI and MQS at each biweekly therapy session. Participants were monitored at each session for any sign of clinical worsening, which, for the purpose of this study, was defined as an increase of $50 \%$ on the ISI from baseline levels or sleep efficiency $>95 \%$.

The goal of CBT-I is to modify cognitive and behavioural factors that perpetuate insomnia through several specific techniques: sleep education, sleep restriction, stimulus control, cognitive restructuring, and sleep hygiene. Sleep education involves providing information sleep norms, circadian rhythm, sleep drive, and sleep deprivation. The goal of sleep education is to correct misconceptions about sleep, set realistic expectations, and provide a treatment rationale (Edinger \& Carney, 2008). Sleep restriction involves restricting the amount of time an individual 
spends in bed to the amount of time they report sleeping each night on average on the CSD by providing a personalized "time in bed" prescription. The goal of sleep restriction is to compress an individual's sleep into a shorter window in order to improve sleep quality. This also serves to increase sleepiness, which, in turn, improves sleep quality and decreases the amount of time spent falling asleep and waking up during the night (Manber \& Carney, 2015). Stimulus control involves implementing behavioural strategies to strengthen the association between the bed and sleep. For people with insomnia, the bed is often associated with anxiety, frustration, fear, or wakeful activities such as reading, checking emails, and spending time on social media. The goal of stimulus control is re-associate the bed with sleep by moving all activities outside of the bedroom except for sleep and sex. Stimulus control guidelines include going to bed only when sleepy, getting out of bed and the bedroom if unable to sleep, and using the bed only for sleep and sex (Manber \& Carney). Other helpful behavioural strategies include avoiding napping and waking up and getting out of bed at the same time every morning. These help to increase sleep drive, and maintain a stable circadian rhythm, respectively. Cognitive restructuring involves identifying and modifying unhelpful thinking patterns that cause anxiety and other negative emotions that interfere with sleep. Some cognitive restructuring strategies include challenging unhelpful thoughts using a thought record, therapist-led Socratic questioning, conducting behavioural experiments to test predictions, scheduling constructive worry time, and conducting a cost/benefit analysis (Manber \& Carney). Sleep hygiene refers to behaviours that may impact sleep, such as caffeine consumption, alcohol and substance use, timing of arousing/alerting activities such as exercise and eating, and environmental factors such as light, noise, and temperature (Manber \& Carney). 
Session 1. During the first session, and every session thereafter, participants completed the ISI and MQS, and the therapist reviewed data from the CSD verbally with the participant. During the first session, the therapist presented sleep education and introduced the concepts of sleep restriction and stimulus control, then provided a personalized "time in bed" prescription. The therapist used a motivational interviewing approach (Miller \& Rollnick, 2002) to build motivation and reduce resistance when needed. Participants were asked to follow the sleep restriction and stimulus control guidelines while implementing their "time in bed" prescription.

Session 2. During the second session, the therapist reviewed progress and adherence and recommended changes to the prescription based on CSD data. Cognitive restructuring strategies were introduced, including the thought record and constructive worry exercise. Information was provided about causes of fatigue and the misattribution of fatigue on sleep, and participants were provided information on managing fatigue using cognitive and behavioural strategies. These included challenging unhelpful thoughts about fatigue and engaging in moderate levels of activity. Participants were asked to continue following sleep restriction and stimulus control guidelines while implementing their prescription. They were also asked to practice new cognitive restructuring strategies.

Session 3. During the third session, the therapist reviewed progress and adherence and recommended changes to the prescription based on CSD data. During this session, information from the previous two sessions was reviewed and the therapist assisted in troubleshooting any difficulties with strategies, and reinforcing successful implementation of strategies. Participants were asked to continue following sleep restriction and stimulus control guidelines while implementing their prescription and practicing cognitive restructuring strategies. As a relapse 
prevention strategy, participants were also asked to write a letter to their future selves, imagining what they would say to themselves if they found their insomnia had returned.

Session 4. Similar to Session 3, during the fourth and final session, the therapist reviewed progress and adherence and recommended changes to the prescription based on CSD data. During this session, information from the previous sessions was reviewed and the therapist assisted in troubleshooting any difficulties with strategies, and reinforcing successful implementation of strategies. The therapist reviewed the letters participants wrote to their future selves, and instructed participants how to calculate their sleep efficiency scores (time spent sleeping divided by total time in bed), which is used to determine "time in bed" prescriptions. Participants were asked to continue following sleep restriction and stimulus control guidelines while implementing their prescription and practicing cognitive restructuring strategies. The therapist reminded participants about the exit interview that would take place during the posttreatment assessment and encouraged them to be as forthcoming as possible. Given that the post-treatment assessment occurs approximately two months after the baseline assessment, and standard of care for CD4 and viral load testing is every three months, participants were reminded during the final CBT-I session to undergo another blood test and provide these values during the post-treatment assessment, or as soon as possible thereafter. Participants were asked to monitor sleep using the CSD for an additional two weeks in preparation for the posttreatment assessment.

Posttreatment assessment. Participants returned to the HIV Prevention Lab with the completed sleep diary to complete the post-treatment assessment consisting of: 1) the same computer-administered questionnaire package as at baseline; and 2) an exit interview. A research assistant who did not provide CBT-I administered the exit interview during the posttreatment assessment. The interview took approximately 30-40 minutes to administer. Exit interviews were 
audio recorded and later transcribed by a research assistant using a word processor. Participants were reminded immediately prior to the exit interview not to use any personally identifiable information such as surnames, addresses, birth dates, jobs, or any other unique identifying information that could be linked back to them or anyone else. Participants received $\$ 30$ per assessment (baseline and posttreatment) for a total of $\$ 60$, paid in full upon completion of the study. They were also provided with two transit tokens after each in-person visit in order to reduce transportation cost as a barrier to participation.

\section{Research Design}

The current study used an interrupted time-series design to examine change in insomnia severity, sleep efficiency, and total wake time among CBT-I participants, measured using mean ISI score, sleep efficiency, and total wake time, respectively. With interrupted time-series singlecase design, multiple baseline data serve as a comparison condition for the data collected after the intervention is administered. Multiple baseline design demonstrates if change occurs when, and only when, the intervention is administered (Kazdin, 1982).

Some features of single-case research designs that reduce threats to internal validity include: 1) objective data; 2) continuous assessment; 3) stability of problem; 4) immediate and marked effects of the intervention; and 5) multiple cases (Kazdin, 1982). The use of objective data, including self-report inventories and observed/reported behaviour, provide a basis for determining if, and to what extent, change occurred. The use of continuous assessment before and after the intervention vs. comparing pre- and post-intervention scores provides more nuanced information about patterns of change and reduces the likelihood of threats to internal validity. Similarly, continuous assessment before the intervention is administered provides the opportunity to demonstrate stability of the problem prior to the intervention. The immediacy and 
magnitude of change resulting from the intervention are unknown at the outset, but provide further support that the intervention is responsible for the change. Finally, including multiple single-case analyses versus relying on one example provides more robust support for the intervention's efficacy (Kazdin, 1982).

The current study incorporated each of the above recommended features, when possible, to reduce threats to internal validity. Objective data were used to measure the effects of CBT-I, including a self-report questionnaire and prospectively recorded self-reported sleep data. Baseline data was collected for each individual three times for the primary outcome variable, insomnia severity, and twice for the other outcome variables of interest, sleep efficiency and total wake time. Insomnia disorder is known to be a chronic condition (American Psychiatric Association, 2013; Jansson-Fröjmark \& Linton, 2008) and all participants reported experiencing insomnia for at least three months duration prior to study inclusion. The multiple baseline measurements were also intended to demonstrate stability of insomnia prior to provision of CBTI. Although the immediacy and magnitude of CBT-I impact was not known prior to carrying out the study, CBT-I is associated with medium to large effect sizes in the general population (Okajima, Komada, \& Inoue, 2011). Data from 10 participants were examined separately, further reducing threats to internal validity.

In addition to the single-case interrupted time-series design used to examine effects of CBT-I, an exit interview was used to explore participant experiences while receiving CBT-I and to elicit feedback that will be used to optimize treatment of insomnia among PWH. This mixed methods design, incorporating both quantitative and qualitative approaches to address the research questions, is particularly well suited for the study, given its goals of examining quantitative data such as the impact of CBT-I on sleep- and HIV-related variables, and 
qualitative data such as subjective participants' experiences that may be used to inform and improve future CBT-I interventions with PWH. The rationale for employing a mixed methods design in the current study is to arrive at as detailed an understanding as possible about how CBT-I impacts PWH and how it could be tailored in the future to provide even greater benefit to this specific population. A mixed methods approach incorporates information from complementary sources, permitting a more thorough examination of the research questions and a more complete picture of the results than either quantitative or qualitative approaches alone (Denscombe, 2008; Tariq \& Woodman, 2013).

\section{Data Analysis}

Basic univariate and bivariate descriptive analyses were used to explore features of insomnia in this population, including the characteristics, comorbidity, and covariates.

Visual inspection. Given the small sample size of the CBT-I pilot study $(n=10)$, and the use of single-case interrupted time-series design, change in the primary outcome variables of ISI, sleep efficiency, and total wake time was evaluated using visual inspection, the recommended analytic technique for single-case research designs (Busk \& Marascuilo, 2015; Kazdin, 1982). With visual inspection, scores are plotted on a line graph and changes in mean, level, and trend, as well as latency of change are used to identify reliable or consistent intervention effects. A change in mean refers to the difference between the mean score of the baseline phase and the mean score of the phase after the intervention is implemented. A change in level refers to the difference between the last data point prior to the intervention being implemented and the first data point immediately afterward. A change in trend refers to a change in the slope of plotted scores in the baseline versus intervention phases. Finally, latency of change refers to how soon after the intervention is implemented that change is observed (Kazdin, 1982). Change over time 
on primary sleep-related outcomes of insomnia severity, sleep efficiency, and total wake time were examined using visual inspection of data collected during the telephone screen (insomnia severity only), baseline assessment, each treatment session, and posttreatment assessment.

Determining whether an intervention has resulted in reliable change based on a visual inspection of plotted data is a subjective endeavor. For this reason, descriptive aids are applied to mitigate bias in the evaluation process. Descriptive aids include plotting mean and standard deviations as well as celeration lines-lines identifying the slope of data - alongside the data (Kazdin, 1982). In the current study, two separate graphs were plotted for each participant for each outcome variable. While both graphs for each participant for any given variable include the same raw scores separated by a line indicating when treatment was first administered, each includes different visual aids. To identify reliable change based on mean scores, the two-standard deviation band method, also known as the Shewart chart method (Bloom \& Fisher, 1982), was applied. With this method, the baseline mean score \pm 2 standard deviations is plotted, and carried over into the following phase. The visual aid on the first of each pair of graphs, therefore, is the plotted $M$ and $\pm 2 S D$ of the entire baseline period, up until the participant received the first CBT-I session. Using this visual aid, which accounts for variability in baseline data (Nourbakhsh \& Ottenbacher, 1994), any scores beyond \pm 2 standard deviations from the baseline mean are considered evidence of reliable change. One recommended marker of reliable change is if two consecutive scores fall outside \pm 2 standard deviations (Gottman \& Leiblum, 1974)

To identify reliable change based on change in slope, celeration lines are plotted in each phase (e.g., baseline and intervention), then baseline celeration lines are extended into the intervention portion of the graph to serve as a visual comparison by which the intervention celeration line slope can be measured. For example, if a baseline celeration line is extended into 
the intervention phase, and the intervention celeration line does not appear to be simply an extension of the baseline celeration line, but has a different slope based on visual inspection, then a conclusion of reliable change may be justified. This technique is known as the split middle technique (White, 1974). Level and immediacy of change are easily identified without additional visual aids.

Exploratory inferential analyses. Given the small sample size $(n=10)$, the predicted low power to detect large effects, and the preliminary state of research examining efficacy of CBT-I among PWH, no hypotheses were made about the statistical significance of pre- to postreatment change in primary outcome variables. Exploratory analyses were nonetheless conducted to examine whether mean insomnia severity, sleep efficiency, and total wake time at posttreatment were significantly different than mean scores at baseline. Bias-corrected bootstrapping was applied to a series of paired sample $t$-tests to address non-normal distribution expected in the small data set. For the bootstrapping analyses, 5000 samples were run based on a $95 \%$ confidence interval.

Thematic analysis. Undergraduate and graduate level research assistants transcribed qualitative data from the exit interviews. Two clinical psychology doctoral level students (T. Tulloch and C. Mutschler) independently coded data from the transcribed exit interviews, and identified themes using thematic analysis (Braun \& Clarke, 2006). This occurred through an iterative process, in which the coders met to review the initial codes and themes together, making revisions as needed until reaching a consensus about the themes. Although interview questions were structured to elicit information pertaining to three specific objectives, the goal of thematic analysis was not to simply replicate these categories. 
Based on recommendations put forth by Braun and Clarke (2006), the coders first decided that themes would include any topic relevant to the research question(s) that were mentioned even once. Topics not relevant to the research question were included as themes if they were mentioned more than once. Second, given the interrelated nature of the three main goals within an overall aim of optimizing CBT-I for PWH, the coding and themes generated reflected the entire interview rather than just the specific question being asked. In this way, themes provided a description of the overall interview. Third, the coders decided to use a theoretical rather than inductive approach to thematic analysis, given that the questions were driven by a specific analytic goal of improving the treatment and implementation methods, rather than understanding participant experiences inductively. Fourth, given that the goals of the present study were to understand participants' experiences rather than explore underlying ideologies, assumptions, and ideas, themes were identified at the semantic level rather than at a latent/interpretive level. Fifth, a pragmatic worldview was chosen to frame the current study, given its solution-focused, mixed methods approach, and its assumption that reality exists both in the external world and within the mind.

Consistent with the six phases of thematic analysis recommended by Braun and Clarke (2006), the coders first became familiar with the data by reviewing the transcripts several times and making notes about potential themes or areas of interest. Next, codes were generated independently by each coder to identify interesting features of the data. Codes typically identify the most basic elements of the transcript that are meaningful to the research question, and are typically on a more specific level than themes, which are conceptually broader. Codes were collated with corresponding extracts from the transcript. Next, the coders sorted codes into candidate themes and sub-themes, and collated them into categories under their corresponding 
themes. A thematic map is a useful visual tool for collating codes into themes and sub-themes, and was used to inform the next phase, which involved reviewing and refining the candidate themes. This involved first reviewing the coded extracts to ensure coherence among extracts in each of them, then reviewing the themes themselves and the thematic map to ensure an accurate representation of the interview data overall. During this phase, transcripts were reviewed for any data that was missed in earlier coding. At this phase, the iterative process occurred, with codes, themes, and sub-themes refined several times. Next, the coders defined and refined themes by describing each theme and associated sub-themes in detail and how they fit into the overall picture being portrayed by the thematic analysis. Finally, results from the thematic analysis were described below, including transcript extracts to clarify and exemplify each theme. The use of thematic analysis is consistent with the pragmatic lens that framed the study, in that it provides access to participants' internal realities with which they understand and interpret the world, all within the context of an external reality in which the effects of CBT-I are quantified.

\section{Results}

\section{Recruitment}

A total of 181 individuals responded to print, online, and word-of-mouth advertising, of which 142 (78.4\%) were successfully contacted and screened via telephone. Of those screened, $84(59.2 \%)$ were eligible and invited to participate in the study. The most common reasons for ineligibility during the phone screen were having a diagnosis of obstructive sleep apnea (OSA) or suspected OSA $(n=20 ; 14.1 \%)$, having subthreshold insomnia severity $(n=18 ; 12.7 \%)$, and reporting a psychiatric diagnosis of unmanaged bipolar disorder, schizophrenia, psychosis, or severe substance use disorder $(n=9 ; 6.3 \%)$. Other reasons for ineligibility included not having an HIV diagnosis ( $n=4 ; 2.8 \%$ ), being unable to attend the assessment due to living too far from 
Toronto $(n=5 ; 3.5 \%)$, frequent travel between time zones $(n=1 ; 0.7 \%)$, and not being able to read or communicate in English $(n=1 ; 0.7 \%)$. Participant flow and reasons for exclusion are reported in Figure 1. Of the 84 individuals invited for a baseline assessment, $53(63.1 \%)$ attended the baseline assessment and provided verbal and written informed consent, 22 (26.2\%) withdrew prior the assessment due to health, work, personal, or other circumstances, eight (9.5\%) did not show up and/or could not be contacted, and one (1.2\%) was excluded for not completing the sleep diary after two attempts. Data from five participants $(6.0 \%)$ who attended the baseline assessment were either incomplete or not collected due to no longer meeting inclusion criteria after providing informed consent, resulting in a sample of 48 participants used for data analyses. All baseline assessments were conducted between October, 2016 and April, 2018. CBT-I was offered to eligible participants at baseline until a sample $n=10$ was attained. A total of 14 participants ended up being offered CBT-I in order to achieve the desired sample size. One participant attended one session, then became ill and withdrew. Two participants declined due to the lengthy time commitment involved, and another withdrew prior to initiating CBT-I due to interfering PTSD symptoms. This second participant expressed concern about CBT-I increasing PTSD symptomology. In sum, out of the initial 142 potential participants who were screened via telephone, 59.2\% met eligibility criteria, 37.3\% attended the baseline assessment, and $33.8 \%$ met full criteria at baseline and participated in the study. Furthermore, $78.6 \%$ of participants offered CBT-I accepted the offer, and there was an attrition rate of $9.1 \%(n=1)$ among participants who received at least one session of CBT-I.

\section{Features of Insomnia Among PWH}

One goal of the current study was to describe the features of insomnia among PWH, specifically, the characteristics, comorbidity, and covariates. All descriptive statistics for sleep, 


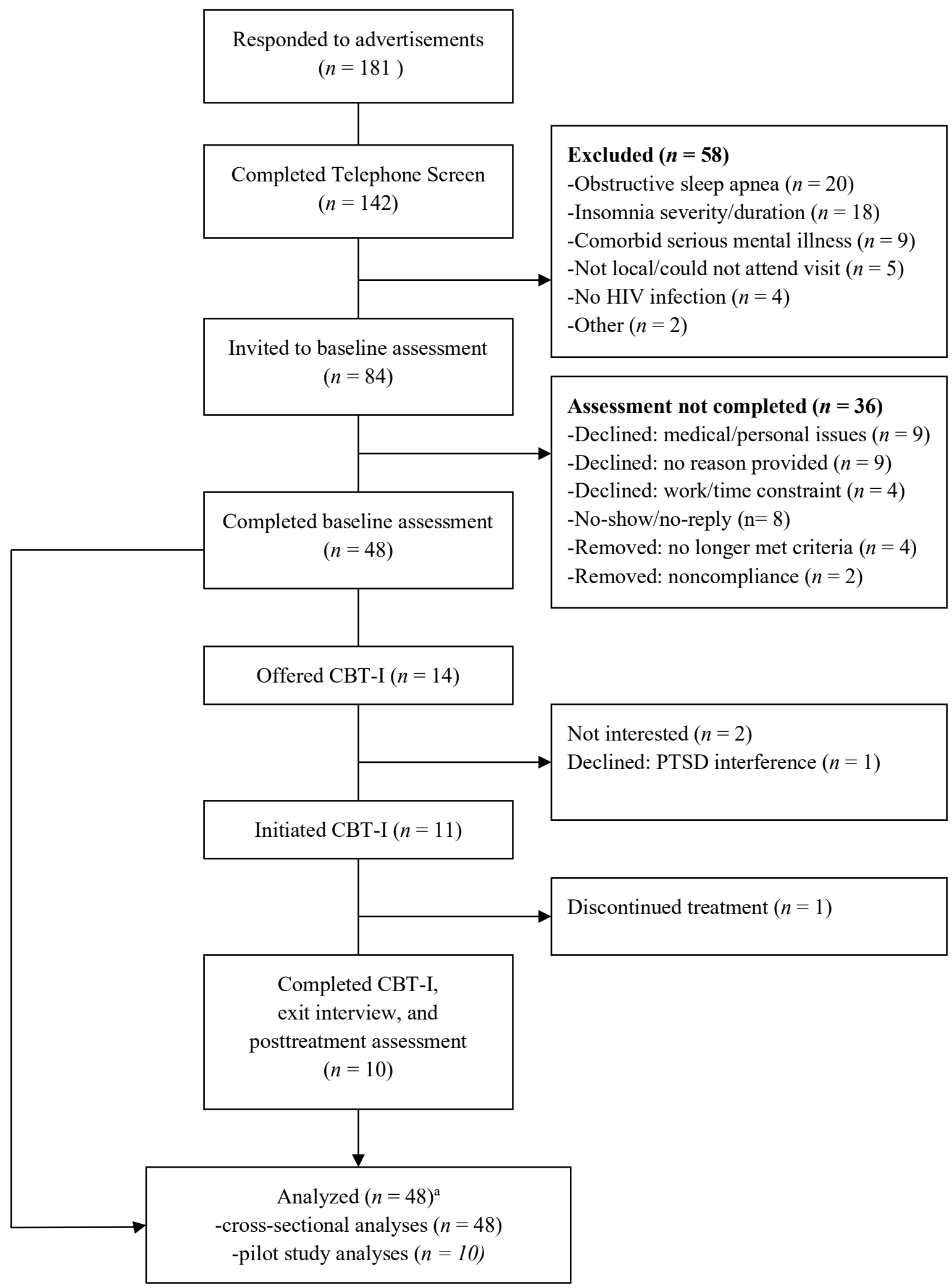

Figure 1. Recruitment response rate and flow of participants through the study. CBT-I =

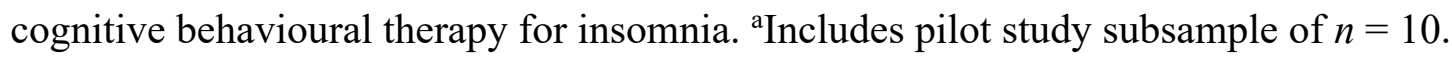


HIV, and other clinical variables in the larger sample are presented in Table 2.

Characteristics of insomnia. Participants reported living with insomnia for a mean of 8.2 years $(S D=8.6)$, with $29.2 \%$ of participants reporting that their insomnia predated their HIV diagnosis. The majority (54.2\%) reported that they were diagnosed with HIV prior to experiencing insomnia, with the mean number of years between HIV diagnosis and insomnia onset among these participants being $11.3(S D=7.3)$. Another $12.5 \%$ reported that insomnia onset coincided with their HIV diagnosis.

Insomnia Severity Index. Using ISI cutoff criteria proposed by Morin, Belleville, et al. (2011), 39.6\% of the sample scored in the range indicative of subthreshold insomnia, $52.1 \%$ scored in the range indicative of moderate insomnia, and $8.3 \%$ scored in the range indicative of severe insomnia. The mean ISI total score was $16.7(S D=3.9)$, falling in the range indicative of moderate insomnia.

Consensus Sleep Diary. Prospectively monitored sleep variables were collected over two weeks using the CSD, therefore, the following results reflect two-week means. The mean sleep onset latency was 44.7 minutes $(S D=40.2)$, with $52.1 \%$ of participants taking $<30$ minutes to fall asleep, 25\% taking 30-59 minutes, $12.5 \%$ taking 60-89 minutes, and 10.4\% taking 90 minutes or more. A total of 58.3\% reported taking 26 minutes or more to fall asleep, which has been identified as the optimal cutoff for identifying poor vs. good sleepers (Maich et al., 2018). The mean number of awakenings was $2.4(S D=1.6)$, and the mean wakefulness after sleep onset was 55.4 minutes $(S D=55.3)$, with $37.5 \%$ of participants being awake for less than 30 minutes, $29.2 \%$ being awake for $30-59$ minutes, $12.5 \%$ being awake for $60-89$ minutes, and $20.8 \%$ being awake for 90 minutes or more. A total of $75 \%$ reported being awake for at least 19 minutes after initial sleep onset, which has been identified as the optimal cutoff for identifying poor vs. good 
Table 2

Clinical Variables of Full Sample at Baseline $(\mathrm{n}=48)$

\begin{tabular}{|c|c|c|c|}
\hline Variable & $\%(n)$ & $M(S D)$ & $\alpha$ \\
\hline \multicolumn{4}{|c|}{ Sleep-related variables } \\
\hline \multicolumn{4}{|c|}{ Consensus Sleep Diary (2-week period) } \\
\hline SOL (min) & & $44.7(40.2)$ & \\
\hline NWAK & & $2.38(1.59)$ & \\
\hline WASO (min) & & $55.4(55.3)$ & \\
\hline TWASO (min) & & $44.6(35.1)$ & \\
\hline $\mathrm{TIB}(\min )$ & & $531.1(60.5)$ & \\
\hline TST (min) & & $353.0(87.7)$ & \\
\hline TWT (min) & & $144.7(67.5)$ & \\
\hline SE & & $0.70(0.15)$ & \\
\hline Daytime napping (min) & & $26.4(34.2)$ & \\
\hline ISI & & $16.7(3.9)$ & $.78^{\mathrm{b}}$ \\
\hline ESS & & $8.1(4.7)$ & \\
\hline DBAS-16 & & $6.2(1.5)$ & .86 \\
\hline GSES & & $8.2(3.4)$ & .80 \\
\hline PSAS-13 & & $31.9(8.9)$ & .87 \\
\hline cognitive subscale & & $16.7(4.9)$ & .89 \\
\hline somatic subscale & & $15.2(6.0)$ & .85 \\
\hline SE-S & & $47.4(17.0)$ & .77 \\
\hline FSS-7 & & $4.3(1.4)$ & .89 \\
\hline \multicolumn{4}{|l|}{ HIV-related variables } \\
\hline CD4 count & & $637.8(238.4)$ & \\
\hline Over $350 / \mu \mathrm{L}^{3}$ & $79.2(38)$ & & \\
\hline $200-350 / \mu \mathrm{L}^{3}$ & $4.2(2)$ & & \\
\hline$<200 / \mu \mathrm{L}^{3}$ & $4.2(2)$ & & \\
\hline Can't remember/not told & $12.6(6)$ & & \\
\hline Viral load & & $525.2(3520.7)$ & \\
\hline Undetectable & $87.5(42)^{\mathrm{a}}$ & & \\
\hline$<100,000$ copies $/ \mathrm{mL}$ & $10.5(5)$ & & \\
\hline Waiting for results & $2.1(1)$ & & \\
\hline \multicolumn{4}{|l|}{ SRSI (cART adherence) } \\
\hline Excellent & $66.7(32)$ & & \\
\hline Very good & $27.1(13)$ & & \\
\hline Good & $6.3(3)$ & & \\
\hline SMAQ: Adherent to cART & $47.9(23)$ & & \\
\hline
\end{tabular}

(continued) 


\begin{tabular}{lcc} 
Variable & $\%(n)$ & $M(S D)$ \\
\hline HIV-related variables (continued) & & \\
HSI-bothersome & $10.3(4.4)$ & \\
SF-36 & & \\
Physical functioning & $84.1(18.0)$ & .86 \\
Role limitations-physical & $58.8(40.8)$ & .85 \\
Role limitations-emotional & $51.8(46.5)$ & .91 \\
Energy/fatigue & $41.5(19.3)$ & .81 \\
Emotional wellbeing & $63.0(22.1)$ & .89 \\
Social functioning & $56.8(24.2)$ & .80 \\
Pain & $65.7(24.3)$ & .88 \\
General health & $59.8(18.2)$ & .64 \\
& & \\
Psychological variables & & .91 \\
DASS-21 & & .91 \\
Depression & $37.1(22.6)$ & .66 \\
Anxiety & $12.2(10.1)$ & .84 \\
Stress & $9.2(6.9)$ & .91 \\
CES-D & $15.6(9.3)$ & \\
AUDIT & $19.8(11.3)$ & \\
DUDIT & $5.9(6.3)$ &
\end{tabular}

Note. $\alpha=$ Cronbach's $\alpha$; AUDIT = Alcohol Use Disorders Identification Test; CES-D = Center for Epidemiological Studies in Depression Scale; DASS-21 = Depression, Anxiety, Stress Scales; DBAS-16 = Dysfunctional Beliefs About Sleep Scale; DUDIT = Drug Use Disorders Identification Test; ESS = Epworth Sleepiness Scale; FSS-7 = Fatigue Severity Scale; GSES = Glasgow Sleep Effort Scale; HSI-bothersome = number of bothersome symptoms reported on HIV Symptom Index; ISI = Insomnia Severity Index; NWAK = number of awakenings; PSAS13 = Pre-Sleep Arousal Scale; SE = sleep efficiency; SE-S = Self-Efficacy for Sleep Scale; SF$36=$ Medical Outcomes Study Short-Form Health Survey; SMAQ = Simplified Medication Adherence Questionnaire; SOL = sleep onset latency; SRSI = Self-Rating Scale Item; TIB = time in bed; TST = total sleep time; TWASO = terminal wake after sleep onset; TWT = total wake time; WASO = wake after sleep onset.

${ }^{a}$ Viral load $M, S D$ should be interpreted with caution given high frequency of reported undetectable viral loads.

bCronbach's $\alpha$ calculated for ISI items 2-5 only as items 1a-c measure different types of sleep disturbance and internal consistency not anticipated. 
sleepers (Maich et al., 2018). Only six participants scored below both the sleep onset latency and wakefulness after sleep onset cutoffs of 26 and 19 minutes, respectively. The mean time in bed was 531.1 minutes $(S D=60.5)$, or 8 hours, 51 minutes. The mean total sleep time was 353.0 minutes $(S D=87.7)$, or 5 hours, 53 minutes, with $20.8 \%$ of participants sleeping $<5$ hours per night, $29.2 \%$ sleeping 5 to 5.9 hours, $37.5 \%$ sleeping 6 to 6.9 hours, and $12.6 \%$ sleeping for 7 hours or more. The mean total wake time was 144.7 minutes $(S D=1.1)$, or 2 hours, 25 minutes. The mean sleep efficiency in the sample was $70 \%(S D=15 \%)$, well below the cutoff of $85 \%$ used to differentiate individuals with insomnia from good sleepers.

CBT-I treatment targets. Another goal of the current study was to identify the presence of relevant insomnia treatment targets, such as daytime napping, extended time in bed, engaging in non-sleep activities in the bedroom, and experiencing worry, anxiety, and/or physical tension when attempting to sleep (Smith \& Perlis, 2006). Over the two-week monitoring period, participants reported napping for an average of 26.4 minutes per day $(S D=34.2)$. They reported spending an average of 44.6 minutes awake in bed after their final awakening $(S D=35.1)$. Overall, participants spent an average of 531.1 minutes $(S D=60.5)$ in bed each night and only slept for 353.0 minutes $(S D=87.7)$ of that time, indicating that they spent much more time in bed than they did actually sleeping. Worry, anxiety, and physical tension at bedtime was measured using the PSAS-13, and the average score $(M=31.9, S D=8.9)$ is comparable to community samples of individuals with insomnia $(M=30.2, S D=8.4$; Jansson-Fröjmark \& Norell-Clarke, 2012). For comparison, the mean PSAS-13 score for good sleepers has been reported in other studies as $18.3(S D=4.7$; Jansson-Fröjmark \& Norell-Clarke). Engagement in non-sleep activities in the bedroom was not quantified in the current study; however, many participants reported watching television, browsing the Internet, or engaging in other daytime 
activities while in bed. Together, these findings demonstrate the presence of relevant CBT-I treatment targets among the current sample.

Insomnia comorbidity. The majority of participants had comorbid psychiatric disorders, with $54.2 \%(n=26)$ being diagnosed with at least one non-sleep psychiatric disorder, $54.2 \%(n=$ 26 ) being diagnosed with at least one sleep disorder in addition to insomnia, and $72.9 \%(n=35)$ being diagnosed with at least one of any psychiatric disorder (including sleep disorders). The most prevalent comorbid non-sleep psychiatric disorder was social anxiety disorder, present in $27.1 \%$ of the sample $(n=13)$, followed by alcohol use disorder, severe, present in $16.7 \%$ of the sample $(n=8)$, and major depressive disorder, present in $12.5 \%$ of the sample $(n=6)$. The most prevalent comorbid sleep disorder was OSA, being identified in $33.3 \%$ of the sample $(n=16)$. As OSA cannot be diagnosed without polysomnography, which was not part of the study protocol, it was identified as being likely present using the DSISD, but was not diagnosed. Although the presence of OSA was one of the study exclusion criteria, and participants were excluded if this was identified during the phone screen, participant data were retained if OSA was identified during the baseline assessment. This decision was made based on the high prevalence of OSA identified in this population, and also given the pragmatic nature of the study. Overall comorbidity frequency is presented in Figure 2, and the frequency of each comorbid psychiatric disorder is presented in Figure 3. The number of comorbid disorders was not significantly correlated with indicators of insomnia such as ISI, sleep efficiency, and total wake time; however, symptoms of depression, anxiety, and stress as measured by the CES-D, DASSanxiety subscale and DASS-stress subscale were associated with insomnia severity, as mentioned below. 


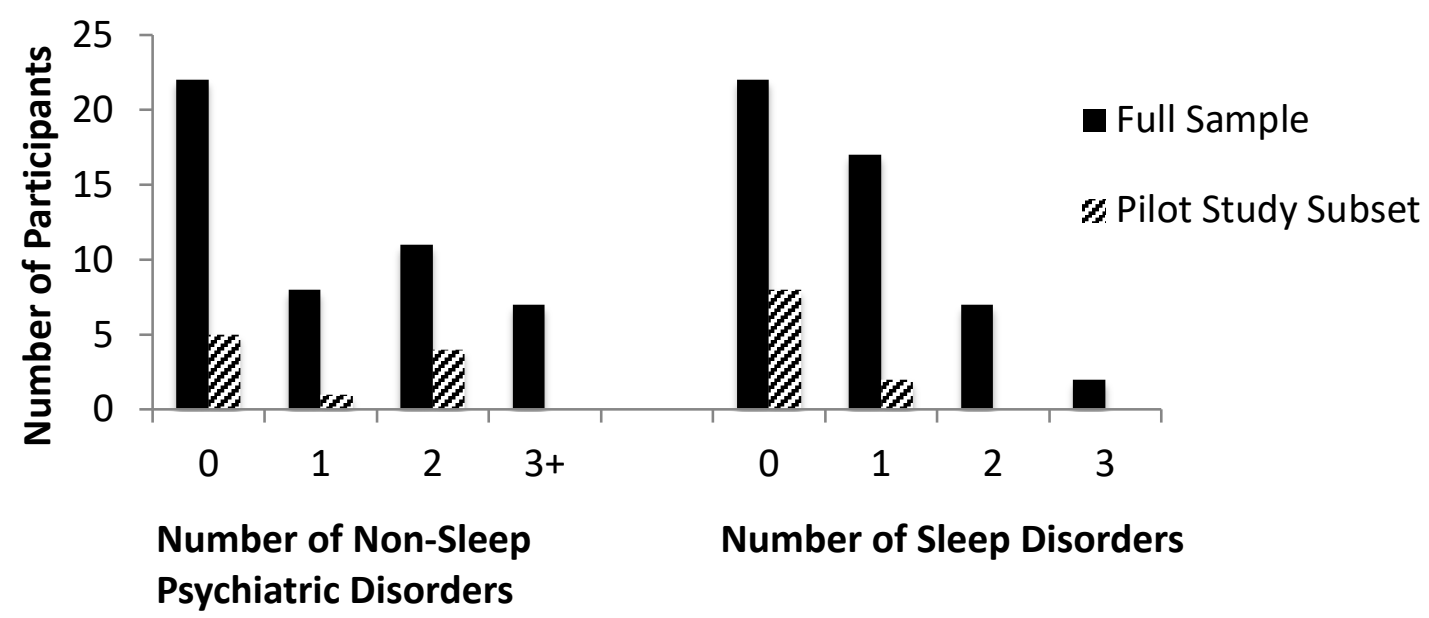

Figure 2. Number of comorbid psychiatric disorders (non-sleep and sleep) diagnosed at baseline. 


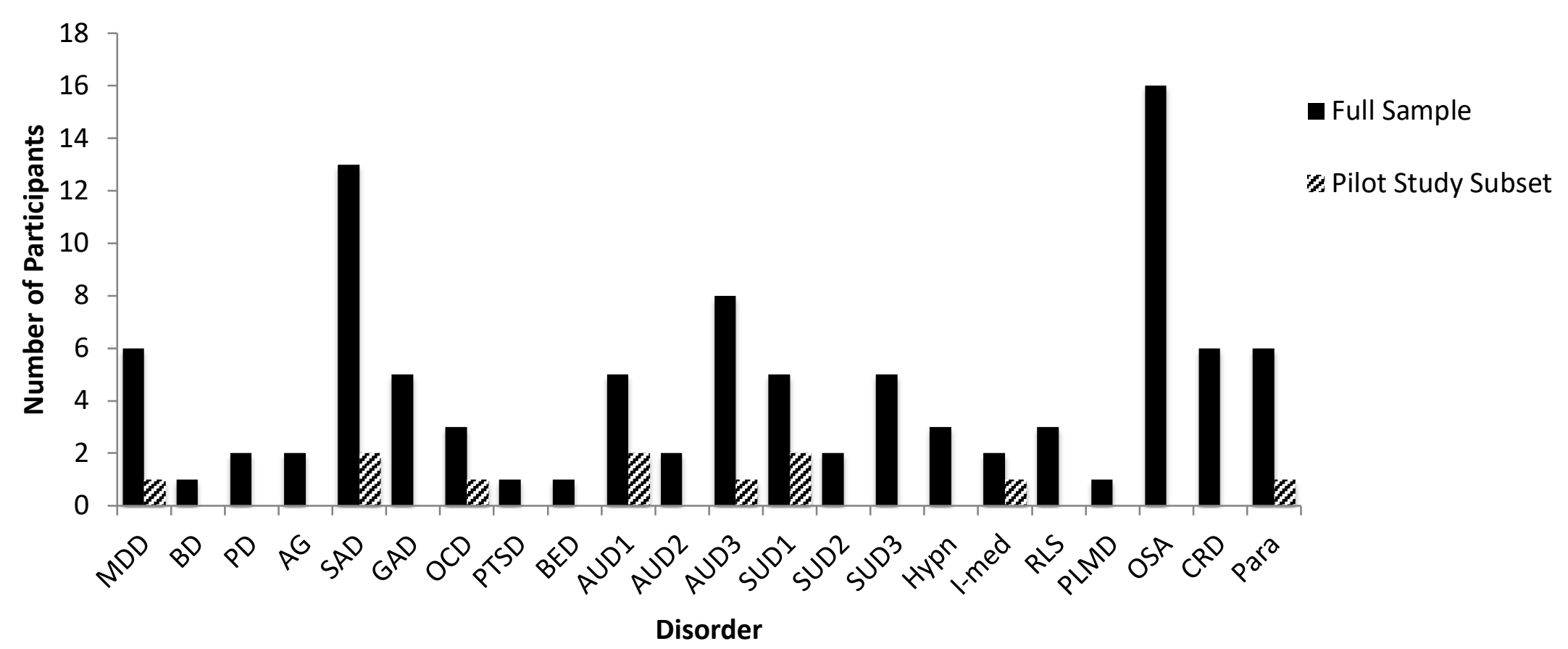

Figure 3. Frequency of comorbid psychiatric disorders at baseline. $\mathrm{AG}=$ agoraphobia; AUD1 $=$ alcohol use disorder, mild; AUD2 = alcohol use disorder, moderate; AUD3 = alcohol use disorder, severe; BED = binge eating disorder; $\mathrm{BD}=$ bipolar I disorder; $\mathrm{CRD}=$ circadian rhythm sleep-wake disorder; GAD = generalized anxiety disorder; Hypn = hypnotic dependence; I-med = insomnia due to a medical condition; $\mathrm{MDD}=$ major depressive disorder; $\mathrm{OCD}=$ obsessive-compulsive disorder; OSA = obstructive sleep apnea; Para = parasomnia; $\mathrm{PD}=$ panic disorder; PLMD = periodic limb movement disorder; PTSD = posttraumatic stress disorder; RLS $=$ restless legs syndrome; SAD = social anxiety disorder; SUD1 = substance use disorder, mild; SUD2 = substance use disorder, moderate;

SUD3 = substance use disorder, severe. Diagnoses based on the Mini International Neuropsychiatric Interview 7.0.1. and the Duke Structured Interview for Sleep Disorders. Participants listed as being diagnosed with OSA were screened positive, but not diagnosed, as polysomnography was not part of the study protocol. 
Insomnia covariates. Given the presence of ordinal data, and the small sample size, Spearman's rho $(\rho)$ was calculated to examine covariates of insomnia in the sample. Table 3 presents a correlation matrix of all variables with statistically significant correlations $(p<.05)$ with ISI, sleep efficiency, and total wake time, the main outcomes in the following CBT-I pilot study. While most sleep parameters collected using the CSD were correlated with ISI, sleep efficiency, and total wake time, as expected, the only significant correlations between psychological variables and indicators of insomnia were between the ISI and the following measures: GSES, $\rho=.51, p=<.001$, SE-S, $\rho=-.39, p=.007$, CES-D $, \rho=.34, p=.017$, DASSanxiety subscale, $\rho=.33, p=.021$, DASS-stress subscale, $\rho=.30, p=.040$, and SF-36 social functioning subscale, $\rho=-.34, p=.017$. Additionally, the SE-S was significantly correlated with both sleep efficiency, $\rho=.45, p=.002$, and total wake time, $\rho=-.45, p=.002$. No demographic or HIV-related clinical variables were significantly associated with ISI, sleep efficiency, or total wake time, including number of comorbid sleep and psychiatric disorders. While daytime sleepiness was not significantly correlated with indicators of insomnia in the current sample, the elevated mean ESS score, $8.1(S D=4.7)$, is worth noting.

Fatigue. Fatigue was not significantly associated with any indicators of insomnia in the current study. The mean FSS item score was $4.2(S D=1.2)$, and the mean FSS-7 item score, which has better psychometric properties among PWH (Lerdal et al., 2011), was $4.3(S D=1.4)$. For comparison, the mean FSS-7 item score among a large sample of PWH $(n=316)$ without insomnia or any other sleep disorder was 3.8 ( $S D=1.7$; Johansson, Kottorp, Lee, Gay, \& Lerdal, 2014). In the current study, fatigue was negatively associated with CD4 count, $\rho=-.44, p=.003$, time since HIV diagnosis, $\rho=-.40, p=.006$, and the following SF-36 subscales, which are scored with higher numbers representing better quality of life: role limitations due to physical 
Table 3

Spearman Correlations Between Indicators of Insomnia and Clinical Variables $(\mathrm{n}=48)$

\begin{tabular}{|c|c|c|c|c|c|c|c|c|c|c|c|c|c|c|c|}
\hline Variable & 1 & 2 & 3 & 4 & 5 & 6 & 7 & 8 & 9 & 10 & 11 & 12 & 13 & 14 & 15 \\
\hline 1. ISI & - & & & & & & & & & & & & & & \\
\hline 2. $\mathrm{SE}$ & $-.50^{* * *}$ & - & & & & & & & & & & & & & \\
\hline 3. TWT & $.46^{* *}$ & $-.95^{* * *}$ & - & & & & & & & & & & & & \\
\hline 4. $\mathrm{SOL}$ & $.44^{* *}$ & $-.42^{* *}$ & $.46^{* *}$ & - & & & & & & & & & & & \\
\hline 5. NWAK & $.34^{*}$ & $-.37^{* *}$ & $.37^{*}$ & .04 & - & & & & & & & & & & \\
\hline 6. WASO & -.03 & $-.45^{* *}$ & $.52^{* * *}$ & -.17 & $.40^{* *}$ & - & & & & & & & & & \\
\hline 7. TWASO & .16 & $-.45^{* *}$ & $.38^{* *}$ & -.06 & .09 & .01 & - & & & & & & & & \\
\hline 8. TST & $-.45^{* *}$ & $.79^{* * *}$ & $-.64^{* * *}$ & -.15 & $-.33^{*}$ & -.27 & $-.47^{* *}$ & - & & & & & & & \\
\hline 9. TIB & .01 & -.20 & $.36^{*}$ & $.34^{*}$ & .05 & .24 & -.03 & $.34^{*}$ & - & & & & & & \\
\hline 10. GSES & $.51^{* * *}$ & -.27 & .27 & $.36^{*}$ & .01 & -.02 & -.06 & -.24 & .07 & - & & & & & \\
\hline 11. SE-S & $-.39^{* *}$ & $.45^{* *}$ & $-.45^{* *}$ & $-.38^{*}$ & -.15 & -.17 & -.17 & $.36^{*}$ & -.12 & $-.62^{* * *}$ & - & & & & \\
\hline 12. DASS-a & $.33^{*}$ & -.26 & .23 & $.30^{*}$ & .17 & -.15 & .15 & -.18 & .12 & $.46^{* *}$ & $-.41^{* *}$ & - & & & \\
\hline 13. DASS-s & $.30^{*}$ & -.16 & .10 & .13 & .07 & -.28 & .14 & -.20 & .05 & $.46^{*}$ & $-.32^{*}$ & $.65^{* * *}$ & - & & \\
\hline 14. CES-D & $.34^{*}$ & -.22 & .23 & .20 & .05 & -.03 & .01 & -.14 & .21 & $.56^{* * *}$ & $-.49^{* *}$ & $.63^{* * *}$ & $.71^{* * *}$ & - & \\
\hline 15. SF-36-social & $-.34^{*}$ & .21 & -.24 & -.27 & -.12 & -.02 & .01 & .07 & -.22 & $-.30^{*}$ & $.33^{*}$ & $-.48^{* *}$ & $-.52^{* * *}$ & $-.76^{* * *}-$ & - \\
\hline
\end{tabular}

Note. CES-D = Centre for Epidemiological Studies in Depression Scale; DASS-a = Depression Anxiety Stress Scales-anxiety subscale; DASS-s = Depression Anxiety Stress Scales-stress subscale; GSES = Glasgow Sleep Effort Scale; ISI = Insomnia Severity Index; NWAK = number of awakenings; SE = sleep efficiency; SE-S = Self-Efficacy for Sleep Scale; SF-36-social = Medical Outcomes Study Short-Form Health Survey-social functioning subscale; SOL = sleep onset latency; TIB = time in bed; TST = total sleep time; TWASO = terminal wake after sleep onset; TWT = total wake time; WASO = wake after sleep onset. 
health, $\rho=-.72, p<.001$, role limitations due to emotional problems, $\rho=-.39, p=.007$, energy/fatigue, $\rho=-.49, p<.001$, emotional wellbeing, $\rho=-.31, p=.03$, social functioning, $\rho=$ $-.31, p=.033$, and general health, $\rho=-.29, p=.045$. Fatigue was positively associated with number of bothersome HIV-related symptoms, $\rho=.37, p=.009$, DASS depression subscale, $\rho=$ $.45, p=.001$, DASS anxiety subscale, $\rho=.36, p=.013$, DASS stress subscale, $\rho=.45, p=.007$, and CES-D, $\rho=.45, p=.001$.

\section{Association Between HIV- and Sleep-Related Variables}

With one notable exception, HIV-related variables were not significantly associated with any indicators of insomnia in the current study. However, they remain of clinical significance for PWH, and were associated with other sleep-related variables such as dysfunctional beliefs about sleep, pre-sleep arousal, sleep effort, self-efficacy for sleep, and number of nighttime awakenings. These include cART medication adherence, CD4 count, viral load, time since HIV diagnosis, and the presence of bothersome HIV-related symptoms. These associations are presented below.

cART medication regimen. All but four participants reported their cART medication regimen. Two reported not currently receiving cART, and another two did not provide data. Not all participants were on a single pill regimen. For data analysis, regimens were coded based on the single pill drug combination that was the primary component of a participant's cART regimen. The most frequently prescribed single cART pill was Triumeq (containing abacavir, lamivudine, and dolutegravir), reported by $27.1 \%(n=13)$ of participants as the sole, or primary component of, their cART regimen. The second most frequently prescribed single cART pill was Genvoya (containing cobicistat, evlitegravir, emtricitabine, and tenofovir), reported by $20.8 \%$ ( $n$ $=10)$ of participants. This was followed by Truvada (containing emtricitabine and tenofovir) 
plus an additional component, reported by $12.5 \%(n=6)$ of participants, Atripla (containing efavirenz, emtricitabine, and tenofovir), reported by $8.3 \%(n=4)$, Complera (containing rilpivirine, emtricitabine, and tenofovir), reported by $6.3 \%(n=3)$, and Trizivir (containing abacavir, lamivudine, and zidovudine), reported by $2.1 \%(n=1)$. Another $14.6 \%(n=7)$ of participants reported taking individually tailored combinations of different cART component medications. A Kruskal-Wallis test indicated no statistically significant difference in insomnia severity between groups of participants taking Triumeq, Genvoya, Truvada, Atripla, and the remaining regimens combined, $\chi^{2}(4)=5.50, p=.24$. Similarly, there were no statistically significant differences between groups in either sleep efficiency, $\chi^{2}(4)=2.73, p=.60$, or total wake time, $\chi^{2}(4)=2.95, p=.57$.

cART medication adherence. On the SRSI, a single item measure of cART medication adherence, $66.7 \%$ reported their adherence as excellent, and $27.1 \%$ and $6.3 \%$ reported their adherence as very good and good, respectively. SRSI score was negatively associated with number of awakenings, $\rho=-.29, p=.044$, and number of bothersome HIV-related symptoms, $\rho$ $=-.37, p=.01$. SRSI score was positively associated with the SF-36 subscale of role limitations due to emotional problems, $\rho=.47, p=.001$, on which higher scores represent higher quality of life. On the SMAQ, a composite measure of cART medication adherence, equal numbers of participants were identified as being adherent versus nonadherent. A point-biserial correlation was run to determine the relationship between cART adherence on the SMAQ and indicators of insomnia, and a statistically significant positive association was observed between adherence and sleep efficiency, $r_{\mathrm{pb}}=.31, p=.035$, such that patients who were nonadherent had greater sleep efficiency. The mean sleep efficiency for cART-adherent participants was $66.0 \%$, versus $75.5 \%$ for non-adherent participants. 
CD4 count. The mean CD4 count was 638 cells $/ \mu \mathrm{L}^{3}(S D=238)$, which is within the normal range of 500 cells $/ \mu \mathrm{L}^{3}$ to 1,600 cells $/ \mu \mathrm{L}^{3}$ for individuals with healthy immune functioning (Public Health Agency of Canada, 2016). The majority of participants (79.2\%) reported having a CD4 count $>350$ cells $/ \mu L^{3}$. CD4 count was positively associated with quality of life on the SF-36 general health subscale, $\rho=.40, p=.007$, in addition to the association mentioned above with fatigue.

HIV viral load. Almost all participants $(87.5 \%)$ reported having an undetectable viral load, and the remaining participants who knew their viral load all reported it to be $<100,000$ copies/mL, which is the cutoff above which cART treatment is recommended when CD4 count is $\geq 350$ cells $/ \mu L^{3}$ (Public Health Agency of Canada, 2016). Given the high proportion of participants with undetectable viral loads, the mean and standard deviation of this variable are not useful representations of the data.

Time since HIV diagnosis. Given the difficulty in knowing when, specifically, an individual has contracted HIV, time since HIV diagnosis was used as a proxy for time living with HIV. The mean time since HIV diagnosis was 12.5 years $(S D=9.3)$. As mentioned above, $54.2 \%$ of participants reported that they were diagnosed with HIV prior to experiencing insomnia, with the mean number of years between HIV diagnosis and insomnia onset among these participants being $11.3(S D=7.3)$. Another $12.5 \%$ of participants reported that insomnia onset coincided with their HIV diagnosis. Time since HIV diagnosis was positively associated with age, $\rho=.62, p=<.001$, SE-S, $\rho=.34, p=.025$, and the SF-36 energy/fatigue subscale, $\rho=$ $.34, p=.02$. It was negatively associated with GSES, $\rho=-.31, p=.035$, PSAS, $\rho=-.29, p=$ .048 , the SF-36 physical functioning subscale, $\rho=-.31, p=.037$, CES-D, $\rho=-.35, p=.019$, DASS depression subscale, $\rho=-.32, p=.031$, DASS anxiety subscale, $\rho=-.31, p=.038$, DASS 
stress subscale, $\rho=-.31, p=.039$, and FSS-7 as mentioned above. Given the association between time since HIV diagnosis and age, these associations were re-examined, controlling for age. After controlling for age, time since HIV diagnosis was negatively associated with GSES, $\rho=$ $.37, p=.016$, and positively associated with SE-S, $\rho=.33, p=.035$. Age was positively associated with two SF-36 subscales: energy/fatigue, $\rho=.45, p=.001$, and emotional wellbeing, $\rho=.36, p=.011$, and negatively associated with number of comorbid psychiatric disorders, $\rho=$ $.37, p=.009$, DASS depression subscale, $\rho=-.35, p=.016$, CES-D, $\rho=-.35, p=.014$, PSAS cognitive subscale, $\rho=-.29, p=.043$, and SF-36 physical functioning subscale, $\rho=-.38, p=$ .007 .

Bothersome HIV-related symptoms. On the HSI, the mean number of bothersome symptoms reported by participants was $10.3(S D=4.4)$. Number of bothersome symptoms was positively associated with number of comorbid psychiatric disorders, $\rho=.31, p=.030$, DBAS$16, \rho=.41, p=.004$, GSES, $\rho=.31, p=.032$, PSAS, $\rho=.49, p<.001$, DASS depression subscale, $\rho=.45, p=.001$, DASS anxiety subscale, $\rho=.63, p<.001$, DASS stress subscale, $\rho=$ $.42, p=.003$, and CES-D, $\rho=.52, p<.001$, as well as FSS-7 as mentioned above. Number of bothersome symptoms was negatively associated with cART adherence as mentioned above, and quality of life on the following SF-36 subscales: role limitations due to physical health, $\rho=-.32$, $p=.028$, role limitations due to emotional problems, $\rho=-.51, p<.001$, emotional wellbeing, $\rho=$ $-.50, p<.001$, social functioning, $\rho=-.40, p=.005$, and pain, $\rho=-.49, p=.001$.

Bothersome symptoms are those that participants reported "bother me a little", "bother me", or "bother me a lot". According to this operationalization, the most frequently reported bothersome symptoms were fatigue/loss of energy (95.9\%), difficulty falling/staying asleep (95.8\%), muscle aches/joint pain (66.7\%), feeling nervous/anxious (66.7\%), bloating/pain/gas in 
stomach (64.7\%), feeling sad/down/depressed (62.5\%), changes in body appearance (62.4\%), problems having sex, including loss of interest/satisfaction (60.5\%), trouble remembering $(54.2 \%)$, and skin problems such as rash/dryness/itching (50\%). Using more stringent criteria for determining bothersome symptoms, including symptoms that participants report "bother me" or "bother me a lot", the most frequently reported symptoms were difficulty falling/staying asleep (85.4\%), fatigue/loss of energy (64.6\%), muscle aches/joint pain (47.9\%), feeling nervous/anxious (41.7\%), changes in body appearance (41.6\%), feeling sad/down/depressed (37.5\%), problems having sex, including loss of interest/satisfaction (35.5\%), trouble remembering (35.4\%), bloating/pain/gas in stomach $(33.4 \%)$, and skin problems such as rash/dryness/itching (33.3\%).

\section{Summary of Results Exploring Features of Insomnia among PWH}

Participants experienced chronic insomnia, and only a minority of participants reported that insomnia onset coincided with HIV diagnosis. The majority of participants reported that HIV diagnosis preceded insomnia onset, but the mean time between HIV diagnosis and insomnia onset was over ten years, suggesting that, not surprisingly, insomnia among PWH is not directly associated with HIV infection or communication of diagnosis, and that other factors play an important role. The mean insomnia severity score was in the range indicative of moderate insomnia severity. Sleep maintenance was more of a problem than sleep onset, with $75 \%$ of participants classified as poor sleepers based on wakefulness after sleep onset, and $58.3 \%$ based on sleep onset latency. Mean sleep efficiency was $70 \%$, well below the cutoff of $85 \%$ used to differentiate individuals with insomnia from good sleepers, and mean total wake time was over two hours. Relevant targets for CBT-I were identified in this sample, including daytime napping, 
extended time in bed, engaging in non-sleep activities in the bedroom, and experiencing worry, anxiety, and/or physical tension when attempting to sleep.

Comorbidity was high in this sample, with $54.2 \%$ being diagnosed with at least one nonsleep psychiatric disorder, $54.2 \%$ with at least one sleep disorder in addition to insomnia, and $72.9 \%$ with at least one of any psychiatric disorder in addition to insomnia (including sleep disorders). The most common comorbid conditions were OSA (33.3\%), social anxiety disorder (27.1\%), and alcohol use disorder, severe (16.7\%). Insomnia severity was positively associated with several psychological variables, including sleep effort, depression, anxiety, and stress. Insomnia severity was negatively associated with quality of life (social functioning subscale) and self-efficacy for sleep. Self-efficacy for sleep was positively associated with sleep efficiency and negatively associated with total wake time. Fatigue was not associated with indicators of insomnia, but was positively associated with depression, anxiety, stress, and number of bothersome HIV-related symptoms. It was negatively associated with CD4 count, time since HIV diagnosis, and several domains of quality of life, including role limitations due to physical health, role limitations due to emotional problems, energy/fatigue, emotional wellbeing, social functioning, and general health.

HIV-related variables were not associated, for the most part, with any indicators of insomnia. Mean CD4 count was within the normal range for adults with healthy immune functioning, and viral load was reported as undetectable for the majority of participants. CD4 count was negatively associated with fatigue and positively associated with quality of life (general health subscale). Almost all participants reported cART adherence as either excellent or very good, however, on a composite dichotomous measure of adherence, only half were identified as adherent. Greater adherence to cART was associated with poorer sleep efficiency, 
however, this finding was not consistent across both measures of cART adherence. Furthermore, cART regimen was not associated with indicators of insomnia. Triumeq and Genvoya were the most commonly prescribed cART regimens, accounting for almost $50 \%$ of regimens. The mean time since HIV diagnosis was 12.5 years, and after controlling for age, this variable was associated with greater self-efficacy for sleep and lower sleep effort. Finally, number of bothersome HIV-related symptoms was positively associated with number of comorbid psychiatric disorders, dysfunctional beliefs about sleep, sleep effort, pre-sleep arousal, depression, anxiety, stress and fatigue. It was negatively associated with cART adherence, and several domains of quality of life, including role limitations due to physical health, role limitations due to emotional problems, emotional wellbeing, social functioning, and pain.

\section{CBT-I Pilot Study}

Demographic data for pilot study participants are presented in Table 1. Pre- and posttreatment data for sleep, HIV, and non-sleep psychological variables are presented in Table 4 along with $M, S D$, and Cohen's $d$ representing baseline to posttreatment effect size.

Intervention effects. With regard to symptom change, it was hypothesized that from baseline to posttreatment, participants would experience 1) significant reduction in subjective insomnia symptom severity as measured by the ISI, 2) improvement in sleep efficiency, with a target sleep efficiency between $85 \%$ and $90 \%$, and 3) reduction in total wake time, 4) reduction in viral load for participants with a detectable viral load at baseline, 5) increase in CD4 count, and 6) improved cART medication adherence. To examine Hypotheses 1, 2, and 3, change over time on primary outcomes of ISI, sleep efficiency, and total wake time were examined using visual inspection.

Hypothesis 1: Insomnia severity. Mean ISI score reduced from $19.7(S D=2.7)$ at 
Table 4

Clinical Characteristics of Pilot Study Participants Pre- and Post-CBT-I (n = 10)

\begin{tabular}{|c|c|c|c|c|c|}
\hline \multirow[b]{2}{*}{ Variable } & \multicolumn{2}{|c|}{ Pre-CBT-I } & \multicolumn{2}{|c|}{ Post-CBT-I } & \multirow[b]{2}{*}{$d$} \\
\hline & $\%(n)$ & $M(S D)$ & $\%(n)$ & $M(S D)$ & \\
\hline \multicolumn{6}{|l|}{ Sleep-related variables } \\
\hline \multicolumn{6}{|c|}{ Consensus Sleep Diary (2-week period) } \\
\hline $\mathrm{SOL}(\mathrm{min})$ & & $40.1(34.6)$ & & $15.0(7.8)$ & 0.72 \\
\hline NWAK & & $2.8(1.52)$ & & $1.3(1.2)$ & 0.99 \\
\hline WASO (min) & & $81.3(77.0)$ & & $16.4(16.7)$ & 0.85 \\
\hline TWASO (min) & & $61.6(35.6)$ & & $12.6(11.8)$ & 1.39 \\
\hline TIB (min) & & $530.5(58.5)$ & & $420.4(55.4)$ & 1.88 \\
\hline TST (min) & & $318.2(78.8)$ & & $360.0(55.1)$ & -0.53 \\
\hline TWT (min) & & $183.1(87.6)$ & & $44.0(25.7)$ & 1.59 \\
\hline $\mathrm{SE}$ & & $0.65(0.16)$ & & $0.89(0.06)$ & -1.50 \\
\hline Daytime napping (min) & & $38.6(41.4)$ & & $8.5(8.5)$ & 0.72 \\
\hline ISI & & $19.7(2.7)$ & & $7.1(5.3)$ & 4.67 \\
\hline ESS & & $8.3(4.1)$ & & $-{ }^{\mathrm{a}}$ & \\
\hline DBAS-16 & & $5.9(1.7)$ & & $4.3(1.9)$ & 0.94 \\
\hline GSES & & $8.5(2.9)$ & & $3.6(2.9)$ & 1.69 \\
\hline PSAS-13 & & $32.0(9.3)$ & & $29.1(8.4)$ & 0.31 \\
\hline cognitive subscale & & $16.2(4.5)$ & & $13.8(3.6)$ & 0.53 \\
\hline somatic subscale & & $15.7(6.7)$ & & $15.3(6.4)$ & 0.06 \\
\hline SE-S & & $43.1(19.2)$ & & $66.4(15.2)$ & -1.21 \\
\hline FSS-7 & & $5.0(1.1)$ & & $4.1(1.9)$ & 0.82 \\
\hline \multicolumn{6}{|l|}{ HIV-related variables } \\
\hline CD4 count & & $620.9(253.9)$ & & $687.2(303.2)$ & -0.26 \\
\hline Over $350 / \mu \mathrm{L}^{3}$ & $70(7)$ & & $70(7)$ & & \\
\hline $200-350 / \mu \mathrm{L}^{3}$ & $10(1)$ & & $20(2)$ & & \\
\hline$<200 / \mu \mathrm{L}^{3}$ & - & & $10(1)$ & & \\
\hline Can't remember/not told & $20(2)$ & & - & & \\
\hline Viral load & & $4.0(12.6)^{b}$ & & $4.5(14.2)^{b}$ & -0.04 \\
\hline Undetectable & $90(9)$ & & $100(10)$ & & \\
\hline$<100,000$ copies $/ \mathrm{mL}$ & - & & - & & \\
\hline Waiting for results & $10(1)$ & & - & & \\
\hline \multicolumn{6}{|l|}{ SRSI (cART adherence) } \\
\hline Excellent & $70(7)$ & & $100(10)$ & & \\
\hline Very good & $20(2)$ & & - & & \\
\hline Good & $10(1)$ & & - & & \\
\hline SMAQ: Adherent to cART & $40(4)$ & & $30(3)^{\mathrm{c}}$ & & \\
\hline
\end{tabular}

(continued) 


\begin{tabular}{|c|c|c|c|c|c|}
\hline \multirow[b]{2}{*}{ Variable } & \multicolumn{2}{|c|}{ Pre-CBT-I } & \multicolumn{2}{|c|}{ Post-CBT-I } & \multirow[b]{2}{*}{$d$} \\
\hline & $\%(n)$ & $M(S D)$ & $\%(n)$ & $M(S D)$ & \\
\hline \multicolumn{6}{|l|}{ HIV-related variables (continued) } \\
\hline HSI-bothersome & & $10.2(3.5)$ & & $8.4(3.2)$ & 0.51 \\
\hline \multicolumn{6}{|l|}{ SF-36 } \\
\hline Physical functioning & & $80.0(21.6)$ & & $80.5(18.5)$ & -0.02 \\
\hline Role limitations-physical & & $32.5(44.2)$ & & $47.5(46.3)$ & -0.34 \\
\hline Role limitations-emotional & & $46.7(50.2)$ & & $53.3(50.2)$ & -0.13 \\
\hline Energy/fatigue & & $36.5(20.7)$ & & $45.0(15.8)$ & -0.41 \\
\hline Emotional wellbeing & & $69.2(19.4)$ & & $72.0(17.7)$ & -0.14 \\
\hline Social functioning & & $53.8(22.1)$ & & $57.5(24.4)$ & -0.17 \\
\hline Pain & & $67.2(28.2)$ & & $61.0(25.4)$ & 0.22 \\
\hline General health & & $53.5(22.5)$ & & $55.5(25.1)$ & -0.09 \\
\hline \multicolumn{6}{|l|}{ Psychological variables } \\
\hline DASS-21 & & $32.2(16.6)$ & & $29.3(26.4)$ & 0.17 \\
\hline Depression & & $9.2(5.4)$ & & $10.0(10.8)$ & -0.15 \\
\hline Anxiety & & $8.4(6.9)$ & & $8.8(9.0)$ & -0.06 \\
\hline Stress & & $14.8(8.6)$ & & $10.6(8.4)$ & 0.49 \\
\hline CES-D & & $19.8(9.6)$ & & $15.4(12.0)$ & 0.46 \\
\hline AUDIT & & $6.4(4.2)$ & & $-^{\mathrm{a}}$ & \\
\hline DUDIT & & $2.6(3.4)$ & & $-^{\mathrm{a}}$ & \\
\hline \multicolumn{6}{|c|}{$\begin{array}{l}\text { Note. Participants in the pilot study subset }(n=10) \text { are also included in the full sample }(N=48) \text {. } \\
\text { AUDIT = Alcohol Use Disorders Identification Test; CES-D = Center for Epidemiological } \\
\text { Studies in Depression Scale; } d=\text { Cohen's } d \text { effect size based on } S D \text { of pretreatment scores; } \\
\text { DASS-21 = Depression, Anxiety, Stress Scales; DBAS-16 = Dysfunctional Beliefs About Sleep } \\
\text { Scale; DUDIT = Drug Use Disorders Identification Test; ESS = Epworth Sleepiness Scale; FSS- } \\
7 \text { = Fatigue Severity Scale; GSES = Glasgow Sleep Effort Scale; HSI-bothersome = number of } \\
\text { bothersome symptoms reported on HIV Symptom Index; ISI = Insomnia Severity Index; NWAK } \\
\text { = number of awakenings; PSAS-13 = Pre-Sleep Arousal Scale; SE = sleep efficiency; SE-S = } \\
\text { Self-Efficacy for Sleep Scale; SF-36 = Medical Outcomes Study Short-Form Health Survey; } \\
\text { SMAQ = Simplified Medication Adherence Questionnaire; SOL = sleep onset latency; SRSI = } \\
\text { Self-Rating Scale Item; TIB = time in bed; TST = total sleep time; TWASO = terminal wake } \\
\text { after sleep onset; TWT = total wake time; WASO = wake after sleep onset. } \\
\text { aVariable not measured posttreatment as it was used to assess exclusion criteria. } \\
\text { bViral load } M \text {, SD should be interpreted with caution given high frequency of reported } \\
\text { undetectable viral loads. } \\
\text { 'Missing data prevented calculation of composite score for } 2 \text { participants. }\end{array}$} \\
\hline
\end{tabular}


baseline to $7.1(S D=5.3)$ at posttreatment. These scores are indicative of moderate and no insomnia, respectively. Cohen's $d$, based on the $S D$ of baseline ISI scores was $d=4.67$, indicating a large effect of treatment on insomnia severity. Given that not all participants ended up receiving exactly four therapy sessions (discussed below), the number of data points analyzed using visual inspection ranged from six to nine per participant. All participants had at least three data points to serve as baseline comparison prior to receiving CBT-I. With regard to magnitude of change, visual inspection of ISI scores for each participant relative to their respective baseline period $M$ and $\pm 2 S D$ revealed that for eight participants (P1, P2, P3, P5, P7, P8, P9, P10), ISI scores for at least the final two time points, which corresponded with the final session and posttreatment assessment, were $>2 S D$ below each participant's own baseline period mean. For one additional participant, P4, ISI scores for two non-consecutive time points out of the final three time points were $>2 S D$ below the baseline period mean, and the remaining score was exactly $2 S D$ below the mean. Only one participant, P6, had ISI scores that remained within $2 S D$ of the baseline mean throughout treatment. With regard to latency of change, a sustained mean ISI score $>2 S D$ below the baseline period mean was observed after just one session for four participants, after two sessions for one participant, after three sessions for three participants, and not at all for two participants. Mean ISI score for the sample decreased $>2 S D$ below the baseline period mean after just one session (Figure 4).

With regard to trend, visual inspection of celeration lines representing slope of ISI scores in the baseline versus intervention periods revealed that for all but one participant (P2), a steeper downward slope was visually detectable in the intervention period relative to the baseline period. Data from participant P2 demonstrated a slightly less steep slope in the intervention period relative to baseline period; however, reliable magnitude of change was identified for P2 using the 


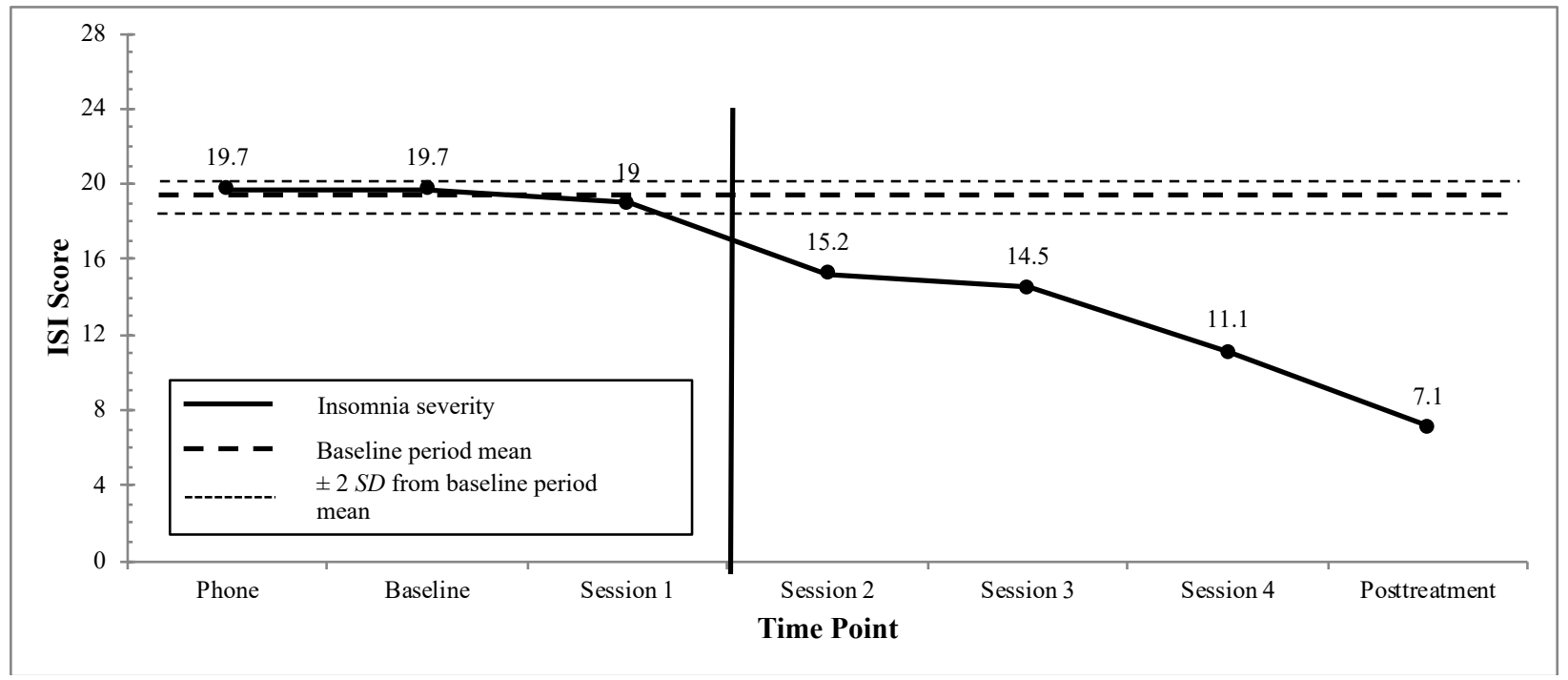

Figure 4a. Mean Insomnia Severity Index scores with baseline period mean $\pm 2 S D$ plotted $(n=$ 10).

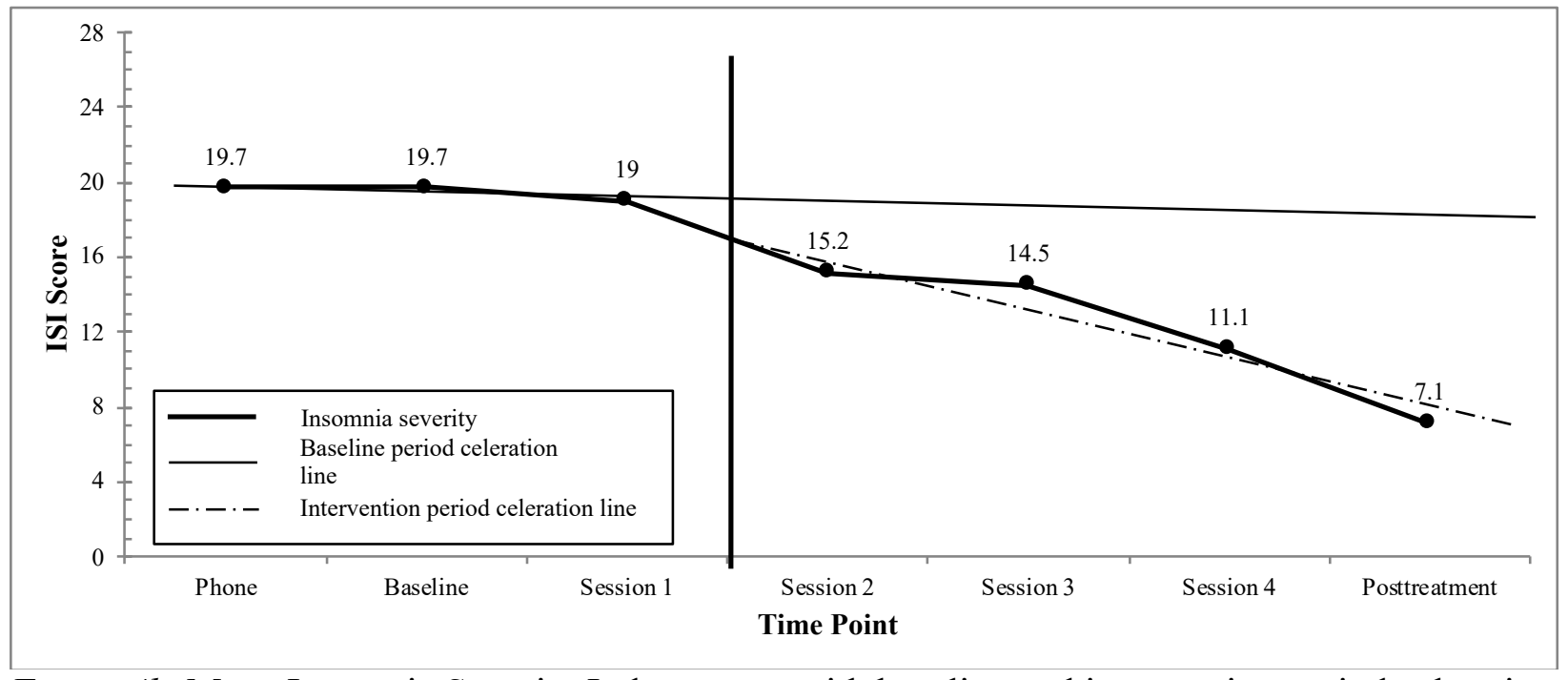

Figure 4b. Mean Insomnia Severity Index scores with baseline and intervention period celeration lines plotted. Celeration lines indicate slope of change during each period, with the baseline celeration line extended into the intervention period for comparison $(n=10)$. 
$M / S D$ visual aid. Reliable change in insomnia severity based on at least one indicator, either magnitude or slope, was identified for all participants; however, reliable change on both indicators of magnitude and slope was identified for only seven participants. Using a slightly less conservative criterion of $\geq 2 S D$ (i.e., including $2 S D$ as an indicator of change) to identify reliable change, reliable change in insomnia severity on both indicators was observed among eight participants. Reliable change was also observed with regard to change in slope of mean ISI score from baseline to intervention period. Figure 4 presents the sample mean ISI score at each time point, accompanied by visual aids, and change in ISI for each participant is presented in Figures 5-14.

With regard to clinically significant improvement, it has been proposed that a reduction in ISI total score $>7$ and $>8$ points is considered indicative of moderate and marked clinical improvement, respectively (Morin, Belleville et al., 2011). According to these criteria, two participants improved moderately, and seven markedly. Clinically significant improvement was observed in all but one participant, P6. Half of participants met criteria for insomnia remission at posttreatment, ISI $<8$ (Carney et al., 2017).

Although the study was not designed to examine statistical significance of change over time, exploratory analyses were conducted to examine change in ISI, sleep efficiency, total wake time, and CD4 count from pre- to posttreatment, using a series of paired samples $t$-tests applying bias-corrected bootstrapping to account for non-normally distributed data. For the bootstrapping analyses, 5000 samples were run based on a $95 \%$ confidence interval. ISI score significantly reduced from baseline $(M=19.7, S D=2.7)$ to posttreatment $(M=7.1, S D=5.3), t(9)=6.54, p<$ .001 , bias-corrected $95 \%$ CI $[9.17,16.10]$. The first hypothesis, that participants would experience a significant reduction in subjective insomnia symptom severity as measured by the 


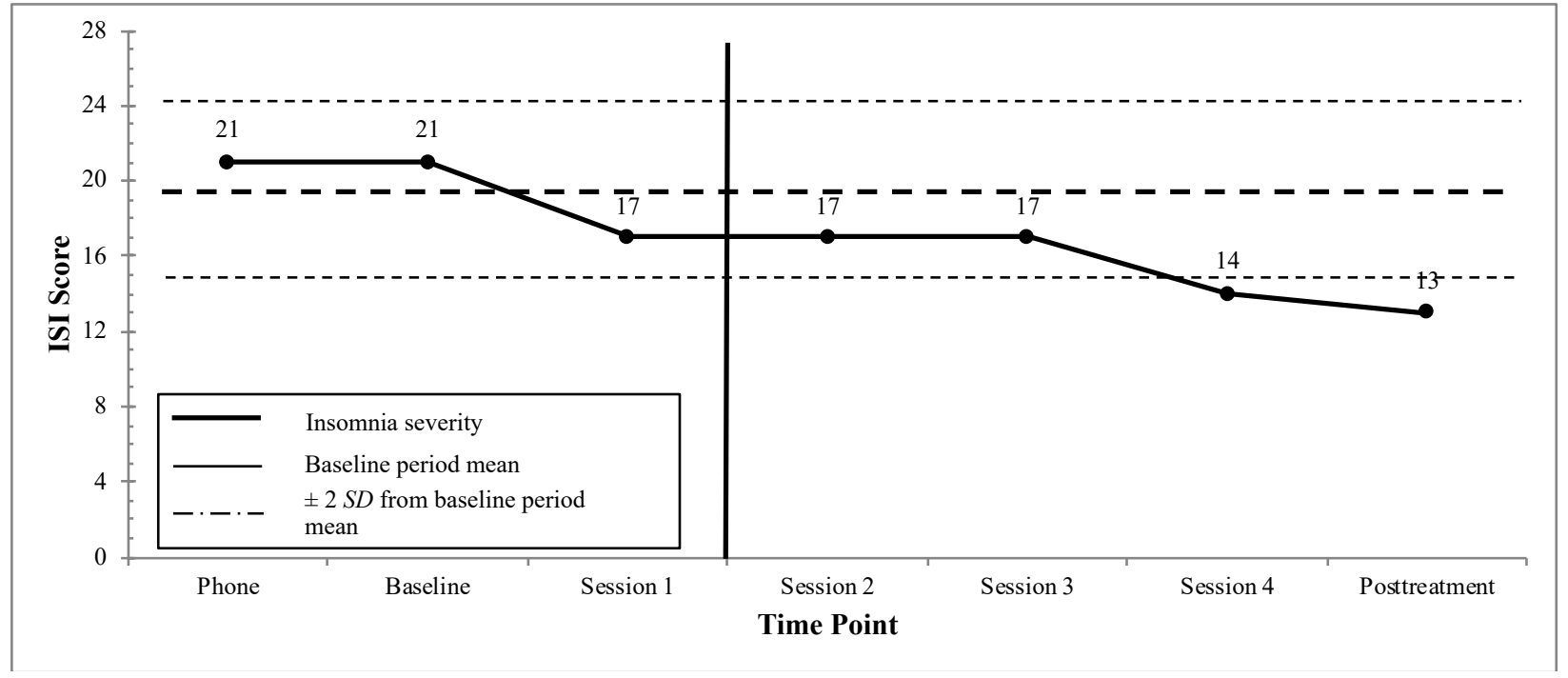

Figure 5a. Participant P1 Insomnia Severity Index scores with baseline period mean $\pm 2 S D$ plotted.

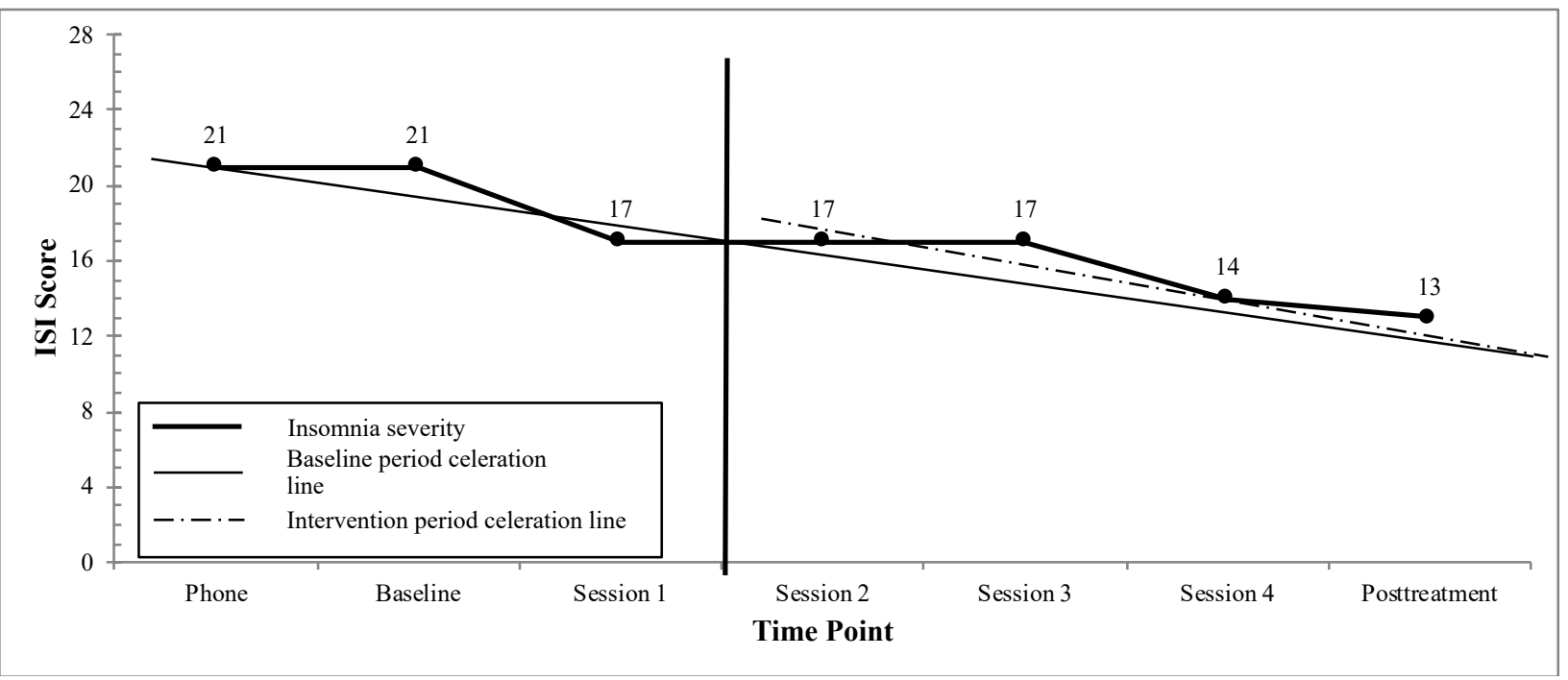

Figure 5b. Participant P1 Insomnia Severity Index scores with baseline and intervention period celeration lines plotted. Celeration lines indicate slope of change during each period, with the baseline celeration line extended into the intervention period for comparison. 


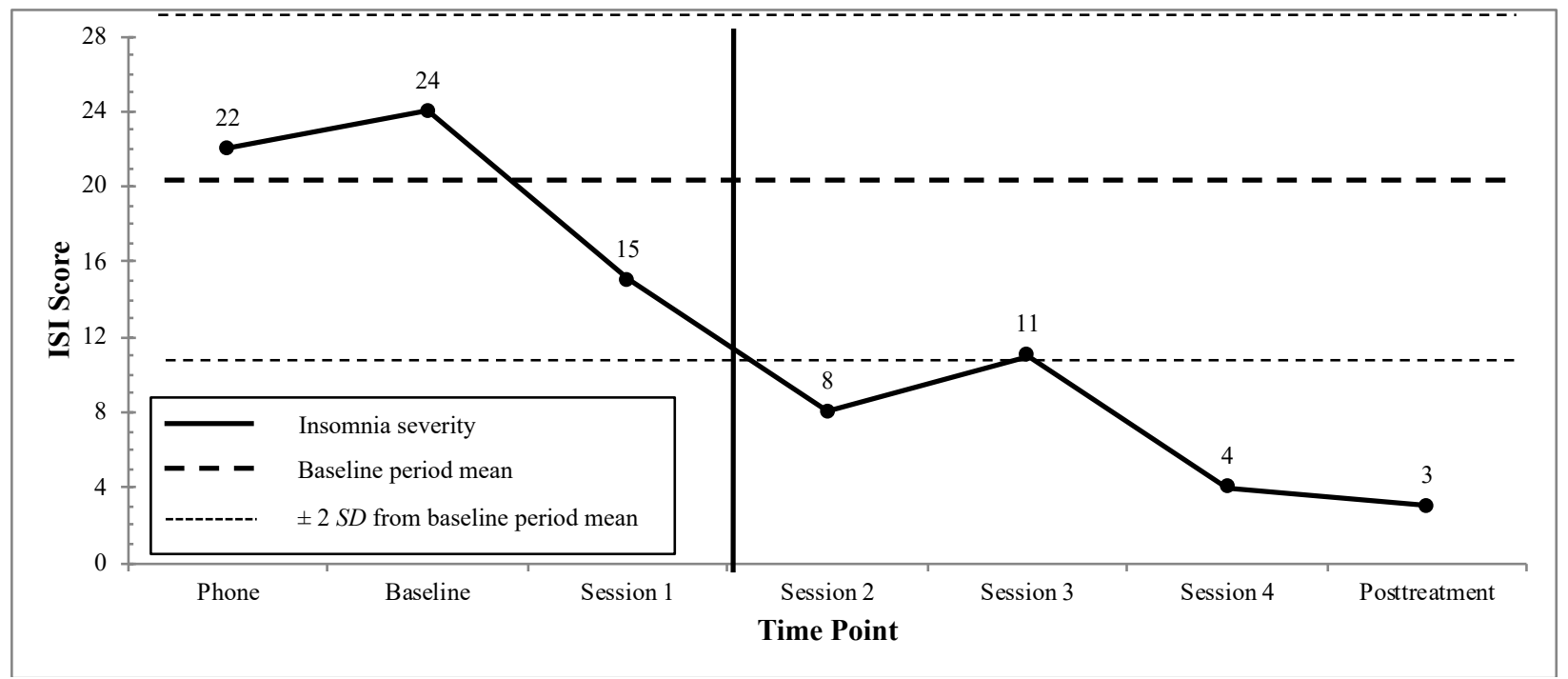

Figure 6a. Participant P2 Insomnia Severity Index scores with baseline period mean $\pm 2 S D$ plotted.

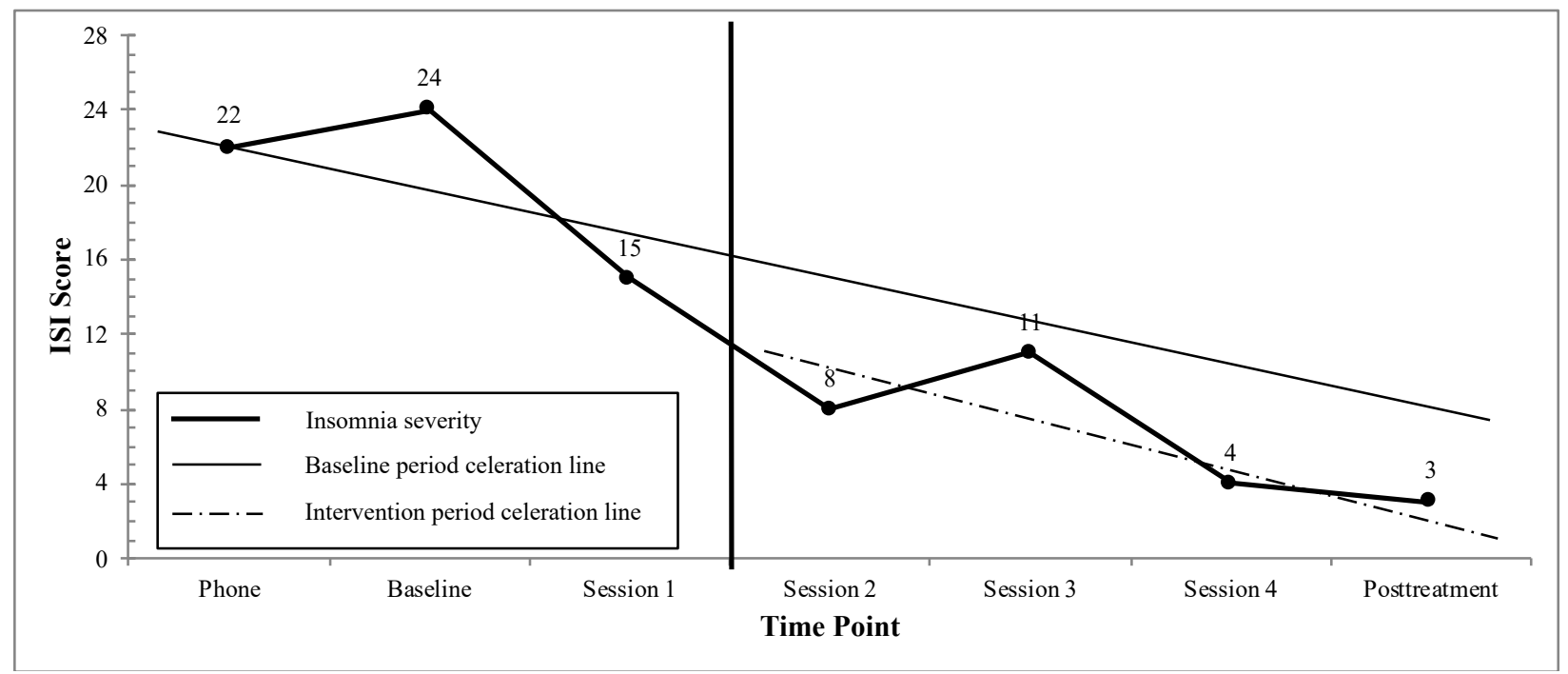

Figure 6b. Participant P2 Insomnia Severity Index scores with baseline and intervention period celeration lines plotted. Celeration lines indicate slope of change during each period, with the baseline celeration line extended into the intervention period for comparison. 


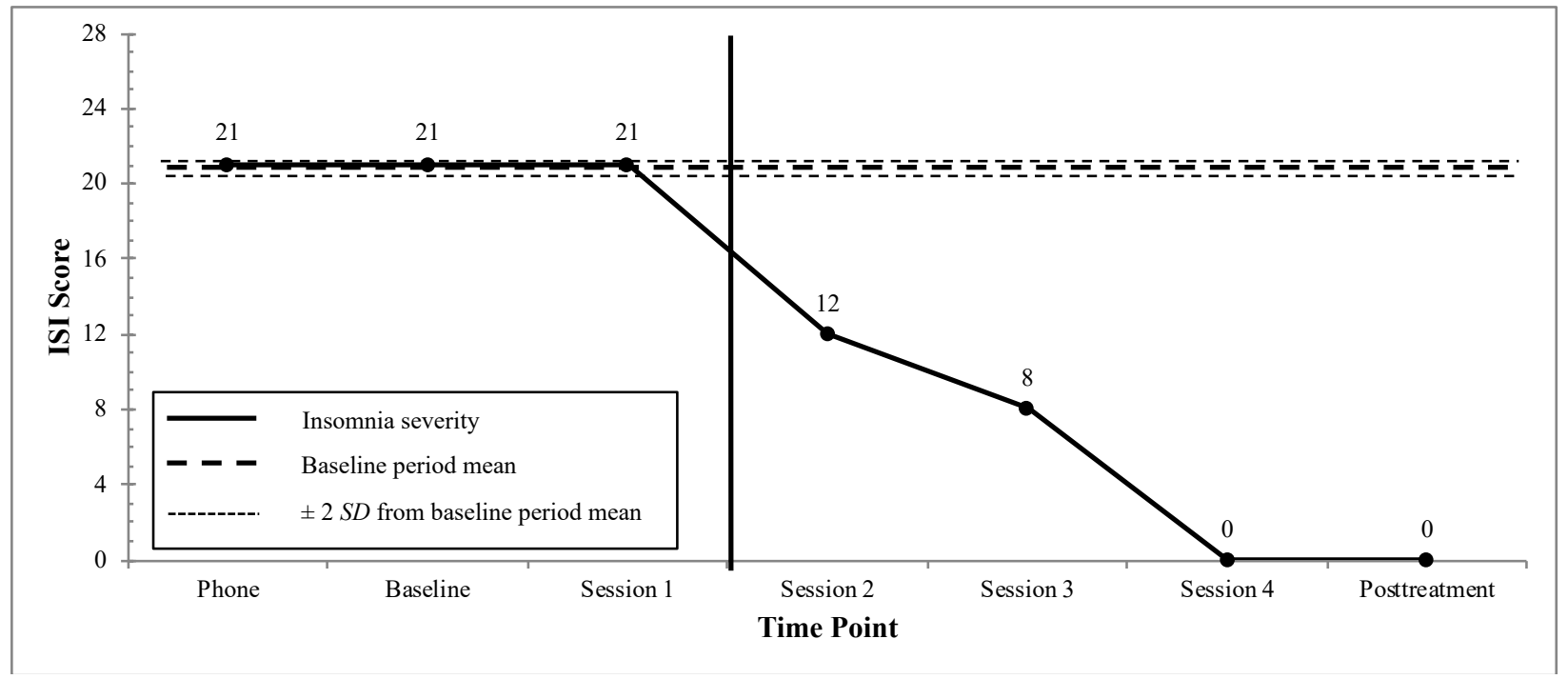

Figure 7a. Participant P3 Insomnia Severity Index scores with baseline period mean $\pm 2 S D$ plotted.

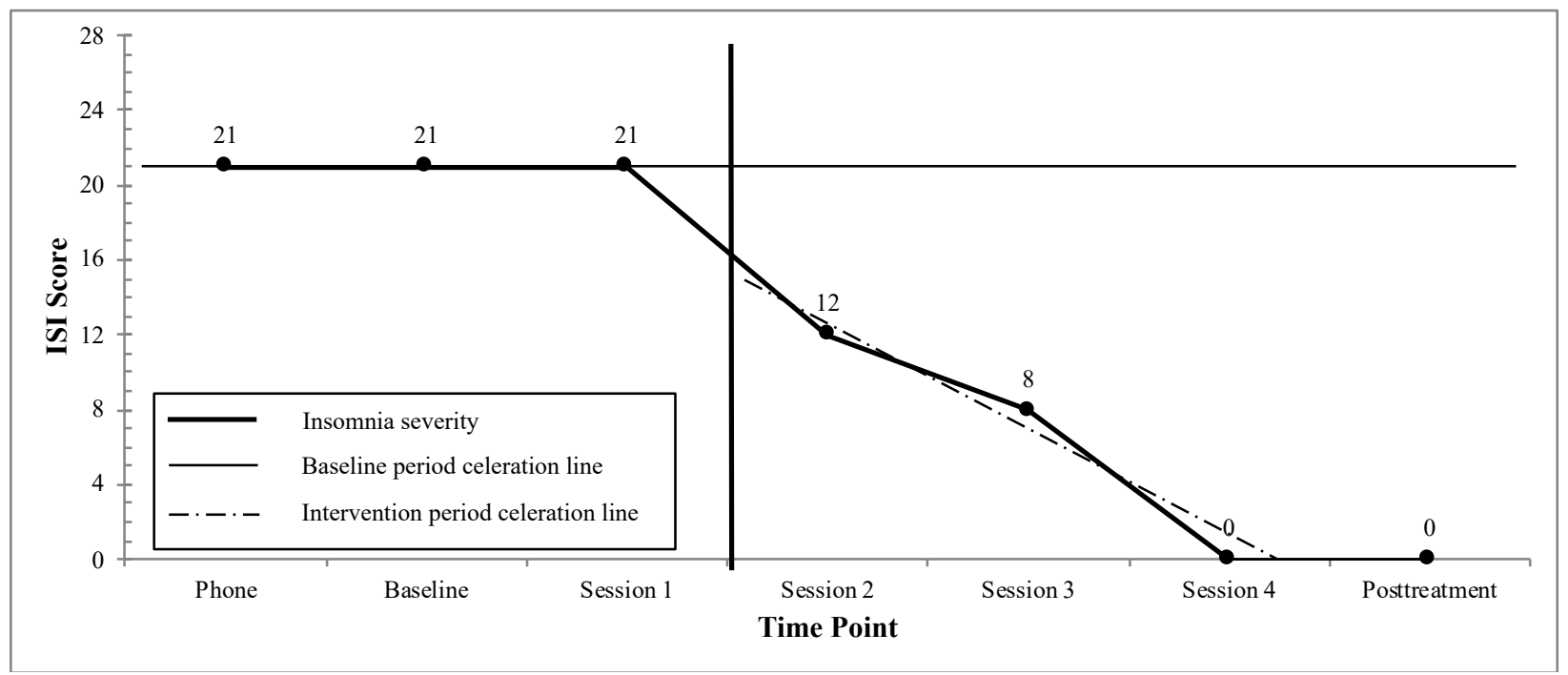

Figure 7b. Participant P3 Insomnia Severity Index scores with baseline and intervention period celeration lines plotted. Celeration lines indicate slope of change during each period, with the baseline celeration line extended into the intervention period for comparison. 


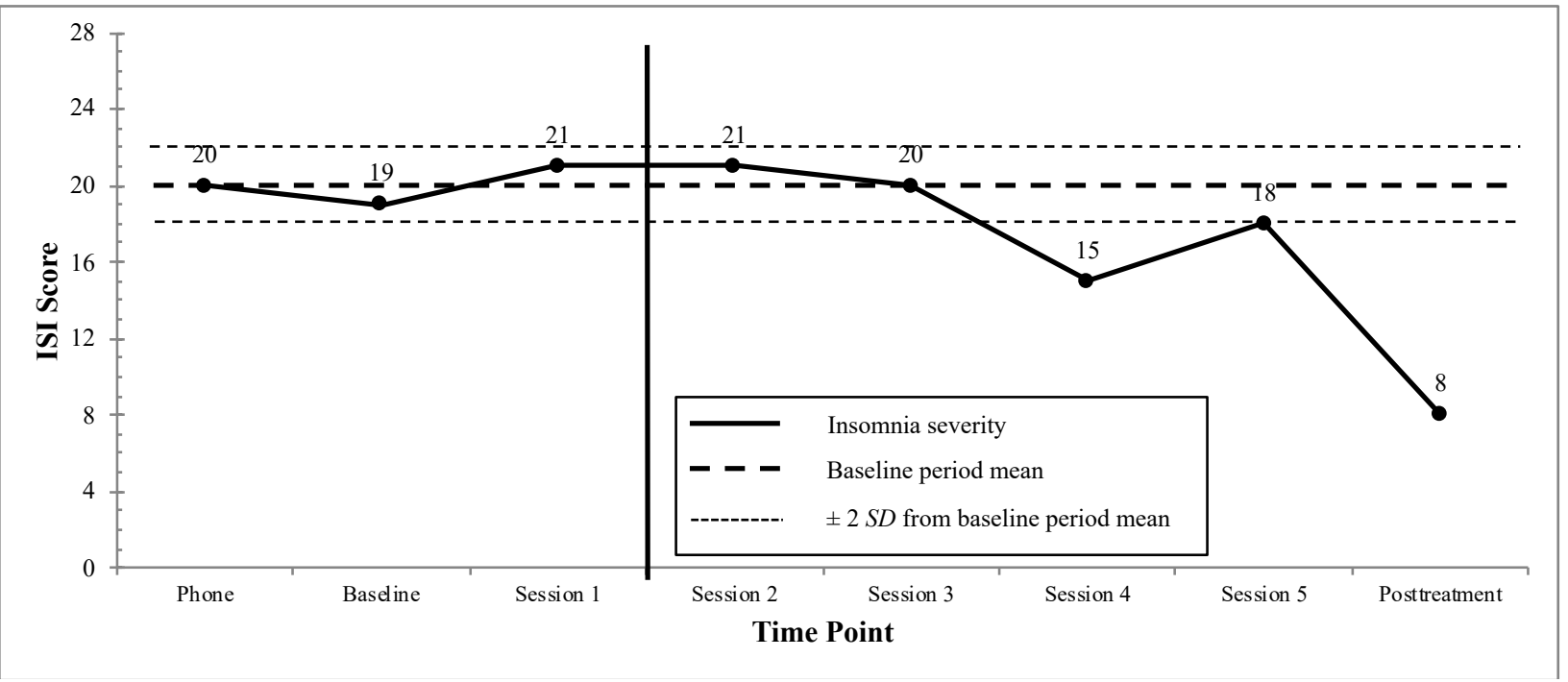

Figure $8 a$. Participant P4 Insomnia Severity Index scores with baseline period mean $\pm 2 S D$ plotted.

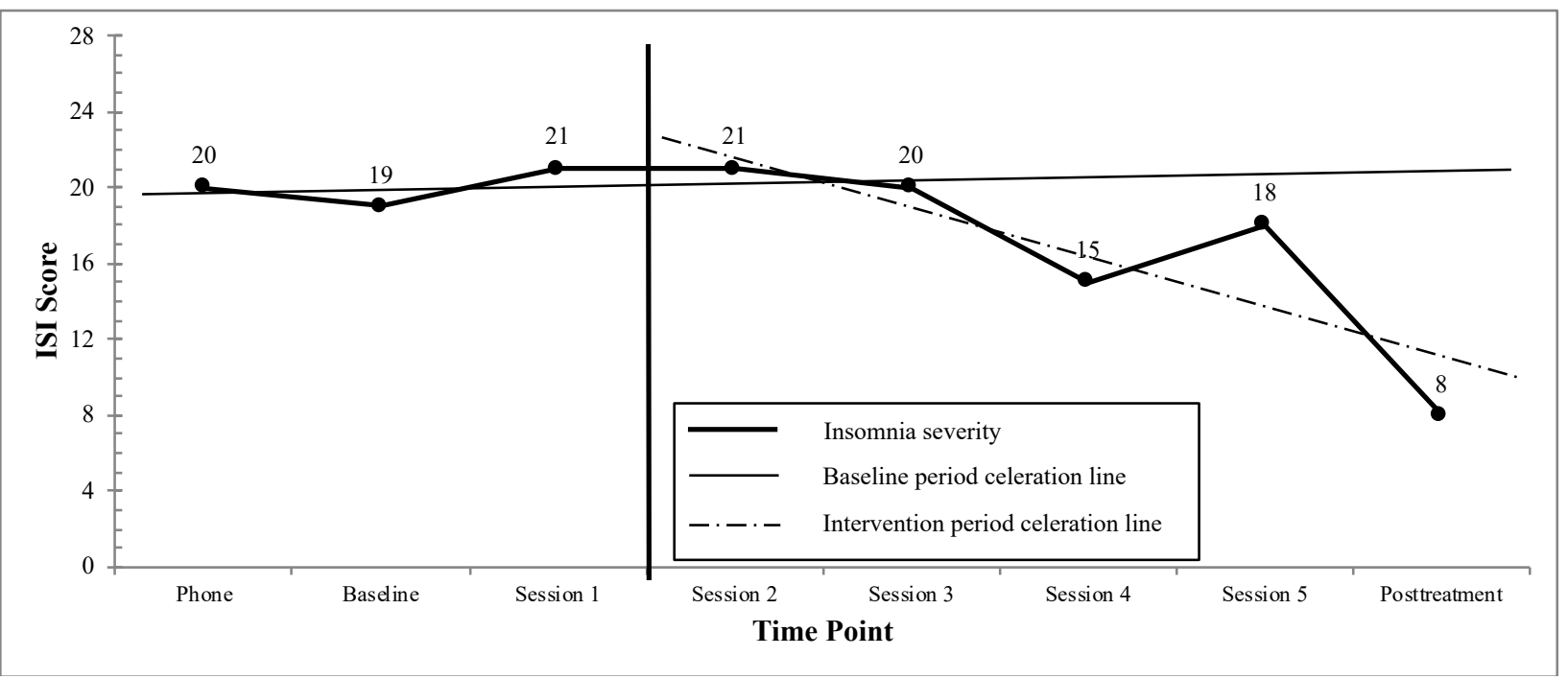

Figure 8b. Participant P4 Insomnia Severity Index scores with baseline and intervention period celeration lines plotted. Celeration lines indicate slope of change during each period, with the baseline celeration line extended into the intervention period for comparison. 


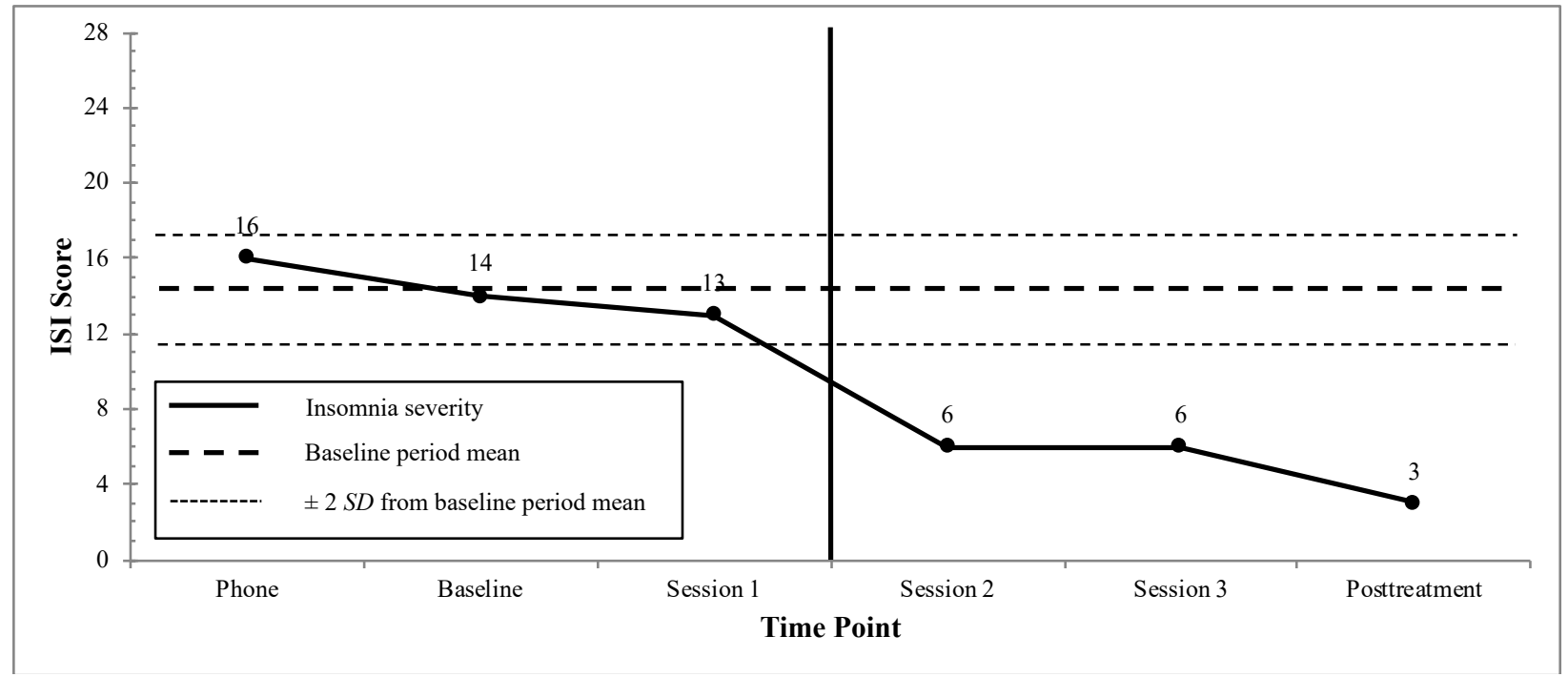

Figure 9a. Participant P5 Insomnia Severity Index scores with baseline period mean $\pm 2 S D$ plotted.

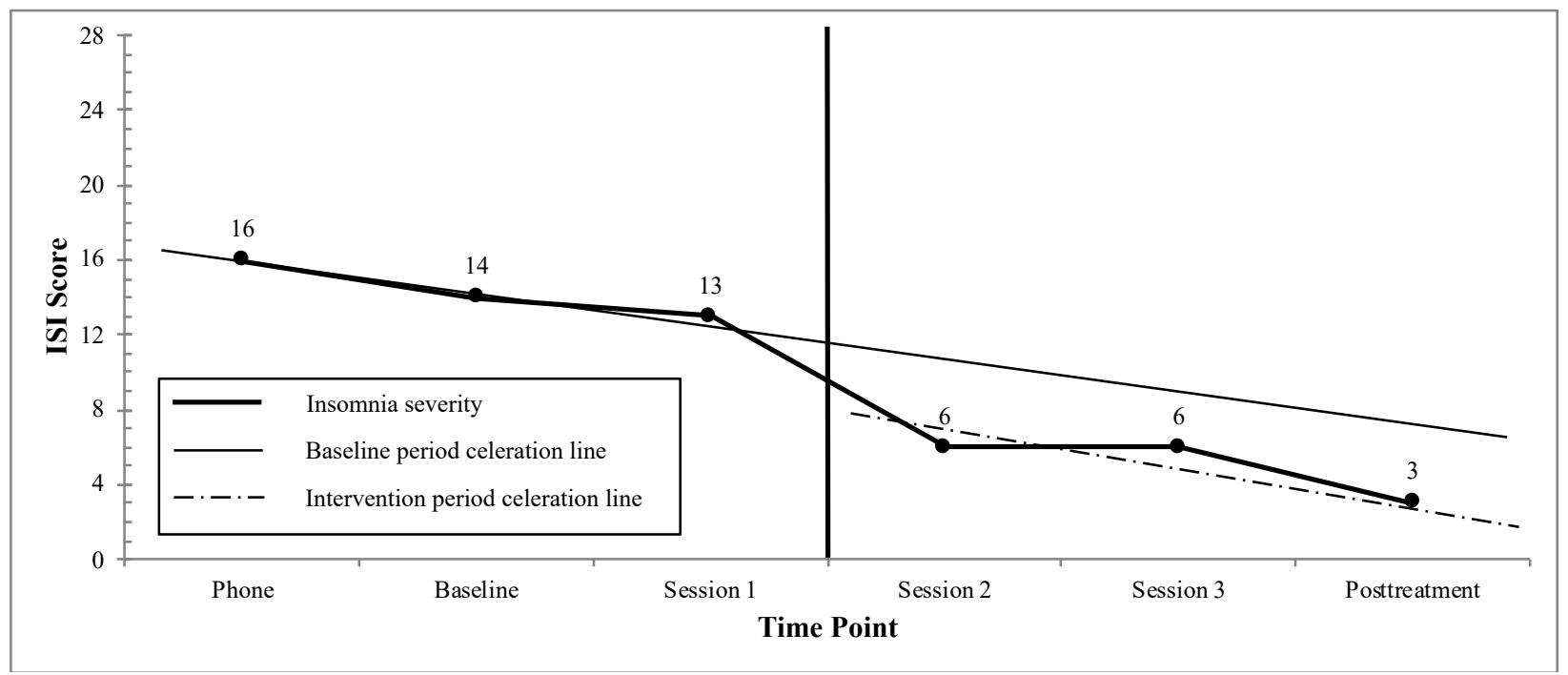

Figure 9b. Participant P5 Insomnia Severity Index scores with baseline and intervention period celeration lines plotted. Celeration lines indicate slope of change during each period, with the baseline celeration line extended into the intervention period for comparison. 


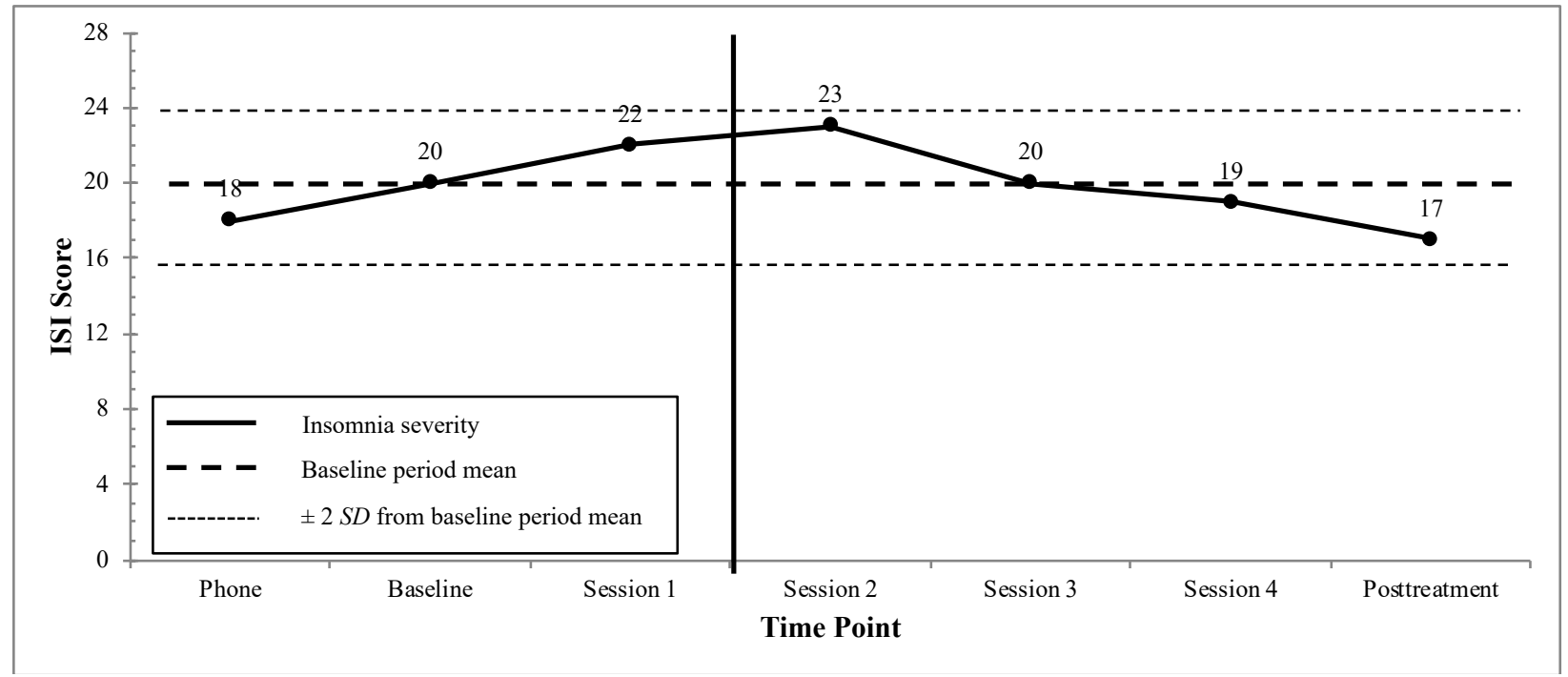

Figure 10a. Participant P6 Insomnia Severity Index scores with baseline period mean $\pm 2 S D$ plotted.

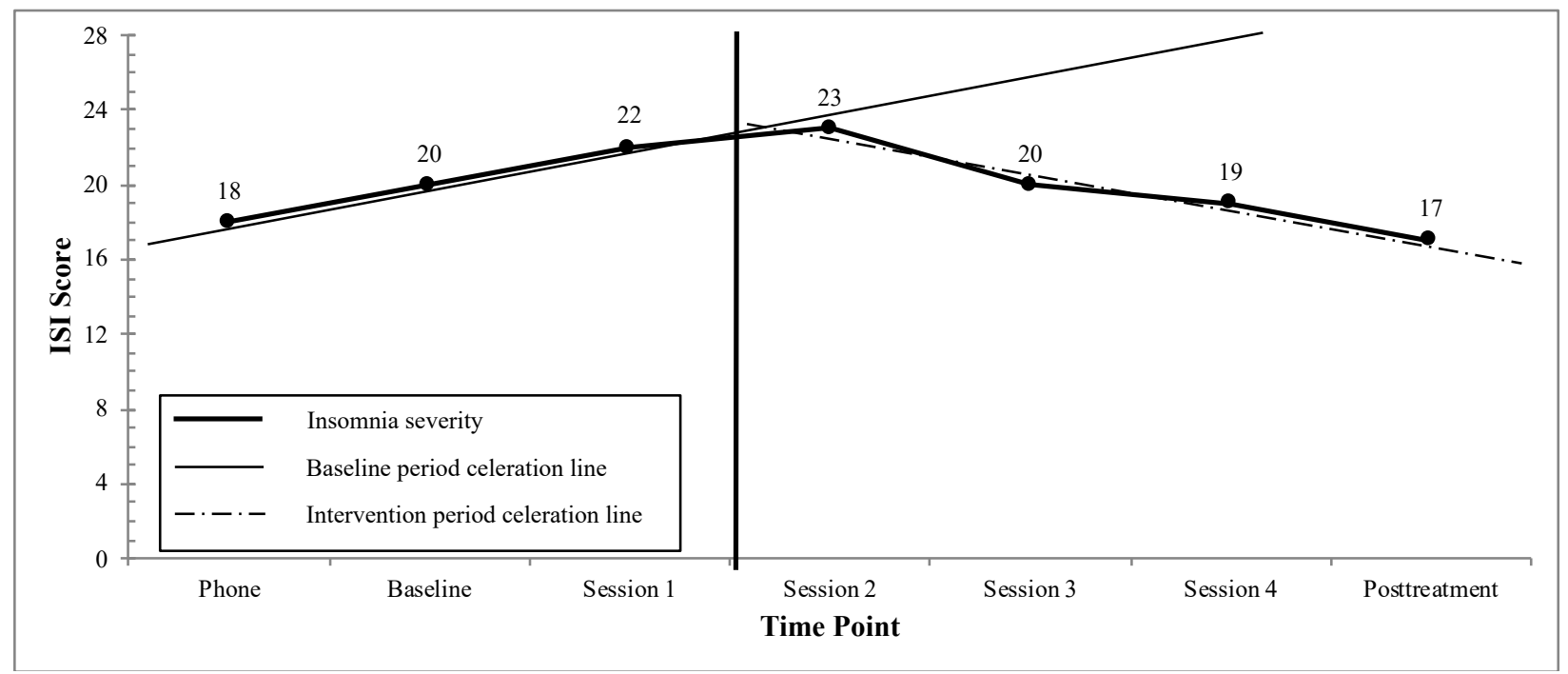

Figure 10b. Participant P6 Insomnia Severity Index scores with baseline and intervention period celeration lines plotted. Celeration lines indicate slope of change during each period, with the baseline celeration line extended into the intervention period for comparison. 


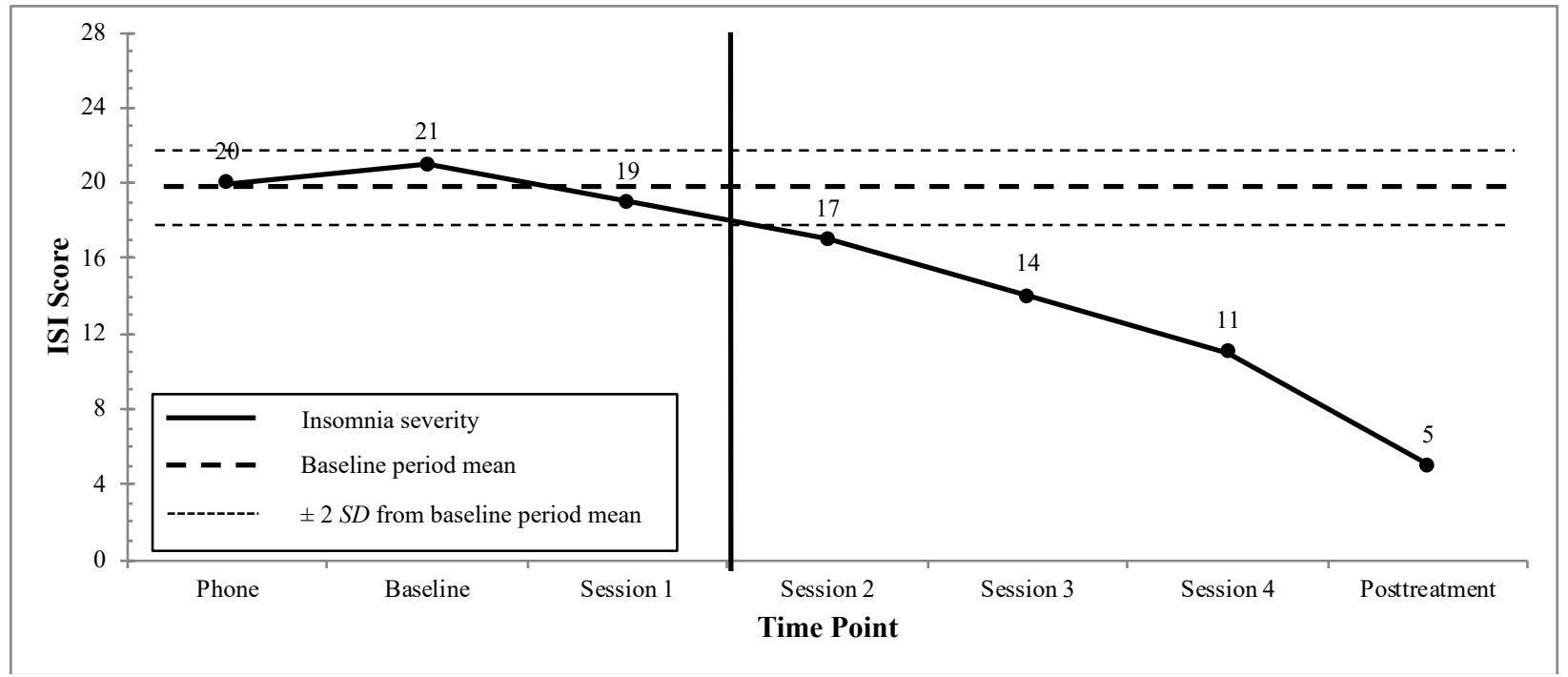

Figure 11a. Participant P7 Insomnia Severity Index scores with baseline period mean $\pm 2 S D$ plotted.

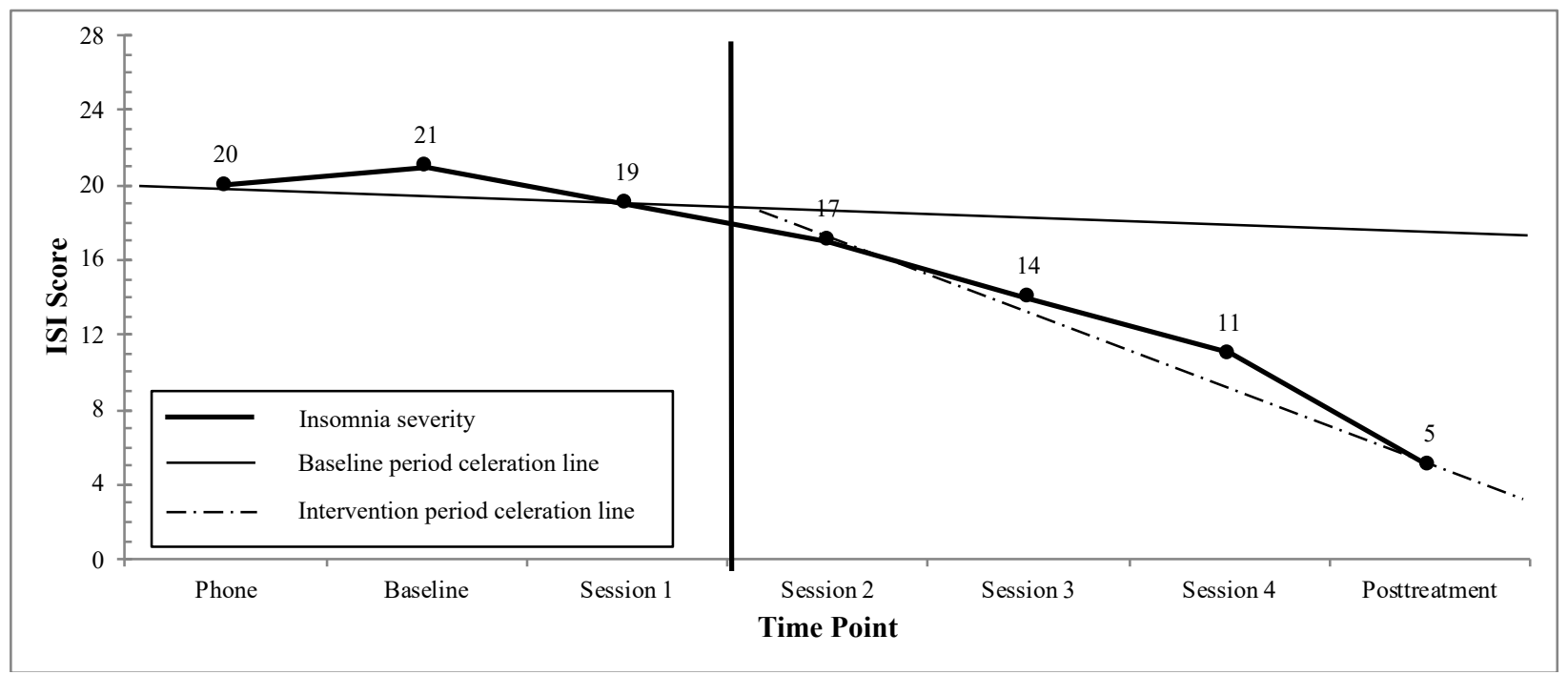

Figure 11b. Participant P7 Insomnia Severity Index scores with baseline and intervention period celeration lines plotted. Celeration lines indicate slope of change during each period, with the baseline celeration line extended into the intervention period for comparison. 


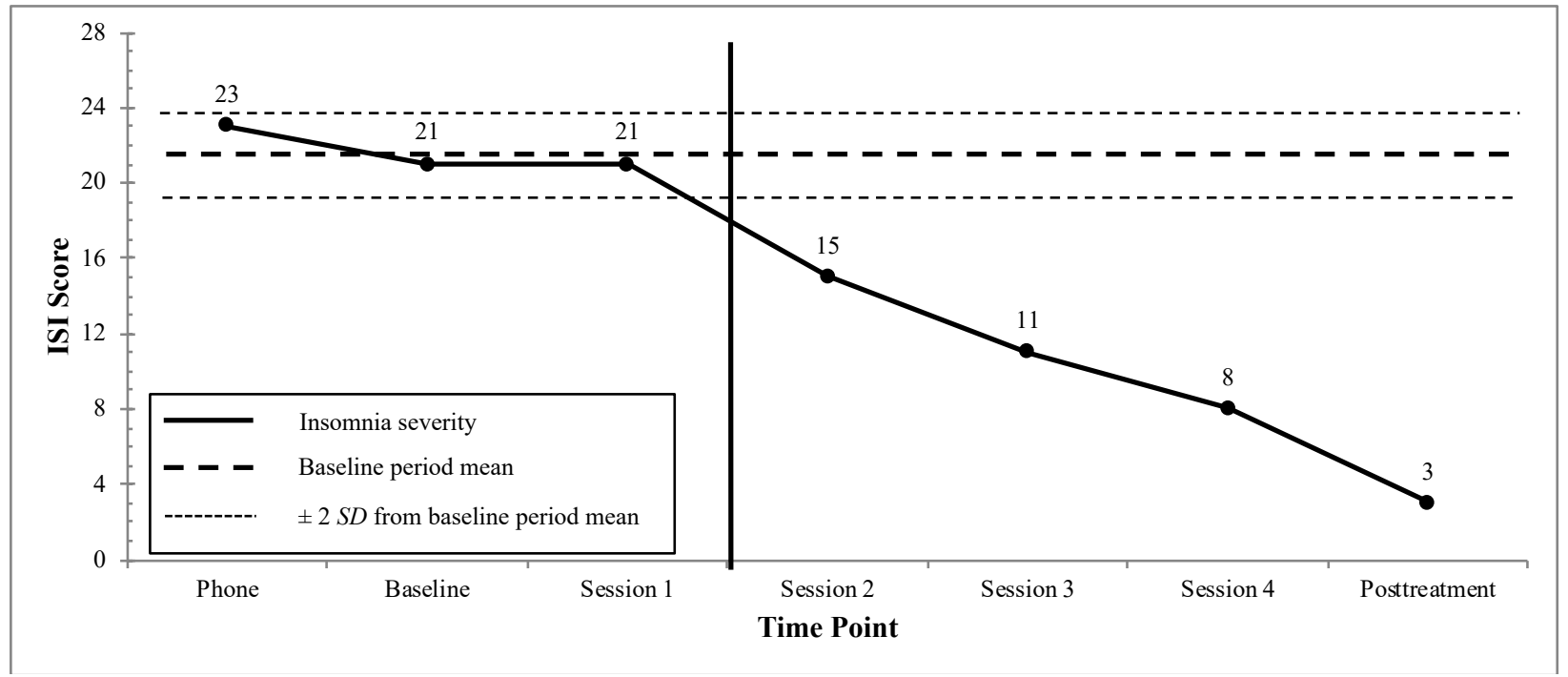

Figure12a. Participant P8 Insomnia Severity Index scores with baseline period mean $\pm 2 S D$ plotted.

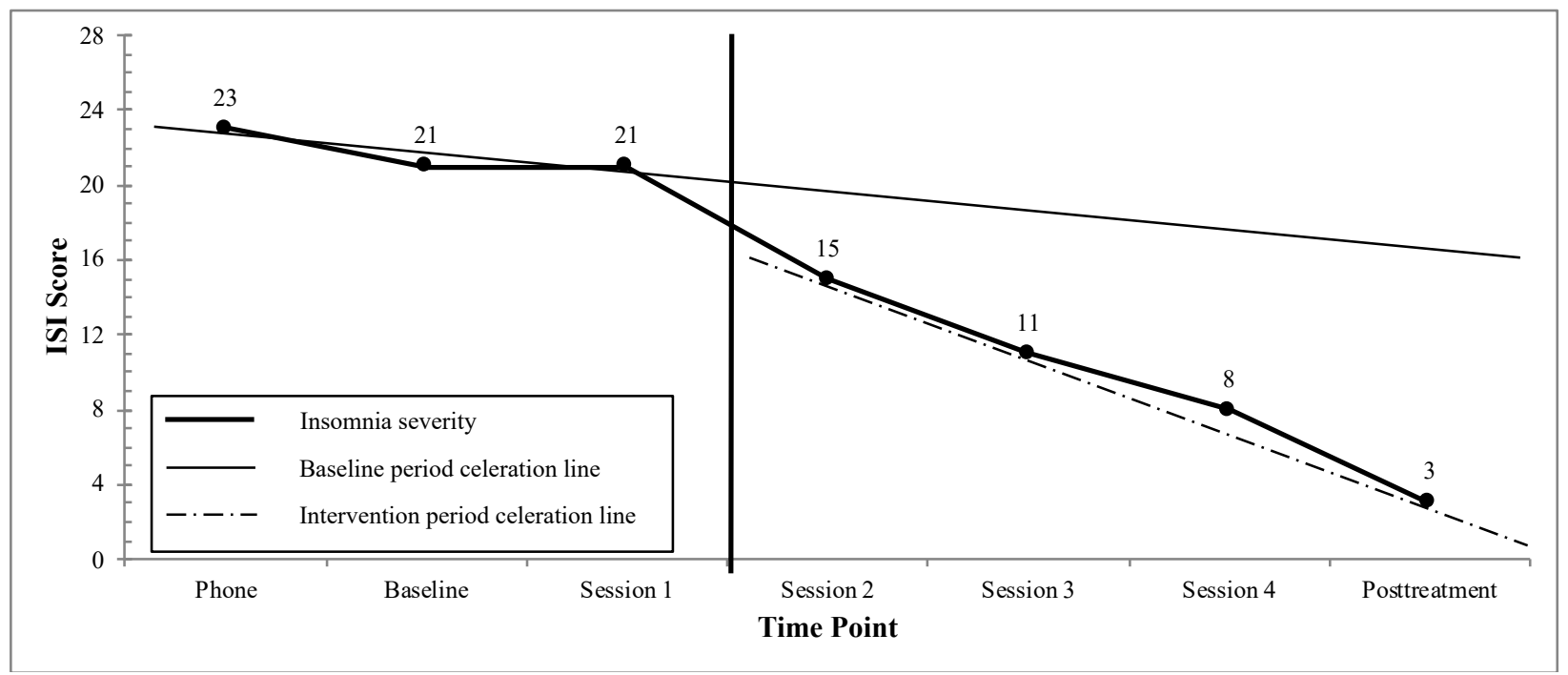

Figure 12b. Participant P8 Insomnia Severity Index scores with baseline and intervention period celeration lines plotted. Celeration lines indicate slope of change during each period, with the baseline celeration line extended into the intervention period for comparison. 


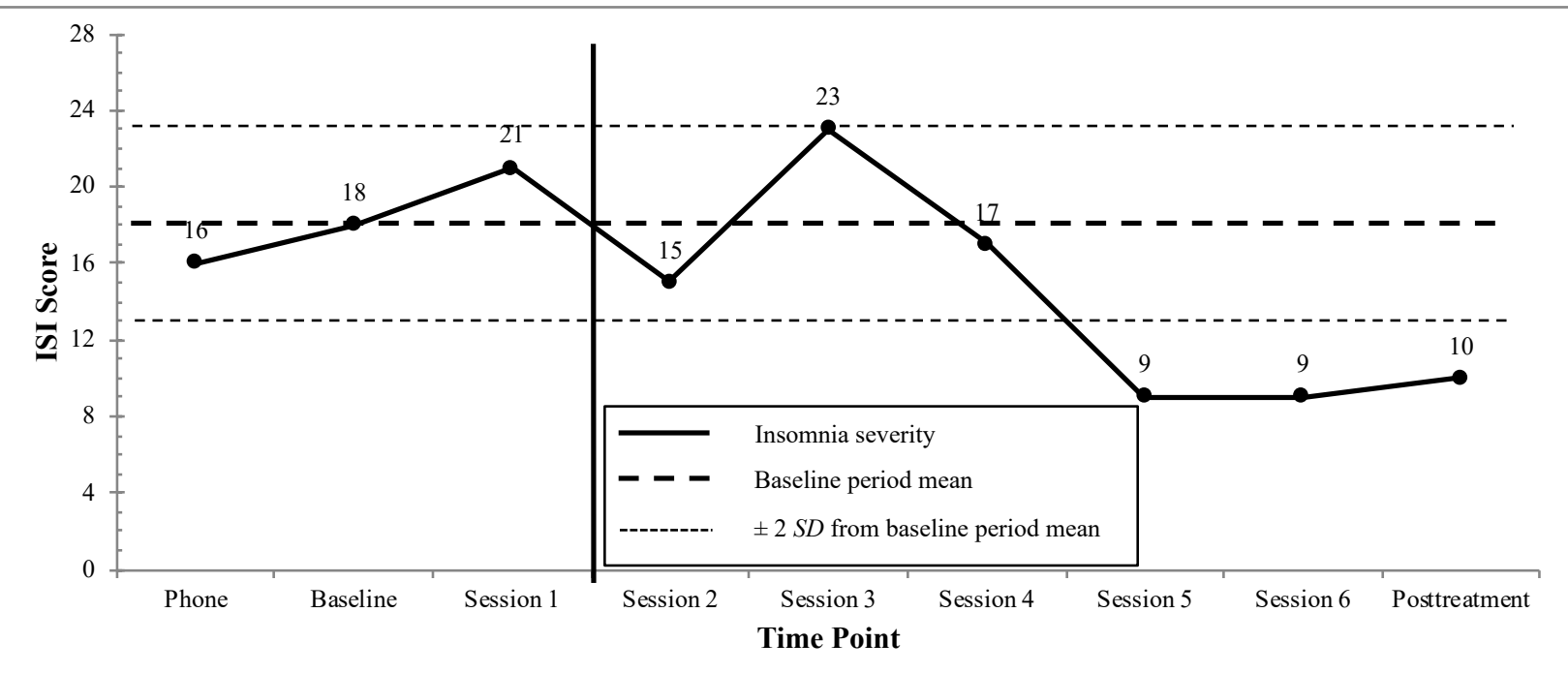

Figure 13a. Participant P9 Insomnia Severity Index scores with baseline period mean $\pm 2 S D$ plotted.

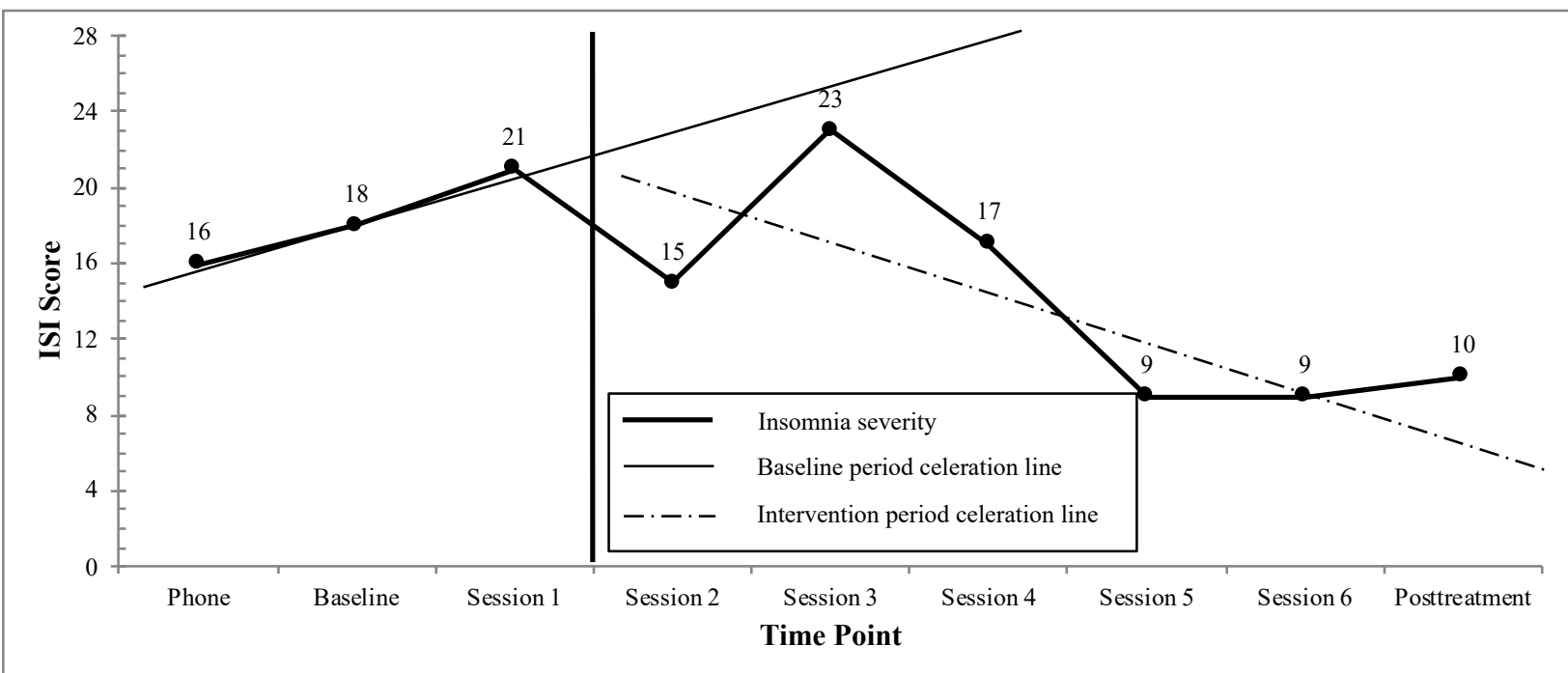

Figure 13b. Participant P9 Insomnia Severity Index scores with baseline and intervention period celeration lines plotted. Celeration lines indicate slope of change during each period, with the baseline celeration line extended into the intervention period for comparison. 


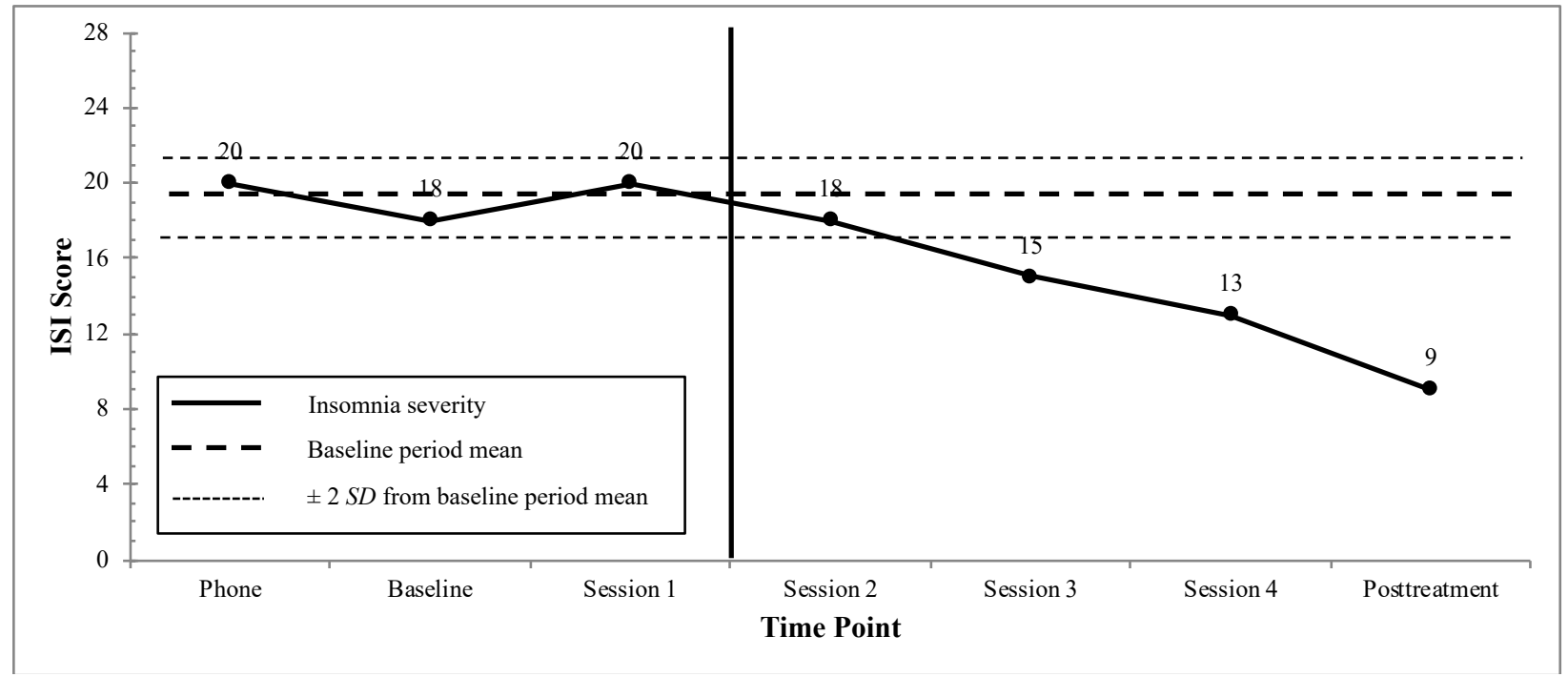

Figure 14a. Participant P10 Insomnia Severity Index scores with baseline period mean $\pm 2 S D$ plotted.

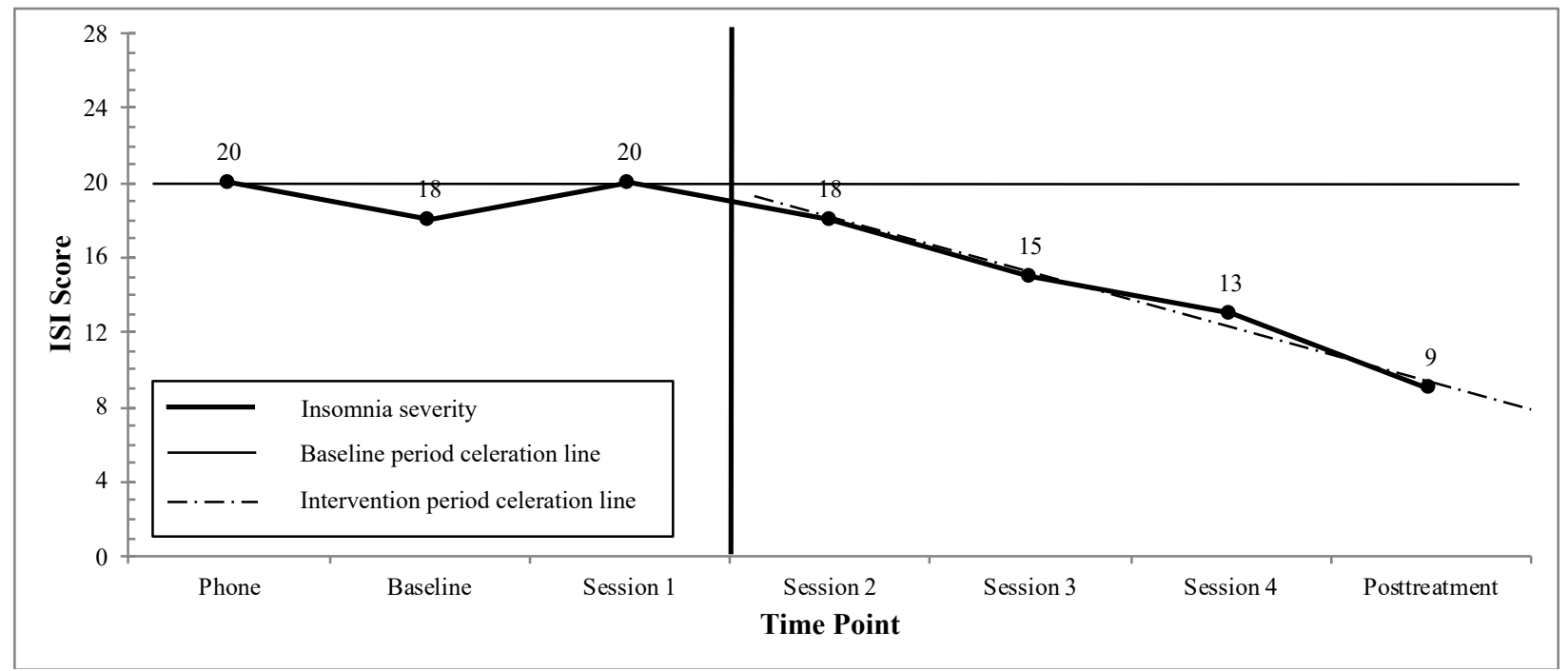

Figure 14b. Participant P10 Insomnia Severity Index scores with baseline and intervention period celeration lines plotted. Celeration lines indicate slope of change during each period, with the baseline celeration line extended into the intervention period for comparison. 
ISI, was supported using multiple analytic approaches, including visual inspection of magnitude and slope of change, comparison with markers of clinically significant change, and statistical significance testing.

Hypothesis 2: Sleep efficiency. Two-week mean sleep efficiency increased from 65\% $(S D=16 \%)$ at baseline to $89 \%(S D=6 \%)$ at posttreatment, within the target sleep efficiency range of $85 \%$ to $90 \%$. Cohen's $d$, based on the $S D$ of baseline sleep efficiency was $d=-1.50$, indicating a large effect of treatment. The number of data points analyzed using visual inspection ranged from five to seven per participant. All participants had at least two data points to serve as baseline comparison prior to receiving CBT-I. With regard to magnitude of change, visual inspection of sleep efficiency values for each participant relative to their respective baseline period $M$ and $\pm 2 S D$ revealed that for all but one participant (P6), sleep efficiency for at least the final two time points was $>2 S D$ above the baseline period mean. For participant P6, sleep efficiency at the second-last time point was exactly $2 S D$ above the baseline period mean. With regard to latency of change, a sustained sleep efficiency $>2 S D$ above the baseline period mean was observed after just one session for seven participants, after two sessions for one participant, after four sessions for one participant, and not at all for one participant. Mean sleep efficiency for the sample increased $>2 S D$ from the baseline period mean after just one session (Figure 15).

With regard to trend, visual inspection of celeration lines representing slope of sleep efficiency over time revealed a steeper upward slope in the intervention period relative to the baseline period for only one participant (P4). A ceiling effect was noted here, whereby slopes in the intervention period flattened out as sleep efficiency increased and approached or surpassed the target of $85 \%$, which is near the maximum score of $100 \%$. Therefore, visual analysis of celeration lines was not an effective visual aid for determining reliable change in sleep 


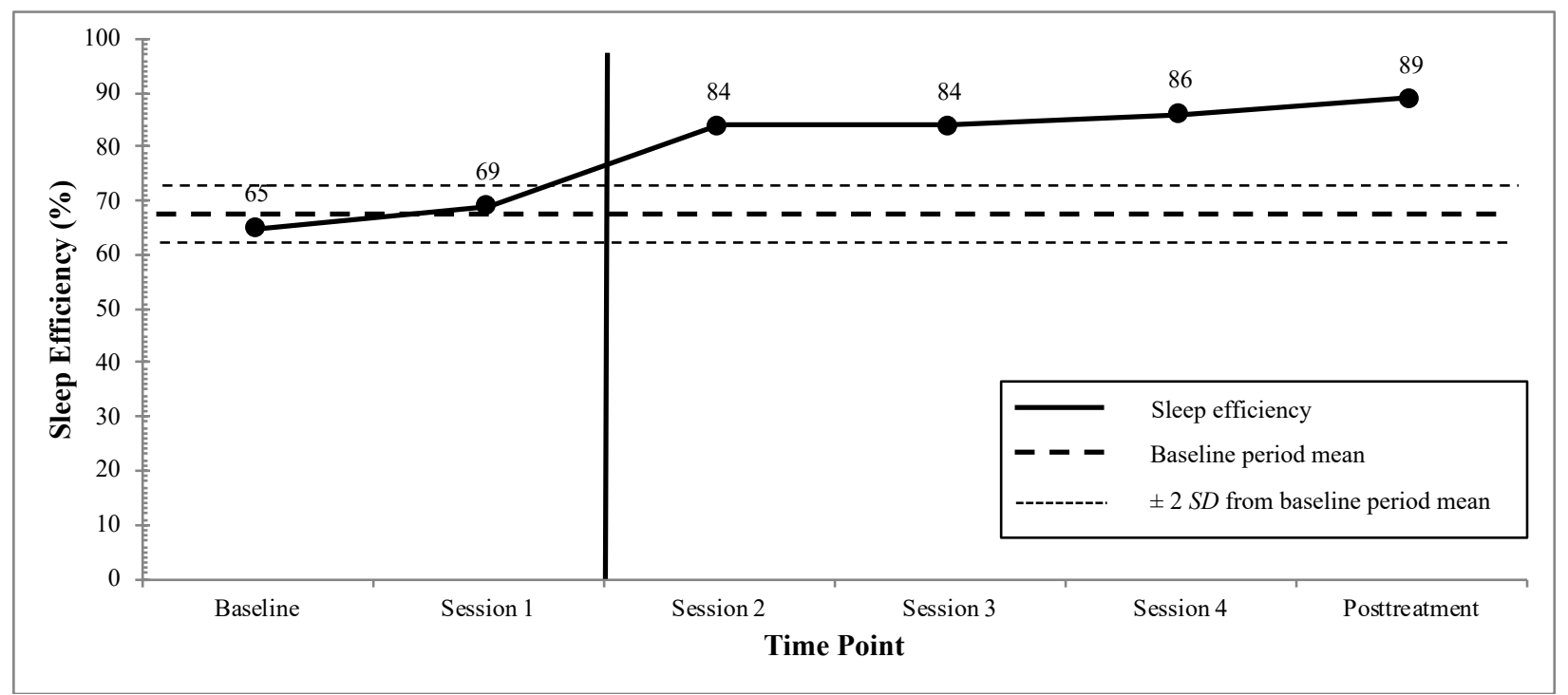

Figure 15a. Two-week mean sleep efficiency with baseline period mean $\pm 2 S D$ plotted, $n=10$.

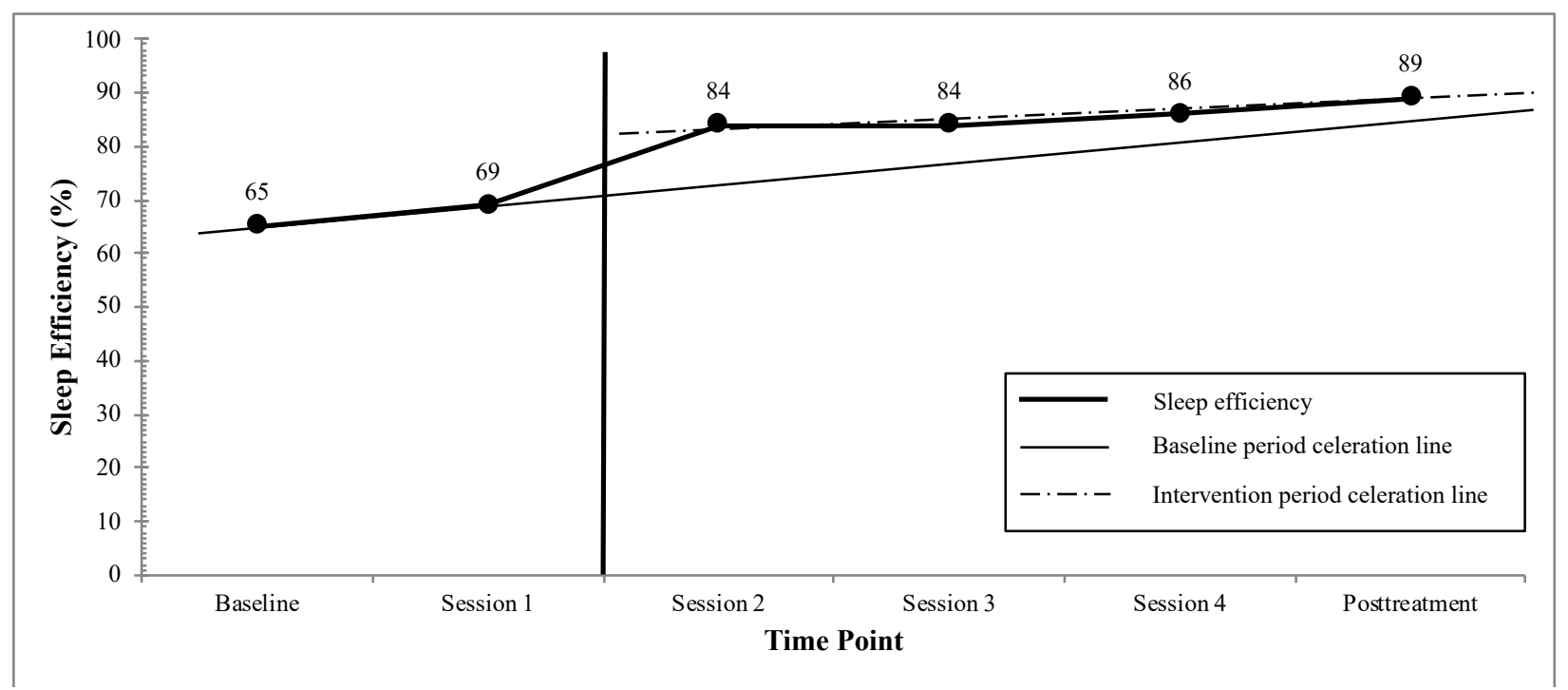

Figure 15b. Two-week mean sleep efficiency with baseline and intervention period celeration lines plotted. Celeration lines indicate slope of change during each period, with the baseline celeration line extended into the intervention period for comparison, $n=10$. 
efficiency. Reliable change in sleep efficiency based on magnitude of change was identified for nine of the ten participants, and for all participants when using a slightly less conservative criterion of $\geq 2 S D$ to identify reliable change. Figure 15 presents the sample mean sleep efficiency at each time point, accompanied by visual aids, and sleep efficiency change for each participant is presented in Figures 16-25.

With regard to clinically significant change, only two participants were within the target sleep efficiency range of $85 \%$ to $90 \%$ at posttreatment. Five participants surpassed the target range with sleep efficiency $>90 \%$, which, although no longer indicative of insomnia, suggests the presence of sleep deprivation and the need to continue increasing the amount of time spent in bed until sleep efficiency decreases to within the target range. For three participants, sleep efficiency was below the target range, but only one participant's sleep efficiency remained < $80 \%$, within the range indicative of insomnia.

Exploratory paired samples $t$-tests applying bias-corrected bootstrapping to account for non-normally distributed data demonstrated that sleep efficiency significantly increased from baseline $(M=.65, S D=.16)$ to posttreatment $(M=.89, S D=.06), t(9)=-4.42, p=.002$, biascorrected $95 \%$ CI $[-.38,-.16]$. The second hypothesis, that participants would experience an improvement in sleep efficiency, with a target value between $85 \%$ and $90 \%$, was partially supported using multiple analytic approaches, including visual inspection of magnitude of change, comparison with a target range, and statistical significance testing. Although sleep efficiency for three participants was below the target range at posttreatment, it was within or above the target range for the remaining participants, and the mean sleep efficiency for the group at posttreatment was within the target range. Furthermore, a statistically significant increase in sleep efficiency was observed. 


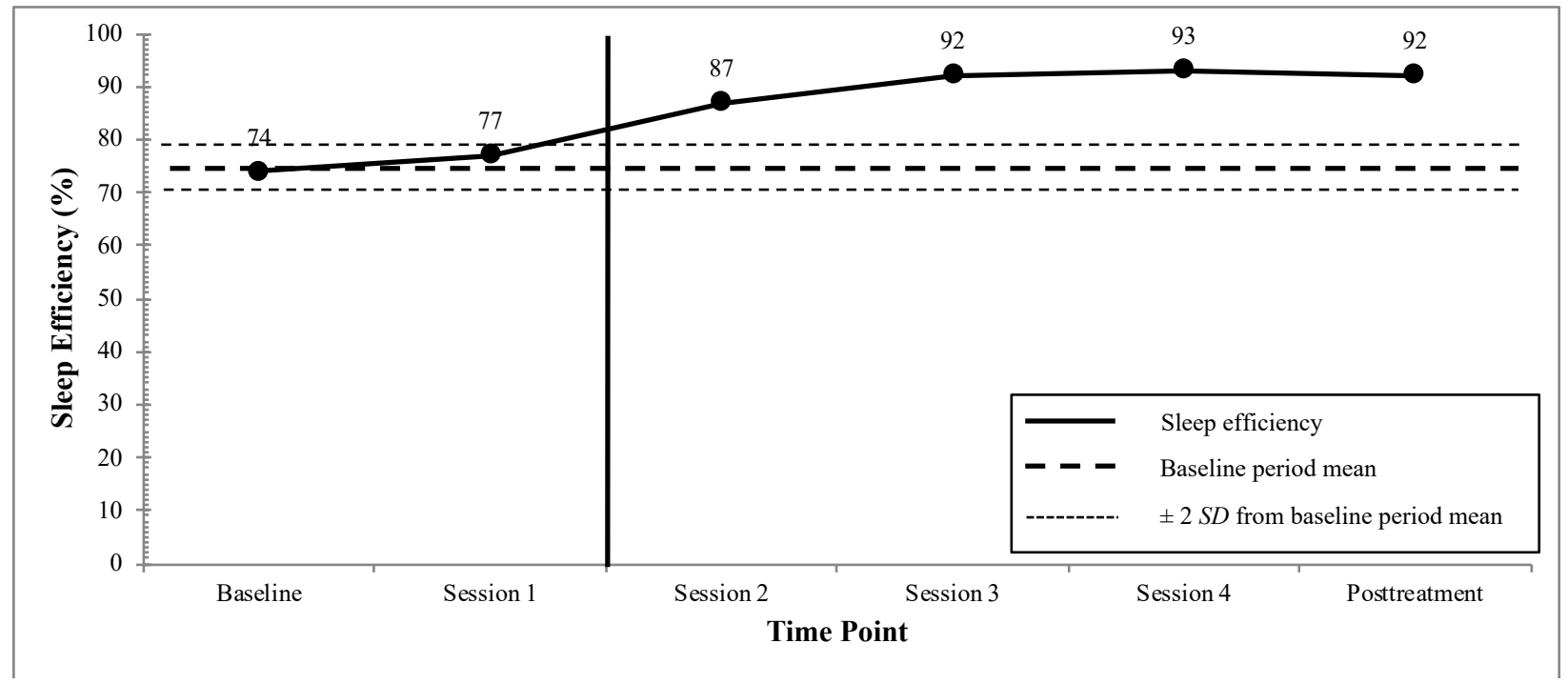

Figure 16a. Participant P1 two-week mean sleep efficiency with baseline period mean $\pm 2 S D$ plotted.

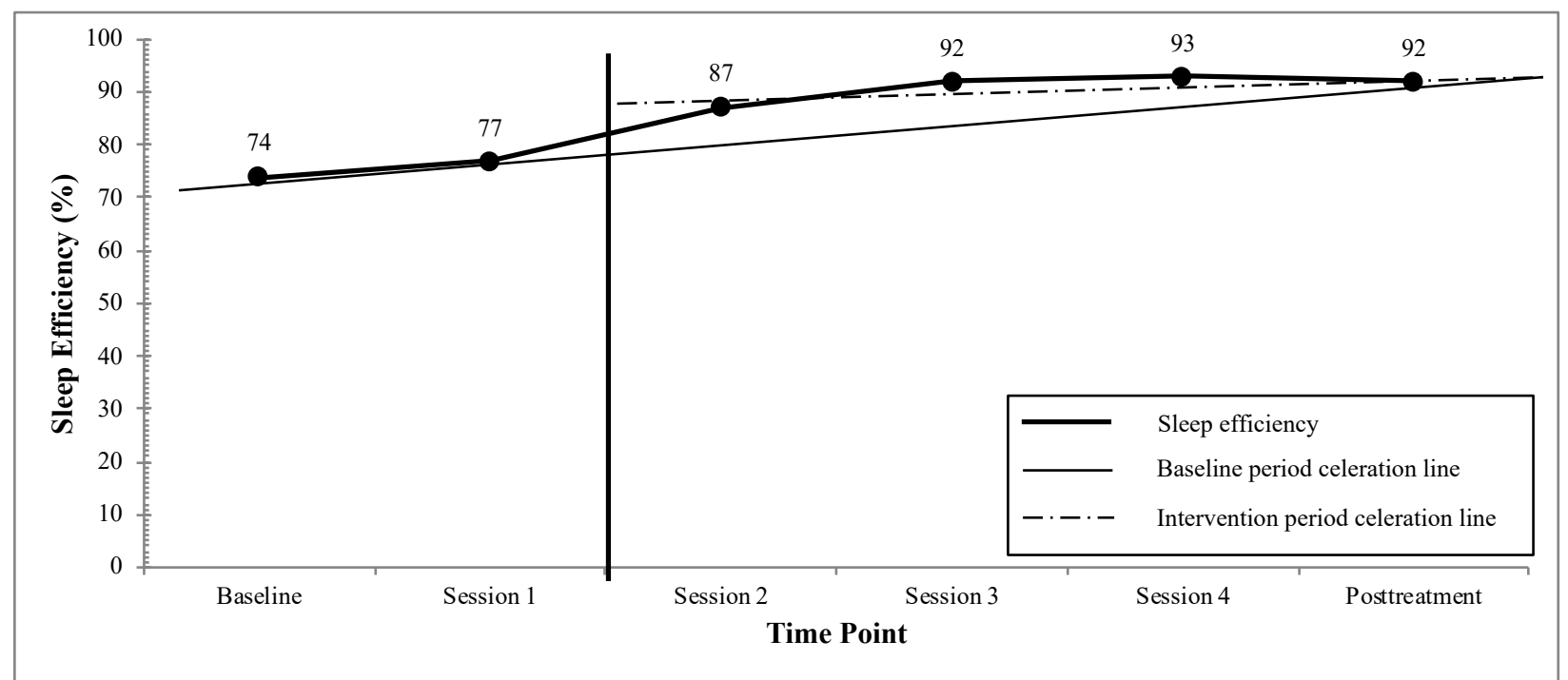

Figure 16b. Participant P1 two-week mean sleep efficiency with baseline and intervention period celeration lines plotted. Celeration lines indicate slope of change during each period, with the baseline celeration line extended into the intervention period for comparison. 


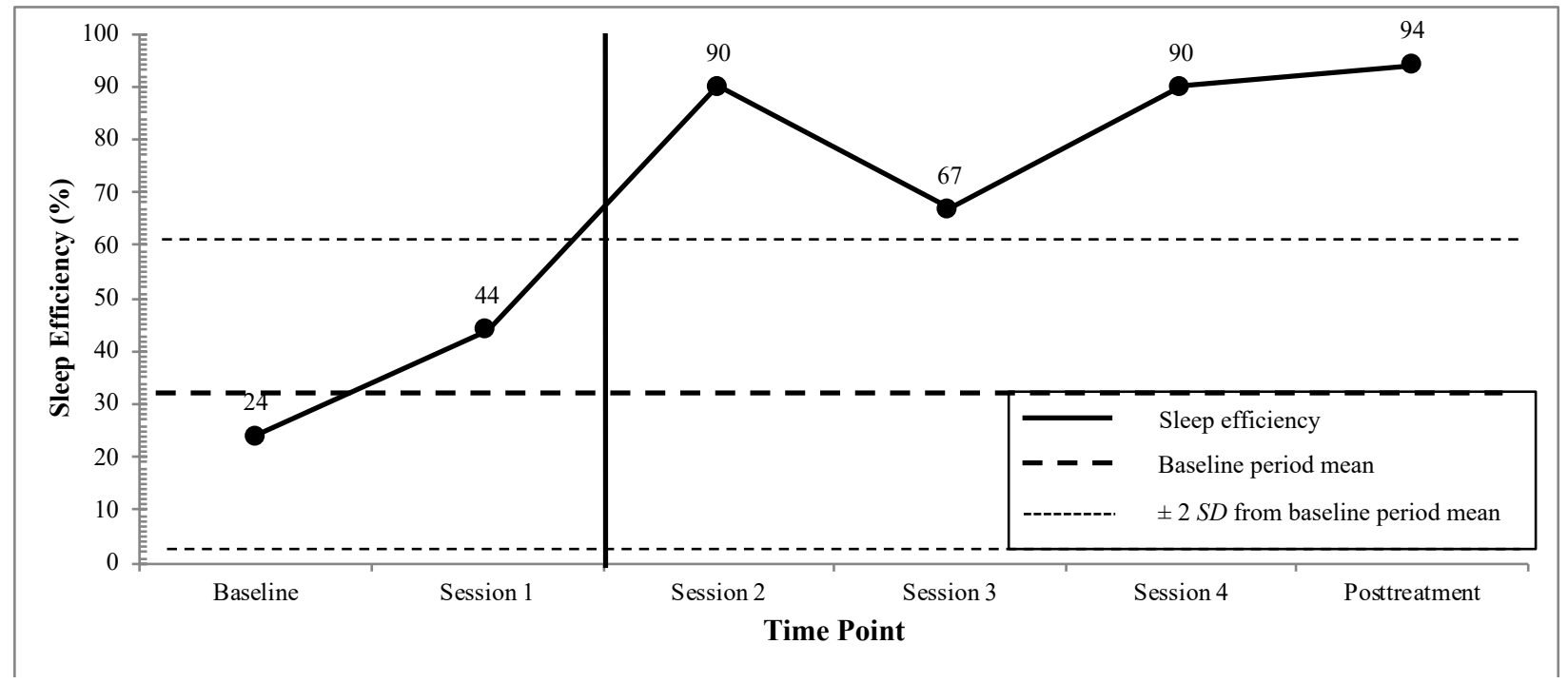

Figure 17a. Participant P2 two-week mean sleep efficiency with baseline period mean $\pm 2 S D$ plotted.

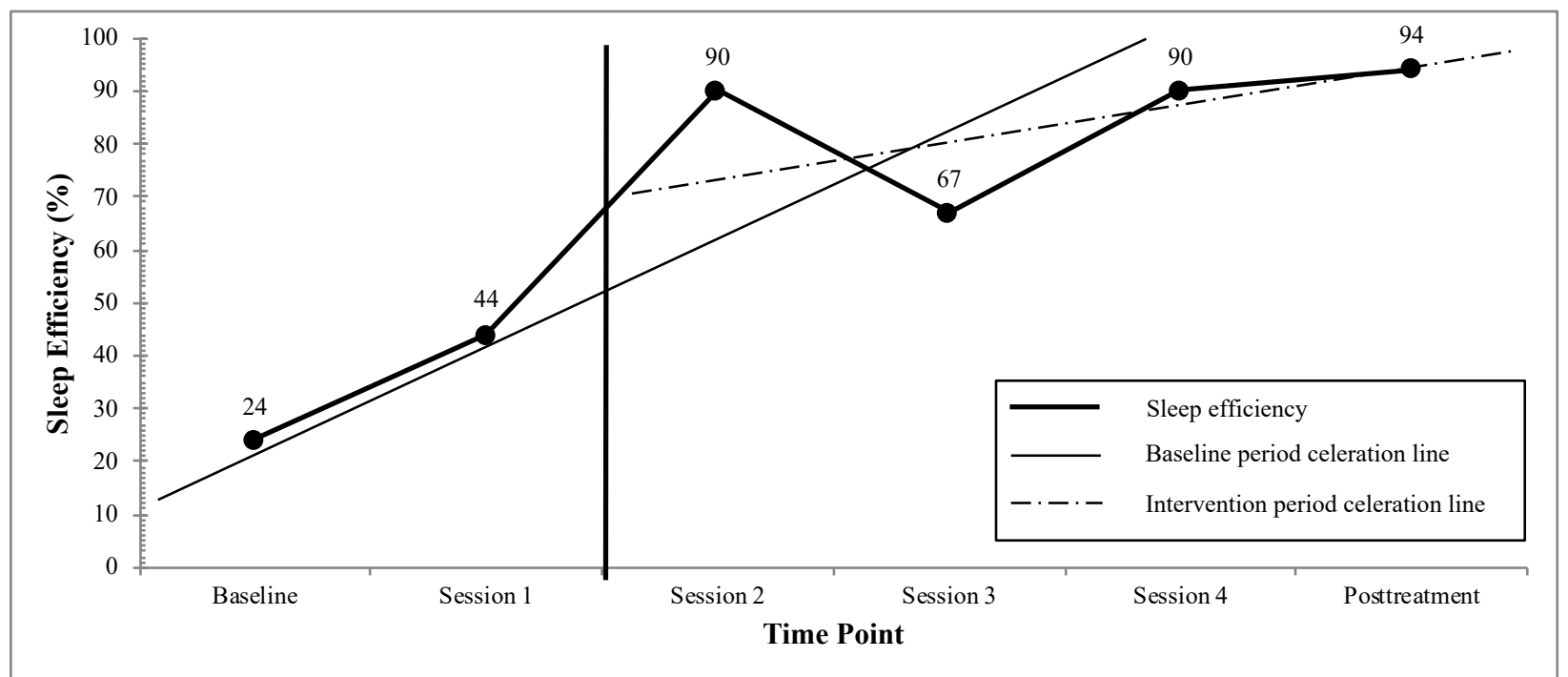

Figure 17b. Participant P2 two-week mean sleep efficiency with baseline and intervention period celeration lines plotted. Celeration lines indicate slope of change during each period, with the baseline celeration line extended into the intervention period for comparison. 


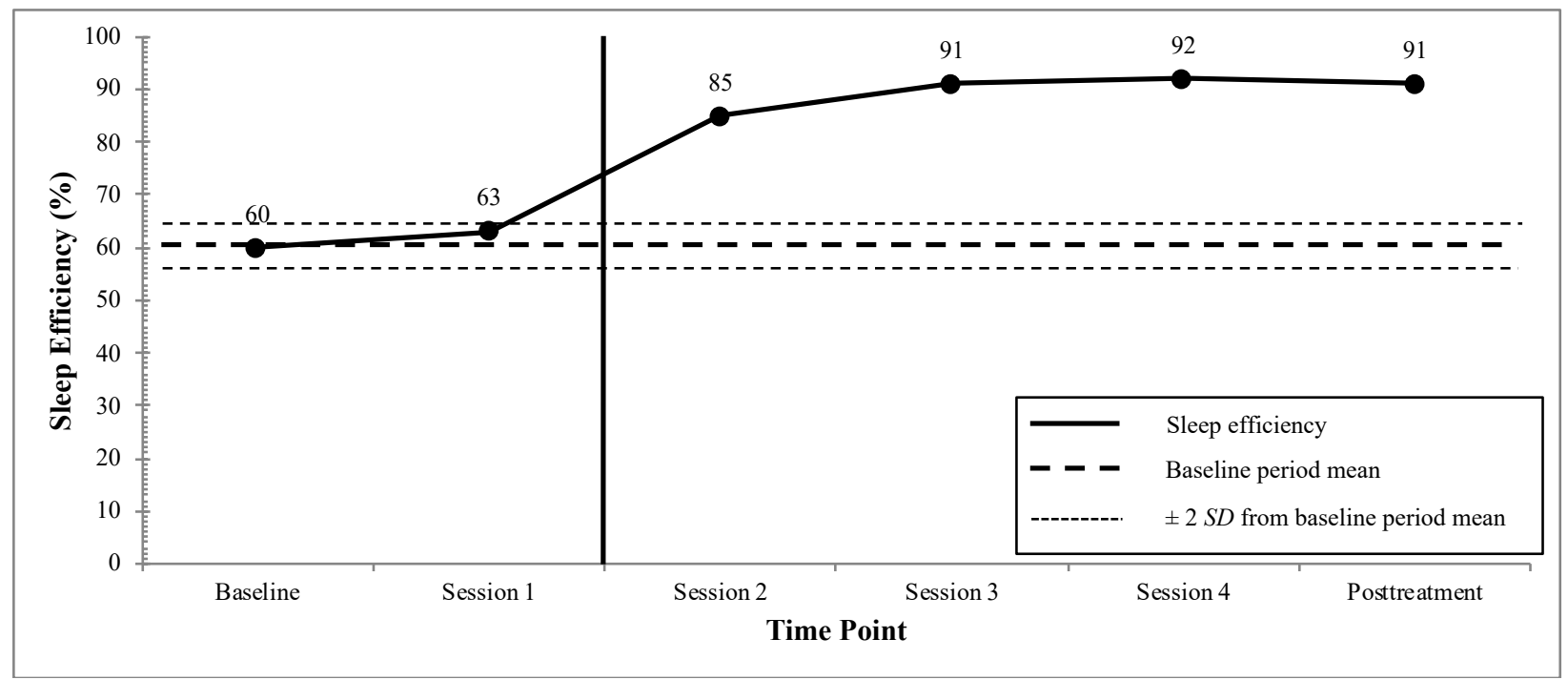

Figure 18a. Participant P3 two-week mean sleep efficiency with baseline period mean $\pm 2 S D$ plotted.

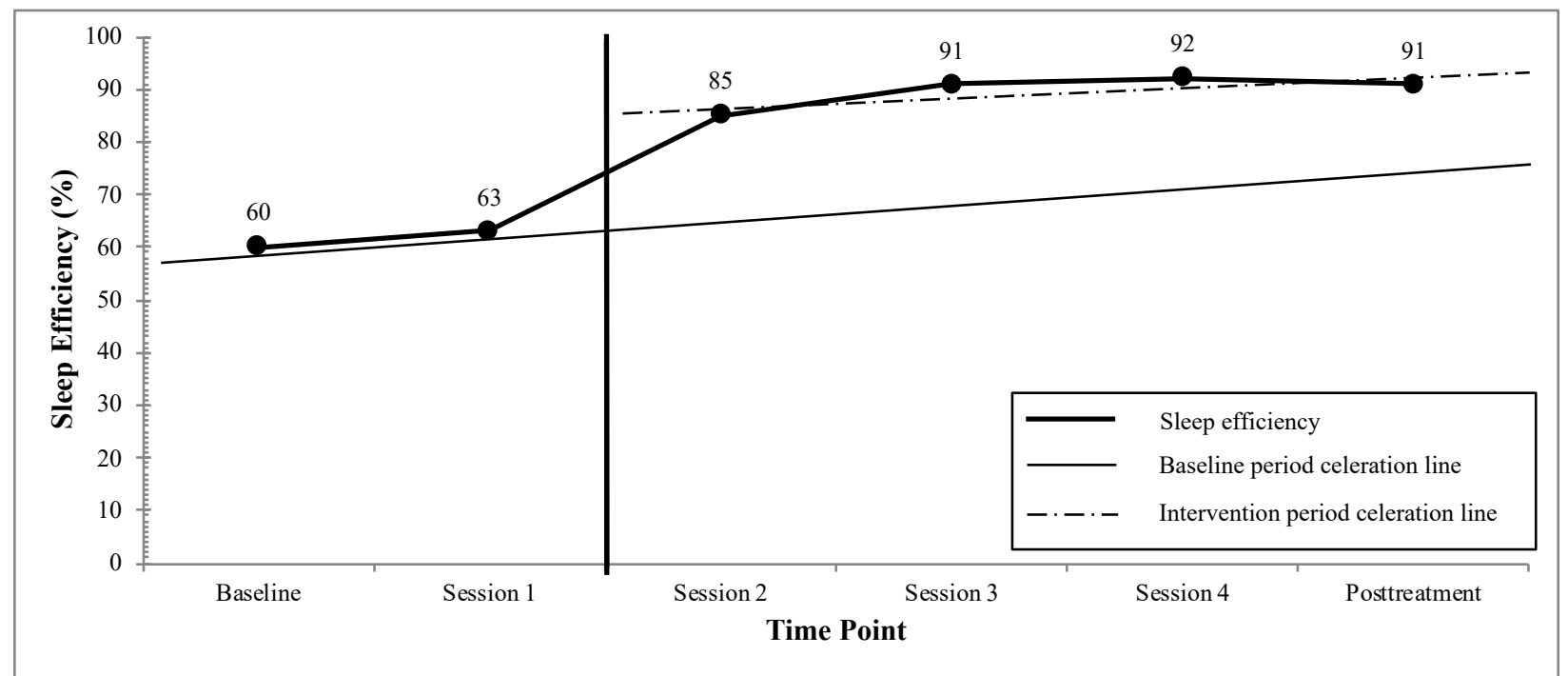

Figure 18b. Participant P3 two-week mean sleep efficiency with baseline and intervention period celeration lines plotted. Celeration lines indicate slope of change during each period, with the baseline celeration line extended into the intervention period for comparison. 


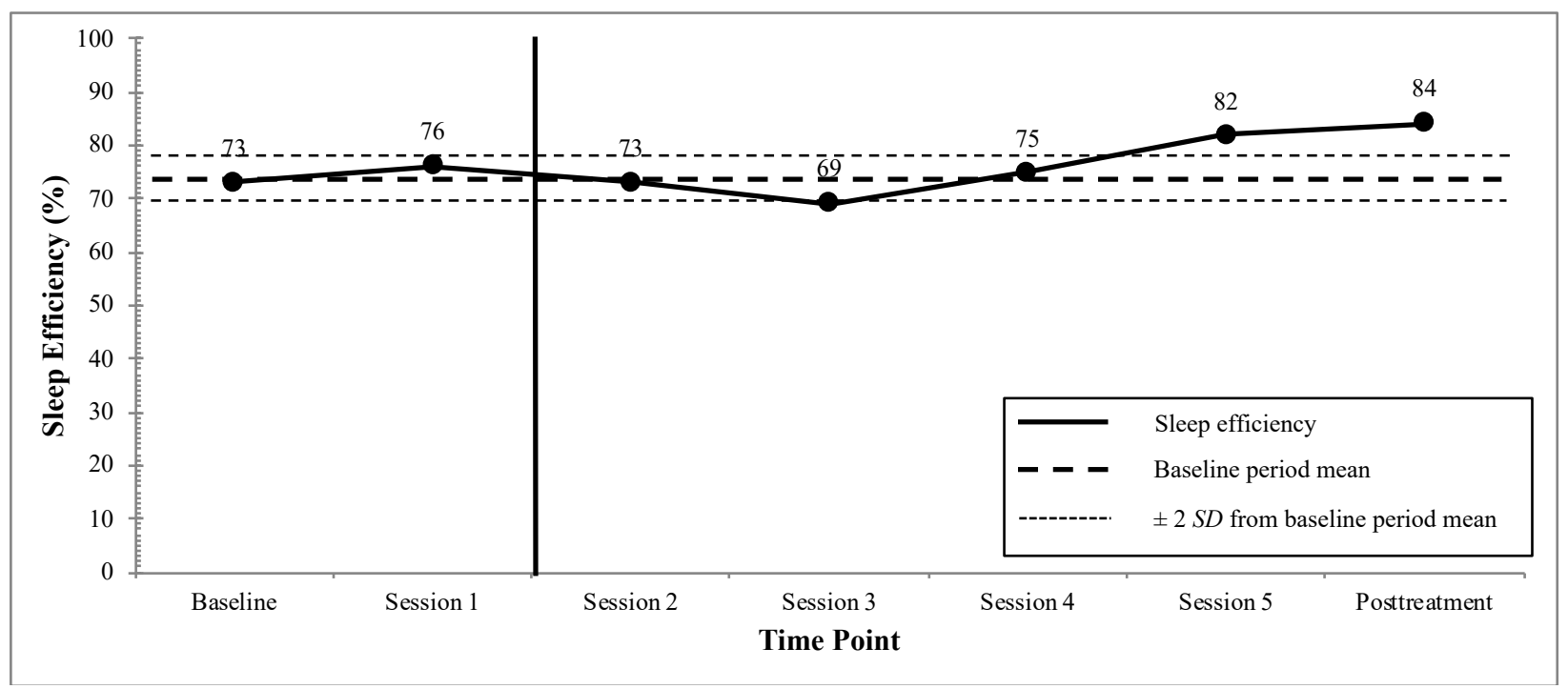

Figure 19a. Participant P4 two-week mean sleep efficiency with baseline period mean $\pm 2 S D$ plotted.

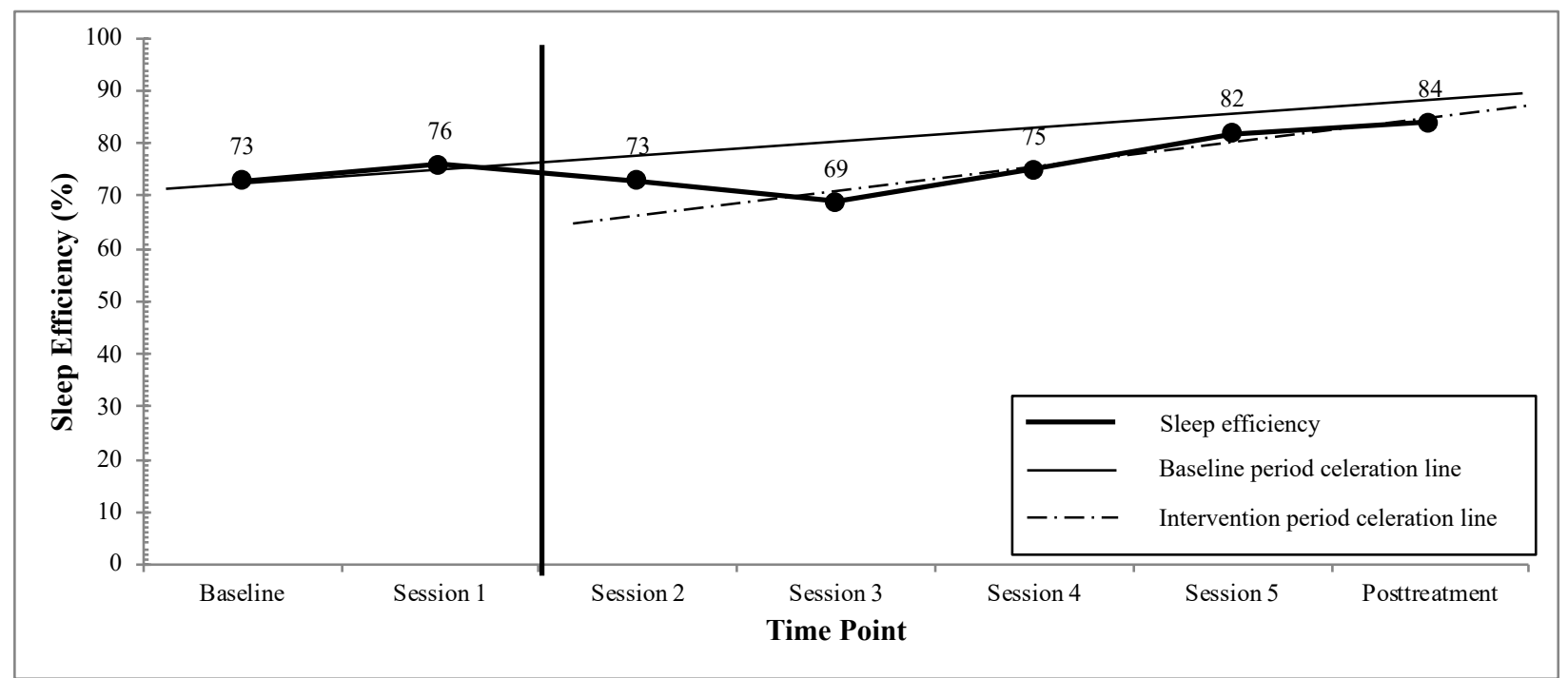

Figure 19b. Participant P4 two-week mean sleep efficiency with baseline and intervention period celeration lines plotted. Celeration lines indicate slope of change during each period, with the baseline celeration line extended into the intervention period for comparison. 


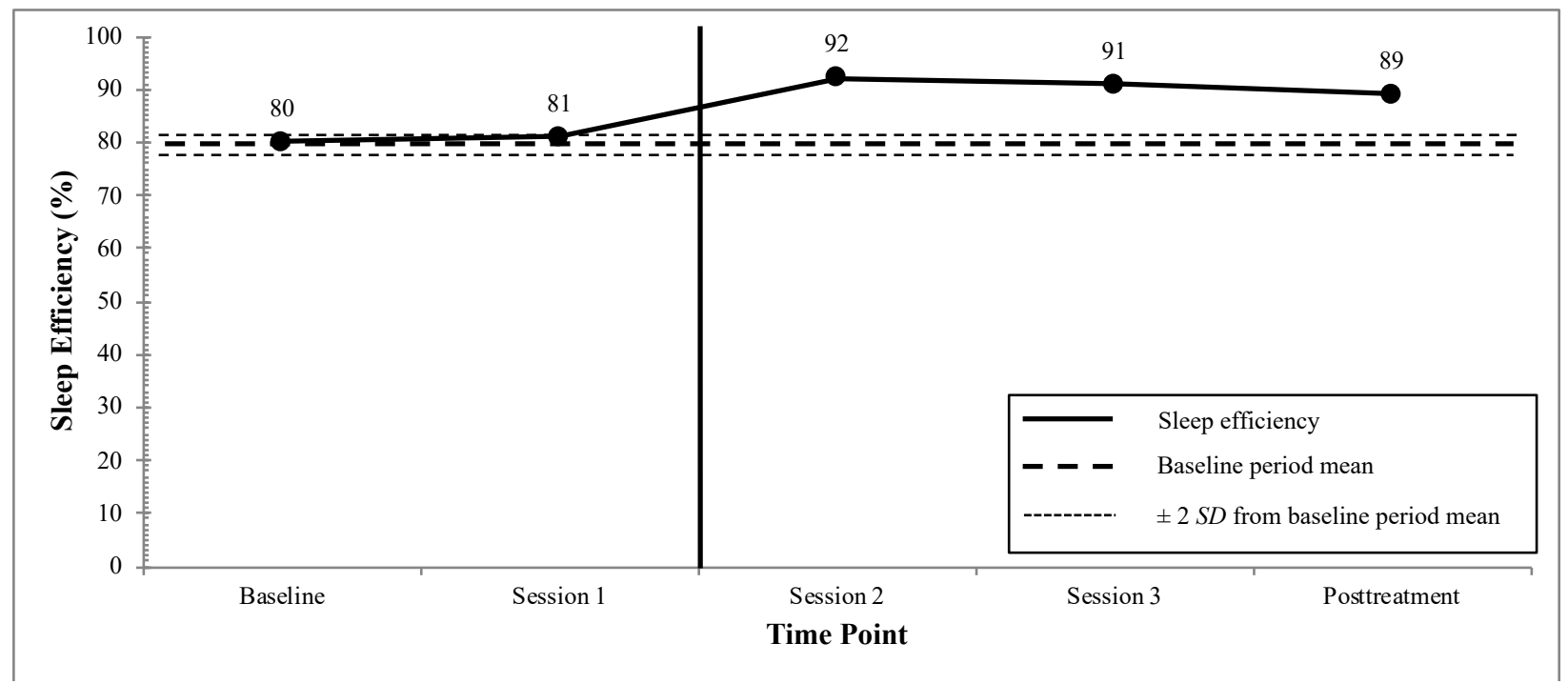

Figure 20a. Participant P5 two-week mean sleep efficiency with baseline period mean $\pm 2 S D$ plotted.

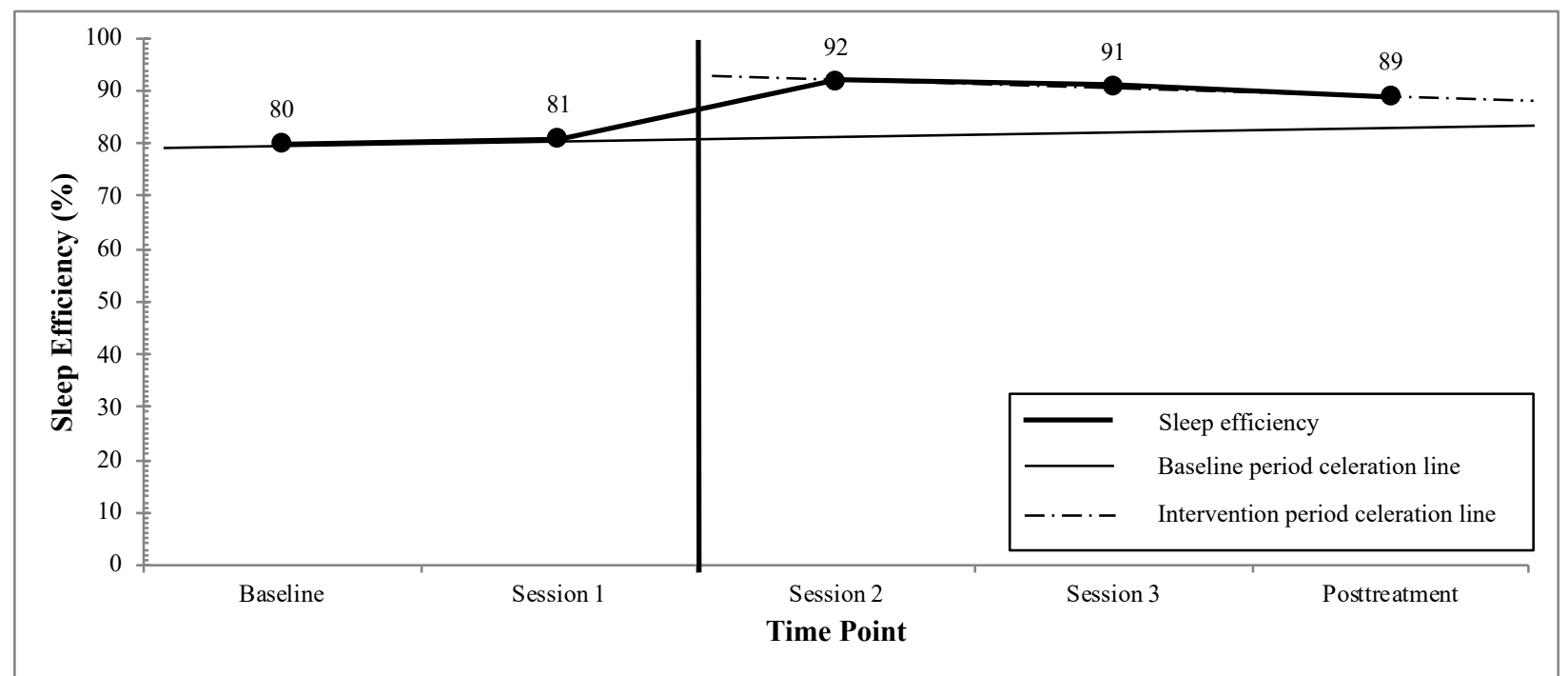

Figure 20b. Participant P5 two-week mean sleep efficiency with baseline and intervention period celeration lines plotted. Celeration lines indicate slope of change during each period, with the baseline celeration line extended into the intervention period for comparison. 


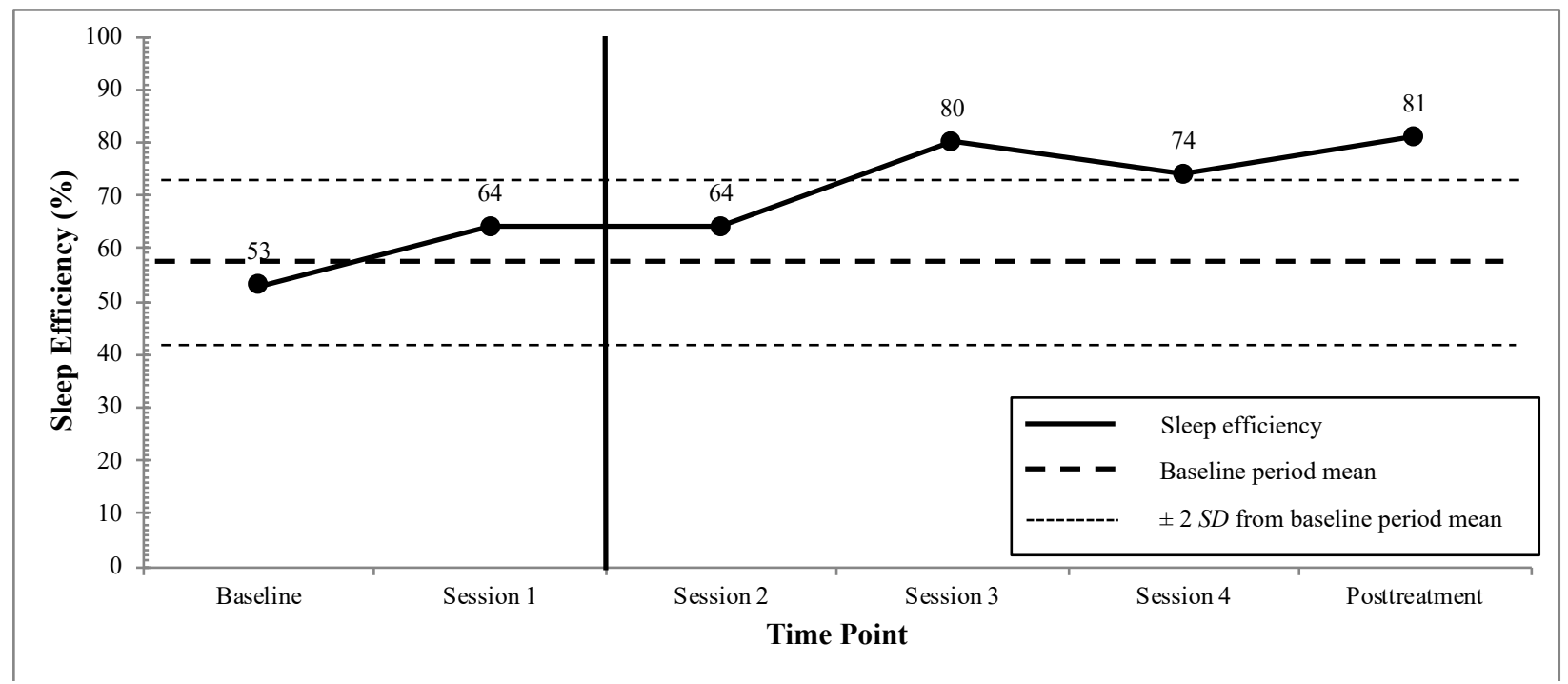

Figure 21a. Participant P6 two-week mean sleep efficiency with baseline period mean $\pm 2 S D$ plotted.

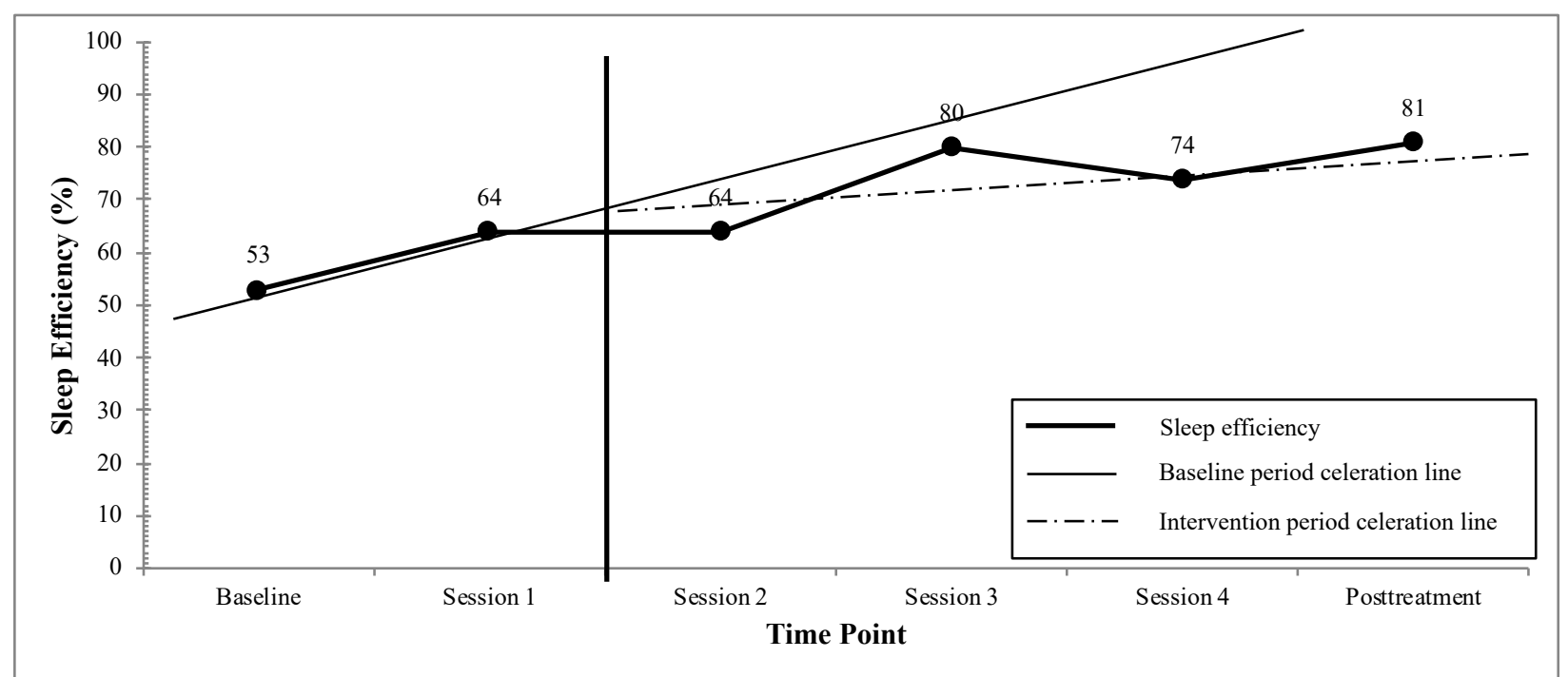

Figure 21b. Participant P6 two-week mean sleep efficiency with baseline and intervention period celeration lines plotted. Celeration lines indicate slope of change during each period, with the baseline celeration line extended into the intervention period for comparison. 


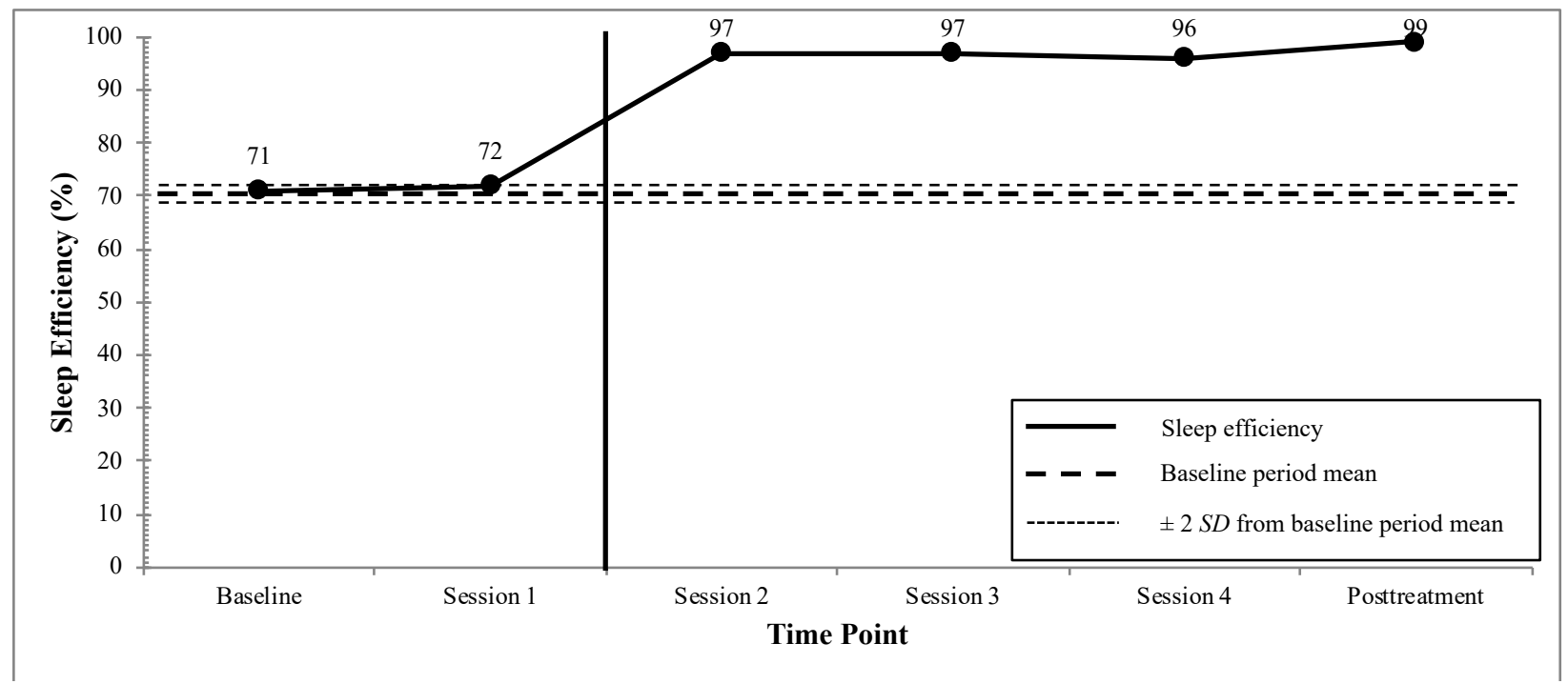

Figure 22a. Participant P7 two-week mean sleep efficiency with baseline period mean $\pm 2 S D$ plotted.

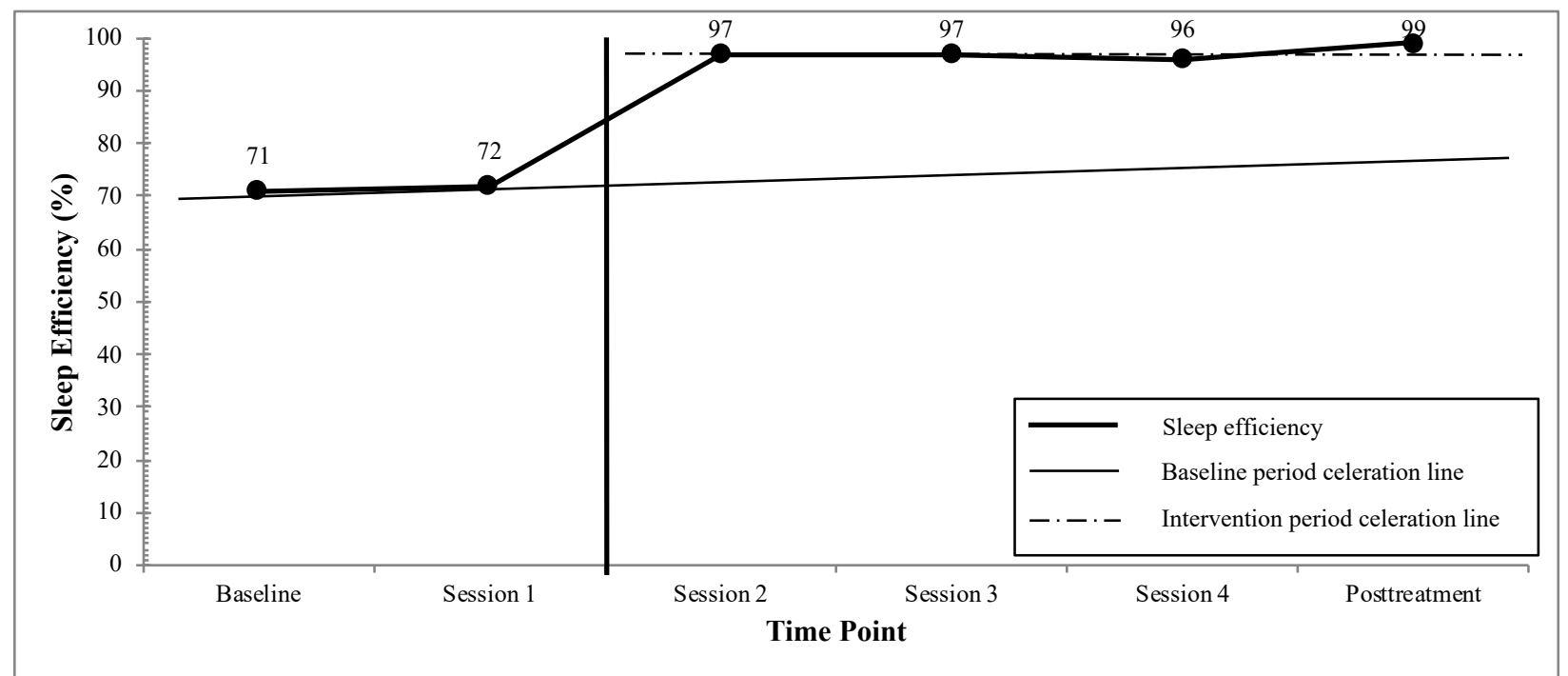

Figure 22b. Participant P7 two-week mean sleep efficiency with baseline and intervention period celeration lines plotted. Celeration lines indicate slope of change during each period, with the baseline celeration line extended into the intervention period for comparison. 


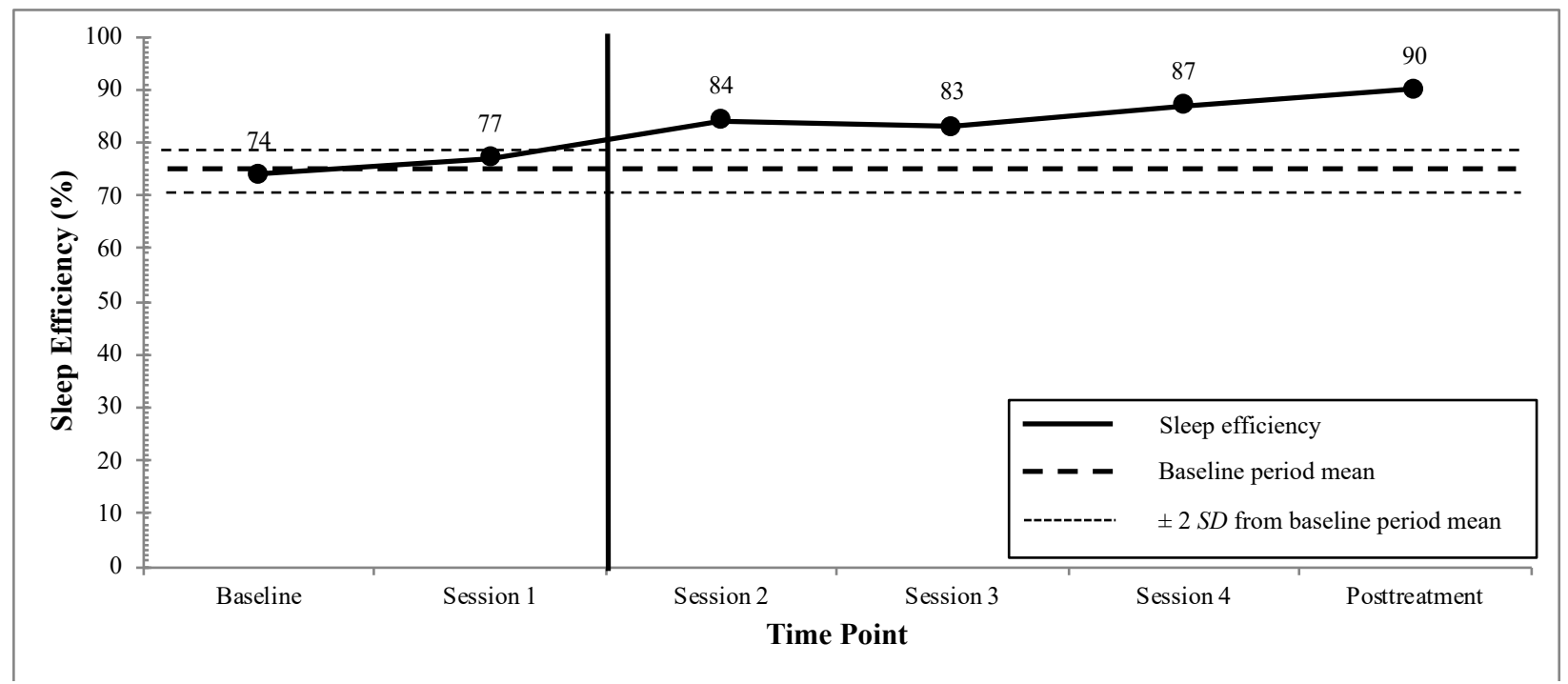

Figure 23a. Participant P8 two-week mean sleep efficiency with baseline period mean $\pm 2 S D$ plotted.

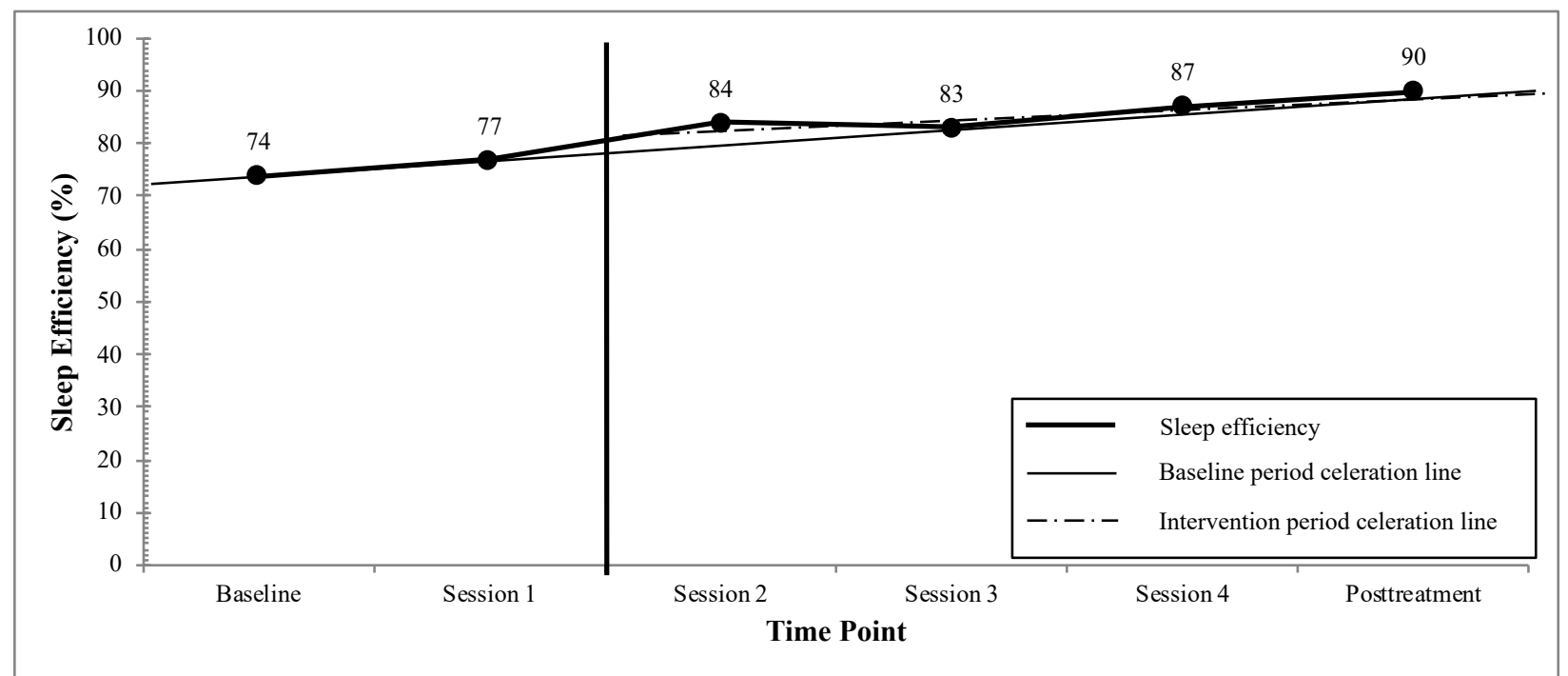

Figure 23b. Participant P8 two-week mean sleep efficiency with baseline and intervention period celeration lines plotted. Celeration lines indicate slope of change during each period, with the baseline celeration line extended into the intervention period for comparison. 


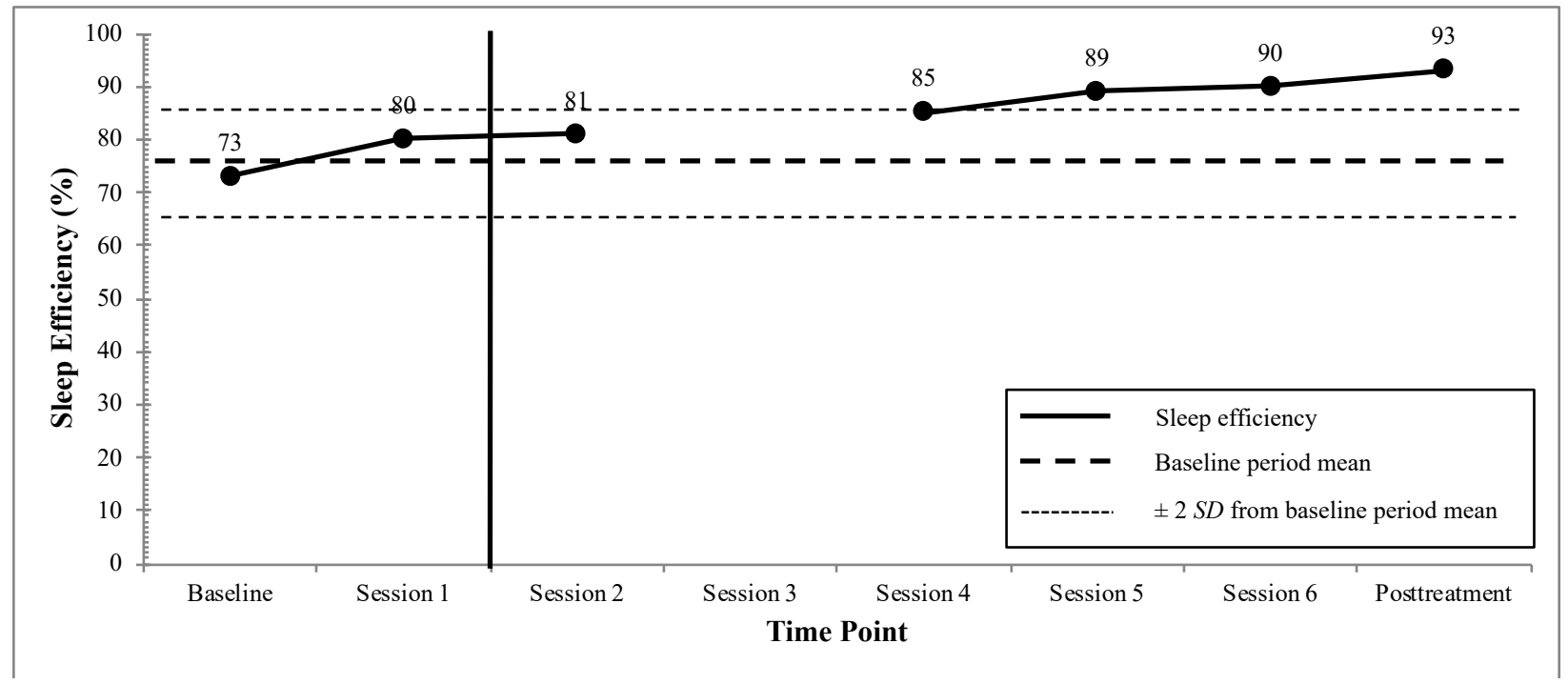

Figure 24a. Participant P9 two-week mean sleep efficiency with baseline period mean $\pm 2 S D$ plotted.

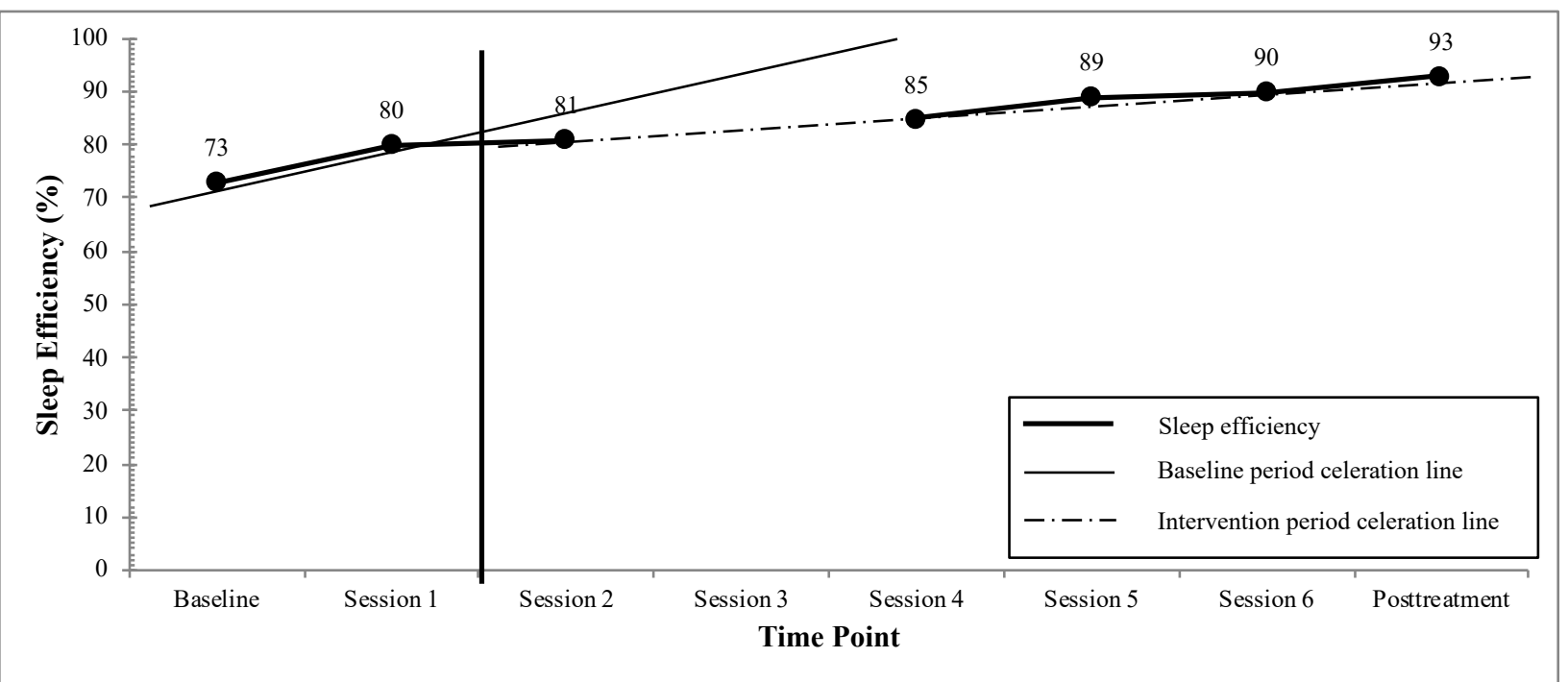

Figure 24b. Participant P9 two-week mean sleep efficiency with baseline and intervention period celeration lines plotted. Celeration lines indicate slope of change during each period, with the baseline celeration line extended into the intervention period for comparison. 


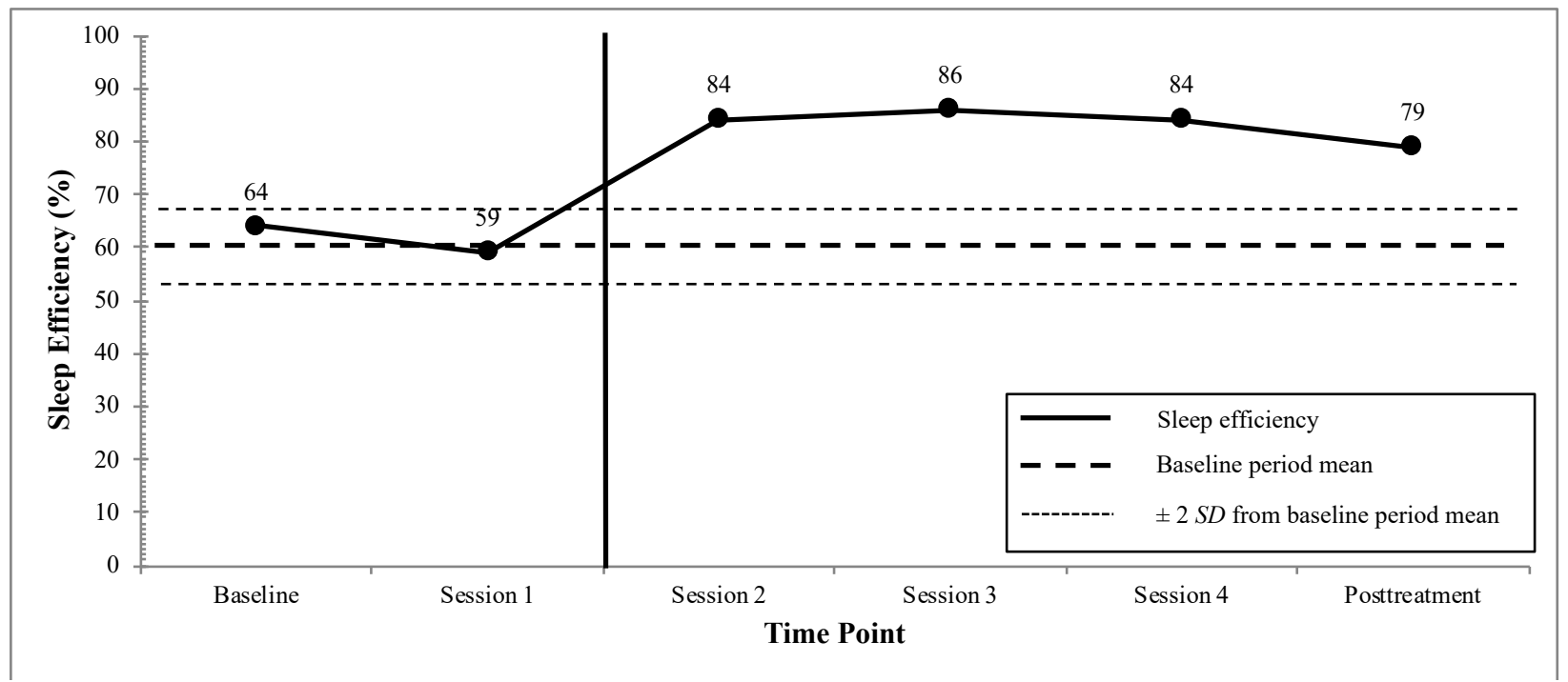

Figure 25a. Participant P10 two-week mean sleep efficiency with baseline period mean $\pm 2 S D$ plotted.

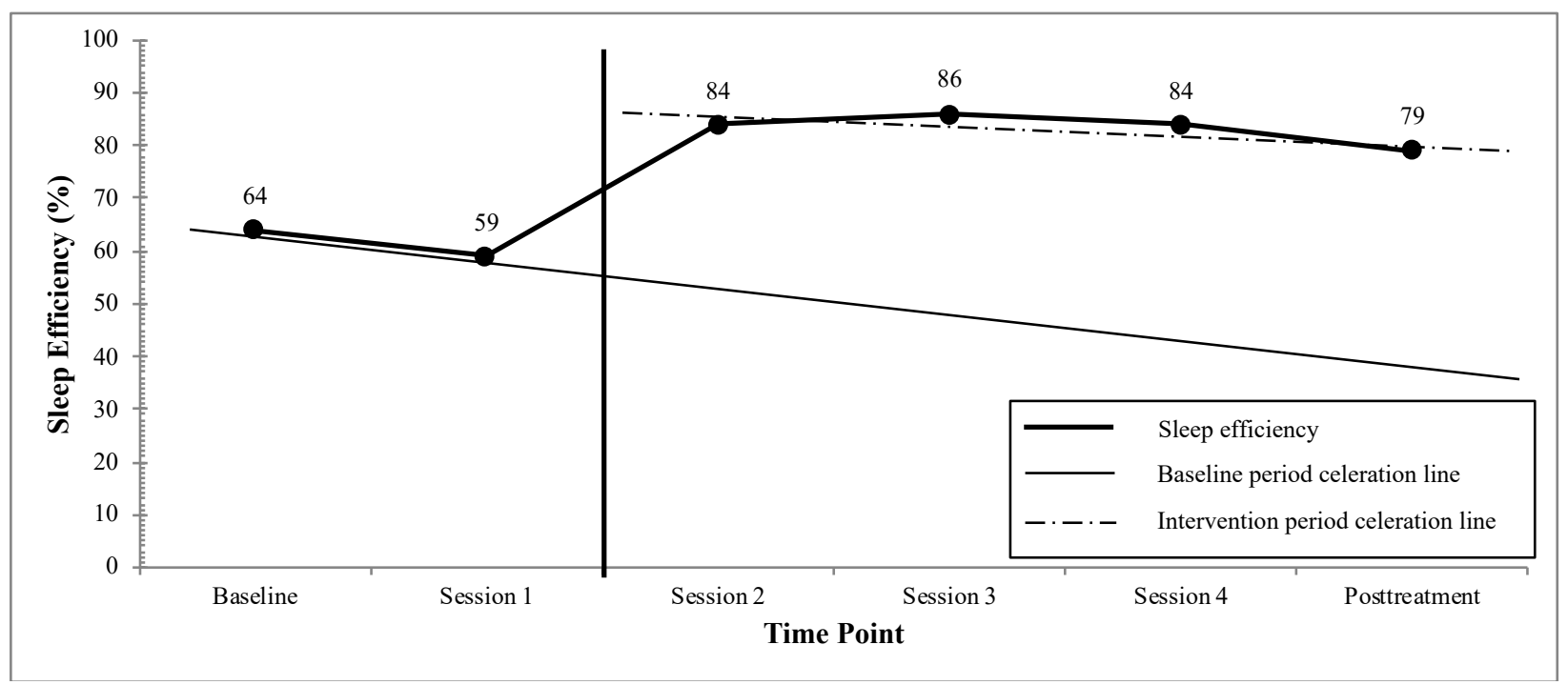

Figure 25b. Participant P10 two-week mean sleep efficiency with baseline and intervention period celeration lines plotted. Celeration lines indicate slope of change during each period, with the baseline celeration line extended into the intervention period for comparison. 
Hypothesis 3: Total wake time. Two-week mean total wake time reduced from 183.1 minutes $(S D=87.6)$ at baseline to 44.0 minutes $(S D=25.7)$ at posttreatment. Cohen's $d$, based on the $S D$ of baseline total wake time was $d=1.59$, indicating a large effect of treatment. The number of data points analyzed using visual inspection ranged from five to seven per participant. All participants had at least two data points to serve as baseline comparison prior to receiving CBT-I. With regard to magnitude of change, visual inspection of total wake time for each participant relative to their respective baseline period $M$ and $\pm 2 S D$ revealed that for all but one participant (P9), total wake time for at least the final two time points was $>2 S D$ below the baseline period mean, which is indicative of reliable change. For participant $\mathrm{P} 9$, total wake time at just the final time point was $>2 S D$ below the baseline period mean. With regard to latency of change, a sustained mean total wake time $>2 S D$ below the baseline period mean was observed after just one session for seven participants, after two sessions for one participant, after three sessions for one participant, and not at all for one participant. Mean total wake time for the sample decreased $>2 S D$ below the baseline mean after just one session.

With regard to trend, visual inspection of celeration lines representing slope of total wake time over time revealed a steeper downward slope in the intervention period relative to the baseline period for only one participant (P4). A floor effect was noted here, whereby slopes in the intervention period flattened out as total wake time decreased toward the minimum possible value of zero. Therefore, visual analysis of celeration lines was not an effective visual aid for determining reliable change in total wake time. Reliable change in total wake time based on magnitude of change was identified for nine of the ten participants. Figure 26 presents the sample mean total wake time at each time point, accompanied by visual aids, and change in total wake time for each participant is presented in Figures 27-36. 


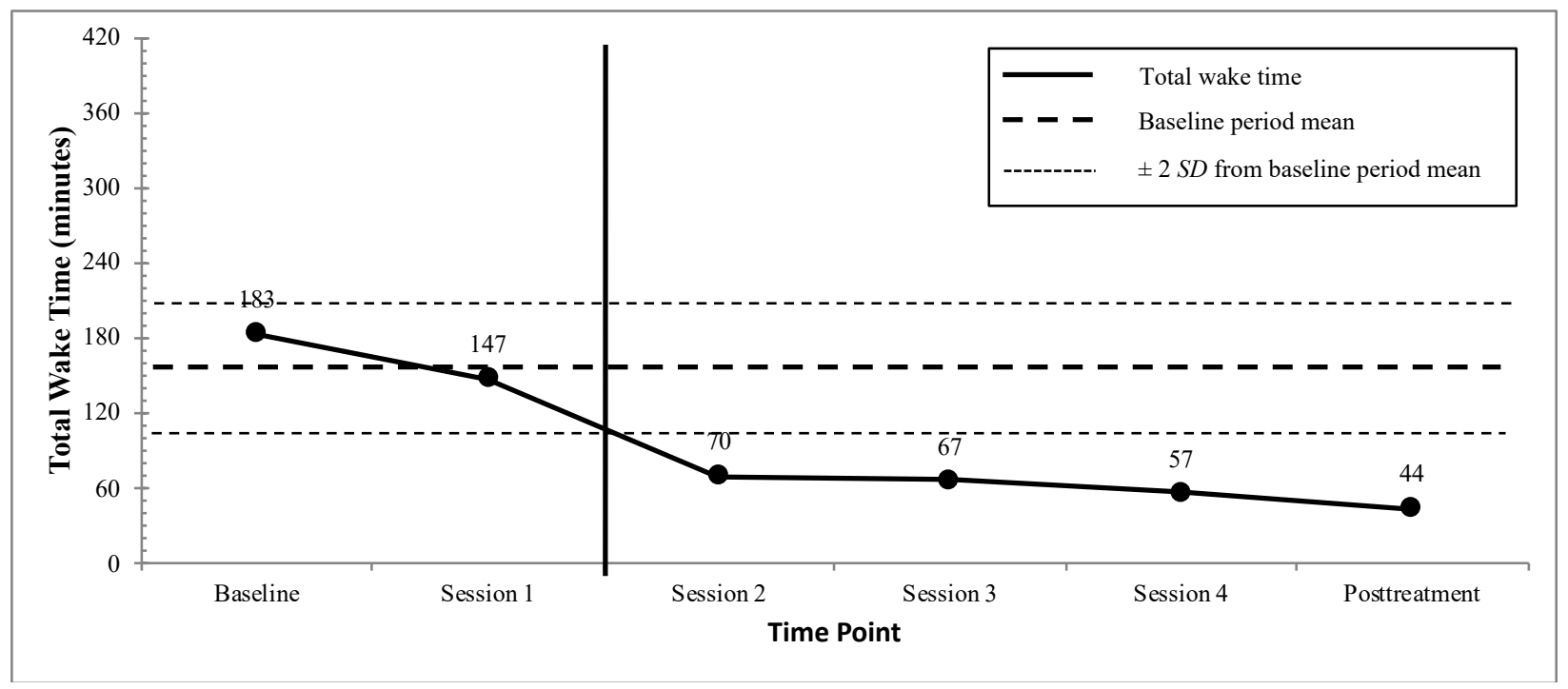

Figure 26a. Two-week mean total wake time with baseline period mean $\pm 2 S D$ plotted, $n=10$.

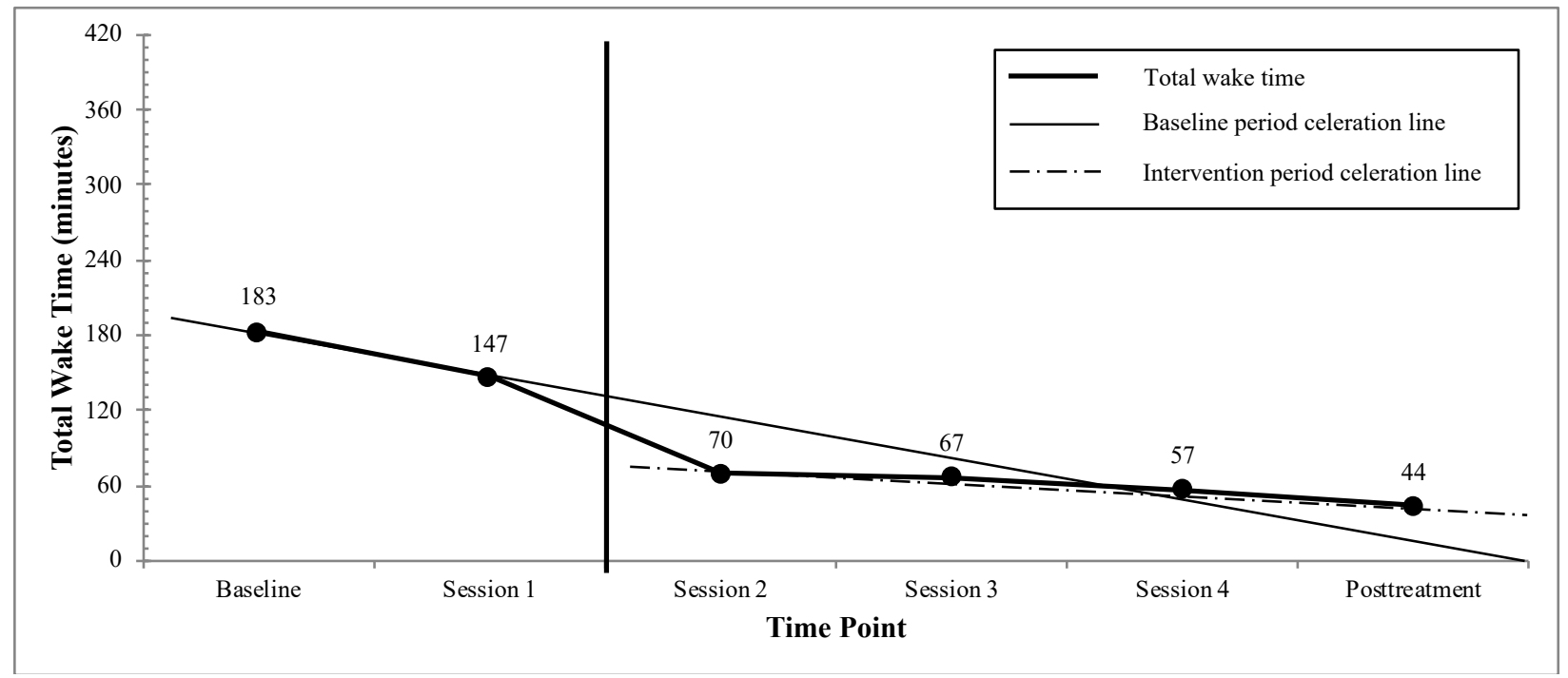

Figure 26b. Two-week mean total wake time with baseline and intervention period celeration lines plotted. Celeration lines indicate slope of change during each period, with the baseline celeration line extended into the intervention period for comparison, $n=10$. 


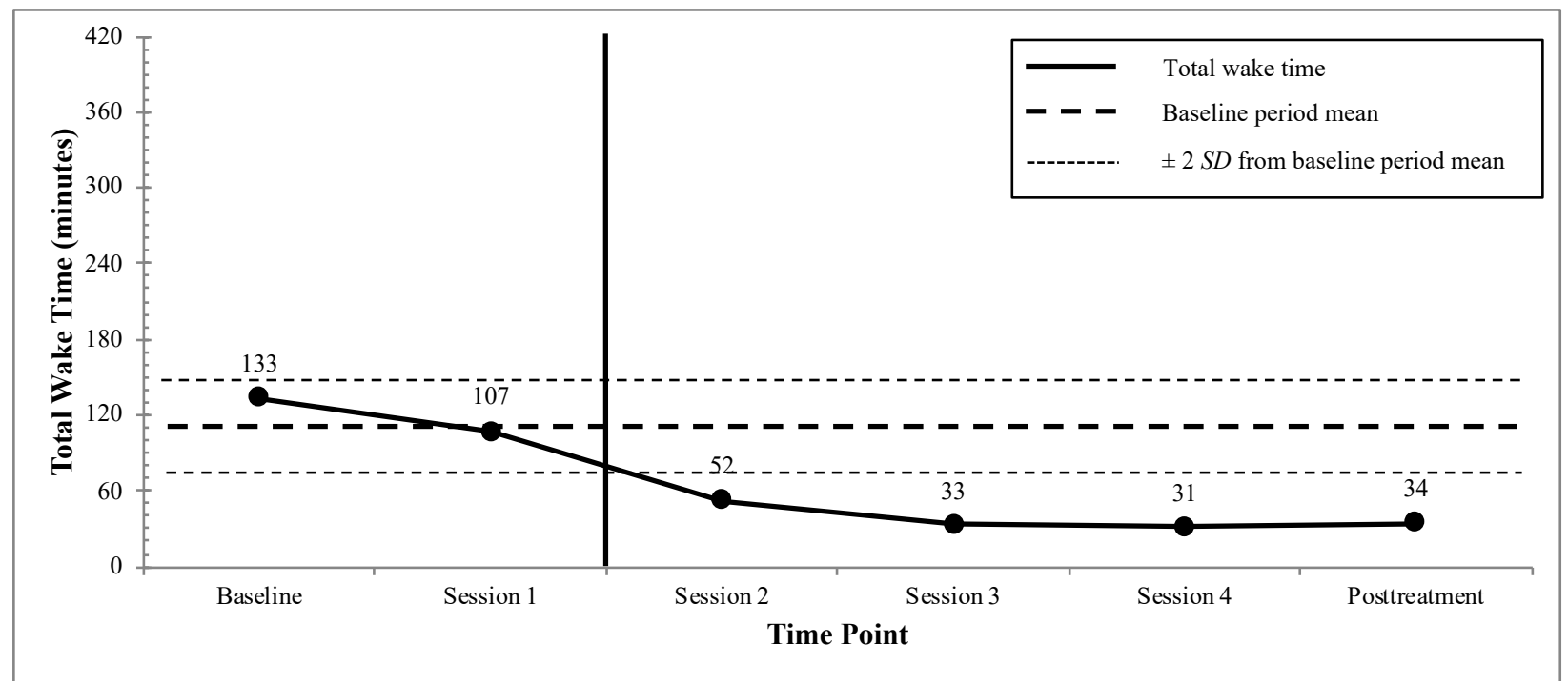

Figure 27a. Participant P1 two-week mean total wake time with baseline period mean $\pm 2 S D$ plotted.

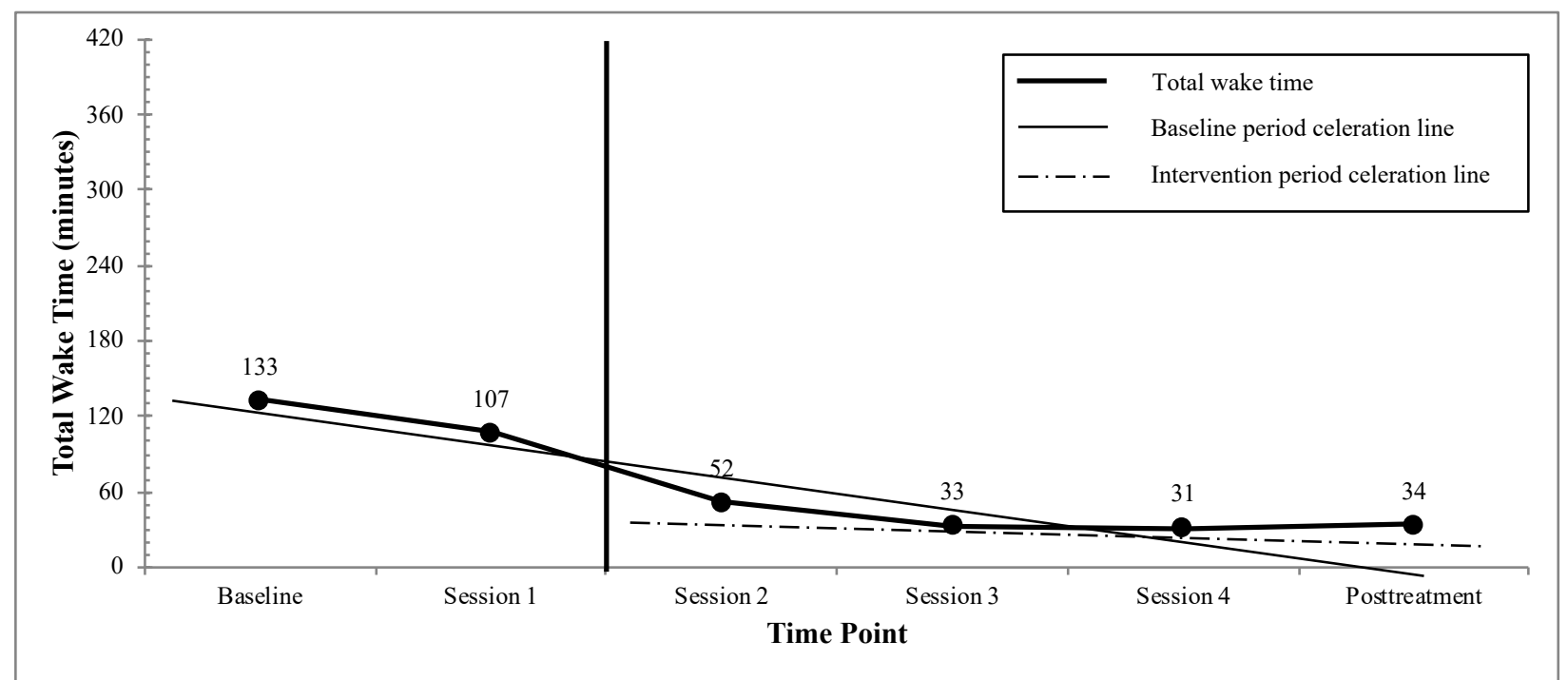

Figure 27b. Participant P1 two-week mean total wake time with baseline and intervention period celeration lines plotted. Celeration lines indicate slope of change during each period, with the baseline celeration line extended into the intervention period for comparison. 


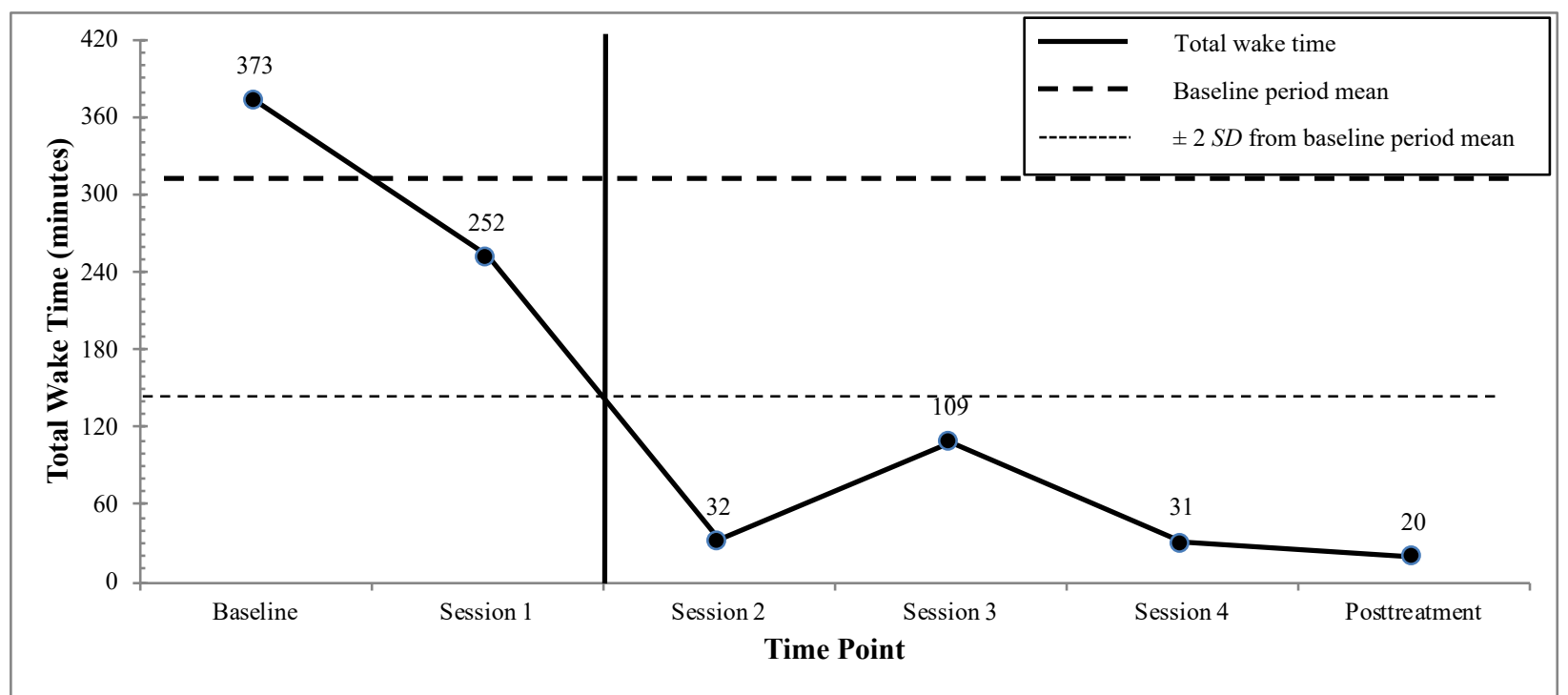

Figure 28a. Participant P2 two-week mean total wake time with baseline period mean $\pm 2 S D$ plotted.

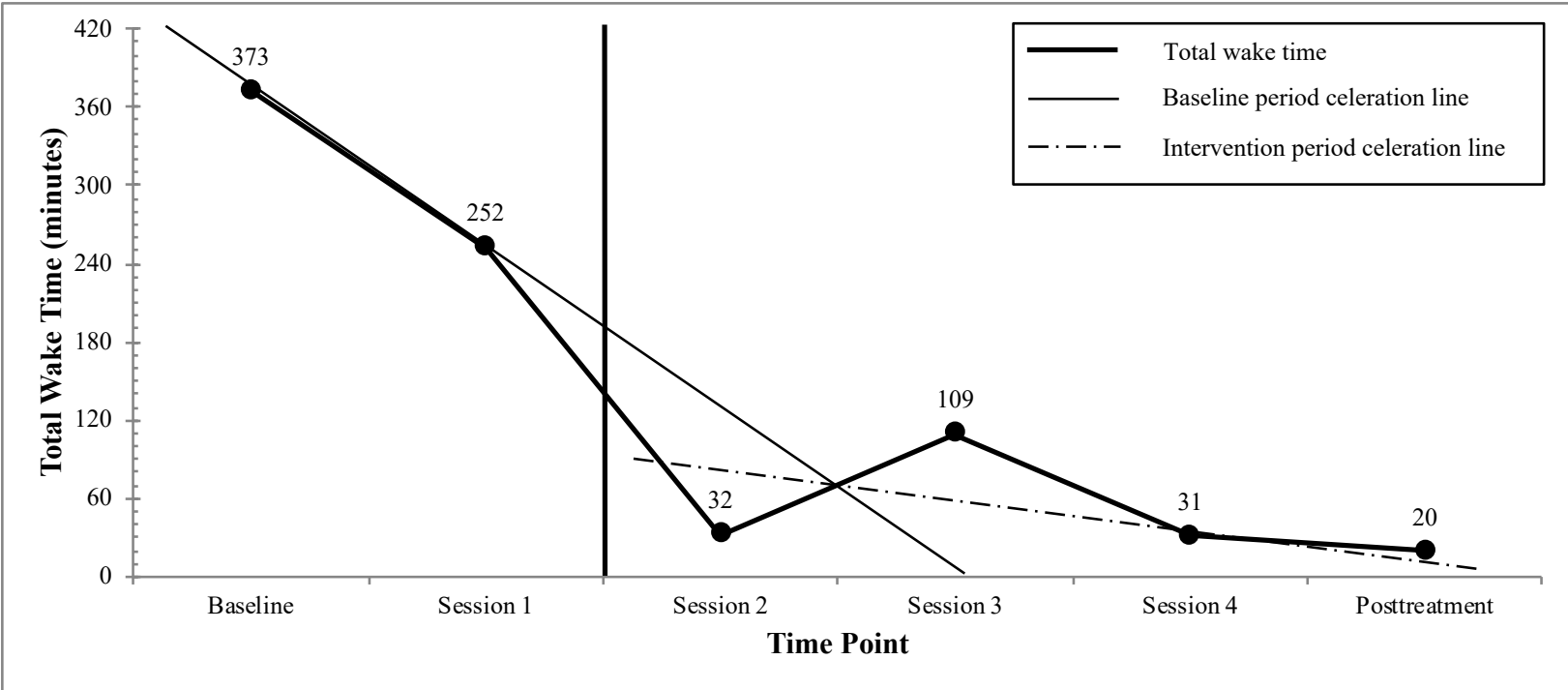

Figure 28b. Participant P2 two-week mean total wake time with baseline and intervention period celeration lines plotted. Celeration lines indicate slope of change during each period, with the baseline celeration line extended into the intervention period for comparison. 


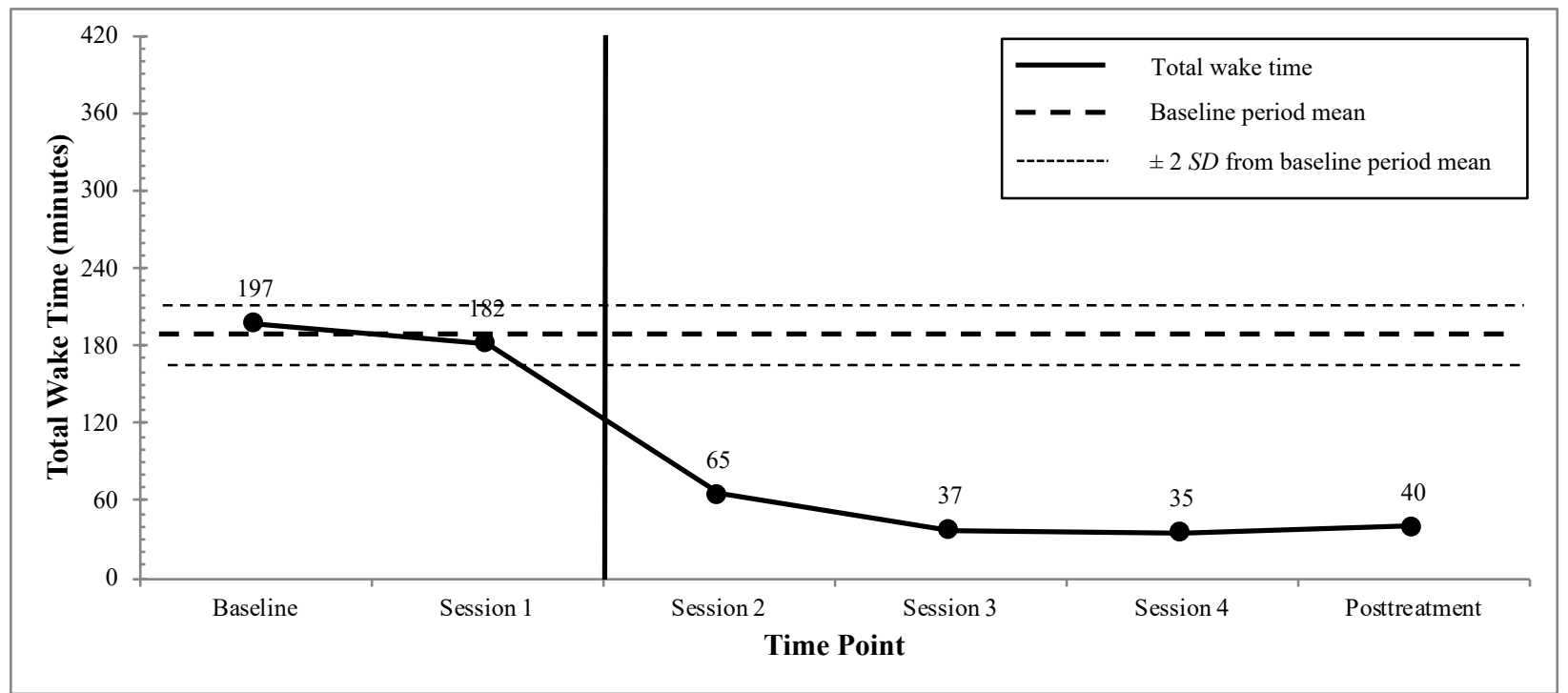

Figure 29a. Participant P3 two-week mean total wake time with baseline period mean $\pm 2 S D$ plotted.

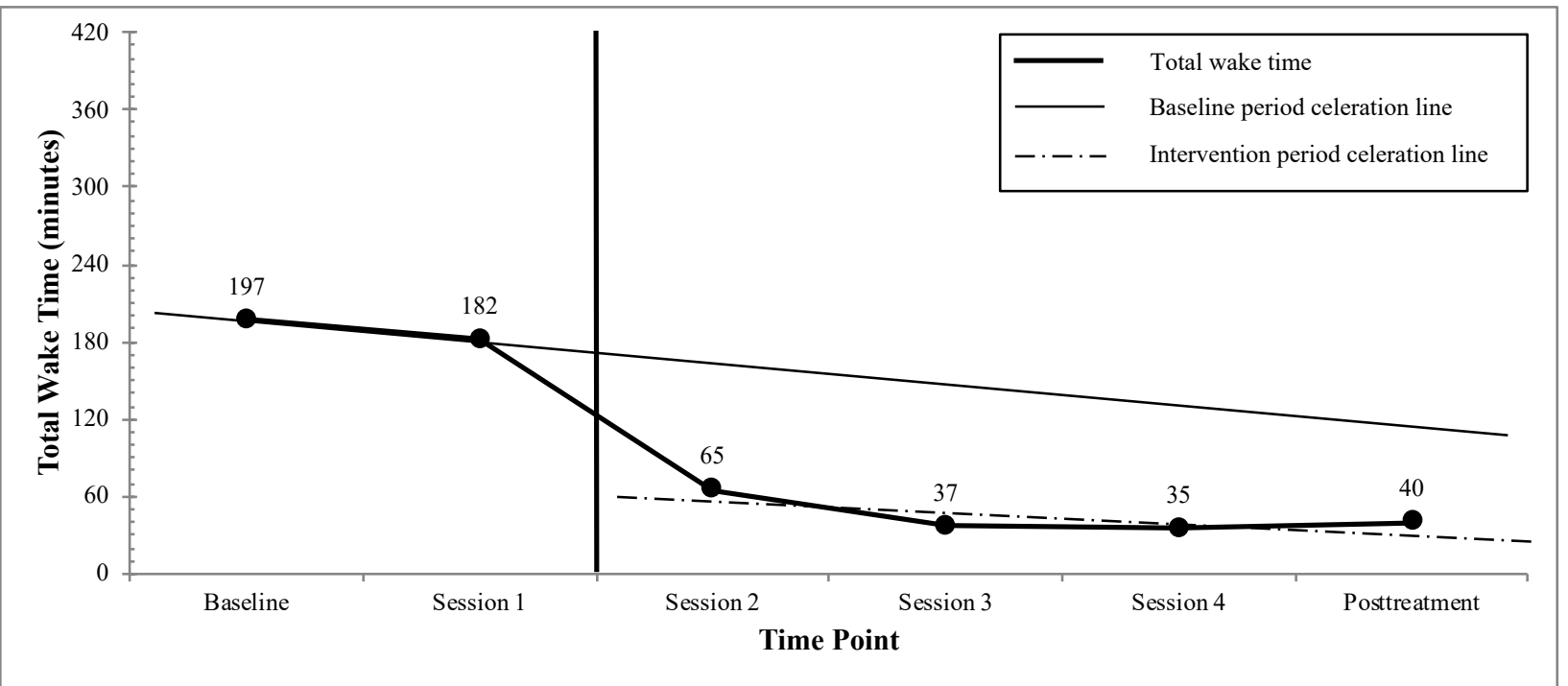

Figure 29b. Participant P3 two-week mean total wake time with baseline and intervention period celeration lines plotted. Celeration lines indicate slope of change during each period, with the baseline celeration line extended into the intervention period for comparison. 


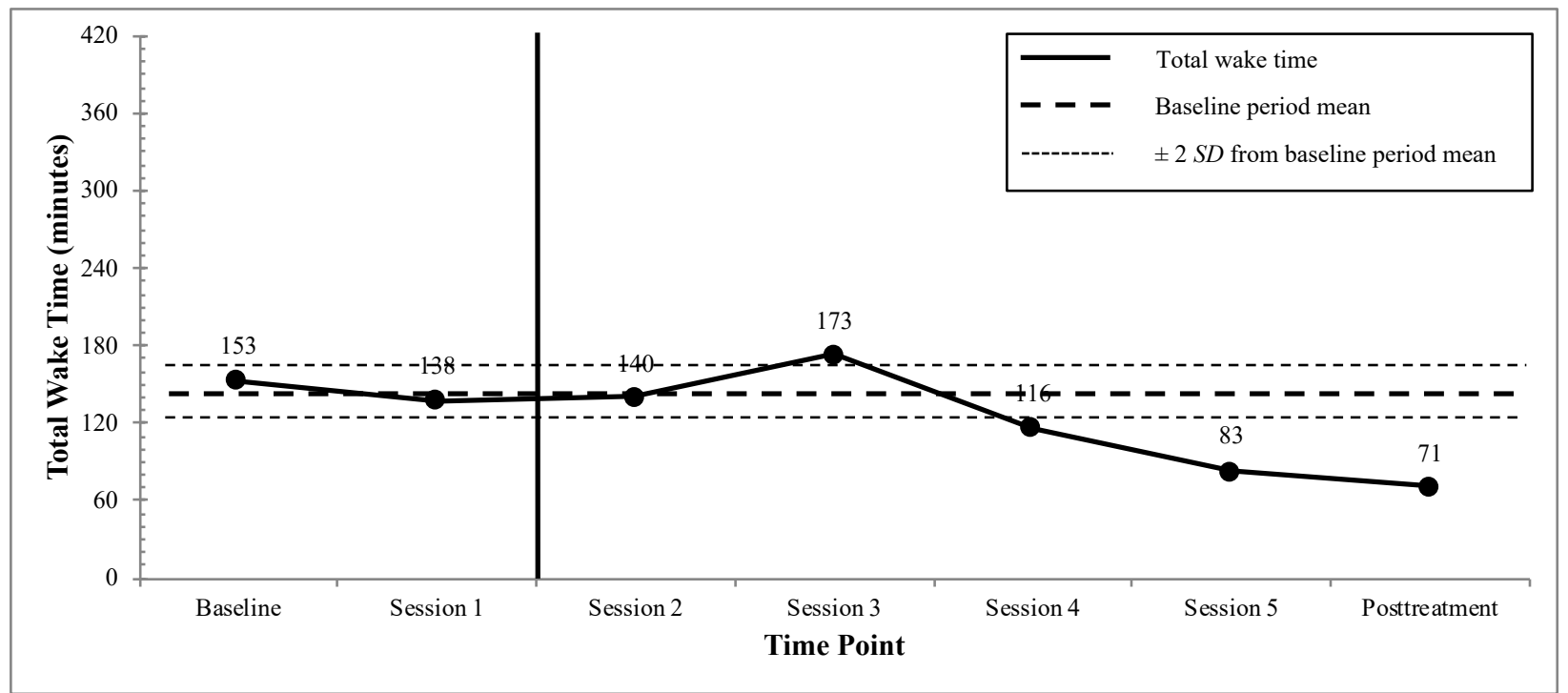

Figure 30a. Participant P4 two-week mean total wake time with baseline period mean $\pm 2 S D$ plotted.

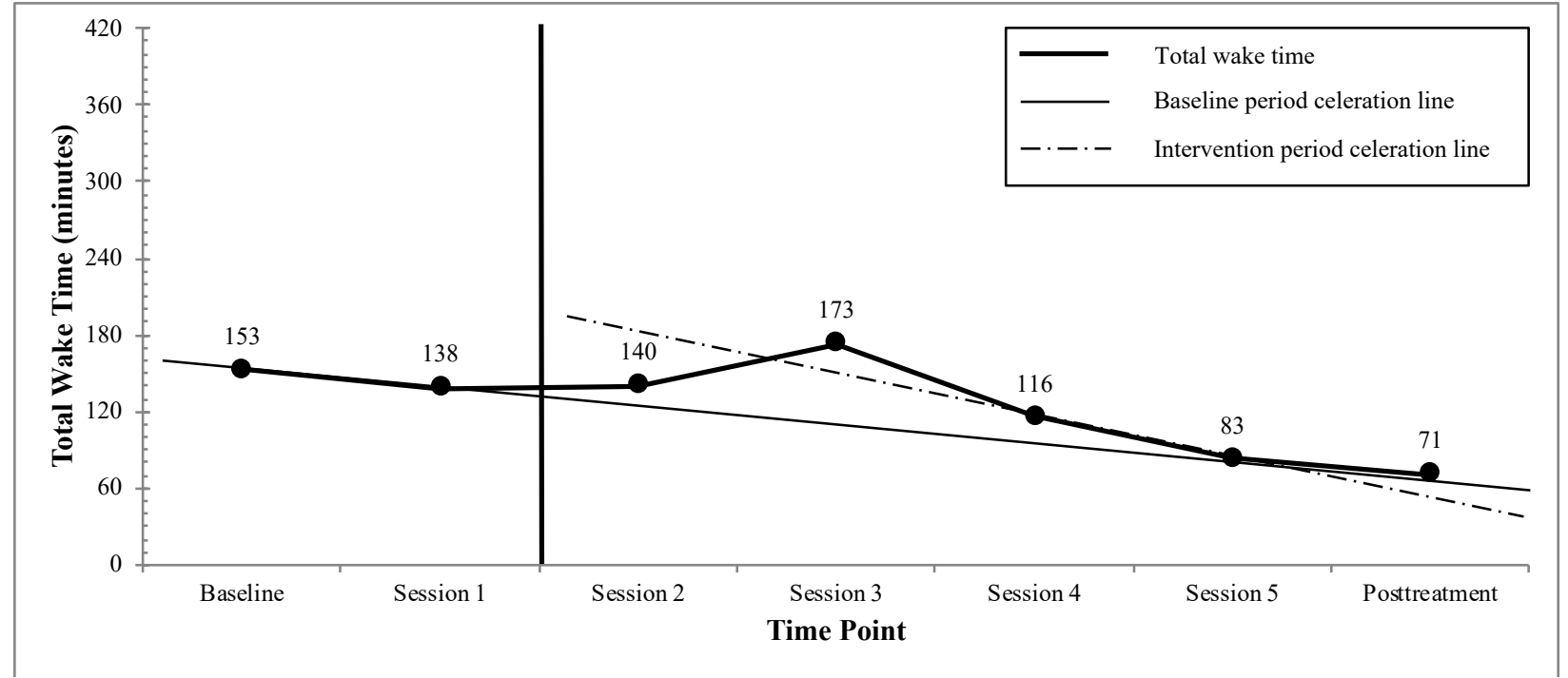

Figure 30b. Participant P4 two-week mean total wake time with baseline and intervention period celeration lines plotted. Celeration lines indicate slope of change during each period, with the baseline celeration line extended into the intervention period for comparison. 


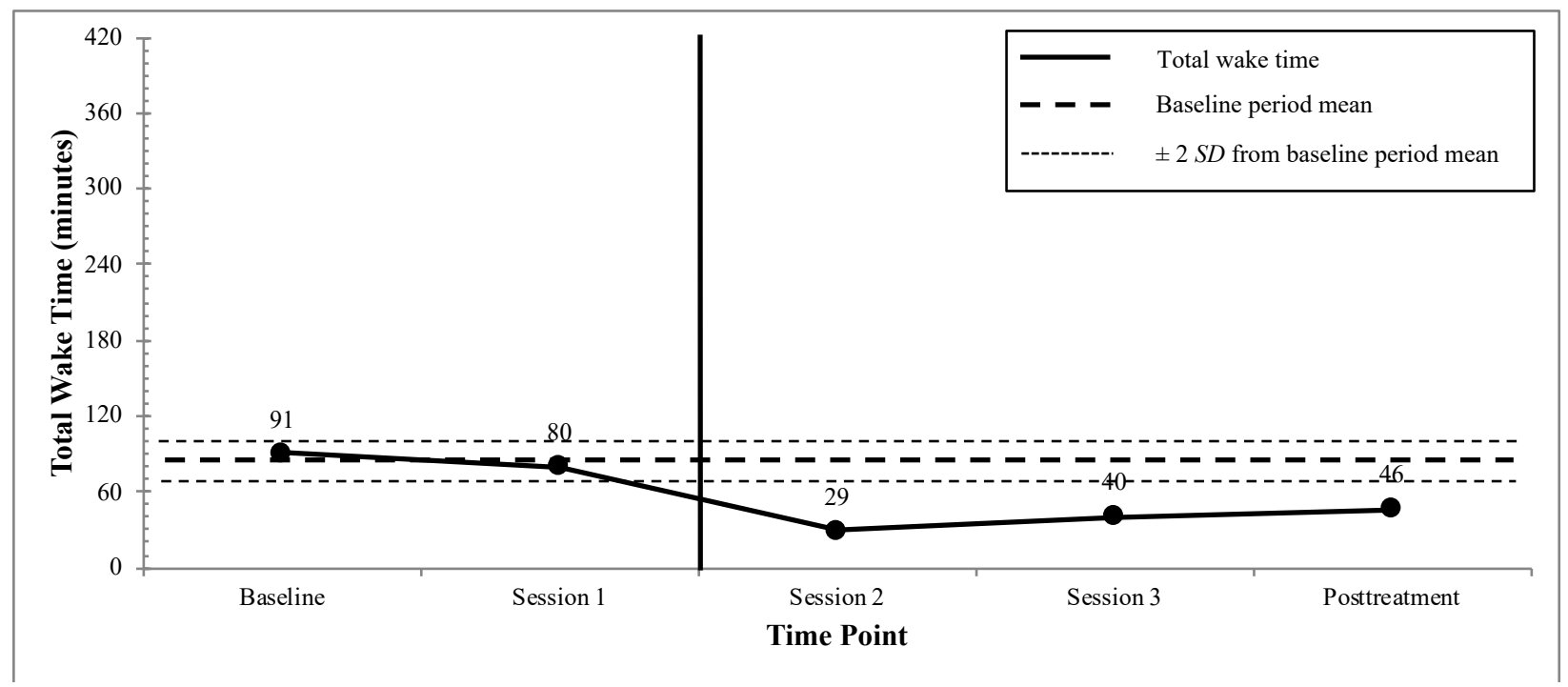

Figure 31a. Participant P5 two-week mean total wake time with baseline period mean $\pm 2 S D$ plotted.

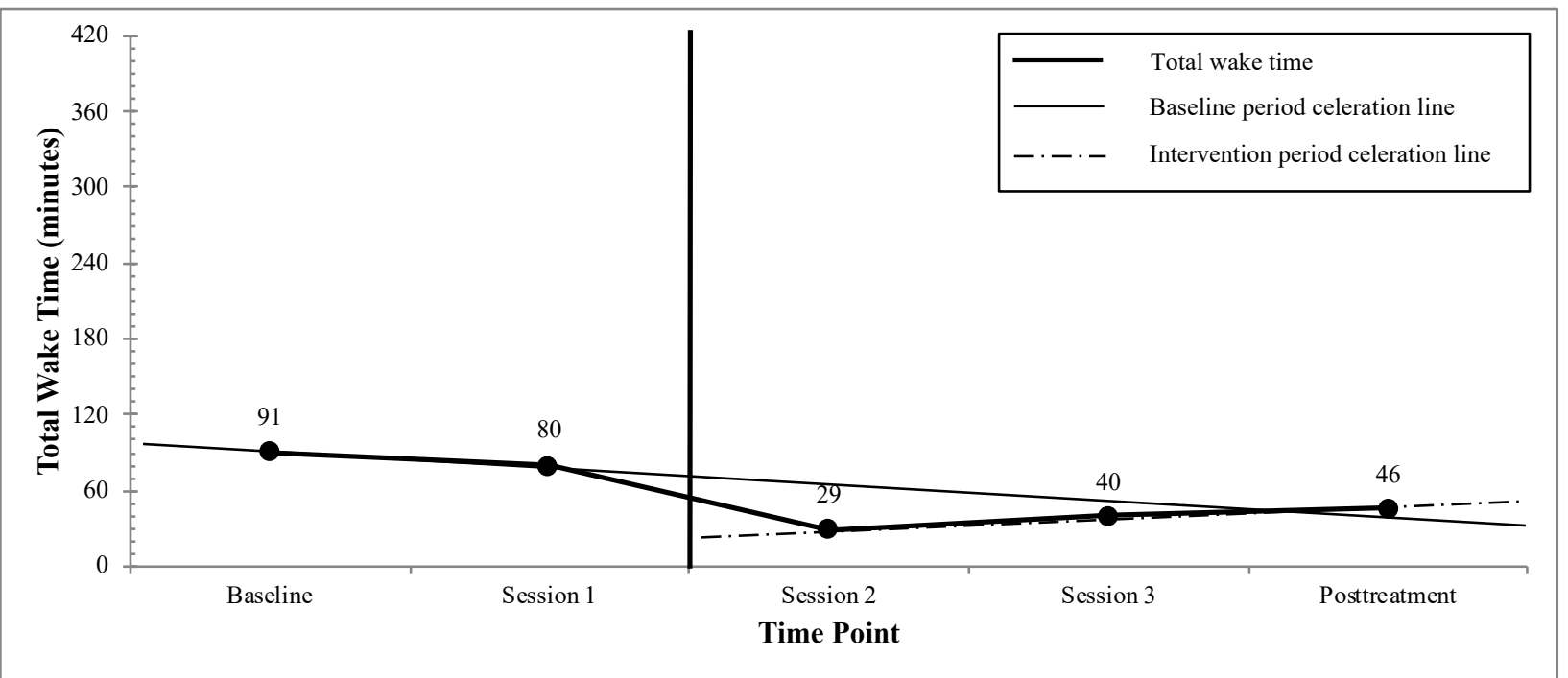

Figure 31b. Participant P5 two-week mean total wake time with baseline and intervention period celeration lines plotted. Celeration lines indicate slope of change during each period, with the baseline celeration line extended into the intervention period for comparison. 


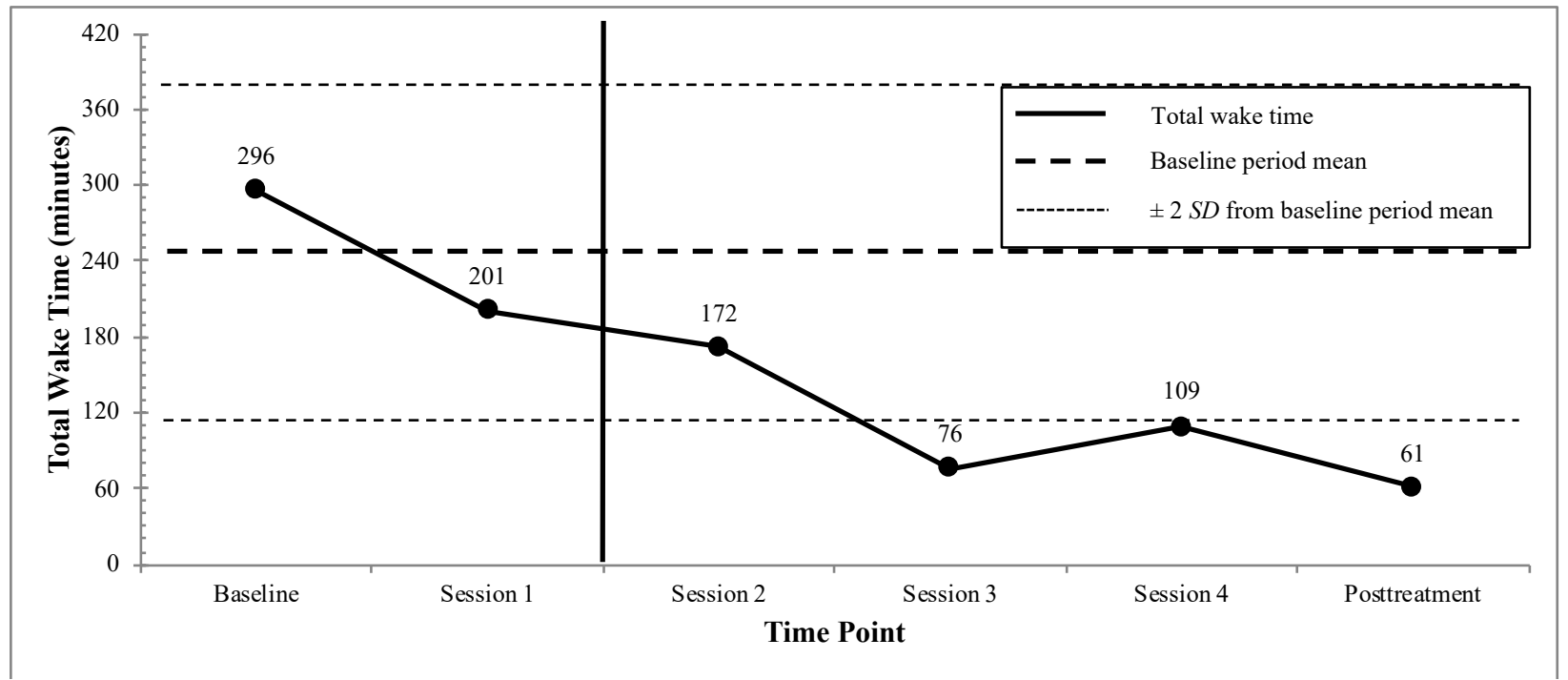

Figure 32a. Participant P6 two-week mean total wake time with baseline period mean $\pm 2 S D$ plotted.

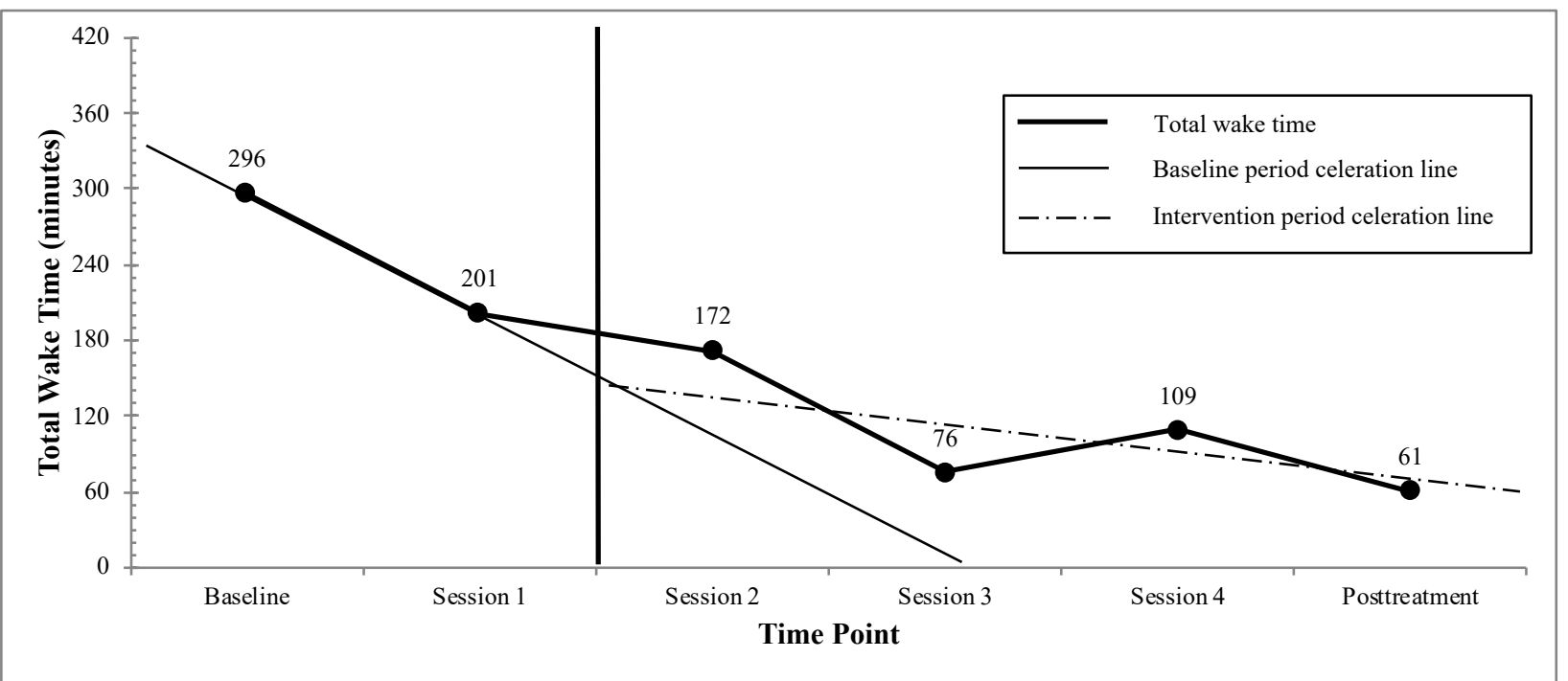

Figure 32b. Participant P6 two-week mean total wake time with baseline and intervention period celeration lines plotted. Celeration lines indicate slope of change during each period, with the baseline celeration line extended into the intervention period for comparison. 


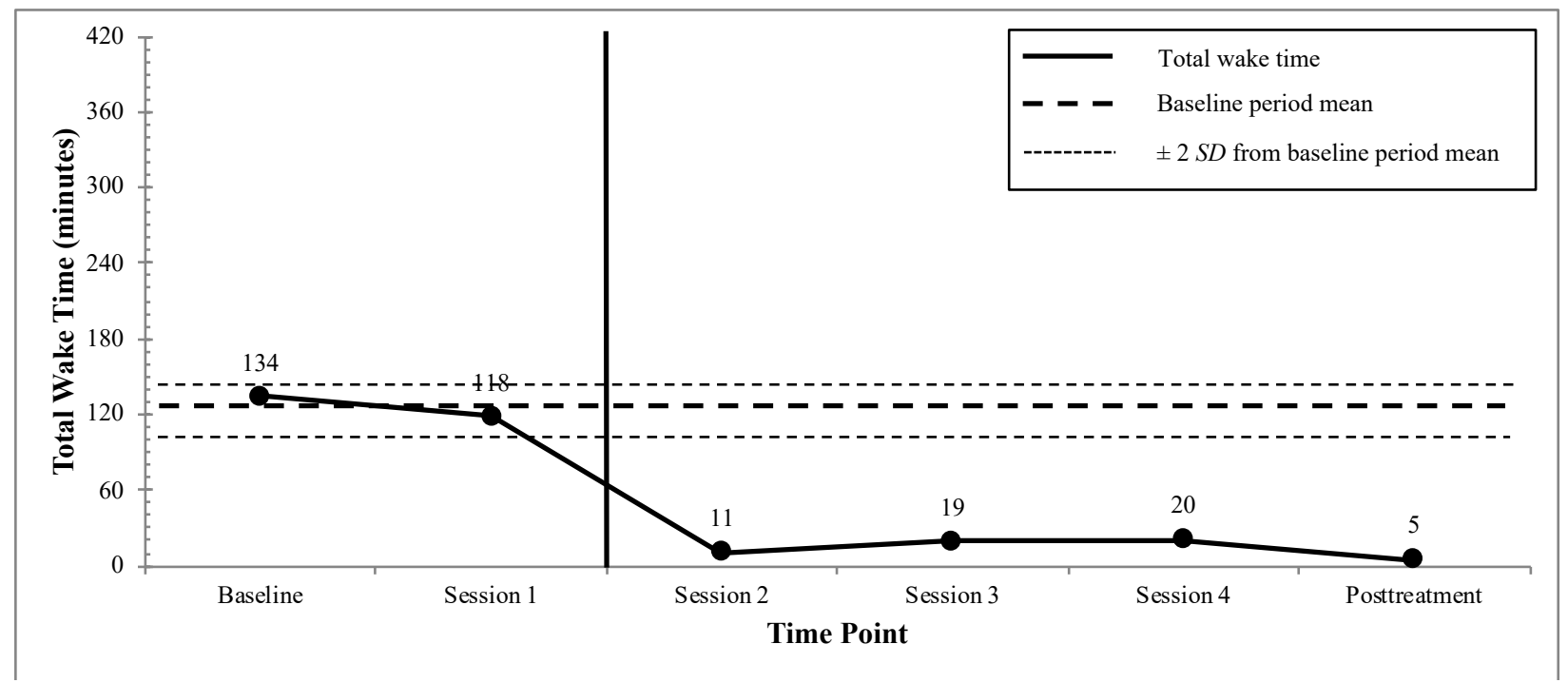

Figure 33a. Participant P7 two-week mean total wake time with baseline period mean $\pm 2 S D$ plotted.

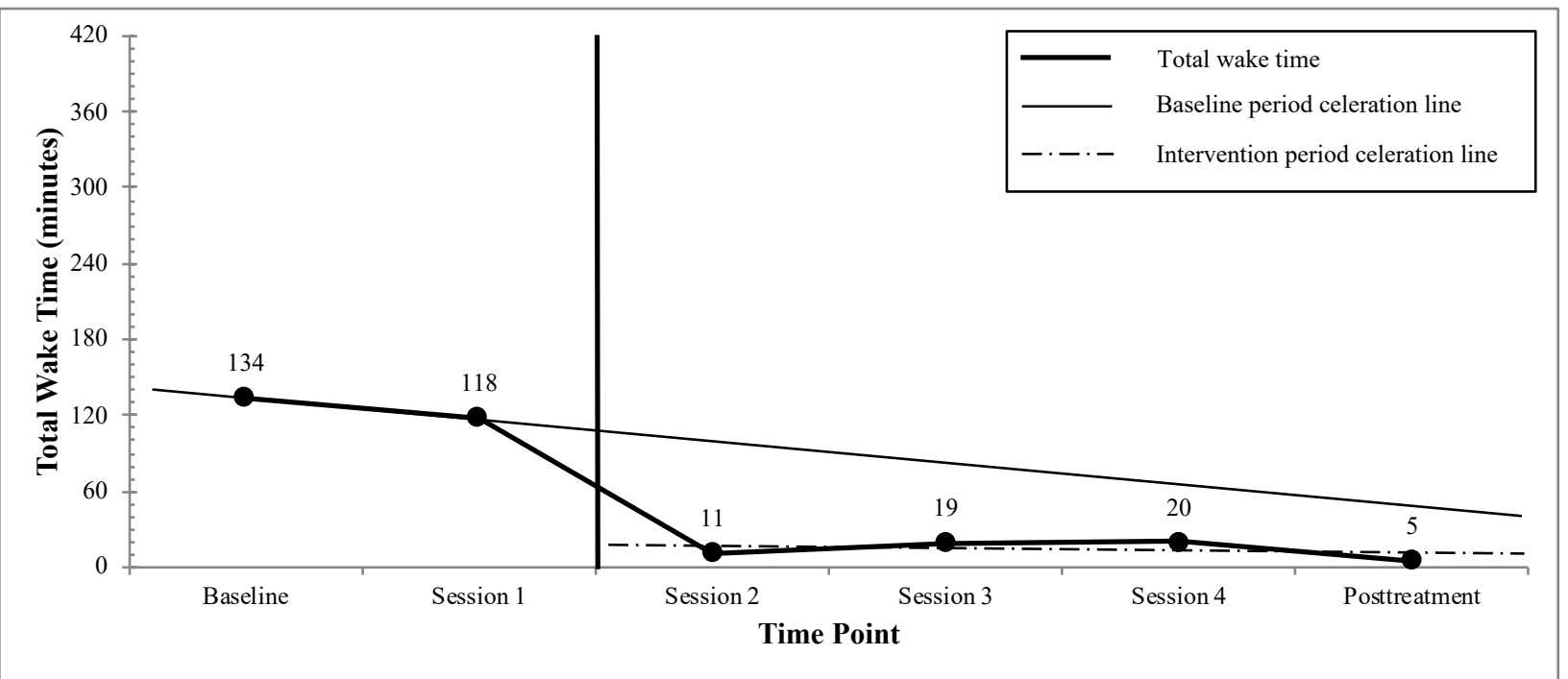

Figure 33b. Participant P7 two-week mean total wake time with baseline and intervention period celeration lines plotted. Celeration lines indicate slope of change during each period, with the baseline celeration line extended into the intervention period for comparison. 


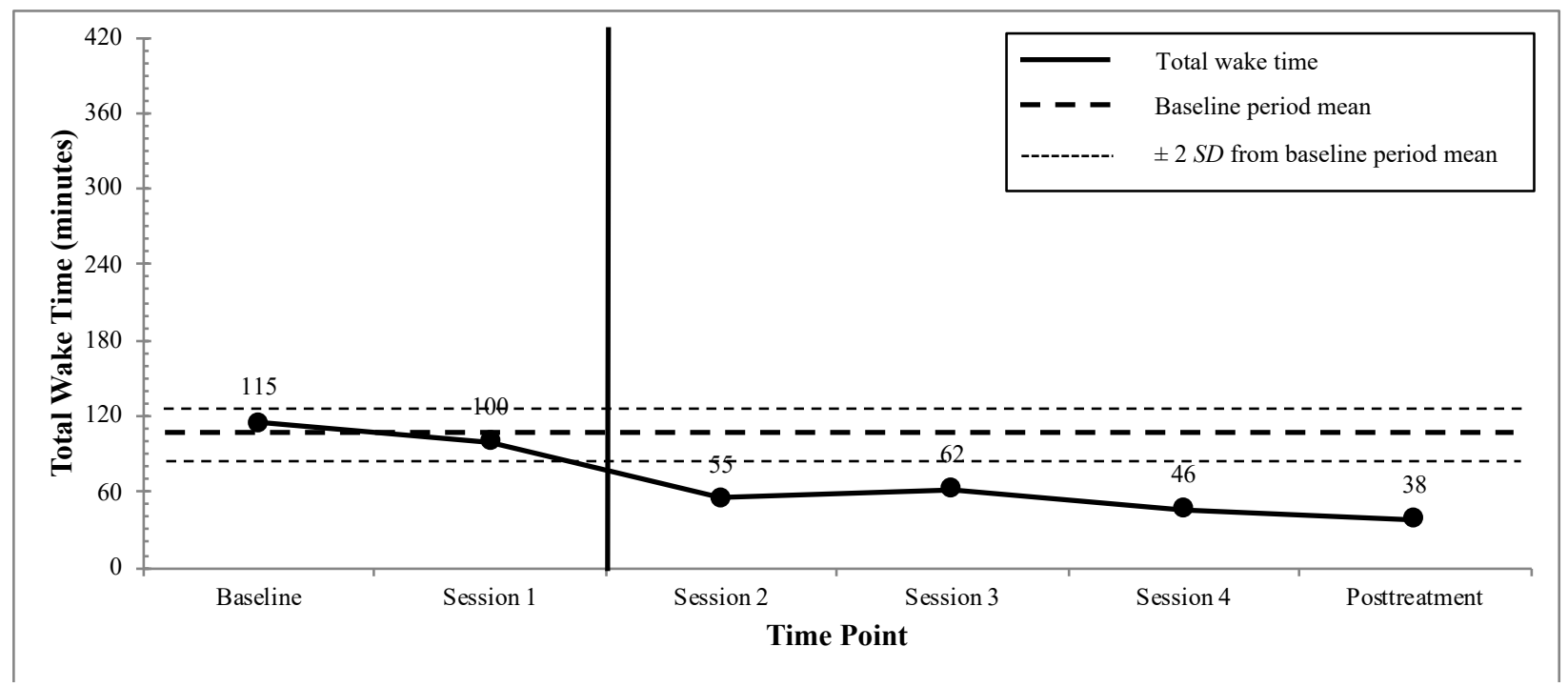

Figure 34a. Participant P8 two-week mean total wake time with baseline period mean $\pm 2 S D$ plotted.

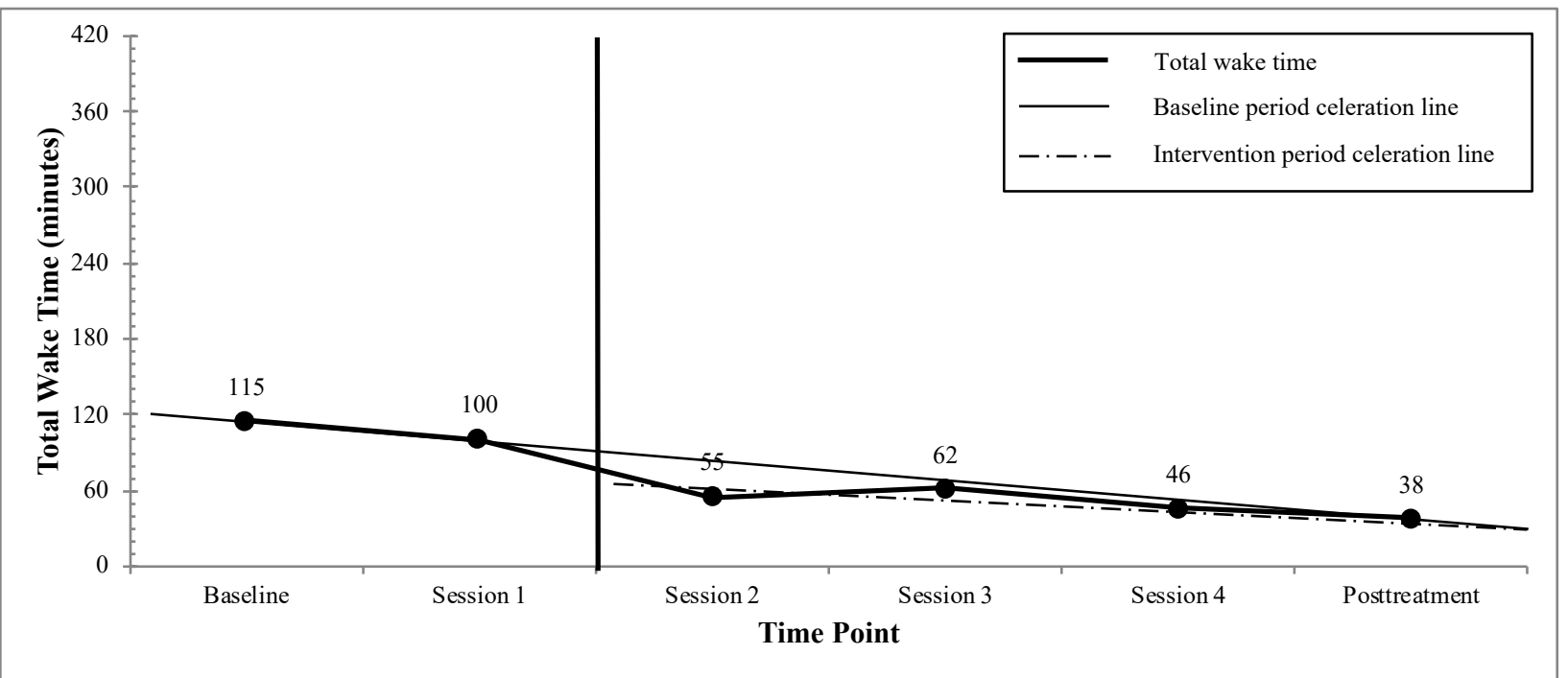

Figure 34b. Participant P8 two-week mean total wake time with baseline and intervention period celeration lines plotted. Celeration lines indicate slope of change during each period, with the baseline celeration line extended into the intervention period for comparison. 


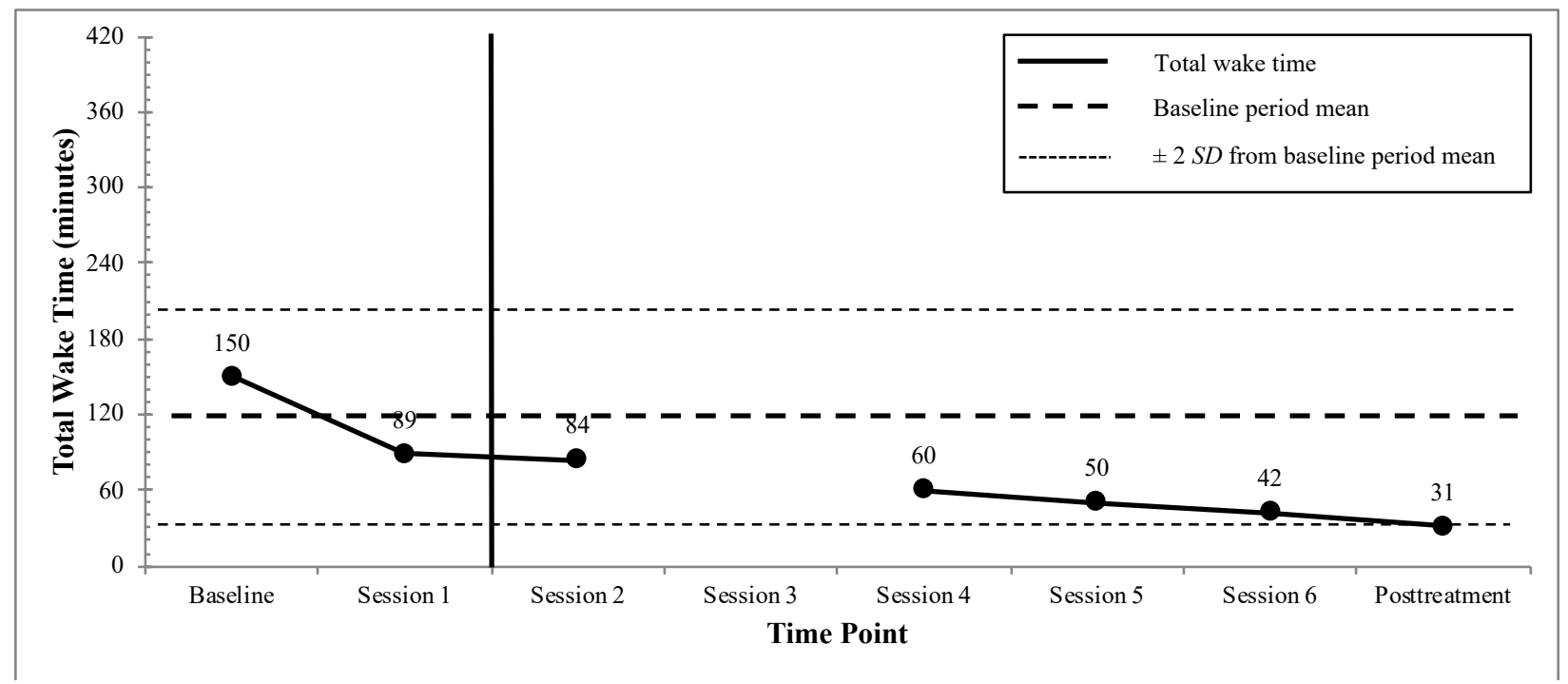

Figure 35a. Participant P9 two-week mean total wake time with baseline period mean $\pm 2 S D$ plotted.

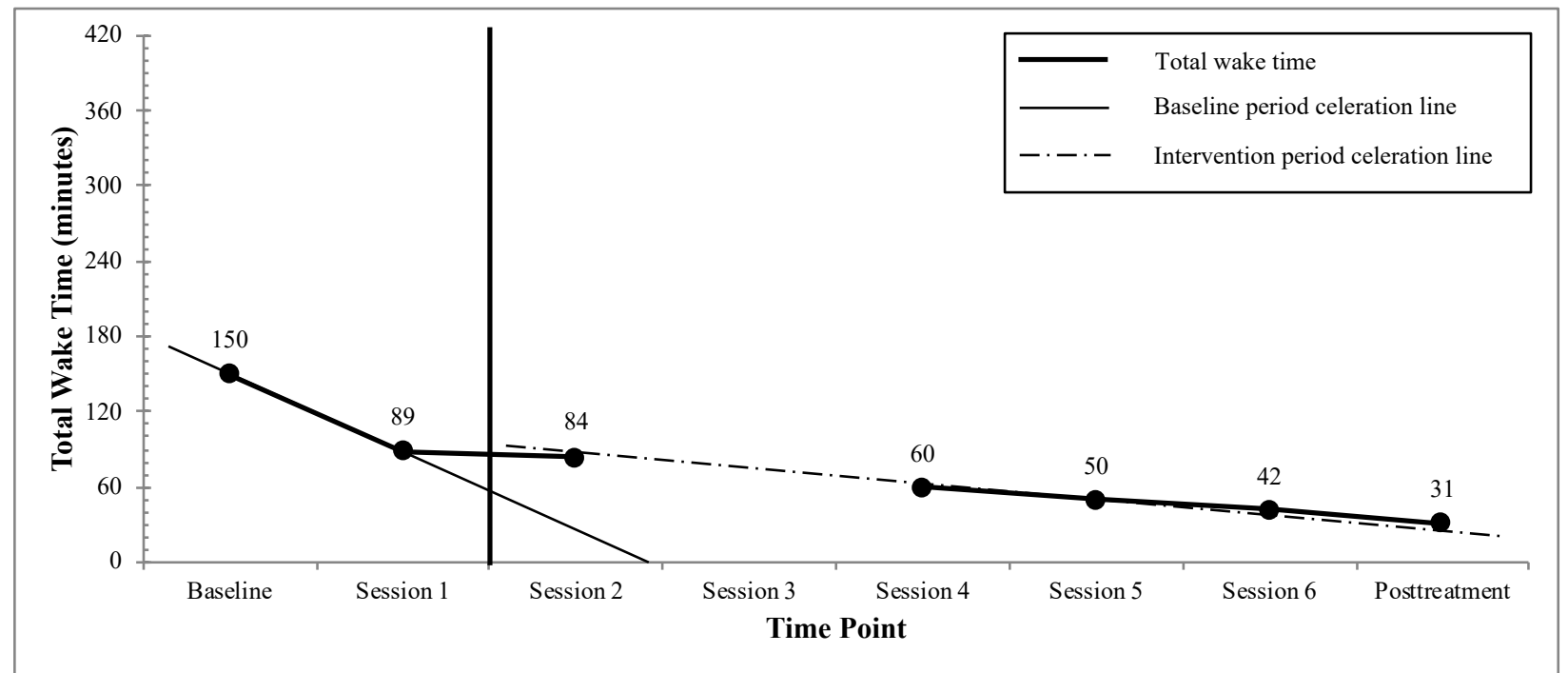

Figure 35b. Participant P9 two-week mean total wake time with baseline and intervention period celeration lines plotted. Celeration lines indicate slope of change during each period, with the baseline celeration line extended into the intervention period for comparison. 


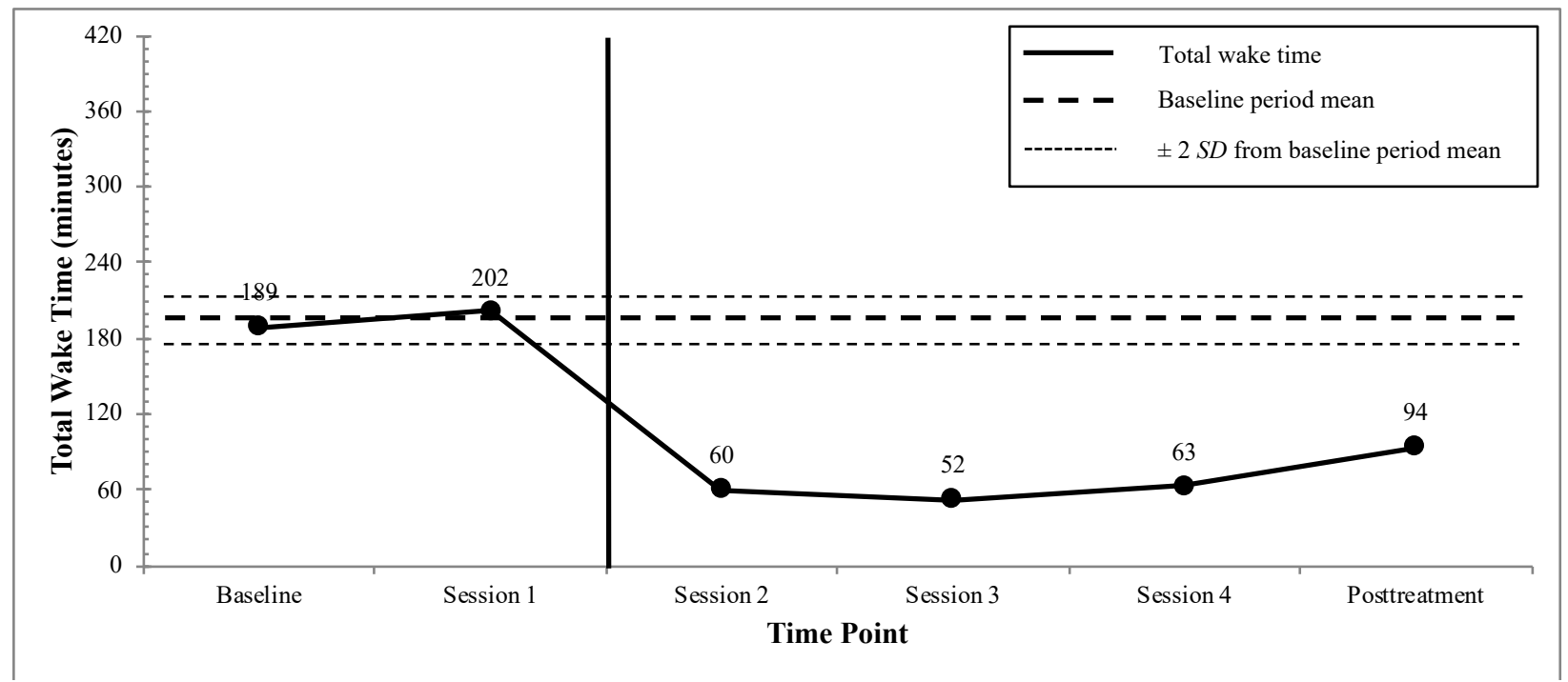

Figure 36a. Participant P10 two-week mean total wake time with baseline period mean $\pm 2 S D$ plotted.

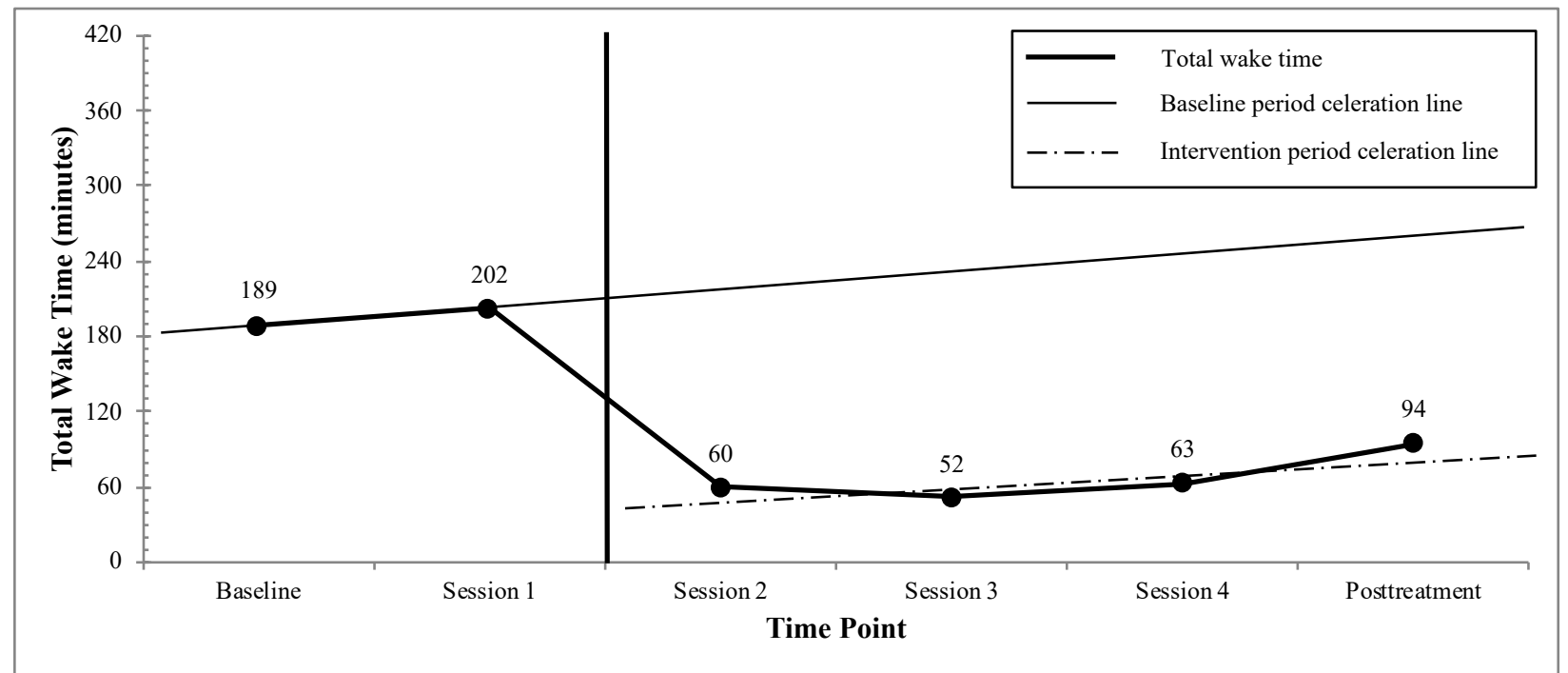

Figure 36b. Participant P10 two-week mean total wake time with baseline and intervention period celeration lines plotted. Celeration lines indicate slope of change during each period, with the baseline celeration line extended into the intervention period for comparison. 
Exploratory paired samples $t$-tests applying bias-corrected bootstrapping to account for non-normally distributed data demonstrated that total wake time significantly reduced from baseline $(M=183.1, S D=87.6)$ to posttreatment $(M=44.0, S D=25.7), t(9)=4.81, p=.001$, bias-corrected 95\% CI $[93.72,196.56]$. The third hypothesis, that participants would experience a significant reduction in total wake time, was supported using multiple analytic approaches, including visual inspection of magnitude of change and statistical significance testing.

Hypothesis 4: HIV viral load. Given that all but one participant reported having an undetectable viral load at baseline, and the remaining participant was awaiting blood test results, this variable was not analyzed. At posttreatment, all participants reported having an undetectable viral load. The fourth hypothesis, that participants would experience a reduction in viral load for participants with a detectable viral load at baseline, was not tested due to the limited amount of data.

Hypothesis 5: CD4 count. Mean CD4 count increased from 620.9 cells $/ \mu \mathrm{L}^{3}(S D=253.9)$ at baseline to 687.2 cells $/ \mu \mathrm{L}^{3}(S D=303.2)$ at posttreatment. Cohen's $d$, based on the $S D$ of baseline scores was $d=-0.26$, indicating a small effect of treatment on CD4 count. Both the baseline and posttreatment CD4 levels were within the normal range of 500 cells $/ \mu \mathrm{L}^{3}$ to 1,600 cells $/ \mu \mathrm{L}^{3}$ for individuals with healthy immune functioning (Public Health Agency of Canada, 2016), therefore this change was not clinically significant. Exploratory paired samples $t$-tests applying bias-corrected bootstrapping to account for non-normally distributed data demonstrated that the increase in CD4 count was not statistically significant, $t(7),=-1.76, p=.121$, biascorrected 95\% CI [-197.2, 14.4]. Data for only eight participants were used for this calculation due to missing posttreatment data for two participants. The fifth hypothesis, that participants would experience an increase in CD4 count, was not supported. While there was a small increase 
in CD4 count from baseline to posttreatment, this change was neither clinically nor statistically significant.

Hypothesis 6: $\boldsymbol{c A R T}$ adherence. According to self-report on the SRSI, the frequency of participants reporting their cART adherence as excellent increased from $70 \%$ at baseline to $100 \%$ at posttreatment. However, according to self-report on the SMAQ, a composite measure of cART adherence that categorizes participants as either adherent or nonadherent, the frequency of cART-adherent participants decreased from $40 \%$ at baseline to $30 \%$ at posttreatment. Of the participants that were categorized as nonadherent, two received nonadherent designations due to not answering the question "How many days did you miss taking all your medication during the past 3 months? Please enter the number of days below". Of the remaining five participants who received nonadherent designations, four reported missing their dose 1-3 days during the past three months, and the fifth participant reported not missing any days over the past three months, but did respond "yes" to the question "Do you ever forget to take your medication?". Missing data on the composite measure precludes useful interpretation. Furthermore, four of the participants who were classified as being nonadherent on the composite measure reported very infrequent nonadherence. The sixth hypothesis, that participants would experience improved cART adherence, was partially supported. Results from the single-item measure of adherence supported the hypothesis, whereas results from the composite measure did not.

Exploratory analyses. To examine symptom change intercorrelation, Spearman correlations were calculated on all variables that, at baseline, were correlated with ISI, sleep efficiency, and total wake time. The results are presented in Table 5. Change in ISI was not associated with change in any of the sleep parameters collected using the CSD, but was positively associated with change in GSES, $r=.68, p=.029$, CES-D, $r=.86, p=.002$, and 
Table 5

Spearman Correlations Between Baseline to Posttreatment Symptom Severity Change Scores $(\mathrm{n}=10)$

\begin{tabular}{|c|c|c|c|c|c|c|c|c|c|c|c|c|c|c|c|c|}
\hline Variable & 1 & 2 & 3 & 4 & 5 & 6 & 7 & 8 & 9 & 10 & 11 & 12 & 13 & 14 & 15 & 16 \\
\hline 1. ISI & - & & & & & & & & & & & & & & & \\
\hline 2. $\mathrm{SE}$ & -.32 & - & & & & & & & & & & & & & & \\
\hline 3. TWT & .10 & $-.94 * * *$ & * - & & & & & & & & & & & & & \\
\hline 4. SOL & -.21 & $-.78 * *$ & $.81 * *$ & - & & & & & & & & & & & & \\
\hline 5. NWAK & -.04 & $-.72 *$ & $.79 * *$ & .61 & - & & & & & & & & & & & \\
\hline 6. WASO & .12 & -.50 & $.66^{*}$ & .47 & .33 & - & & & & & & & & & & \\
\hline 7. TWASO & -.08 & -.27 & .19 & .08 & .31 & -.49 & - & & & & & & & & & \\
\hline 8. TST & -.57 & .55 & -.47 & -.07 & -.42 & -.30 & -.09 & - & & & & & & & & \\
\hline 9. TIB & -.29 & -.53 & .59 & $.76^{*}$ & $.67^{*}$ & .31 & .20 & .19 & - & & & & & & & \\
\hline 10. FSS-7 & .23 & .22 & -.41 & -.26 & $-.64 *$ & -.16 & -.14 & -.06 & -.36 & - & & & & & & \\
\hline 11. GSES & $.68^{*}$ & -.28 & .11 & -.13 & -.13 & .28 & .00 & -.42 & -.06 & .62 & - & & & & & \\
\hline 12. SE-S & -.43 & .54 & -.48 & -.31 & -.09 & -.56 & .01 & .18 & -.34 & -.30 & $-.75 * *$ & - & & & & \\
\hline 13. DASS-a & $.74 *$ & -.24 & -.02 & -.14 & -.34 & -.16 & .19 & -.47 & -.46 & .53 & .58 & -.29 & - & & & \\
\hline 14. DASS-s & $.75^{*}$ & -.36 & .14 & -.02 & -.01 & -.04 & -.03 & $-.71 *$ & -.38 & .28 & .34 & -.15 & $.76^{*}$ & - & & \\
\hline 15. CES-D & $.86^{* *}$ & -.09 & -.07 & -.25 & -.38 & .29 & -.42 & -.37 & -.42 & .40 & $.65^{*}$ & -.46 & $.71 *$ & $.64 *$ & - & \\
\hline 16. SF-36-social & -.40 & .24 & -.21 & -.17 & .10 & -.41 & .37 & .44 & .22 & -.02 & -.10 & .08 & -.48 & -.54 & $-.64 *$ & - \\
\hline
\end{tabular}

Note. CES-D = Centre for Epidemiological Studies in Depression Scale; DASS-a = Depression Anxiety Stress Scales-anxiety subscale; DASS-s = Depression Anxiety Stress Scales-stress subscale; FSS-7 = Fatigue Severity Scale (7-item); GSES = Glasgow Sleep Effort Scale; ISI = Insomnia Severity Index; NWAK = number of awakenings; SE = sleep efficiency; SE-S = Self-Efficacy for 
Sleep Scale; SF-36-social = Medical Outcomes Study Short-Form Health Survey-social functioning subscale; SOL $=$ sleep onset latency; TIB = time in bed; TST = total sleep time; TWASO = terminal wake after sleep onset; TWT = total wake time; WASO = wake after sleep onset. 
DASS anxiety, $r=.74, p=.014$, and stress, $r=.75, p=.013$, subscales. Not included in Table 5, change in ISI was negatively associated with change in the SF-36 fatigue/energy subscale, $r=$ $.74, p=.015$, meaning that as insomnia severity decreased, fatigue/energy-related quality of life increased. Change in sleep efficiency was negatively associated with change in the following sleep parameters collected using the CSD: total wake time, $r=-.94, p<.001$, sleep onset latency, $r=-.78, p=.008$, and number of awakenings, $r=-.72, p=.018$. Change in total wake time was positively associated with change in the following sleep parameters: sleep onset latency, $r=.81$, $p=.005$, number of awakenings, $r=.79, p=.006$, and wakefulness after sleep onset, $r=.66, p=$ .038. Not included in Table 5, change in CD4 count was positively associated with change in total sleep time, $r=.71, p=.047$, and change in the SF-36 social functioning subscale, $r=.73, p$ $=.041$. Primary type of cART medication regimen was not associated with change in ISI, $r=$ $.60, p=.07$, sleep efficiency, $r=-.30, p=.40$, or total wake time, $r=.22, p=.54$

Covariates. The $M$ and $S D$ of baseline and posttreatment covariate scores are presented in Table 4, along with Cohen's $d$ effect sizes. Large effects were observed for time in bed, $d=1.88$, number of awakenings, $d=0.99$, wakefulness after sleep onset, $d=0.85$, terminal wakefulness after sleep onset, $d=1.39$, GSES, $d=1.69$, SE-S,$d=-1.21$, DBAS- $16, d=0.94$, and FSS-7, $d=$ 0.82. This represents an expected decrease in time in bed, number of awakenings, wakefulness after sleep onset, time in bed after final awakening, sleep effort, dysfunctional beliefs about sleep, and fatigue. An expected increase in self-efficacy for sleep was also noted. Medium effect sizes were observed for sleep onset latency, $d=0.72$, total sleep time, $d=-0.53$, daytime napping, $d=0.72, d=0.62$, PSAS-13 cognitive arousal subscale, $d=0.53$, and number of bothersome HIV-related symptoms, $d=0.51$. This represents a decrease in sleep onset latency, daytime napping, cognitive arousal, and bothersome HIV-related symptoms, and an increase in 
total sleep time. Small effect sizes were observed for DASS-21 stress subscale, $d=0.49$, CES-D, $d=0.46$, and the following SF-36 subscales: role limitations due to physical problems, $d=-.034$, vitality (energy/fatigue), $d=-0.41$, and bodily pain, $d=0.22$. This represents a decrease in stress, depression, and unexpectedly, quality of life related to bodily pain. An increase in quality of life related to physical problems and energy/fatigue was observed.

Safety. Participants were asked during the exit interview whether there were any negative consequences as a result of participating in the study. Every participant affirmed that there were no negative consequences and no safety concerns were noted. Sleep efficiency $>95 \%$ was observed among one participant, P7, at all visits after Session 1. Elevated sleep efficiency $>95 \%$ is notable from a safety perspective, as it may increase the risk of motor vehicle accidents due to increased sleepiness. CBT-I protocol involves reminding participants with elevated sleep efficiency to nap when needed, for safety purposes. Although this participant did not drive for a living or operate machinery, this protocol was followed, and this participant was also reminded to continue increasing time in bed after study completion in order to reduce sleep efficiency into the target range of $85 \%$ to $90 \%$. The study therapist did not observe any adverse events among participants, and no safety concerns were reported during the study. All participants were advised to take a nap if they were sleepy and needed to drive. Similarly, participants were encouraged to put sleep restriction recommendations on hold if they became ill, and to resume adherence once they felt better. Several participants reported coming down with a cold or the flu during the study, and until they felt better, the therapist postponed their sessions as well as adherence to treatment recommendations and monitoring.

Acceptability. Participants were asked during the exit interview what they thought about the treatment, and if they would recommend it to people who had both HIV and insomnia. All 
participants reported that their sleep improved and that they would recommend the treatment to others. On the TEQ, a measure of treatment credibility, mean score increased from Session 1 ( $M$ $=5.8, S D=0.6)$ to the final session $(M=6.6, S D=0.4)$. A series of paired samples $t$-tests were run, applying bias-corrected bootstrapping to account for non-normally distributed data to examine whether this difference, and the difference of any specific TEQ items, was statistically significant. TEQ mean score was significantly higher posttreatment, $t(9)=-3.22, p=.011$, biascorrected 95\% CI [-1.20, -0.35]. Mean scores on two specific TEQ items also significantly increased from Session 1 to the final session. For the question "how confident are you that this treatment will be successful in eliminating your insomnia?", the mean score increased from 5.0 $(S D=0.9)$ to $6.2,(S D=0.7), t(9)=-3.15, p=.012$, bias-corrected $95 \%$ CI $[-1.90,-0.60]$. Similarly, the mean score for the question "how confident would you be in recommending this treatment to a friend?" increased from $5.7(S D=1.1)$ to $6.9(S D=0.3), t(9)=-3.34, p=.009$, bias-corrected $95 \% \mathrm{CI}[-1.90,-0.60]$. The maximum score for each item, and the mean TEQ score is 7 , which represents "very likely", therefore participants found the treatment credible after Session 1, and even more so upon completion of the treatment.

In terms of adherence to sleep prescriptions, participants spent an average of 33 minutes $(S D=29)$ longer in bed than was prescribed. This varied over the course of treatment, with the difference between prescribed and actual adherence decreasing over time from 41 minutes $(S D=$ 44) after Session 1 to 40 minutes $(S D=43), 28$ minutes $(S D=32)$, and 25 minutes $(S D=29)$, after Sessions 2 and 3, and the final session, respectively. Therapist-rated adherence to treatment recommendations, including CSD completion, was measured using a single item from the TCC, and mean adherence across all sessions was $64.8 \%(S D=15.7)$. Mean adherence per session was $96.0 \%,(S D=8.5), 50.3 \%,(S D=25.2), 53.7 \%,(S D=31.4)$, and $63.2 \%,(S D=23.2)$, for 
Sessions 1, 2, 3, and 4, respectively. Therapist-rated adherence for Session 1 is based solely on CSD completion, whereas for Sessions 2-4, it is based on adherence to treatment recommendations. Therefore, therapist-rated adherence to treatment recommendations increased from Session 2 to the final session.

The ability of participants to adhere to treatment recommendations, and to complete the treatment protocol provides evidence for treatment acceptability. In addition to the ten participants who completed the CBT-I protocol, one additional participant attended the first session, then became ill, then injured, and eventually stopped responding to requests to reschedule the second session. The decision was made to include this participant's baseline data in the full sample cross-sectional analyses. Given the pragmatic nature of the current study, the data were not used as intent-to-treat in the pilot study sample. Including this participant, however, in calculating attrition for acceptability purposes provides a conservative estimate of $9.1 \%$ attrition.

Feasibility. Feasibility was examined with regard to inclusion/exclusion criteria, recruitment and screening procedures, and CBT-I protocol. With regard to inclusion/exclusion criteria, the initial plan for the broader study was to exclude participants with suspected or diagnosed OSA; however, it was the most common reason for excluding participants during the telephone screen, with $14.1 \%(n=20)$ of participants screened being ineligible due to suspected or diagnosed OSA. Furthermore, 33.3\% $(n=16)$ of participants who completed the baseline assessment were identified as likely having OSA. Altogether, 25\% $(n=36)$ of all individuals initially screened were identified as likely having OSA. This frequency excludes the 36 individuals who were eligible after phone screen but who did not complete the baseline assessment, and who might have also met criteria for OSA using the DSISD. Given the high 
prevalence of OSA identified in this population during telephone screens and baseline assessments, and given the pragmatic nature of the study, it was decided not to exclude all participants with suspected OSA from the broader study who met remaining inclusion/exclusion criteria. It was decided, however, that for the CBT-I portion of the study only, individuals with suspected OSA would continue to be excluded. The rationale for this was that untreated OSA may impact treatment of insomnia, and the main goal of the CBT-I pilot study was to explore the impact of CBT-I on insomnia symptoms among PWH.

With regard to recruitment and screening procedures, some sources were more successful than others. During telephone screening, participants were asked where they heard about the study. In terms of recruitment sources for eligible participants who completed the baseline assessment, the largest recruitment source was through HIV/AIDS community organizations, with $41.7 \%(n=20)$ of participants recruited through this method. Organizations included the Toronto People with AIDS Foundation (PWA) and the PWA newsletter, the AIDS Committee of Toronto (ACT) including the ACT Positive Youth Outreach program (PYO), as well as unspecified community organizations. Online advertisements recruited $18.8 \%(n=9)$ participants, and included sites such as Facebook, Scruff, and Grindr. Another 16.7\% $(n=8)$ participants were recruited through posters placed in the community, and $8.3 \%(n=4)$ through posters placed in medical clinics specializing in HIV treatment. Finally, $14.6 \%(n=7)$ participants reported being recruited via word-of-mouth through friends who had heard about the study or participated themselves. Given the high proportion of participants recruited via HIV/AIDS community organizations, future studies recruiting PWH would benefit from aligning with HIV community organizations and incorporating print and online recruitment material. The 
PWA newsletter was a particularly successful recruitment strategy for this study, but was unfortunately not utilized until later in the recruitment process.

Recruitment for the CBT-I portion of the study was slow, with many eligible participants opting to participate in the broader study instead. The broader study involved just one single visit for a $\$ 50$ incentive whereas the CBT-I portion involved a total of six visits, each spaced two weeks apart, for a $\$ 60$ incentive and free insomnia treatment. Only $8.3 \%$ of the sample scored in the range indicative of severe insomnia on the ISI, so it is possible that insomnia was insufficiently severe, distressing, or impairing for eligible participants to commit to CBT-I. As mentioned below, CBT-I participants identified motivation as an important factor in treatment efficacy, and this appears true for the recruitment process as well. Recruitment of large numbers of PWH with insomnia severe enough to motivate them to commit to CBT-I may prove challenging.

With regard to the standard biweekly four-session format, only one participant (P5) received less than the standard four sessions, and this was collaboratively decided between the therapist and participant given the participant's early symptom remission. Participant P5's ISI score was in the range indicative of no insomnia after the first CBT-I session, and remained in this range over the following two sessions. Similarly, participant P5's sleep efficiency surpassed the target range of $85 \%$ to $90 \%$ after the first session, and returned to a value within the target range by posttreatment. Furthermore, participant P5 reported being satisfied with the progress after the initial three sessions. Given that the current study was a pragmatic pilot study, it was agreed that we would terminate therapy after three sessions instead of four. Two participants received more than the standard four sessions. Participant P4 requested, and received, an extra session in order to spend more time practicing cognitive restructuring strategies after time that 
was to be used for that purpose was spent troubleshooting behavioural strategies. Participant P9 took an extended vacation and was away for 84 days after Session two. Upon the participant's return, Session 3 was used as a reorientation in which Session 1 information was reviewed, and two sessions were added for a total of six overall — two before the vacation and a full four sessions upon reinitiating CBT-I. The pragmatic nature of this pilot study provided for some flexibility to the standard CBT-I protocol. Overall, the average number of days between first and last CBT-I session was $55.8(S D=15.7)$ excluding participant P9's 84-day absence between Sessions 2 and 3. For comparison, a four-session biweekly protocol administered precisely every two weeks would include 42 days between first and last session.

Administration of the CBT-I protocol took slightly longer to administer in the current study than it typically would. One reason for this is that several participants became ill over the course of the study, and were encouraged to put all treatment recommendations aside while they were ill, including sleep monitoring, and to only resume treatment recommendations and sleep monitoring a few days after they had recovered from their illness. Due to this recommendation, several participants took longer to complete the CBT-I protocol. Participants did not report any opportunistic infections over the course of the study, but reported illnesses such as the common cold or the flu.

\section{Thematic Analysis}

The exit interview had three specific objectives: 1) to elicit feedback about the treatment itself and the research study as a whole, including administrative aspects, in order to determine if and how CBT-I could be specially tailored to make it as acceptable and effective as possible for PWH; 2) to examine participants' knowledge about the CBT-I strategies presented during treatment and the extent to which participants found the strategies helpful; and 3) to gain a more 
complete picture of the impact of CBT-I on sleep-and HIV-related outcomes, as well as any other unintended outcomes that might pertain to the safety of participants. Although interview questions were structured to elicit information pertaining to these three objectives, the goal of thematic analysis was not to simply replicate these categories. Using the iterative process for conducting thematic analysis recommended by Braun and Clarke (2006), six overarching themes were identified: 1) Pre-Existing Needs; 2) Positive Impact; 3) Mechanisms of Change; 4) Challenges; 5) Suggested Modifications; and 6) Acceptability. Each overarching theme contained three to seven sub-themes. A thematic map is presented in Figure 37 to illustrate the association between overarching themes and sub-themes. Excerpts from exit interviews are presented to illustrate participant perceptions corresponding to each subtheme in the thematic analysis. Potentially identifying information has been removed, and participants are referred to here by a series of codes not used during data collection (i.e., P1 to P10). Although some participants were more forthcoming than others during exit interviews, comments from all participants are included in the thematic analysis.

Pre-existing needs. Participants reported pre-existing biopsychosocial needs, many of which are not directly addressed by standard CBT-I protocols. Sub-themes identified here were: 1) damaged body; 2) psychological distress; and 3) disengagement from society.

Damaged body. Some participants described their bodies as being physically damaged, whether due to living with HIV or other causes. Participants who mentioned this also noted its association with sleep problems.

Um, the napping [recommendation] was hard, because unfortunately, for me to even watch TV I have to lie on my side. So I had to change that too. I had to learn how to sit differently, because I've had a _ operation. So I could watch TV but not lie down. (P2) 


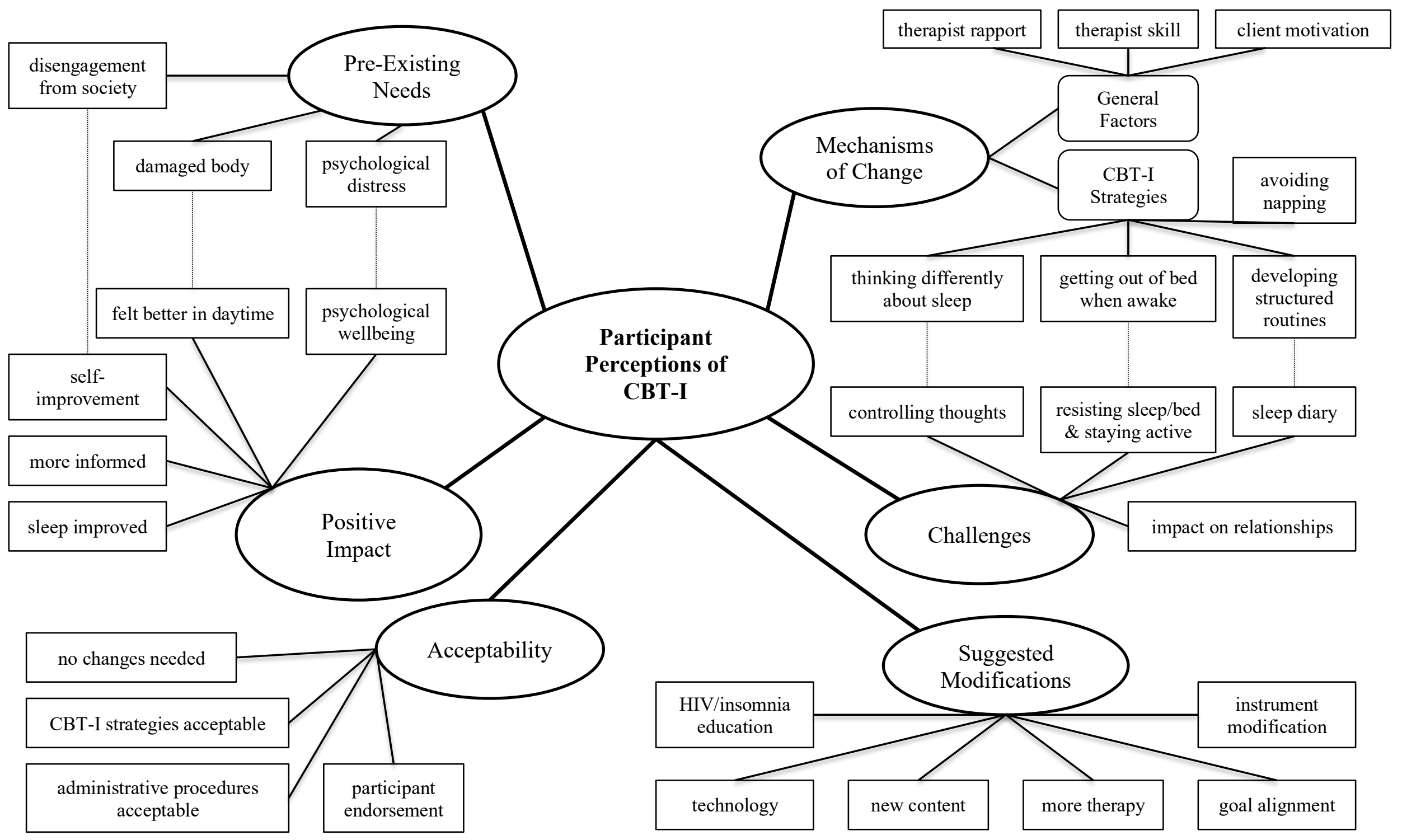

Figure 37. Thematic map of participant perceptions of cognitive behavioural therapy for insomnia (CBT-I). 
As a long-term survivor, you're living in a body that has been damaged by the experiment of finding the right pills. You're living as a person who takes a lot of pills. No one knows what this is going to do when you get older to your sleep. So it's all learning and finding out I guess. (P7)

Psychological distress. Participants expressed that within their community of PWH, grief and loss are widespread. At the same time, it was noted that CBT-I provided transferable strategies that could be used to manage grief and loss.

In our sector [the HIV sector] for example, there's a lot of loss and grieving...you know, you lose [people] one way or another. More often than you might wish. And those coping strategies also work for grief for example, or other kinds of loss. (P5)

Disengagement from society. Participants expressed a sense of disengagement from society across a number of domains: financial, occupational, and social. They described poverty limiting their ability to fully engage in treatment recommendations.

Or maybe a discussion about money: At one point we talked about was keeping your day full. Right? Well if you're HIV and on ODSP [Ontario Disability Support Program] and don't have a lot of cash? So a discussion about activities that don't cost any money would be a good thing to throw in there, like "did you know you can do this?" to help keep your day full. (P4)

In my day when we got diagnosed, the first two things they told you to do (and I was diagnosed in ): stop work and do your will. So the biggest thing I miss, is going to work. (P7)

My impression on reading [the homework exercises and handouts] sort of at first blush is that they're geared for a population that's employed and so therefore they have 
an issue where they want to sleep well so their performance can be better in their employment activity...'Cause it took a hurdle for me to shift my mind from “why do I need to do this? I can just nap all afternoon anyways," not that all HIV people are on ODSP and not working, but I can imagine that that's a pretty big component as to why do we care? Why bother? (P4)

Positive impact. Participants reported that CBT-I had a positive impact on sleep as well as a number of other areas that were not specifically targeted in treatment. Sub-themes identified here were: 1) sleep improved; 2) felt better in daytime; 3) self-improvement; 4) more informed; and 5) psychological well-being.

Sleep improved. All participants reported improved sleep after receiving CBT-I. They referenced improvements to sleep quality, consistency, duration, and number of wake-ups. While some described making progress with room for more improvement, others expressed surprise that their sleep problem had resolved so completely and quickly.

I'm sleeping shorter hours and that the sleep that I'm getting seems to be better...the quality seems to be a little deeper because he got me on a regimented time, which for me was necessary. (P1)

I completely—how can I say? When you —oh my god I forgot the word. I recovered my sleep, like $100 \%$...I would say I didn't even imagine that that would be so fast. Like the first two weeks I already recovered...I would say it really works, it really helps. It should be a first option, instead of trying whatever else...medication or I don't know what else. (P3)

I'm sleeping better, and by better I mean I'm sleeping longer and more consistently, so I've learned to have a sweet spot of time that runs, and within that I'm 
waking up less, like instead of six or seven times a night, you know, three or four and consistently getting around six hours of sleep, which is an improvement from four and a half to five, and feeling more rested, and feeling more confident about my ability to manage my sleep. (P4)

I'm still having some difficulty but it's more so with my behaviour than it is my sleep...like not really having good bedroom hygiene, and like, things that I'm very aware of, but might not be sticking to. (P9)

Felt better in daytime. Participants reported feeling better in the daytime, highlighting improvements in energy and alertness. They also noted less daytime interference from insomnia and fatigue.

I do feel like I have a lot more energy during the day than when I had started. (P9) It [sleep] kind of permeated throughout the rest of my day, I would try to avoid doing stuff because I thought it would impact my sleep later. Or I didn't feel like doing other things, because I was groggy and tired. But now, in the last few weeks, that's pretty much gone away...I don't feel as groggy and tired throughout the day. I feel like I have more energy and it's just like, it's not really a concern as much. (P8)

Self-improvement. Participants reported greater confidence, control, productivity, selfesteem, and sense of self-empowerment after receiving CBT-I. In many cases, this selfimprovement was attributed to CBT-I strategies that were perceived to be useful in other areas aside from sleep. For example, participants reported using CBT-I strategies to improve their experiences at work, for becoming more active and having a more rewarding life, and for dealing with grief and loss. 
I feel it's given me the confidence that I can be more in control of situations that I thought were perhaps less in my control. You know the fact that if I can use, a relaxation technique, or a focus technique to help me sleep, it also works in other applications, a little differently, but same general gist... and If I need to hone in and focus on something I can just call upon that same sort of set of skills at least, and use it in those kinds of things, which I've actually found useful in my work. (P5)

Yeah, and also I feel more confident about everything I learned from this treatment and even in the future if I have any problems with insomnia, I feel that I learned ... know how to deal. I have so many tools to deal with that. So, I feel very confident...It helped my routine especially, like my development at work. My routine in general, even not at work, like weekends and my day life changed for better, like my quality of life changed. (P3)

Also, it gave me something to do. It made me feel—sometimes I go through the day and talk to cashiers and go to medical appointments - for me this felt like a job and it made me feel good doing something over a longer period of time. (P7)

More informed. Participants reported that CBT-I provided information about sleep that corrected misinformation and informed strategies for improving sleep. They specifically mentioned the sleep diary as a source of valuable information, as it is based on their individual sleep data. They noted how this information would help them recover from insomnia in the future in the event of relapse. Participants expressed appreciation for this new knowledge. It helped me to demystify things in my mind, like before I started I always was worried about how many hours I had to sleep, and why I don't sleep continuously. Like I had so 
many questions in my mind that worried me a lot. And now I know that this is not a big deal. (P3)

I would think it was the learning about the cycles of sleep. I guess, as an example, I had always assumed that, because I remember it most, that falling asleep and falling back asleep and then that last hour or so where you have the amazing dreams that was the good part to get to but I've learned that that, in fact is not the case and the good part is the very beginning so you set your room up and your behaviour up so that you maximize that early part of the sleep cycle. So just brand new knowledge was really good. There's a science behind it I guess. (P4)

It's made me more aware of: 1) What contributes to insomnia, but how to, through monitoring that and through understanding to what affects it, how to improve, as well as the techniques of how to deal with things that affect my sleep and affect my ability to get to sleep or stay asleep. (P9)

Psychological well-being. Participants reported reductions in anxiety, depression, and frustration, and attributed this to the treatment, and in one case, the thought record specifically. But I-first I thought maybe it's because I switched medication, that my sleep improved, but then I realized that it wasn't, because I-my anxiety was gone. And this is not-I don't think there is something to do with the medication, it's about the treatment here. And my sleeplessness was maybe $100 \%$ about anxiety... So, yeah that made me feel like my anxiety [whoosh sound]. (P3)

I'm just generally less anxious. Because sleep was such a big problem for me before, it kind of permeated throughout the rest of my day. I would try to avoid doing stuff because I thought it would impact my sleep later, or I didn't feel like doing other 
things, because I was groggy and tired. But now, in the last few weeks, that's pretty much gone away... yeah, less anxious, I don't think about it as much. (P8)

I'm not getting as frustrated with myself. But not sleeping, that continued all day. If you haven't slept all night, you don't want to go to bed because, you know, something is going to happen, you're not going to get to sleep. (P2)

Mechanisms of change. Participants reported which factors they perceived to be responsible for sleep improvement. These included specific cognitive and behavioural CBT-I strategies as well as more general factors which overlap with a set of common factors in psychotherapy known to impact treatment outcome (Wampold, 2015). These common factors include goal collaboration, empathy, alliance, positive regard, genuineness, cultural adaptation, the therapist her/himself, and treatment expectations (Wampold). Specific CBT-I strategies reported as helpful by participants included: 1) thinking differently about sleep; 2) getting out of bed when awake; 3) avoiding napping; and 4) developing structured routines. General factors participants reported as helpful were: 5) client motivation; 6) therapist rapport; and 7) therapist skill. While client motivation is external to CBT-I and the common factors, it was identified as essential to treatment success, therefore the coders decided to group it under the "mechanisms of change" theme.

Thinking differently about sleep. Participants reported that thinking differently about their sleep was helpful. This sub-theme included references to specific cognitive restructuring strategies introduced during therapy such as the thought record and constructive worry exercise, with participants describing their own ways of challenging thoughts, such as using mental images rather than words to consider alternate outcomes, and using smartphone applications to engage in constructive worrying. Participants also noted changes in perspective about occasional 
sleep disturbance, and how these were important factors in reducing anxiety and improving sleep. These changes in perspective reflect increased acceptance about occasional sleep disturbance as well as an understanding that worrying about sleep does more harm than good.

Um, don't worry about it so much. Worrying about insomnia only feeds the insomnia. So, get over it [laughter]. (P5)

Try not to- if I don't sleep, for example, if it's difficult for me to fall asleep one day and the next day, I know that I'll probably have a better sleep - not in terms of time, but in terms of deepness. So, don't worry about catching up in terms of time. Like okay if I sleep seven hours a day, the next day—and this night I slept like four hours or three, I don't necessarily need to sleep ten hours the next day. Because I'm probably going to be tired enough to have a deep sleep. So yeah, a few things that usually bother me a lot, now I know that...I don't worry about—don't even think about. (P3)

And well hopefully the insomnia wouldn't return because I would feel the oncoming of the thought patterns and start changing them then. It's probably just for me it works-I'm very visual, so when we did the thought thing I had a real difficulty, first of all, explaining it in words, and then changing it in words. So now what I did for myself is I look at it as a little movie in my head, so whatever the negative thought was, in a movie, I rewind the movie, and change the way the movie goes, and that seems to work for me. (P2)

Getting out of bed when awake. Participants identified one of the important stimulus control guidelines of getting out of bed when unable to sleep as helpful. Also included in this sub-theme are references to not using the bed for anything except sleep or sexual activity, another stimulus control guideline, and limiting time in bed to match the amount of sleep 
produced, a sleep restriction guideline. Participants specifically referenced the importance of associating the bed/bedroom with sleep and not with other wakeful or arousing activities.

Get out of bed if you feel yourself alert and awake in bed. That strategy works pretty well for me. I usually go out and go watch something, a film or whatever I already watched, so I don't have this expectation about what's the end? So I know, and then I feel pretty relaxed. (P3)

I was spending a lot of time in my bed just like hanging out in my room as a hangout spot and I have definitely made that a better part of how I spend time at home. While I still do hang out in bed sometimes, I have like a little area set up in my room so I can hang out outside of bed... Well I think those were two areas that I was kind of overlooking as being contributors to my sleep problems, and being more cognizant of that played a role in my ability to sleep was helpful. (P9)

I think the first one, "match the time you spend in bed trying to sleep with the amount of sleep your body's producing," was probably the most helpful, because I tended to lie awake, especially if I had woken up during the night. And so I guess over time my mind wasn't associating bed with sleep, because I was lying awake. So shortening the window of time I spent in bed was helpful...Yeah, I think the common theme between all three of them is just not lying in bed doing nothing and allowing your mind to think about other things than sleep, and really trying to limit the amount of time I spend in bed to just sleep time, and then forcing myself to get out of bed if that's not going to happen. (P8) Avoiding napping. Participants identified another sleep restriction strategy, avoiding napping, as helpful at improving their sleep, with specific reference to its impact on sleep drive 
and the importance of building sleep drive throughout the day. At the same time, many also identified this strategy as challenging (see next section).

Avoid napping, because I was a big napper before I came in and he admonished me about that. So I cut down my napping and I felt, therefore, I was tireder when I went to sleep. (P1)

Except for one time when I was sick, well actually the pain thing, avoiding napping. It just disrupted — and I actually sort of watched when I did do it. Yes, sure enough, I felt better for the moment, but the next day was worse because I hadn't slept soundly. (P5)

Developing structured routines. Participants reported that having consistent, structured routines helped to improve sleep. This included using the CSD, adhering to the sleep prescription, and practicing self-discipline. The noted that adhering to sleep prescriptions was not always easy, and required self-discipline, but that it was effective.

In the first two weeks I hated doing the diary. I felt it was too many questions to answer the next day. But the moment I saw the results when you put it together, he was able to identify how many hours of sleep I needed and then gave me, he was able to show me, what we were going to try next, and every time I did what he said, I saw what he wanted to accomplish. (P7)

The most useful? To go back to a good sleep routine. And to help me, especially on weekends that I used to try to catch up sleeping, so I kind of lost Sundays trying to sleep... so, that night I felt an improvement in my routine and my weekends, and at work it helped me a lot to be more focused and less tired. (P3) 
Because I actually had a week during this process where I had the flu. So I was in bed, off and on, 24 hours for a week, and I was like, oh crap, I'm not going to be able to get back into the sequence again, but knowing that, if I go to bed at 2, and get up at 7:30, I'm going to have a refreshed sleep. Knowing that in my head actually helped me get back into the pattern really quickly, really quickly. And that was like, oh ok, this does work. (P2)

Client motivation. In response to a question about what might prevent other people living with HIV from benefitting from CBT-I, several participants expressed a belief that treatment success depends on high motivation and effort. As mentioned above, motivation is not a treatment component, and therefore, not technically a mechanism of change, but was included in this category as participants stressed its importance in successful implementation of CBT-I. I think you have to want to get sleep. Like I don't know, I'm in other programs where people don't want it-you're not going to be convincing them of anything. (P1)

If you're not willing to bend; if you're not willing to modify behaviour as needed to do what you need to do for this. Some people say "I want help," but they really don't want to change anything. They just want something to magically drop from the sky and change them, or change their circumstance. And, like, in pretty much anything else, that just isn't realistic. It doesn't happen, for anything. So you have to be willing to try each step, each — the little take-home tools. You have to be willing to listen and be-what's the word - intentional, about what you do. And actually really try to do what is being talked about. The strategies we looked at earlier-you have to be willing to try those. Again, some might work better than others, some might be less applicable, but if you're 
not willing to do any of them, you're just wasting your time and money. And the counselor, of whatever nature, their time and money too. (P5)

Therapist rapport. Participants mentioned appreciation for their relationship with the therapist at various points during the interview. Specific features of the client-therapist relationship mentioned included being approachable, understanding, interested, friendly, patient, and a good listener. Although not all participants explicitly associated this relationship with treatment outcome, there is evidence that therapist alliance is one of the common factors associated with treatment outcome (Wampold, 2015).

Very easy to speak with, almost immediately. He's engaging in that sort of nonthreatening sense that's useful in a counselor or person where counseling is involved. Yeah, really excellent and approachable. (P5)

And he understood what I was talking about, even with the age difference. You don't see that. I'm _, he's got to be 30 — don't tell me his age—and you know, it surprised me how attuned he was to what I was going through. (P7)

He was great, I felt that he was interested in me, that I wasn't just another number in his list... He did—well I felt that he did—he was curtailing [participant likely meant "tailoring"] it for me. Like I felt that I was an individual. Yes the thought patterns, the techniques are all universal, no matter what you come into the therapy class to do, those are universal. But I felt that he would sort of gear it towards me personally, so if somebody else was sitting here coming up with stuff, it would be a little bit different if I was sitting in on their session, so that makes you feel like it's worth it. (P2)

Therapist skill. Participants expressed confidence in the therapist, in terms of knowledge and skill. They reported benefitting from understanding the rationale behind treatment 
recommendations. One participant also expressed an appreciation for corrective feedback received from the therapist. Participants commented on therapist traits they found helpful. Everything he did he explained why. He, like, as I said with the CBT, he talked me through it every step of the way. Nothing that he said didn't have a reason behind it, and also I felt very comfortable telling him anything, and also when he noticed things like my napping and also how much coffee I'd drink he wasn't hesitant to tell me to stop it. (P1)

He was knowledgeable about what he was doing, that's for sure... and he was always here when I was here, he was never late, you know, he's, was always on time and stuff. (P2)

Challenges. Participants identified challenging aspects of CBT-I and provided rationales for some of these challenges. Sub-themes identified here include: 1) resisting sleep/bed and staying active; 2) impact on relationships; 3) sleep diary; and 4) controlling thoughts.

Resisting sleep/bed and staying active. Participants reported that resisting opportunities to get more sleep was very difficult, as was staying active during the day. Challenging recommendations included getting out of bed if unable to sleep, adhering to a consistent wake time, and avoiding napping. Some participants identified that not having daily routines or reasons to be up at a certain hour made it more difficult to adhere to recommendations. Others attributed adherence difficulty to fatigue and medication side effects, noting that low energy and medication side effects such as dizziness, difficulty concentrating, and blurry vision made it very difficult to be active during the daytime and increased the likelihood of lingering in bed and napping. 
In terms of time in bed and stuff, when you don't have an obligation every morning when you have to get up, that makes it really hard to do it. I hope when I get a part time job it'll fill the day up better so it will be easier. (P4)

I wouldn't be able to sleep some nights and I'd finally get sleep and I'd be like oh shit, sorry pardon my language, but I have to get up now. So trying to stick to those hours was very challenging. And sometimes I could do it and sometimes I couldn't. I could do it more often than I used to but not as much as I think he would've liked. (P1)

Sometimes I find it very difficult to get out of bed when I wasn't sleeping. I kind of pushed myself at times, but there were other times that I didn't. Wishful thinking that I was going to get back to sleep. (P10)

So, napping--I did find myself needing a nap sometimes. Just having zero energy or, like, will to stay awake until—and this is usually in the evening, when I could nap or I could do an activity, but, um. Yeah. I found that, like, I would need a nap before going to the gym, as an example, and then I was kind of like, kind of trying to avoid napping. So that was one that I, I found myself, even though I understand the logic behind it, I found myself often times needing a nap, and it's just like short naps, and then sometimes extended to longer naps. (P9)

Impact on relationships. Participants reported that some treatment recommendations were challenging because of the impact they were perceived to have on relationships with friends, partners, or pets. Implement a wind-down period before bed. That was a little bit difficult for me, especially on weekends, because I feel very bored on weekdays and on weekends I like to break the rules and don't think about going to bed soon. Like I just want to spend time 
with friends. So that was a little difficult for me, but more on weekends, to implement a wind-down. Especially on Saturday [laughter]. (P3)

For me one of the challenges was having a social life on the weekends, but, and that was always a kind of a problem that I would stay up and just be up all night on the weekends and then sleep all day and then during the beginning of the week I would feel the effects of that, and I didn't... while I was kind of addressing it during the treatment, there were a few times where I made those noticeable slips around weekend sleep that I could definitely feel how it affected me during the beginning of the week. (P9)

Umm, being a non-single person, therefore, if I'm trying to follow a certain behavior and what I do before I go to bed, but my partner is in a different space, that can really challenge the process, and having to be able to assert myself to say, you know, it's really fine if you go to bed earlier, or later, but this is how I have to do it. And it used to be that I would just say, “ok, you're going to bed, I'll go to bed too", or it's like, "oh I'm tired, are you coming?" I've managed to, with some difficulty, sort of separate that out and say, no, this is what I need for me. (P5)

Sleep diary. Several participants identified that the CSD was challenging to complete, but most noted that this became easier over time. Reasons for finding it difficult included forgetting to complete it, finding it burdensome, having difficulty estimating sleep/wake time, striving to be precise.

In the first two weeks I hated doing the diary. I felt it was too many questions to answer the next day. But the moment I saw the results when you put it together, he was able to identify how many hours of sleep I needed and then gave me, he was able to show me, 
what we were going to try next, and every time I did what he said, I saw what he wanted to accomplish. (P7)

The sleep log that I had to keep, again I understand why it needs to happen, but it's tough to do it consistently, and I know that he wasn't looking for exactly to the minute of how long you were sleeping and what time you got out of bed and what time you woke up and stuff, but there were some nights where I was like, "I have no idea how long I've been awake, I don't know how you'll fix that." But then trying to piece it together the next morning was a little tough some nights. Most night were fine though.

It's a little painful when I started, because I wasn't sure about if I was so precise, if I would be able to be precise in terms of how many minutes I woke up during the night. But then after two weeks I noticed that I could be more precise than when I started. (P3) Controlling thoughts. Some participants acknowledged that examining their thoughts about sleep was challenging, if not counterproductive. Interestingly, one participant (P2) who identified this strategy as being counterproductive also reported finding it helpful when applied to other areas aside from sleep (see above).

Examining your thoughts and beliefs about sleep and your ability to function while during the day. That was hard for me, because I over examine things, I overthink a lot. So it's hard for me to see things from another perspective. Like to examine myself, like the thoughts and beliefs. That was the most difficult for me, but then Tyler gave me a spreadsheet that he gave to me that I filled with some - there are columns that you fill, like what is your problem, like what should you-why are you worrying about it, or what 
do you think in a positive way about it, what do you think should happen if your thoughts would be true or what happened? So this kind of exercise for me helped. (P3)

I don't know about the examining my thoughts and stuff like that. Um, you don't necessarily, I don't really have a lot of control over that at times. (P10)

The counting your thoughts one, for me was just, that would just make me go even more haywire; examining the thoughts and beliefs about sleep, and my ability to function well during the day. I just didn't associate them, like even if I did, it made it worse. (P2)

Suggested modifications. Participants provided helpful suggestions for tailoring CBT-I for PWH. Sub-themes identified here include:1) HIV/insomnia education; 2) technology; 3) more therapy; 4) goal alignment; 5) new content; and 6) instrument modification.

HIV/insomnia education. Some participants expressed a desire to learn more about the connection between HIV and insomnia, as well as the role of HIV medications in insomnia. Others did not think that insomnia was associated with HIV, at least for them personally. One participant expressed surprise that other health care providers did not acknowledge the association between HIV and insomnia and were not forthcoming with information about this association.

Because if the purpose of the study is because there's a higher rate of insomnia among people with HIV, right? So maybe more, kind of insights as to why. Like, maybe it's understanding how - I mean that's kind of outside of the treatment, but - it's, to me, it's something that is interesting, like why do people living with HIV have a higher risk of insomnia? Is it because they're more likely be dealing with mental health issues, or is it because, I don't know, they may be more likely be using drugs? I'm not sure. Like, who 
knows. Some context around how people living with HIV are different, if different, from the general population. (P9)

Um just more knowledge as to what you guys think is the reason behind the connection between HIV and insomnia because I'm not leaving with any idea, any more of an idea now than I did when I walked in the door, because that kind of troubles me. Because Tyler and I talked about it in the sense like, is the insomnia because of the HIV or it is insomnia because of the HIV medication. And we didn't, I didn't really get an answer to that...I wanted to know more about the details of, and also, I don't know if you would know this, but about the medications themselves. (P1)

I'm not convinced, myself, that by changing my sleep patterns, have anything to do with my HIV. I don't see a lot of correlation there. And maybe I'm missing something, but for myself. (P10)

Technology. Participants requested an electronic or online version of the sleep diary and other tools to make them more convenient, noting that they sometimes wanted to complete exercises when they were away from home and had access to their smartphone but not their homework handouts, and that they might forget what they wanted to write if not done immediately. They also requested to have the questionnaires available online to complete at their convenience, but at the same time acknowledged that from a research perspective, it may be problematic to have participants complete questionnaires in uncontrolled environments. One participant expressed a desire to use text messaging to coordinate appointments, noting that emails are missed when they end up in the junk folder.

Maybe instead of adding this data on paper, maybe an app or something that I could do while I was on the TTC...Yeah, on an app or it doesn't necessarily need to be on an app. 
Maybe on a website that I go and add the data and have access later. A tool that I can keep updating and comparing, like even later in the future, if I have problems with insomnia again. (P3)

Yeah if that sleep log could be filled out in like an excel spreadsheet instead of a hard copy, because there was a couple times where I was halfway to work, because it was a session day, and I realized I forgot it at home, so I had to go back. If I was able to keep it electronically, and maybe there'd be away so Tyler didn't have to manually input it into whatever system he inputted it to. (P8)

I've thought about would that be easier if you could just log in at home and do it, but then there's that whole problem of, is it really the person doing it? In a study you really have to be tight with your material. You have to know that you're getting equivalence, getting the same info from the same person at a different time, for example. So I'm not sure there actually is a way to simplify that, as much as it would be nice if you could just sit at home and do it in advance, it's like, oh yeah it's done, see you can check. (P5)

More therapy. Many participants requested more therapy, including more sessions to fine-tune the sleep prescription and troubleshoot any lingering problems, longer sessions to spend more time practicing strategies (specifically cognitive restructuring), more practice with specific strategies in-session, follow-up contact with the therapist a few weeks after therapy ends for general check-in on continued progress and troubleshooting, and making CBT-I more accessible to the community by implementing it in HIV/AIDS community organizations.

I mean what I would change is the length of it. Like I would change the pattern. I think is what four weeks now? I mean, to me, I didn't get enough time-I haven't had enough 
time practicing it. I would have needed - I felt I needed another two weeks to just do it...just actually doing the practice, of going to bed at and getting up at . And seeing if there was anything there that wasn't working. (P2)

I think one of the sessions- so the analyzing the negative thoughts section, it was tough to explain it and then go through an example in the detail needed within just the hour we went over, so maybe not necessarily being confined to an hour for every session if that session might need more than an hour in order to grasp the strategy and the homework. (P8)

At some point it could even be incorporated into an ASO I think...mm-hmm, because a lot of agencies provide all kinds of counseling type, or referral services, or even in-house. Why not build this sort of element into some of that counseling referral that they do? (P5)

Goal alignment. Although some participants reported that CBT-I seemed to be tailored specifically for them (see mechanisms of change, therapist rapport subthemes above), others expressed that treatment recommendations, including the sleep prescription, did not align with their personal goals. One participant (P1) expressed a belief that the sleep prescription was assigned without any choice being offered, which is not how sleep prescriptions are determined in CBT-I, however, it is noteworthy that this was the impression he received. One participant noted that this misalignment resulted in poor treatment adherence. Participants also recommended spending more time getting to know them before starting therapy.

The initial interview should be longer and, so he can get a better understanding of me...I don't remember if we had an initial, we did it on the phone, but there should be some sort 
of initial appointment just to understand each other so I don't go through that in my therapy sessions. (P7)

I mean, sometimes with the recommendations that were given to me, I think that they weren't totally in line with what my goals were, and again it goes to the "me" element of me not really adhering to some of the recommendations that were made. (P9)

I had a problem with the timeframe that he gave me, was midnight to seven am and I almost, and this is just between you and me, but I'm just going to say this, I wasn't really given a choice as to when I was going to start, when I was going to finish. It was more sort of said to me, like, let's do this and this. I didn't protest, I didn't say anything, but I almost felt like I—-this is my fault—I should've told him that maybe it should've been adjusted to eleven to six. Like, the total hours was totally fine, but the timing of it, and it's my fault because I didn't speak up and it could've changed as to-maybe I should have more say or something. (P1)

New content. Participants suggested having a more explicit outline of what to expect in the coming sessions, such as a "roadmap" for upcoming sessions (P7). One participant (P8) reported having difficulty generating examples of situations to discuss on the spot, and expressed a preference for receiving advance notification about what types of situations to think about in preparation for the next session. Other participants suggested adding content to address substance use and assertiveness, two issues they believed to be relevant to PWH in general, and also for overcoming insomnia.

Um, there's a lot of people with HIV that use recreational drugs. I don't know how-I didn't see that there was much of a focus on that, and how it might affect some people with their sleep and stuff. (P10) 
It's my belief that a lot of gay people with HIV are doing a lot of other drugs as well—at least in they do, or a lot of people do, like it's not $1 \%$, it's more

like 20, so that would be a large part of the population. And then getting them to say "it's ok to be doing the study as long as you're not overdoing it, but we want you to see the relationship of what you did Friday night and how that screwed up your whole week, because we're trying to get you to see how to improve the quality of your life and you can improve adherence to the medications." (P4)

But maybe one of the things that can come up in the midst of this is that if you think the meds are making it worse, that you have the power to go to your doctor and talk about changing your med regimen, or at least checking out the possibilities of it. Cause again, that part of taking charge includes saying, yeah, this is my body, I get to say, or I get a say in what goes into it, and if I'm feeling this effect, I think being able to claim that and say—have the wherewithal to go to your doctor, and say—look this is my need, this is how it's affecting me, we need to look at a change. And that's a difficult thing, but I think with some of the other esteem issues, connecting the — having enough self-esteem and self confidence that one can go to the doctor and assert oneself to make that change. (P5)

Instrument modification. Participants identified ways to make the CSD more useful for $\mathrm{PWH}$, by adding rows to track substance use and timing of HIV medications. They also identified problems with some of the questionnaire items used in the assessments.

Oh the sleep diary itself I did talk about caffeine intake and I'd maybe have another category like "other" like "caffeine, alcohol, and what else are you doing?" because it's my belief that a lot of gay people with HIV are doing a lot of other drugs as well...Oh 
that was another thing, I started putting in my medication times just partly for my purposes, 'cause okay, ya, 'cause what I became aware of after the first or second session was organizing your day-to-day life, and that's such an important part of it, is going to impact on your sleeping too. So if you take your meds at midnight and you go to sleep, how does that disrupt it more than if you have them at seven. 'Cause I could see that as something that could be very disruptive for the sleep pattern. So if somebody is doing them, which we all are, that that might be something worth tracking. (P4)

Oh, the one thing, specifically to the sleep log, this is getting very granular, but the sleep $\log$ was self-explanatory except the one issue I came up with was it asked when you got into bed and when you fell asleep, there were a few times where I got into bed and after ten to fifteen minutes my brain wasn't going to let me sleep, so I got out of bed, and then I did whatever to relax myself and maybe thirty minutes later I got back into bed. So there's no real way to capture that, because if I put in the time that I got into bed versus the time I got to sleep, then it would look like I laid there for an hour, when actually I got up. So I didn't really know how to capture that. (P8)

Acceptability. Although participants identified challenging aspects to CBT-I, and suggested ways that it could be tailored for PWH (see above), there was a consensus that this was an acceptable treatment. Sub-themes identified under treatment acceptability include: 1) no changes needed; 2) CBT-I strategies acceptable; 3) administrative procedures acceptable; and 5) participant endorsement.

No changes needed. Although participants suggested changes to CBT-I to make it more helpful for PWH, they also indicated that changes were not necessary, as the treatment was 
effective as-is, and the specific strategies introduced were already generalizable. This pertained to CBT-I itself, the therapist, as well as administrative procedures.

Nothing. I think every two weeks was good, an hour was good. (P1)

Oh my god, it was so successful for me. I can't even imagine how it should change, really. (P3)

I think that, especially with the...the more self-reflective/writing down your thoughts or concerns or to-do lists, it's really hard to tailor that for someone with HIV because it's just general, right? (P9)

CBT-I strategies acceptable. Participants reported being able to overcome many of the initial challenges, and that the strategies were feasible to practice and use. Several participants mentioned that while the sleep diary was initially challenging, it not only became easier over time, but became a very helpful part of therapy. One participant mentioned appreciating that strategies did not include medication. Others mentioned that examples on homework handouts were useful. Participants had mixed feedback regarding the ease and simplicity of CBT-I, but overwhelmingly agreed that regardless of whether it was challenging or a simple, quick fix, it was ultimately acceptable.

I like that it's on paper and you can fill stuff in, and the dairy takes a bit of getting used to because you're thinking about it so much that it keeps you awake, but once you get past that, it's putting your actions to pen and paper and then seeing the next day either the good result or bad result is a very, very helpful process. (P4)

They're [homework examples] all essential, and I was glad that he went over the sample when we were here, and there was a sample for me to take back with me home, so 
it was still there to look at, to say, "Ok, this is what he said and this is what he meant, this is what we can do." (P2)

I like how it focused my attention on behaviours I can control, and kind of demystified the whole insomnia thing, because after a while you start to believe it's me, it's my problem, it's something wrong with me. So yeah, I think the behaviour approach is more helpful than anything that I've experienced before. (P8)

I would say that it was a practical, useful, pretty straight-forward tool. (P5)

That it was something you could rectify with not all that much effort. You know, no pain. (P10)

Administrative procedures acceptable. Participants reported that administrative aspects of the study, including environment, amenities, and scheduling were acceptable. They also noted appreciating flexibility in scheduling, appointment reminders, being offered refreshments, and setting agendas.

The coffee was great, um [laughter]. No, the room was perfect actually, that, this was good for this too. It's quiet, um, there's nobody here, the lighting's subdued, it's all—the environment was good for it...And it wasn't a big deal filling that form every week either cause every time you go to the hospital you fill those stupid things in these days [laughter]. (P2)

I'd say overall, they were pretty well structured. The timing of the appointments were pretty flexible, so no issues there. (P8)

One issue that might come up, well you're on a roll and talking about something, and most therapists would say "ok I'll see you next week"- "OH! We're just at the good part," but then he was - he went overtime, I always felt when I left that what we were 
talking about was talked out fully, and that's something very few therapists if they have the time and are able to do that it's a plus. (P4)

Participant endorsement. All participants reported that they would recommend CBT-I to a friend living with HIV and insomnia. Furthermore, many reported that they had already told friends about it.

Yes, as a matter of fact, I've talked about this study with lots of friends who have sleep patterns...I've been sharing this with a number of people. (P10)

Definitely. Because I'm telling them all now! Over lunch or...and...I

definitely...this is an area I never of thought there's an expertise in. (P7)

Um, if I thought that person believed in this type of techniques and treatment, yes

I would. Totally. And I have done. (P2)

\section{Summary of Results from CBT-I Pilot Study}

The first hypothesis, that participants would experience a significant reduction in insomnia symptom severity, was supported. From pre- to posttreatment, mean ISI score reduced from a range indicative of moderate insomnia severity to one indicative of no insomnia, representing a large effect size. ISI score for all but one participant reduced by $2 S D$ by posttreatment, with this reduction occurring after just one session for four participants. Clinically significant improvement was observed for all but one participant, and half of participants met criteria for insomnia remission at posttreatment. Reduction in insomnia severity was associated with reduction in dysfunctional beliefs about sleep (worry/helplessness subscale), sleep effort, depression, anxiety, and stress, and an increase in quality of life (fatigue/energy subscale).

The second hypothesis, that participants would experience an increase in sleep efficiency to within a target range of $85 \%$ to $90 \%$, was partially supported. From pre- to posttreatment, 
mean sleep efficiency increased from $65 \%$ to $89 \%$, representing a large effect size. Sleep efficiency increased by at least $2 S D$ for all participants, with this increase occurring after just one session for seven participants. Sleep efficiency ended in the target range for just two of the ten participants, however, and it surpassed the target range for five participants, and was below this range for the final three. Sleep efficiency remained in the range indicative of insomnia for just one participant at posttreatment. Increase in sleep efficiency was associated with decrease in dysfunctional beliefs about sleep (medication subscale).

The third hypothesis, that participants would experience a reduction in total wake time, was supported. From pre- to posttreatment, mean total wake time reduced from 183.1 minutes to 44 minutes, representing a large effect size. For all but one participant, total wake time reduced by over $2 S D$, with this reduction occurring after just one session for seven participants.

The fourth hypothesis, that participants would experience an increase in HIV viral load, was not tested, given limited data available for analysis. There was no variability in the data, with all participants with blood test results available reporting undetectable viral load at baseline and posttreatment.

The fifth hypothesis, that participants would experience an increase in CD4 count, was not supported. Although a small increase in CD4 count was observed, representing a small effect size, this increase was neither clinically nor statistically significant. All participants reported CD4 count within the normal range for adults with healthy immune functioning at baseline and posttreatment. Increase in CD4 count was associated with increase in total sleep time and quality of life (social functioning subscale).

The sixth and final hypothesis, that participants would experience improved cART adherence, was partially supported. On a single-item self-report measure of adherence, the 
number of participants reporting their adherence as "excellent" increased from $70 \%$ at baseline to $100 \%$ at posttreatment. On a composite dichotomous measure of adherence based on several self-report responses, the proportion of participants classified as adherent decreased from $40 \%$ at baseline to $30 \%$ at posttreatment, however, missing data precluded useful interpretation of this result.

Large effect sizes were observed for reduction in time in bed, number of awakenings, wakefulness after sleep onset, terminal wakefulness after sleep onset, sleep effort, dysfunctional beliefs about sleep (full scale as well as worry/helplessness subscale), and fatigue. A large effect size was observed for increase in self-efficacy for sleep. Medium effect sizes were observed for reduction in sleep onset latency, daytime napping, dysfunctional beliefs about sleep (perceived consequences of insomnia and sleep expectations subscales), pre-sleep cognitive arousal, and number of bothersome HIV-related symptoms. A medium effect size was observed for increase in total sleep time. Small effect sizes were observed for decrease in depression, stress, dysfunctional beliefs about sleep (medication subscale), and quality of life (bodily pain domain). Small effect sizes were observed for increase in two quality of life domains: role limitations due to physical problems, and energy/fatigue.

A combination of quantitative and qualitative methods, including participant feedback, were used to determine that CBT-I was safe, acceptable, and feasible to administer among PWH. Participants shared their perceptions of CBT-I, which were used to better understand pre-existing needs of PWH, the positive impact of CBT-I, perceived mechanisms of change, challenges, and suggested modifications to make CBT-I more effective for PWH. 


\section{Discussion}

While the extant research has examined sleep disturbance among PWH, this is the first study to date to do so using American Academy of Sleep Medicine guidelines for evaluation of chronic insomnia (Schutte-Rodin et al., 2008). This is also the first study to examine the impact of CBT-I, the gold standard treatment for insomnia (Qaseem et al., 2016; Schutte-Rodin et al.) among a sample of PWH. The sample included a wide range of PWH in terms of age, education level, and employment status. There was less variability, however, in sex, gender, sexual orientation, ethnicity, and annual income, with the sample being more heavily composed of White, gay, male, lower-income individuals. Relative to Canadian HIV prevalence data, this sample underrepresents several groups, including women and Indigenous individuals, who compose $23.4 \%$ and $11.3 \%$ of PWH in Canada, respectively (Public Health Agency of Canada, 2018). This sample is not representative of the broader population of PWH in Toronto, as only participants who met criteria for insomnia disorder were included. Therefore, inferences about prevalence of sleep disturbance among PWH cannot be made. Rather, this study describes characteristics of sleep disturbance among PWH with insomnia.

\section{Features of Insomnia}

One goal of the present study was to examine features of insomnia among a sample of PWH with DSM-5 insomnia disorder, including comorbid disorders and psychological covariates. This was only the second study to date to examine sleep disturbance among PWH using a prospective sleep diary, and the first to do so using data collected over the recommended two-week monitoring period (Schutte-Rodin et al., 2008). The only other study to include sleep diary measurement of sleep disturbance among PWH (Lee et al., 2012) examined data collected over three nights. 
The sample was composed of individuals with chronic insomnia, with most participants having experienced insomnia for years. Subjective insomnia severity was subthreshold to moderate for almost all participants, with less than $10 \%$ of participants rating their insomnia as severe. In terms of specific types of sleep disturbance, prospective two-week sleep diary data identified that a greater proportion of participants were classified as poor sleepers based on wakefulness after sleep onset than they were based on sleep onset latency. This is also consistent with extant literature that suggests that a larger proportion of PWH report problems with sleep maintenance than with sleep onset (Allavena et al., 2016).

Sleep characteristics. With respect to cutoffs differentiating good versus poor sleepers based on sleep onset latency and wakefulness after sleep onset, over half of participants were classified as poor sleepers in terms of falling asleep, and three quarters in terms of staying asleep, with only a handful of participants classified as good sleepers on both of these criteria. Sleep efficiency was also a problem for the majority of participants, with the two-week average sleep efficiency in the sample falling below the $85 \%$ cutoff used to differentiate good sleepers from those with insomnia, and $83 \%$ of participants scoring below this value. Participants spent, on average, almost two and a half hours awake per night.

CBT-I treatment targets. In planning for the subsequent pilot study of CBT-I, the presence of potential treatment targets was examined. Potential targets include daytime napping, extended time in bed (e.g., getting into bed early; sleeping in), engaging in non-sleep activities in the bedroom, and experiencing worry, anxiety, and/or physical tension when attempting to sleep (Smith \& Perlis, 2006). While the mean length of daytime napping was under 30 minutes, there was large variability in nap time, with values ranging from 0 minutes to 2 hours and 51 minutes. 
With a median daytime napping value of 13 minutes, problematic daytime napping does not appear to be a relevant treatment target for most PWH in this sample.

Excessive time in bed was identified as a problem, with participants spending almost nine hours in bed. Extended time in bed diminishes sleep drive and creates more opportunity for people to associate the bed and bedroom with non-sleep activities, thereby contributing to conditioned arousal, a contributing factor to the maintenance of insomnia (Perlis, Giles, Mendelson, Bootzin, \& Wyatt, 1997; Riemann et al., 2010). Engaging in non-sleep activities is another specific treatment target, however, this was not quantified in the current study. During baseline assessments, however, participants reported engaging in non-sleep activities in bed, including using computers or smart phones to watch videos, read the news, or stay connected with friends, or family, watching television, and reading. When unable to fall asleep, participants reported worrying and/or planning for the upcoming day.

Experiencing worry, anxiety, and/or physical tension when attempting to sleep is another treatment target, as it also contributes to conditioned arousal. Pre-sleep arousal in this sample is consistent with that reported in other samples with insomnia disorder, and is elevated relative to that of poor sleepers without a diagnosis of insomnia disorder, and normal sleepers (JanssonFröjmark \& Norell-Clarke, 2012). Although participants reported mild levels of depression, anxiety, and stress, these were approximately twice as high as those reported in a similar sample of HIV-positive men who have sex with men in Australia without insomnia (Landstra et al., 2013). This may suggest that PWH with insomnia experience greater depression, anxiety, and stress relative to those without insomnia. Therefore, experiencing worry, anxiety, and/or physical tension when attempting to sleep was considered to be a relevant treatment target in the current sample. 
Of the potential CBT-I treatment targets identified by Smith and Perlis (2006), extended time in bed, engaging in non-sleep activities in the bedroom, and experiencing worry, anxiety, and/or physical tension when attempting to sleep were identified as relevant for this sample of PWH. Caution is warranted in identifying population-level treatment targets, as every population is heterogeneous, and each individual will present with their own combination of treatment targets. Annual income, education level, and employment status varied widely in the current sample, as did primary sleep complaint (e.g., sleep onset versus sleep maintenance), which may contribute to different perpetuating factors and treatment targets. CBT-I, perhaps more so than CBT protocols for other disorders, merits an idiographic approach to treatment and treatment planning, given that several different processes impact sleep. A focus on the wrong process in treatment, for example, sleep drive rather than conditioned arousal for a patient whose primary perpetuating factor for insomnia is conditioned arousal, may result in ineffective therapy at best, or iatrogenic harm at worst. Careful case conceptualization and treatment planning is an important feature of CBT-I.

Comorbidity. Psychiatric comorbidity was high among this sample, with over half of participants meeting criteria for at least one non-sleep psychiatric disorder. This rate is consistent with literature on psychiatric comorbidity among $\mathrm{PWH}$, which reports that $33-50 \%$ of individuals meet criteria for a least one mental disorder (Klinkenberg \& Sacks, 2004). Social anxiety disorder, alcohol use disorder, and major depressive disorder were the most common, followed by generalized anxiety disorder and substance use disorder. This, too, is consistent with the literature, which reports social anxiety disorder and generalized anxiety disorder as the most common anxiety disorders among PWH (Klinkenberg \& Sacks). The rate of depression in the current study, $12.5 \%$, was toward the lower end of prevalence estimates among people living 
with HIV, which are frequently reported at between 10-20\% (Klinkenberg \& Sacks, 2004;

Ohayon, 2007). This relatively lower rate of depression was surprising, given the already elevated rate of depression among PWH, and that individuals with insomnia are 2.27 times more likely to be depressed ( $\mathrm{Li}, \mathrm{Wu}, \mathrm{Gan}, \mathrm{Qu}, \& \mathrm{Lu}, 2016)$. Given that participants were required to self-refer to the study, it is possible that the current sample disproportionately represents nondepressed PWH, or at least those with less severe or interfering depression symptoms, who were motivated to participate in a research study.

With regard to sleep-related psychiatric disorders, OSA was the most common aside from insomnia disorder. Just over half of participants were identified as likely having OSA based on DSISD screening criteria. Surprisingly, literature on OSA among PWH is almost nonexistent. There are currently no epidemiological estimates of OSA prevalence among PWH. This is despite the presence of several biological and pharmacological risk factors for OSA development among PWH, including 1) increased incidence of upper airway infections, which lead to enlarged adenoid and tonsils, 2) lipodystrophy, or fat redistribution, which is one of the side effects of cART, which may result in fat deposits around the neck, thorax, and abdomen, making breathing more difficult, 3) weight gain due to sedentary lifestyle and/or medication side effects, and 4) potentially impaired upper airway neuromuscular control due to HIV-related inflammatory cytokine production (Goswami, Baker, Wang, Khalil, \& Kunisaki, 2015; Taibi, 2013). There was a high rate of suspected OSA identified among participants at baseline (33.3\%), and confirmed or suspected OSA was the most common exclusion criteria for the study, (35\% of those excluded). Overall, among all participants initially screened via telephone, approximately $25 \%$ were identified as high-risk for experiencing OSA, either during telephone screen or baseline assessment. As mentioned previously, only $63.1 \%$ of eligible participants 
completed the baseline assessment, therefore, it is possible that the prevalence of OSA may have been even higher if all eligible participants had participated in the study. OSA is a serious health problem affecting PWH, and more research is needed on its prevalence and impact among this population, as current literature is scant.

Physical health problems were common, and interfered with recruitment and testing. Of the 36 eligible participants who did not end up participating, 25\% cited medical or personal issues for withdrawing. Of the eleven participants to initiate CBT-I, one (9\%) withdrew after one session, also citing medical reasons for withdrawing. Of those participants who completed the baseline assessment, bothersome HIV-related symptoms were associated with lower cART adherence, reduced quality of life in areas of physical health, emotional wellbeing, social functioning, pain, and role limitations due to emotional problems, greater dysfunctional beliefs about sleep, sleep effort, pre-sleep arousal, depression, anxiety, and stress. Furthermore, the number of bothersome HIV-related symptoms was also associated with a greater number of comorbid psychiatric disorders. Commonly reported physical symptoms, affecting at least half the sample, were fatigue, muscle/joint pain, changes in body appearance, bloating, sexual problems, and skin problems such as itching and dryness. Several participants receiving CBT-I reported experiencing cold- or flu-like illness during the study, which increased the length of treatment.

Psychological covariates. Subjective insomnia severity was associated with poor mental health, unhelpful attitudes and beliefs about sleep, and social functioning-related quality of life. Specifically, greater insomnia severity was associated with greater depression, anxiety, stress, sleep effort, and worry/helplessness about sleep, and lower sleep self-efficacy and social functioning-related quality of life. While causality was not examined in the current study, and 
cannot be inferred here, these associations are consistent with theoretical models of insomnia, including cognitive (Espie, Broomfield, MacMahon, Macphee, \& Taylor, 2006; Harvey, 2002) and hyperarousal (Perlis et al.,1997; Riemann et al., 2010) models. Cognitive models propose that worrying, rumination, and unhelpful thinking, whether about general problems or sleep disturbance and its functional impact, leads to selective attention at bedtime that heightens anxiety and exacerbates sleep difficulty, perpetuating insomnia (Schwartz \& Carney, 2012). Hyperarousal models highlight the role of conditioned arousal in perpetuating insomnia. Conditioned arousal occurs when, over time, the bed is associated with poor sleep, and the bed eventually becomes a conditioned stimulus for arousal and ongoing sleep difficulty (Bootzin, 1972).

As proposed in cognitive models of insomnia, dysfunctional beliefs about sleep may contribute to sleep disturbance, and are amenable to cognitive restructuring. The mean item score on the DBAS-16 was well above the cutoff of 3.8 that is associated with clinically significant insomnia, and also above the mean DBAS-16 score reported in one study among a sample of 114 individuals with insomnia and medical comorbidity $(M=4.27, S D=1.47$; Carney et al., 2010). This suggests that dysfunctional beliefs about sleep are prevalent among PWH with insomnia, and possibly even relative to other individuals with insomnia and medical comorbidity, however, more research is needed in order to draw comparisons between PWH and people without HIV with insomnia.

Sleep self-efficacy was moderate in the sample, with the average score falling just under halfway between "not at all" and "very" confident on a visual analog scale. Participants, therefore, did not report very high or low self-efficacy, on average, with the mean score leaning slightly more toward low versus high efficacy. Variability was high for sleep self-efficacy in this 
sample. Therefore, although the mean score was moderate, a large number of participants scored both high and low on sleep self-efficacy. Sleep effort was also moderate in the sample, with the mean score falling just over half way between the minimum and maximum scores, within the range corresponding to the response option "to some extent" to questions of sleep effort. Presleep arousal was also moderate in the sample. While there are no recommended cutoff scores for the PSAS, the mean score fell within the range corresponding to the response option "slightly" to questions of how intensely participants experience various aspects of arousal as they attempt to fall asleep.

Overall, depression, anxiety, and stress were mild in severity, which was unexpected, given the high rates of depression typically observed among PWH (Klinkenberg \& Sacks, 2004) and among people with insomnia (Ohayon, 2007). As mentioned previously, this may be due to the nature of this study protocol which required participants to 1) proactively respond to advertisements, 2) complete a two-week sleep diary, and 3) attend an in-person appointment, in order to participate. This recruitment and screening process may have resulted in a sample with less severe and interfering psychopathology than might be observed among their nonparticipating peers.

In terms of quality of life, only social functioning was associated with insomnia severity in this sample, while energy/fatigue-, pain-, and general health-related quality of life were not. Reduced quality of life in the social domain may be disproportionately associated with insomnia severity over other domains, given the pervasive and potent impact of HIV stigma on the lives of many PWH. Despite efforts to reduce HIV stigma since the beginning of the pandemic, HIV remains a stigmatized illness, with many $\mathrm{PWH}$ fearing and/or facing discrimination in relationship, occupational, and social domains (Whetten, Reif, Whetten, \& Murphy-MacMillan, 
2008). Indeed, PWH may be less likely to have secure employment and greater poverty than their HIV-negative counterparts (Liu, Canada, Shi, \& Corrigan, 2012). In the current sample, only $23 \%$ reported having full time employment, whereas $45.9 \%$ were unemployed or on long term disability. The financial impact of unemployment and underemployment is reflected in the current sample, with $44 \%$ earning less than $\$ 20,000 /$ year, placing them below the poverty line in Ontario. The combination of HIV stigma, un-/underemployment, and poverty may impact social functioning. The subset of participants who completed CBT-I and the exit interview described feeling disengaged from society across social, occupational, and financial domains. They drew explicit links between this disengagement and their sleep difficulty, noting how isolation, boredom, lack of structure, and poverty all contributed to their insomnia. Although social functioning was not the quality of life domain with the lowest rating, participants explicitly linked it to their sleep difficulty during exit interviews.

\section{HIV and Insomnia}

HIV-related variables such as CD4 count and viral load were not directly associated with indicators of insomnia. Given the sample size, indirect associations were not examined. The average CD4 count among this sample of PWH with insomnia was within the normal range, and only $21 \%$ of participants reported having a CD4 count below 350 cells $/ \mu \mathrm{L}^{3}$. CD4 count was predictably positively associated with cART adherence, and was also negatively associated with fatigue. There was no evidence in the current study that CD4 count was associated with any indicators of insomnia or mediators of CBT-I. Evidence for the role of CD4 count in insomnia has been mixed in previous studies of PWH, with older studies prior to the advent of cART demonstrating such an association (Brown et al., 1991; Darko et al., 1992), but more recent studies reporting no association (Cohens et al., 1996, Nokes \& Kendrew, 2001; Robbins et al., 
2004). More recent evidence has demonstrated an association between lower CD4 count and more specific parameters of sleep disturbance such as number of awakenings (Lee et al., 2001), greater sleep fragmentation (Lee et al., 2012), suggesting that the association between CD4 count and sleep disturbance is highly nuanced.

HIV viral load was reported as undetectable among almost $88 \%$ of participants, therefore no further analyses were performed using this variable. This rate of viral suppression is consistent with rates reported elsewhere, both in Ontario (Burchell et al., 2015) and internationally (Boender et al., 2015).

A greater number of participants rated their cART medication adherence favourably on a single-item measure of adherence (SRSI) than was identified using a composite dichotomous measure (SMAQ). Whereas $93.8 \%$ reported their adherence as either excellent or very good, just under half of participants were identified as being cART adherent. Greater cART adherence, as measured by the single-item measure, was associated with fewer nighttime awakenings and bothersome HIV-related symptoms, and, predictably, higher CD4 count. To infer that improving cART adherence may indirectly improve insomnia via reduction in number of awakenings and bothersome symptoms would be premature without further research, as there is no evidence of a causal relationship. To further complicate this issue, the composite measure of cART adherence was associated with sleep efficiency, such that sleep efficiency was almost $10 \%$ higher among nonadherent than adherent participants. This finding may suggest a role of cART in maintaining insomnia among PWH, however, this association was not observed with a second measure of cART adherence, and no group differences in insomnia were observed among participants using different cART regimens. The role of antiretroviral medication in insomnia is complex, given that different regimens are composed of different classes of medication. Despite evidence in the 
literature that antiretroviral medications have been associated with sleep disturbance, including, but limited to, efavirenz (Gallego et al., 2004; Kenedi \& Goforth, 2011), there was no evidence in the current study that specific cART regimens were associated with indicators of insomnia, including Atripla, which contains efavirenz as a component.

Two HIV-related variables, time since HIV diagnosis and number of bothersome HIVrelated symptoms, were associated with a number of proposed mediators of CBT-I. A slight majority of participants, $66.7 \%$, reported that HIV diagnosis either predated or coincided with insomnia onset. It is unclear to what extent HIV diagnosis and/or infection played a causal role in the development of insomnia among PWH, as this was beyond the scope of the present study. It is clear, however, that for approximately one third of participants, insomnia was a problem prior to HIV diagnosis. After controlling for age, longer time since HIV diagnosis was associated with greater self-efficacy for sleep and lower sleep effort. There was no association between time since HIV diagnosis and subjective insomnia severity or any other sleep parameters.

Older age was independently associated with a number of positive outcomes relative to younger age, including greater quality of life related to energy/fatigue and emotional wellbeing, fewer comorbid psychiatric conditions, lower depression severity, less cognitive arousal at bedtime, and fewer dysfunctional beliefs about sleep. This is consistent with literature reporting higher prevalence and incidence of depression among younger versus older PWH in Ontario (Choi, Boyle, Cairney, Collins et al., 2016). Older versus younger age was associated with one negative outcome, lower quality of life related to physical functioning. These results suggest that over time, PWH may form more adaptive attitudes toward living with insomnia, and adapt to adverse circumstances, which speaks to the resilience of this population in the face of adversity. This also suggests that people more recently diagnosed with HIV, but more specifically those 
younger in age, may be in greater need of psychological support than their older peers and/or those who have lived with HIV longer. As noted above, this is consistent with findings from a large sample of PWH in Ontario (Choi et al.).

The most commonly reported bothersome HIV-related symptoms were fatigue/loss of energy, difficulty sleeping, muscle aches/joint pain, anxiety, bloating, depression, changes in body appearance, sexual problems, memory problems, and skin problems. While the number of bothersome HIV-related symptoms was not directly associated with indicators of insomnia, it was associated with dysfunctional beliefs about sleep, sleep effort, and pre-sleep arousal, and as such, may play a role in perpetuating insomnia. Furthermore, number of bothersome HIV-related symptoms was associated with psychological distress, including number of comorbid psychiatric disorders, greater depression, anxiety, and stress, poorer cART medication adherence, and lower quality of life across a number of domains. Given the association between number of bothersome symptoms and proposed mediators of CBT-I, and the modifiable nature of many of these symptoms, introducing strategies to manage these symptoms may improve the efficacy of CBT-I among PWH. For example, cognitive restructuring, which is already a component of CBT-I, could also be used to manage depression, anxiety, and unhelpful beliefs associated with body changes and sexual functioning.

CBT protocols have been tailored to target health concerns relevant to $\mathrm{PWH}$, including cART side effects (Doerfler \& Goodfellow, 2016), cART adherence and depression (CBT-AD; Safren et al., 2016), methamphetamine use and cART adherence (Parsons, John, Millar, \& Starks, 2018), pain management (Trafton et al., 2012), trauma and self-care (O'Cleirigh et al., 2019), body image and self-care (Blashill et al., 2017), alcohol use (Papas et al., 2010), discrimination-related coping (Bogart et al., 2018), and sexual health promotion (Hart et al., 
2016). Broadening the scope of cognitive restructuring during treatment to target other relevant concerns may provide added benefit to $\mathrm{PWH}$, and is consistent with feedback from participants in the exit interview.

Fatigue was a problem for participants, and was elevated relative to reported levels of fatigue among PWH without insomnia (Johansson et al., 2014). Fatigue was not directly associated with indicators of insomnia or any proposed mediators of CBT-I. In terms of HIVrelated variables, fatigue was associated with lower CD4 count, less time since HIV diagnosis, and greater number of bothersome HIV-related symptoms. It was associated with greater depression, anxiety, and stress, as well as poorer quality of life in all domains except physical functioning and bodily pain. While fatigue was a problem for PWH with insomnia, it does not appear to be directly associated with insomnia or mediators of CBT-I. More research is needed to explore potential indirect associations between fatigue and insomnia among PWH.

\section{CBT-I Pilot Study}

This was the first study to examine the impact of CBT-I among PWH. Intervention effects were examined, along with safety, acceptability, and feasibility.

Intervention effects. At the group level, subjective insomnia severity reduced from moderate at baseline to no insomnia at posttreatment, and a large effect size was observed. While this study design did not include a control group or randomization to provide a basis for interpreting causal role of CBT-I, it made use of interrupted time-series single-case design for this purpose, which demonstrated that reliable change in both magnitude and slope of insomnia severity was observed for seven of the ten participants, while reliable change on either magnitude or slope was observed for all participants. Furthermore, reliable change in insomnia severity occurred only after CBT-I was initiated for each participant, which supports a causal 
role of CBT-I. Sustained reliable change was observed after just one session for $40 \%$ of participants, and within three sessions for $80 \%$. The reduction in insomnia severity was clinically significant for all but one participant. These results suggest that CBT-I is effective at reducing insomnia severity among PWH. Symptom change intercorrelation revealed that as insomnia severity decreased, so did sleep effort, depression, anxiety, and stress, and there was an increase in fatigue/energy-related quality of life. These results are consistent with participant feedback from the exit interview, that CBT-I was helpful at improving sleep to varying degrees. While all participants reported improvement, some reported rapid and complete remission, whereas others reported ongoing room for improvement.

At the group level, sleep efficiency increased from $65 \%$ to $89 \%$ from baseline to posttreatment, and a large effect was observed. Reliable change in magnitude of sleep efficiency was observed for all but one participant, and with a slightly more relaxed criterion of reliable change $(\geq 2$ SD rather than $>2$ SD), reliable change was observed for all participants. Reliable change in sleep efficiency occurred only after CBT-I was initiated, thus supporting a causal role of CBT-I. Sustained reliable change occurred after just one session of CBT-I for $70 \%$ of participants. The target sleep efficiency in CBT-I ranges from $85 \%$ to $90 \%$, with lower scores indicative of ongoing sleep difficulty and higher scores indicative of excessive sleepiness and sleep deprivation, but not insomnia. At posttreatment, target sleep efficiency was observed for just two participants, with five surpassing the target range with sleep efficiency $>90 \%$. CBT-I protocol is to gradually increase the time in bed prescription when sleep efficiency is $>90 \%$ and sleep onset latency is $<15$ minutes, such as instructing the individual to add 15 minutes more to the time in bed prescription per week until the target sleep efficiency is reached. Given that half the sample continued to have elevated sleep efficiency $>90 \%$ at posttreatment despite gradually 
increasing their time in bed prescriptions, this population may benefit from more aggressive time in bed increases, additional sessions, or a few follow-up appointments in person or via telephone to assist with time in bed adjustments. These results suggest that CBT-I is effective at increasing sleep efficiency, but that PWH may require additional follow-up to ensure that sleep efficiency does not remain above the target range. Alternatively, undiagnosed OSA may also have contributed to persistently elevated sleep efficiency, and is discussed in more detail below. Symptom change intercorrelation revealed that as sleep efficiency increased, total wake time, sleep onset latency, and number of awakenings also decreased, which is not surprising, given that sleep efficiency is calculated based on time asleep versus awake in bed.

A large effect was observed for decrease in total wake time, which reduced from just over 3 hours per night at baseline to 44 minutes at posttreatment. Reliable change was observed for all but one participant at posttreatment. A longer follow-up period may have demonstrated reliable change for the remaining participant, as the value indicative of reliable change ( $>2 \mathrm{SD}$ below baseline) was observed at just the final time point, but not the final two consecutive time points. Sustained reliable change in total wake time occurred only after CBT-I was initiated, and occurred after just one session for $70 \%$ of participants. These results suggest that CBT-I is effective at reducing total wake time among PWH, and is also consistent with qualitative feedback indicating that CBT-I was helpful at improving sleep.

Change in viral load was not examined, as no participants reported having a detectable viral load at baseline. Literature on the association between viral load and sleep disturbance is sparse and equivocal, with support for an association between viral load and sleep quality (Saberi et al., 2011) as well as support for no association (Robbins et al., 2004). In Canada, the most recent estimate is that $81 \%$ of people diagnosed with HIV are receiving cART, and $91 \%$ of those 
receiving cART have suppressed viral load (Public Health Agency of Canada, 2018). As initiatives continue to reach the joint United Nations Programme on HIV/AIDS and World Health Organization 90-90-90 target of having 90\% of people with HIV diagnosed, $90 \%$ of those diagnosed receiving cART, and 90\% of those on cART reaching viral suppression (Public Health Agency of Canada), examining the impact of viral load on sleep may become increasingly challenging and potentially less relevant.

CD4 count was within the normal range of 500 cells $/ \mu \mathrm{L}^{3}$ to 1,600 cells $/ \mu \mathrm{L}^{3}$ for individuals with healthy immune functioning (Public Health Agency of Canada, 2016) at baseline and posttreatment, and the small increase over time was neither statistically nor clinically significant. Furthermore, CD4 count was only collected at two time points, therefore, interrupted time-series single case design was not appropriate for examining this variable, and no causal relationship can be inferred. The literature is mixed on the association between CD4 count and sleep disturbance, with several studies reporting no association (Cohen et al., 1996; Nokes \& Kendrew, 2001; Robbins et al., 2004), and others reporting an association between CD4 count and only very specific features of sleep disturbance such as number of awakenings (Lee et al., 2001) and sleep fragmentation (Lee et al., 2012). In the current study, CD4 count was not associated with any sleep-related variables; however, a larger sample size with greater variability in CD4 count may be required to explore this association. Symptom intercorrelation revealed that as CD4 count increased, so did total sleep time and social functioning-related quality of life; however, causality cannot be inferred.

It is unclear whether cART adherence improved over the course of CBT-I, as the two measures used to assess adherence provided conflicting results. Results from the single-item adherence measure indicated that the proportion of participants with excellent adherence 
increased from $70 \%$ at baseline to $100 \%$ at posttreatment. Results from the more stringent composite adherence measure, however, indicated that just $40 \%$ of participants were adherent at baseline, and that this proportion decreased to $30 \%$ at posttreatment. Missing data on some of the items contributing to the composite measure may have resulted in an overestimation of the number of nonadherent participants. In the absence of complete data on the composite measure, the single-item measure may provide a more reliable estimate of adherence. The results, therefore, suggest that cART adherence improved from baseline to posttreatment; however, given that it was measured at only two time points, the study design precludes attribution of this improvement to CBT-I. Future research should examine the causal role of CBT-I in improving cART adherence by incorporating a control group into the study design.

While sample size precluded multivariate analysis of the role of covariates, effect sizes were calculated to determine the magnitude of difference between baseline and posttreatment scores. With one exception, bodily pain-related quality of life, change was in the desirable direction. Large effects were observed for a number of sleep-related variables, including time in bed, number of awakenings, wakefulness after sleep onset, and terminal wakefulness after sleep onset. Large effects were also observed for psychological variables such as sleep effort and selfefficacy for sleep.. A large effect was also observed for fatigue. Medium effect sizes were observed for number of bothersome HIV-related symptoms as well as sleep-related variables of sleep onset latency, total sleep time, and napping, and the psychological variable of cognitive arousal at bedtime. Finally, small effect sizes were observed for stress, depression, and quality of life related to role limitations due to: physical functioning, energy/fatigue, and bodily pain.

Surprisingly, pain-related quality of life decreased from baseline to posttreatment. This surprising result was further explored by examining correlations between symptom change 
scores, and the only statistically significant correlation with the decrease in pain-related quality of life was an increase in quality of life associated with role limitations due to emotional problems, $r=-.70, p=.024$. Although this does not imply a causal role, it is possible that as participants learned strategies to manage emotional problems, they became more engaged in activities, resulting in greater opportunity for pain flares and associated impact on quality of life in that domain. Feedback in the exit interview confirmed that participants felt better in the daytime, and were more active and productive, which is consistent with this hypothesis. While they mentioned a sense of living in a damaged body as a pre-existing need, no participants reported an increase in pain as an unwanted treatment outcome. It is possible that increases in pain, while reported on self-report questionnaires, were not significant, distressing, or impairing enough for participants to make note of them during the exit interview. While it is possible that these effects may be attributed to CBT-I, further research is needed using an experimental design such as a randomized controlled trial to determine causality. The goal of the current study was to demonstrate preliminary effects of CBT-I on several variables including insomnia severity, sleep efficiency, and total wake time, and to explore the safety, acceptability, and feasibility of CBT-I with PWH.

These results support four of the six hypotheses, that participants would experience a reduction in insomnia severity, improvement in sleep efficiency into the target range of $85 \%$ to $90 \%$, reduction in total wake time, and improved cART medication adherence. The second hypothesis was partially supported, as although participants experienced an improvement in sleep efficiency, the final sleep efficiency was not within the target range of $85 \%$ to $90 \%$ for all participants. Unlike goals for reducing insomnia severity and total wake time, the goal for sleep efficiency is to achieve complete remission, which is a more stringent goal. One hypothesis was 
not supported by the results, that participants would experience an increase in CD4 count. Finally, one hypothesis was not tested, that participants with a detectable viral load at baseline would experience a reduction in viral load, given that no participants reported a detectable viral load at baseline. Taken together, the results suggest that CBT-I is an effective treatment for insomnia among PWH. Furthermore, sustained change in sleep efficiency and total wake time occurred after just one session of CBT-I for $70 \%$ of participants, and preceded sustained change in insomnia severity, which occurred after just one session for only $40 \%$ of participants, and by the third session for $80 \%$. Elevated sleep efficiency above the target range at posttreatment suggests that PWH may require greater increases in time in bed prescription and/or a few extra sessions to ensure that sleep efficiency does not remain above $90 \%$. These results are consistent with qualitative participant feedback. While medium to large effect sizes were observed for a number of sleep-related and psychological variables, it remains unclear whether these changes can be attributed to CBT-I. Future research should incorporate a control group to determine whether CBT-I leads to improvement in these variables.

Safety. Another goal of this study was to examine the safety, acceptability, feasibility and participant perceptions of CBT-I using both quantitative and qualitative methods. Ensuring that a treatment is safe to administer is an essential step prior to widespread dissemination. While CBT-I is considered a safe treatment (Manber et al., 2014), this is the first time that it has been administered to $\mathrm{PWH}$, therefore, examining its safety with this population was essential. Using a mixed methods approach, including participant interview, therapist observation, and symptom review, it was determined that CBT-I is safe to administer to PWH. No adverse events were reported by participants or observed by the therapist. The only safety concern identified based on symptom review was elevated sleep efficiency $>95 \%$ in one participant, as mentioned above. 
Elevated sleep efficiency is typically associated with excessive daytime sleepiness, which poses a risk for accidents while driving or operating dangerous machinery or equipment. Precautions were taken to minimize this risk, such as taking short naps to restore alertness, ensuring that the participant did not drive or operate dangerous machinery while sleepy, and continued to increase time in bed to further reduce sleep efficiency over time. Excessive daytime sleepiness is possible with any population receiving CBT-I due to sleep restriction recommendations, and therapists must apply sleep restriction judiciously and monitor sleep efficiency and daytime sleepiness in order to ensure that CBT-I is safely administered to each individual. Overall, CBT-I was determined to be a safe treatment for PWH.

Acceptability. Mixed methods were also employed to explore acceptability of CBT-I, including participant interview, therapist observation, and self-report questionnaire. All participants, without exception, reported that CBT-I had improved their sleep and that they would recommend it to others. They also noted that no changes were needed, and that the CBT-I strategies and study administrative procedures were acceptable. Treatment credibility was examined via self-report questionnaire, and participants rated CBT-I as credible after the first session, and even more so at posttreatment after having received the full treatment. Two specific components of treatment credibility that increased from session one to posttreatment were confidence in treatment success at eliminating insomnia and confidence in recommending the treatment to a friend. Treatment adherence was measured via therapist observation and rating at each session, and was based on the degree to which the therapist felt that participants had implemented strategies and recommendations from the previous session, including following the time in bed prescription. Adherence, ranging from $0 \%$ to $100 \%$, was $64.8 \%$ across all sessions, and excluding session one at which adherence is based solely on sleep diary completion, 
therapist-rated adherence gradually improved from session two $(50.3 \%)$ to posttreatment (63.2\%). Attrition was very low, with only one participant withdrawing from the study after receiving the first session. This participant's data, as explained above, were excluded from analysis, given the pragmatic nature of the study; however, the attrition rate, including this single non-completer, is $9.1 \%$, which is lower than the attrition rates reported among other samples including $34.2 \%$ in a primary care mental health clinic offering low-intensity individual mental health treatment (Grant et al., 2012), 36\% in a trial of CBT-I among veterans (Karlin, Trockel, Spira, Taylor, \& Manber, 2015), and 45.9\% in a sample of PWH receiving CBT for peripheral neuropathy (Davis, Evans, Fishman, Haley, \& Spielman, 2004). Not all participants initially offered CBT-I chose to participate, with two participants preferring a less time-consuming option that did not provide treatment for insomnia, and other declining due to concern over PTSD symptomology. It is possible that despite experiencing insomnia, some individuals may not be interested in committing the time, work, and energy into participating in CBT-I. This is consistent with feedback from exit interviews that highlighted the importance of motivation and willingness to work hard in successful implementation of CBT-I. Overall, the strong participant endorsement, treatment credibility, adequate adherence to recommendations, and low attrition suggest that CBT-I is an acceptable treatment for PWH, but that motivation and cost-benefit analysis may play a role in who pursues, and benefits from, CBT-I.

Feasibility. Feasibility was examined in terms of the research protocol as well as administration of CBT-I. With regard to the research protocol, it was challenging to compose a sample of PWH with insomnia without OSA given the high prevalence of OSA in this population. Even with telephone screening to exclude individuals with OSA, it was identified as likely present among one-third of participants who completed the baseline assessment. There are 
several biological and pharmacological risk factors for OSA development among PWH (Goswami et al., 2015; Taibi, 2013), yet very little research has been published on OSA among PWH. While this may pose a challenge for researchers looking for samples of PWH without OSA, it also highlights an important health problem among PWH that merits further research and treatment. For purposes of ecological validity, future research should include PWH with OSA, while examining its association with insomnia. While literature examining the efficacy of CBT-I among individuals with comorbid OSA is scant, there is evidence that individuals with and without OSA benefit equally from standard CBT-I in terms of improvement in insomnia severity, sleep diary parameters, and daytime functioning up to three months posttreatment, and that it may be beneficial to treat insomnia prior to initiating CPAP therapy for OSA (Sweetman, Lack, Lambert, Gradisar, \& Harris, 2017). Thus, the high prevalence of OSA among PWH need not pose as a barrier to CBT-I, but may necessitate alterations to standard CBT-I protocol, such as more careful monitoring of sleepiness and use of sleep restriction (Sweetman et al.).

Recruitment progressed more slowly than anticipated, and three main reasons for this are proposed. First, the high prevalence of OSA among PWH resulted in a higher number of telephone screens in order to obtain the desired sample size. Relaxing exclusion criteria to include individuals with OSA would improve recruitment feasibility for future studies, and would increase ecological validity of the research, as mentioned above. Second, recruitment via PWA, and specifically their newsletter, was the most successful recruitment strategy, but was not used until late in the recruitment process. Aligning with HIV community organizations from the study onset would likely accelerate the recruitment process and increase feasibility of the research protocol. Third, all telephone screenings, baseline assessments, scheduling, follow-up communication, and therapy sessions were conducted by the primary researcher up until the final 
few weeks of the study when a research assistant was trained to conduct telephone screenings. This method was time-consuming, so distributing these tasks among a greater number of people would greatly increase recruitment feasibility.

With regard to CBT-I itself, the session structure and treatment recommendations were all feasible to administer, and as mentioned above, were acceptable to participants. The one aspect of CBT-I that was not feasible with this sample was strict adherence to a 4-session biweekly treatment schedule. Illness, school, work, and travel plans necessitated more flexible scheduling, including more time between sessions and occasionally adding extra sessions to review and/or consolidate material before proceeding further. While overall, CBT-I is feasible to administer to PWH, consideration should be made for events that may necessitate a more flexible treatment schedule, such as health problems.

In sum, the pragmatic pilot study demonstrated that CBT-I reduces insomnia severity, improves sleep efficiency, and reduces total wake time among PWH. Adherence to cART, as well as a number of other sleep-related and psychological covariates improved over the course of treatment; however, causal relationships were not established for these variables. Furthermore, CBT-I is a safe, acceptable, and feasible treatment for insomnia among PWH.

\section{Participant Perceptions of CBT-I}

Participants expressed several pre-existing needs prior to receiving CBT-I, which were unrelated to their sleep disturbance, and which are typically not addressed in CBT-I. They referenced the idea of living in damaged bodies due to HIV or other health problems, noting the impact of this on their sleep. They described widespread psychological distress among their community of PWH, including feelings of grief and loss. Finally, they expressed feeling disengaged from society socially, occupationally, and financially, noting that this interfered in 
their ability to fully implement treatment recommendations. In Ontario, just half of all PWH with depression are accessing mental health services, and of those, only $51 \%$ were receiving services aligned with Canadian guidelines. Furthermore, access to mental health services was lower among PWH who identified as gay, lesbian, or bisexual, those low income and education, and immigrants and those who spoke English as a second language (Choi, Boyle, Cairney, Gardner, et al., 2016). Mental health services are underutilized among PWH in Ontario, and access needs to be improved, especially for certain disadvantaged subgroups. Furthermore, incorporating HIV-specific content into CBT-I, which was recommended by several participants, may also serve to address the sense of disengagement from society.

Participants described several positive impacts of the treatment, three of which corresponded to pre-existing needs they had described. All participants reported that their sleep improved, with several expressing surprise at how quickly and completely their sleep disturbance had resolved. Improvements were noted in quality, consistency, duration, and number of awakenings. Participants reported feeling more informed, and that the educational component of CBT-I was helpful at correcting misconceptions about sleep and providing strategies that made sense and were effective. Participants reported that the process of receiving CBT-I had resulted in self-improvement more broadly, in addition to improving their sleep. For example, they reported feeling more confident in their ability to face problems and cope with adversity, as well as feeling more in control and more organized. Some participants noted that implementing CBTI strategies such as avoiding napping and scheduling activities during the daytime had given them a sense of purpose and helped them to re-engage with society. As participants gained confidence and more effective coping skills, they reported feeling more well-equipped to engage with others, whether at work or socially. This positive impact addressed the pre-existing need of 
feeling disengaged from society. Several participants noted feeling less groggy and having more energy in the daytime, which had a positive impact on daytime activity level. This addressed preexisting concerns about living in a damaged body. Finally, participants reported greater psychological wellbeing after receiving CBT-I. Several participants mentioned that anxiety decreased considerably, with participants making sense of this in different ways. One participant attributed his sleep improvement to reduced anxiety and another attributed his reduced anxiety to sleep improvement. Other participants mentioned that applying cognitive restructuring strategies in areas other than insomnia improved mood and ability to cope with other stressors. This addressed pre-existing concerns about psychological distress. The positive impact of CBT-I described by participants addressed all of the pre-existing needs that had also been identified, and is therefore useful for PWH above and beyond the impact that it has on insomnia. Even without modifications, CBT-I appears to address pre-existing needs among PWH to some extent, and incorporating participant feedback to tailor CBT-I may even further increase its usefulness for PWH beyond the scope of treating insomnia.

Participants attributed the positive impact of CBT-I to several factors, which were grouped into two overarching categories during thematic analysis: general factors and CBT-I strategies. The general factors participants identified as impacting treatment outcome are the relationship they developed with the therapist, the therapist's skill and approach in providing CBT-I, and the importance of their own motivation to engage in therapy. The relationship with the therapist was important for most participants. Specifically, they mentioned the importance of the therapist being warm, approachable, interested in them, and being able to understand the issues and experiences they're facing. The therapist skills they appreciated included being able to provide a rationale for each recommended strategy, being knowledgeable about CBT-I, being 
patient, encouraging participants to make difficult, but necessary changes. Most of these general factors are not specific to CBT-I, but are qualities that contribute to favourable therapy outcomes. Common factors that are associated with medium effect sizes on psychotherapy outcome include goal consensus/collaboration, empathy, alliance, positive regard/affirmation, and genuineness (Wampold, 2015). The general factors that patients in the current study found helpful correspond with the common factors that are known to positively impact psychotherapy outcome. The fact that so many participants highlighted the importance of the therapeutic relationship is consistent with the pre-existing need of feeling disengaged from society, and may be particularly relevant for a population that has long experienced discrimination and stigmatization from society and health care providers alike. The benefit of a strong therapeutic relationship may lead to more than just the anticipated reduction in insomnia severity, to include increased self-esteem and confidence, which were in fact both reported as positive impacts of CBT-I in the current study.

Participants also believed that treatment success depends on one's level of motivation, in addition to factors related to the therapist and treatment strategies. This insight reflects an essential component of any behavioural change intervention - a client's readiness to change. A client who is not ready to make difficult changes, such as those integral to CBT-I, may require additional assistance in increasing readiness for change. Motivational interviewing is a communication style that decreases ambivalence and builds motivation to change (Miller \& Rollnick, 2002). In CBT-I, this may take the form of completing a cost-benefit analysis of behavioural change versus maintaining the status quo when a client is having difficulty implementing behavioural strategies such as adhering to a standard wake-up time or avoiding daytime napping (Manber \& Carney, 2015). It is important with CBT-I, as it is with other 
therapy involving behaviour change, to ensure that participants are motivated and ready to make the necessary changes before proceeding any further. In the current study, similar monetary incentives were offered for both the single two-hour visit and the much lengthier time commitment of ten weeks to receive CBT-I, so participants who were motivated to participate by the monetary incentive rather than the opportunity to resolve their insomnia may have viewed CBT-I as not worth the cost in time commitment. PWH are at greater risk of poverty than their HIV-negative counterparts (Liu et al., 2012), and this may have influenced decisions to forego CBT-I in favour of more immediate monetary incentive. In fact, one CBT-I participant did request, and was granted, part of the incentive provided in advance in order to purchase food. For future research or implementation of CBT-I in community settings, it may be useful to guide individuals through a cost-benefit analysis in the form of a decisional balance worksheet, weighing the pros and cons of change versus the pros and cons of maintaining the status quo, thereby increasing motivation to take part in CBT-I and/or adhere to difficult treatment recommendations.

Factors specific to CBT-I that participants identified as helpful include thinking differently about sleep, getting out of bed when awake, avoiding napping, and developing structured routines. These represent both cognitive and behavioural components of CBT-I. Patients came to recognize the self-fulfilling prophecy of worrying about sleep, and appreciated cognitive restructuring strategies for thinking in more helpful ways at bedtime and also during the day. Several participants reported balanced thinking that helped them to accept occasional sleep disturbances rather than worry about the consequences. Participants emphasized the importance of using the bed only for sleep and sex, and even then, only spending the allotted amount of time in bed. A lot of participants spent considerably more time bed than they were 
able to sleep for, and they found that matching the time in bed with their individual sleep need was an important strategy. Although CBT-I recipients typically end up developing routines around bed and wake times, this particular group of PWH found that developing structured routines resulted in significant and widespread benefits in many domains. Participants who were not currently working described having minimal structure and few routines, noting that this may have played a role in developing insomnia. For example, without a reason to wake up at a certain time any day of the week, and without a reason not to nap during the daytime, both sleep drive and circadian rhythm are impacted by extended time in bed, daytime napping, and inactivity. Many participants, including ones working full-time jobs, reported benefitting from adding structure to their daily and nightly routines. CBT-I may be particularly beneficial for PWH who are unemployed, given the emphasis on developing routines and increasing activity level. This feature of CBT-I also addresses disengagement from society, and participants made this link explicit in providing feedback. For example, one participant reported treating CBT-I like the job he was encouraged to give up after being diagnosed with HIV, leading to a sense of daily purpose. Another participant explicitly stated that although he was currently unemployed, he hoped to soon find work, and developing daily routines in advance was an important and helpful part of that process.

Participants reported several challenging aspects of CBT-I, and most were associated with implementing strategies they found the most useful. These particular challenges included learning to dispute unhelpful thoughts, completing the sleep diary, and adhering to sleep restriction and stimulus control guidelines of resisting naps, waking at the prescribed time, and getting out of bed when unable to sleep. HIV-related factors such as medication side effects, fatigue, and lack of structured routines made it more difficult for participants to adhere to sleep 
restriction recommendations. Another challenge was adhering to strategies that participants felt negatively impacted important relationships with partners and pets, such as resisting social activities to maintain a sleep schedule that they found helpful. For a group who, in the current study, identified disengagement from society as a problem, maintaining social connections is crucial. Given that the most useful strategies were also the most challenging, therapists providing CBT-I to PWH should take special care to identify and decrease any barriers to these strategies early on in therapy. The high prevalence of undiagnosed, but likely OSA in this sample may also explain why participants had particular difficulty adhering to sleep restriction and stimulus control guidelines including resisting naps, waking at the prescribed time, and getting out of bed when unable to sleep. OSA results in daytime sleepiness (Chung et al., 2012), which may make the above recommendations even more difficult to adhere to.

When asked for feedback on how CBT-I could be modified to better meet the needs of PWH, participants provided several helpful recommendations. Not all participants believed that their insomnia was associated with HIV infection, but those who did requested more information on this association. Participants were informed during the recruitment process that insomnia was more prevalent among PWH, and they sought answers as to why that is. They noted a lack of information from other health care providers on how HIV and insomnia may be linked, and a subset of participants expressed great interest in learning more about this. Several participants had expressed feeling as though they were living in a damaged body, therefore, accurate information on the association between HIV and insomnia may serve to demystify some of the ways in which they feel that HIV has caused that damage.

Participants suggested incorporating technology into the treatment and administrative procedures, such as being able to access questionnaires online, complete an electronic version of 
the sleep diary (which one participant did using a spreadsheet), and using text communication rather than telephone and email. These suggestions reflect a desire to make CBT-I and study administrative procedures more convenient, which is important for a population that experiences barriers in the form of poverty and discrimination (Liu et al., 2012; Shacham et al., 2015; Whetten et al., 2008). Participants recommended adding content to address what they perceived to be prevalent issues among $\mathrm{PWH}$, including reducing substance use and learning assertive communication skills. In fact, some participants noted that treatment recommendations sometimes conflicted with their personal goals and/or schedules; but did not express these concerns to the therapist during treatment. The two participants who reported keeping these concerns to themselves completed treatment with sleep efficiency above the target range, and assertive communication may have improved treatment outcomes for these specific participants. This illustrates the importance of incorporating assertive communication strategies into CBT-I for PWH. Not only would assertive communication improve therapy outcomes for select individuals, but it would also facilitate communication with friends, partners, landlords, employers, health care providers, etc., and decrease social isolation. Participants also requested that session content be made more explicit in advance to provide a roadmap for therapy. Participants pointed out questionnaire items and response options they found unhelpful, and one participant recommended modifying the sleep diary to track timing of HIV medication and nonalcohol substance use. Tracking medication and substance use other than alcohol and sleep medication may provide patients with insight regarding the impact of these substances on sleep; however, this information could be captured in the "comments" section rather than modifying the standard sleep diary. Finally, participants requested more therapy, and recommendations included adding another two sessions for a total of six, spending more time on some or all 
sessions, spending more time practicing specific strategies, and making CBT-I more widely accessible for PWH, which is consistent with the pre-existing need of psychological distress, and with literature reporting that mental health services are underutilized among PWH (Choi et al., 2016).

Despite offering suggestions for making CBT-I more acceptable and effective for PWH, almost all participants noted that changes were not actually needed in order for it to be helpful. They found the CBT-I strategies acceptable, noting that they were able to overcome challenges they initially faced, including completing the sleep diary and following sleep restriction and stimulus control recommendations. They found CBT-I to be helpful, practical, and sustainable. They also found the administrative procedures acceptable. All participants reported that they would recommend this treatment to a friend with HIV and insomnia, and indeed, some of them already had. Overall, participants communicated that CBT-I was an acceptable treatment for insomnia for PWH.

Participant feedback affirmed that CBT-I was helpful at improving, and in many cases, resolving, insomnia. This is consistent with quantitative results that CBT-I was effective in decreasing insomnia severity and total wake time, and increasing sleep efficiency for the majority of participants. Feedback also suggested that cognitive and behavioural strategies were helpful not only for improving sleep, but also for addressing pre-existing needs of PWH by fostering overall self-improvement and a greater sense of wellbeing in the daytime, and also by reducing depression and anxiety symptoms for some participants. The strategies participants found most helpful were learning to think differently about their sleep, getting and/or staying out of bed when they were awake, avoiding napping, and developing structured routines. These strategies correspond with CBT-I components of cognitive restructuring, stimulus control, and 
sleep restriction, respectively. There were no safety concerns reported by participants, and feedback suggested that CBT-I, in its standard format, is an acceptable treatment for insomnia for PWH. Soliciting qualitative feedback is essential when piloting a treatment with a new population, as participant reactions and perspectives cannot be captured by self-report questionnaires. Participant feedback is even more important when working with vulnerable populations such as PWH, given the history of discrimination and HIV stigma (Shacham et al., 2015; Whetten et al., 2008). To conclude that CBT-I need not be tailored for PWH given feedback on its acceptability, helpfulness, and sustainability in its standard form would be to further propagate the sense of disengagement experienced by PWH. Tailoring CBT-I may serve to meet even more unmet needs and empower a community that has historically faced considerable discrimination (Shacham et al., 2015).

\section{Tailoring CBT-I for PWH}

While the standard CBT-I protocol was effective at reducing insomnia severity among this sample of PWH, and participants found this protocol acceptable, they provided some recommendations on tailoring CBT-I to better meet the needs of PWH. The following recommendations, summarized in Figure 38, are drawn from patient feedback as well as therapist observation during the study and results of the exit interview thematic analysis.

Administrative aspects. In this small sample of PWH, flexibility in scheduling was essential, given a number of factors that impacted participants' ability to attend appointments at regular biweekly intervals. Factors included illness, vacation, and work and school schedules. The diversity in income and employment status in the sample resulted in different scheduling needs, with full-time employed participants requiring more flexibility in scheduling than their retired or unemployed peers; however, this may also be true of other populations. Participants 


\begin{tabular}{|c|c|}
\hline Category & Recommendations \\
\hline Administrative Aspects & $\begin{array}{l}\text { - Flexible scheduling } \\
\text { - Appointment reminders } \\
\text { - Use of technology for greater convenience }\end{array}$ \\
\hline CBT-I Protocol & $\begin{array}{l}\text { - Two optional sessions for adding content, adjusting sleep window, or practicing strategies } \\
\text { - Close monitoring of daytime sleepiness } \\
\text { - Less rigorous sleep restriction in the presence of suspected/diagnosed obstructive sleep apnea } \\
\text { - Small group format } \\
\text { - Administered by staff in HIV community organizations trained in CBT-I }\end{array}$ \\
\hline Additional Content & $\begin{array}{l}\text { - Decisional balance exercise at treatment outset on pros and cons of change versus status quo } \\
\text { - Psychoeducation on association between HIV and insomnia } \\
\text { - Psychoeducation on importance of developing structured routines } \\
\text { - Psychoeducation on role of alcohol and substance use on sleep } \\
\text { - Handout outlining what to expect in treatment } \\
\text { - Assertive communication training } \\
\text { - Fatigue and pain management strategies }\end{array}$ \\
\hline
\end{tabular}

Figure 38. Recommendations for tailoring cognitive behavioural therapy for insomnia (CBT-I) for people living with HIV. 
with relatively unstructured schedules benefitted from appointment reminders, and given the large proportion of participants in the current study who were unemployed, retired, or on longterm disability, appointment reminders may be useful to incorporate into the administrative aspect of CBT-I for PWH. Some participants requested more technological innovation such as texting about appointment scheduling or being able to complete questionnaires and sleep diary online.

CBT-I protocol. Some participants found the standard four-session protocol to be sufficient; however, others requested more therapy. Quantitative results are also equivocal in this regard, with insomnia severity reducing after one session for several participants, but not until the third session for others. PWH may not require more than the standard four sessions across the board, but some may benefit from extra time learning and practicing the strategies and finetuning the time in bed prescription. Given that some participants requested more time working on strategies they learned in therapy, it may be beneficial to offer PWH four to six sessions of CBT-I, with the goal at the outset being to provide treatment in four sessions, with the option to add an additional two sessions, if sleep efficiency is not in the target range and the participant has been adherent to treatment. If new content (see below) is added to the standard CBT-I protocol to benefit PWH, more sessions would be required. The new content would be optional depending on case conceptualization.

The elevated sleep efficiency at posttreatment may suggest that participants, even those who attained rapid clinically significant reduction in insomnia severity, may benefit from more time in therapy adjusting their sleep window. An alternative to adding more sessions would be to take a more aggressive approach to increasing the time in bed prescription for $\mathrm{PWH}$, as these results demonstrate that while participants did adhere to their gradually increased (e.g., 15 
minutes/week) time in bed prescriptions, sleep efficiency remained elevated. This persistent elevated sleep efficiency observed in around half of participants may reflect underlying OSA in this population, however. OSA results in daytime sleepiness (Chung et al., 2012), so increasing the time in bed prescription may not lower sleep efficiency as it would among individuals without OSA. In administering CBT-I to individuals with suspected OSA, sleepiness should be closely monitored for safety purposes, and sleep restriction should be relaxed to account for greater time in bed requirements (Sweetman et al., 2017). Achieving a sleep efficiency in the range of $85 \%$ to $90 \%$ may be difficult in the presence of OSA, as sleep efficiency may remain higher than desired once insomnia remits. Furthermore, realistic expectations should be set regarding improvements in sleep quality and daytime functioning in the presence of untreated OSA, and individuals should be encouraged to seek treatment upon completion of CBT-I.

Another modification to the CBT-I protocol that may prove beneficial for PWH would be to offer it in a small group format with up to six participants per group. The efficacy of group CBT-I has been established (Koffel, Koffel, \& Gehrman, 2015; Norell-Clarke, JanssonFröjmark, Tillfors, Hollandäre, \& Engström, 2015), and there are multiple potential benefits to group CBT-I. Specific to this population, one benefit is the opportunity for participants to engage with peers and form meaningful relationships, which addresses the disengagement from society identified as a pre-existing need. Another benefit is that group CBT-I would likely be more accessible for PWH, given its more cost- and time-effective format. Participants had requested that CBT-I be more accessible for them and their peers, such as being offered for free or at low cost through a community organization such as ACT or PWA. This would be most feasible if it were offered in group format by interdisciplinary practitioners (e.g., psychologists, clinical social workers, counselors, nurses, physicians, psychiatrists, etc.) working in community organizations 
who are trained to administer CBT-I. As mentioned above, one barrier to offering CBT-I in group format for this population is the flexibility in scheduling that is required. This barrier may be reduced by selecting group members based on preferred schedules (daytime vs evening). General benefits of group CBT-I not specific to this population include peer support and problem-solving, and greater adherence to treatment recommendations when success is observed among other members (Manber \& Carney, 2015).

Additional content. In terms of content, guiding participants through a decisional balance exercise at treatment outset may increase motivation, something identified by participants as essential to treatment success. Adding psychoeducation on the role of HIV (disease and medication) in insomnia, including what remains unknown, would be helpful for PWH. Psychoeducation on the importance of developing structured routines, both for improving sleep and for overall wellbeing would be useful. A roadmap of what to expect in each session would also be helpful, based on participant feedback, and could be provided as a handout during the first session. Other content either recommended by participants or identified via thematic analysis is psychoeducation on the role of alcohol and substance use in sleep disturbance and assertive communication skills training. This content would benefit select participants based on case conceptualization, and could therefore be offered in optional sessions five and six if needed, or, in a group CBT-I format, be incorporated into the six-session group protocol. Fatigue was a problem for most participants, and made it more difficult to adhere to sleep restriction recommendations. It is therefore important to address in therapy to the extent that it is identified as a barrier for specific individuals during attempts to increase sleep drive through increasing activity in the daytime or decreasing napping and/or lingering in bed. Bothersome HIV-related symptoms, including fatigue and chronic pain were highly prevalent and were associated with 
proposed mediators of CBT-I such as dysfunctional beliefs about sleep, sleep effort, and presleep arousal. Therefore, introducing strategies to manage these symptoms and/or highlighting how cognitive restructuring strategies could be applied to these other areas may improve treatment efficacy. Some participants recommended modifying the sleep diary to include timing of non-sleep medication and other substances. Given that the CSD (Carney et al., 2012) is a standardized diary, a more feasible strategy would be to instruct PWH to include this information in the comments section if they are interested in observing how medications and substances affect their sleep pattern.

\section{Limitations}

There are several limitations to the current study. A convenience sample composed of self-selected participants was used, therefore, the sample is not representative of the population of PWH in Toronto, and less so of PWH more broadly, thus limiting the generalizability of the results. There were very few female-identified participants in the study, which also limits generalizability. One reason for this may be that in Canada, diagnostic rates for males are three times higher than for females, with $75.2 \%$ of reported HIV cases in 2017 being among males (Haddad, Li, Totten, \& McGuire, 2017). With specific regard to the CBT-I pilot study, however, this sample is more likely to approximate treatment-seeking PWH in the community, as it is composed of individuals who saw and responded to advertisements seeking individuals with difficulty sleeping. The CBT-I pilot study, therefore, has ecological validity that may compensate for the larger study's lack of generalizability. CBT-I requires a collaborative approach with individuals already motivated to overcome their insomnia, and treatment-seeking individuals, such as the ones represented in this study, are the subpopulation of PWH who would 
benefit from CBT-I. Another limitation is the small sample size which precluded the use of many potentially useful quantitative analyses due to low power.

CBT-I was administered to all participants after the same number of time points in the baseline observation phase. This small number of baseline observations, while meeting the minimum number required for this type of design, may not have resulted in an accurate representation of baseline symptoms for some participants whose two or three scores appeared to demonstrate a trend during the baseline phase. Trends during the baseline phase in the same direction as that predicted by the treatment complicate evaluation of intervention effects (Kazdin, 1982). To compensate for this, multiple evaluation methods were used, including the twostandard deviation band method, change in slope, latency of change, and $t$-tests and effect sizes for combined pre/post scores. Using a longer baseline observation period with more time points would improve confidence in the stability of insomnia symptoms during the baseline phase, and provide further support for a causal role of CBT-I in improving insomnia severity, sleep efficiency, and total wake time among PWH. On a similar note, all participants received CBT-I after the same number of baseline observations. Initiating CBT-I after different numbers of baseline observations across participants would also improve confidence in the causal role of CBT-I.

There was no long-term follow-up to determine whether treatment effects were maintained posttreatment. While this study demonstrated reduction in insomnia severity at posttreatment, it is unknown whether insomnia symptoms returned in the weeks and months after treatment ended, and whether participants continued to implement strategies learned during CBT-I. Other limitations of the pilot study design include lack of a control group and lack of independent assessment. Given that this is the first study to examine the effects of CBT-I among 
PWH, a full randomized controlled trial would have been premature, therefore, a control group was not necessary in the current study, but would be an important next step for future research examining efficacy of CBT-I among this population.

Finally, another limitation is that the primary researcher (T. Tulloch) was also the baseline assessor, sole study therapist, and one of two researchers who conducted the thematic analysis of exit interviews. While this multi-role situation resulted from the nature of the current study as a dissertation research project with limited financial and personnel resources, it has the potential to introduce bias into several phases of the project, including participant selection, preand post-treatment assessment, and interpretation of results. To minimize bias in the participant selection process, communication was standardized using a scripted telephone interview with predetermined inclusion/exclusion criteria. To minimize bias in the assessment process, eligibility was determined based on predetermined criteria gathered via semi-structured interview and prospective self-report data from the sleep diary. To minimize bias in interpretation of the results, participants completed computerized questionnaires at posttreatment, an independent assessor administered the exit interviews, and the thematic analysis was conducted by T. Tulloch along with another researcher not involved in the study (C. Mutschler). Furthermore, visual aids were applied to minimize bias in conducting visual analysis of primary outcomes. Although the above measures cannot guarantee elimination of all bias, attempts were made to minimize the impact of researcher bias through all stages of the research project.

\section{Conclusions}

This is the first study to examine sleep disturbance among PWH using American Academy of Sleep Medicine guidelines for evaluating chronic insomnia (Schutte-Rodin et al., 2008), and is also the first study to examine the impact of CBT-I among a sample of PWH. Sleep 
disturbance is highly prevalent among PWH (Smith et al., 2005), therefore, this study represents an important step toward better understanding and treating insomnia in this population with unique needs. This study presented characteristics of insomnia among PWH based on self-report questionnaires, semi-structured interviews, and two-week prospective sleep diaries which document chronic insomnia of moderate severity, with difficulty staying asleep being more common than difficulty falling asleep and waking up too early. Relevant CBT-I treatment targets were identified in the sample, including daytime napping, extended time in bed, engaging in nonsleep activities in the bedroom, and experiencing worry, anxiety, and/or physical tension at bedtime. Psychiatric comorbidity was common, as were comorbid sleep disorders, with OSA being the most prevalent. Insomnia severity was associated with sleep effort, self-efficacy for sleep, depression, anxiety, stress, and social functioning-related quality of life. Surprisingly, cART medication regimen was not associated with insomnia severity. Almost $80 \%$ of participants reported CD4 count within the normal range for individuals with healthy immune functioning, and almost $90 \%$ reported undetectable viral load.

The CBT-I pilot study demonstrated, through single-case interrupted time-series design, that CBT-I reduced insomnia severity, increased sleep efficiency, and reduced total wake time. Large effect sizes were observed for each of these results. Clinically significant reduction in insomnia severity was observed for all but one participant, and occurred relatively quickly, with reliable change observed among four out of ten participants after just one session, and another four participants by the third session. Sleep efficiency improved for all participants, but remained below the target cutoff for three participants, and exceeded the cutoff for another five. This suggests that participants may have benefitted from more time adjusting the time in bed prescription to ensure that sleep efficiency reached the target range by posttreatment. It may also 
reflect undiagnosed OSA in this sample, which may lead to ongoing daytime sleepiness regardless of sleep duration. Exit interviews provided valuable feedback on the pre-existing needs of PWH prior to therapy, perceived positive impacts of CBT-I, perceived mechanisms of change, challenges faced by participants, and suggested modifications to the standard CBT-I protocol to make it more helpful for PWH. Participant feedback also demonstrated that CBT-I is a safe and acceptable treatment for PWH, and it was also determined to be feasible to administer to this population.

\section{Implications and Future Directions}

Several issues were identified that could help guide future research and treatment of insomnia among PWH. OSA was highly prevalent among individuals screened for this study, therefore, composing future samples of PWH without OSA may prove challenging. Recent research suggests that the presence of OSA may not impact CBT-I efficacy (Sweetman et al., 2017), therefore, future research should attempt to include individuals with OSA for ecological validity. Extra precaution should be taken to monitor daytime sleepiness and implement sleep restriction more judiciously among individuals with comorbid insomnia and OSA. Furthermore, the prevalence and impact of OSA among PWH is a topic that has received insufficient attention in the literature, and merits further research.

The finding that older age, and to a lesser extent time since HIV diagnosis, is associated with a number of optimal mental health outcomes suggests that younger PWH may benefit from CBT-I and other psychological interventions to a greater extent than their older peers. Given the social isolation reported by some of the participants in the exit interview, a peer support group where older PWH share their experiences and coping strategies with younger PWH may be mutually beneficial. This type of intervention could potentially be offered via HIV/AIDS 
community organizations such as ACT that offer a wide range of programming and services for PWH and people at risk of contracting HIV.

The prevalence of depression in the current sample was surprisingly low, given epidemiological estimates of depression among PWH (Klinkenberg \& Sacks, 2004; Ohayon, 2007), and the greater likelihood of depression among individuals with insomnia (Li et al., 2016). This may reflect a genuine low prevalence of depression among PWH in Toronto, or it may indicate that this self-referring sample of PWH with insomnia did not capture the subset of individuals with depression who may not have been motivated to participate in research study, or who may not have seen the advertisements in the first place. Future research should attempt to recruit PWH with depression and insomnia in order to document characteristics of insomnia and provide much-needed treatment to this group, and to compose samples more representative of the PWH community.

The heterogeneity in this sample highlights the importance of taking an idiographic approach to CBT-I through crafting of each case conceptualization and careful selection of appropriate interventions. While most participants in this study reported bothersome HIV-related symptoms that were associated with proposed mediators of CBT-I, issues such as fatigue were not as relevant for some participants as for others. While CBT-I is based on a standardized protocol (Edinger \& Carney, 2008), it is recommended that case conceptualization be used to determine which components to administer, and in what order (Manber \& Carney, 2015). This holds true for PWH, who may have a number of pre-existing needs unique to a population living with a stigmatized illness, including feeling disengaged from society and living with numerous HIV- and medication-related physical symptoms that may impact administration of CBT-I. 
Finally, future research examining insomnia among PWH should seek to recruit larger samples in order to increase the statistical power for quantitative analyses. Given that this was the first study to examine the impact of CBT-I among a sample of PWH, it would have been premature to conduct a full randomized controlled trial. Now that there is preliminary evidence for the effectiveness of CBT-I at reducing insomnia severity among PWH, future research could examine the efficacy of CBT-I using a randomized control group design, and further examine mediators of treatment efficacy. Larger sample size could be achieved by partnering with community organizations such as ACT and PWA where PWH seek services and have established relationships. Another important future goal is to make CBT-I more accessible to PWH, perhaps by offering small group format through HIV/AIDS community organizations, and/or training clinical social workers and counsellors to administer CBT-I at those sites.

This study provides preliminary evidence for the efficacy of CBT-I among PWH, however, more research is needed to examine its efficacy relative to control conditions and to examine mechanisms of treatment efficacy. PWH have typically been excluded from CBT-I research, given the disproportionately high prevalence of insomnia among this population and the possibility that unique factors not amenable to change may be playing a role. This study demonstrates that insomnia among PWH is amenable to change via standard CBT-I, thereby paving the way for PWH to be included in future CBT-I research. 
Appendices

Appendix A

\section{Exit Interview Questions}

Overall aim: Elicit feedback that can be used to optimize CBT-I for people living with HIV

Objective 1: Gain a more complete picture of the impact of CBT-I on sleep-and HIV-related outcomes, as well as any other unintended outcomes that might pertain to the safety of CBT-I among this specific population.

1. How has this program affected your sleep?

2. How has this program affected your life in ways other than your sleep?

3. Were there any negative consequences for you as a result of participating in this study?

4. At the end of treatment, your therapist reviewed your progress with you. What do YOU think about your progress?

5. Did you use marijuana for medical purposes during this study? (If yes, probe: How did that affect your sleep? How did that affect your overall progress?)

Objective 2: Explore participants' knowledge about the CBT-I strategies presented during treatment and the extent to which they found each strategy helpful, in order to determine whether specific strategies are more or less helpful for people living with HIV.

6. If your insomnia were to return in the future, what advice would you want give your future self to help overcome it?

7. What about this program was the most useful for you?

8. Here is a list of some strategies for managing insomnia. Can you tell me which of these were the most helpful for you? (Probe: Why was that strategy helpful?)

9. Which strategies weren't as helpful for you? (Probe: Why was that strategy not helpful?)

10. Using that same list, can you tell me about some of the challenges you faced when trying each of these strategies? (Probe: Anything else?)

Objective 3: Elicit feedback to determine if and how CBT-I could be specially tailored to make it as acceptable and effective as possible for people living with HIV.

11. If you could change something about the therapy sessions to make them more helpful for people living with HIV, what would you change? (Probe: What else would you change?)

12. What did you think about your homework exercises and handouts?

a. What could be changed to make the homework exercises and handouts more helpful for people living with HIV? (Probe: What else?)

13. What did you think about your therapist?

a. What could he have done differently to make the treatment more helpful for you?

14. Thinking about any other parts of the study, including the consent form, assessments, and scheduling, what could be changed to make this study more convenient?

15. What are some of the things you liked the most about this experience? (Probe: Anything else?)

16. If you had a friend with both HIV and insomnia, would you recommend this treatment to them?

a. What would you tell them?

17. Were you disappointed with any part of this program or its results? (Probe: What were you disappointed about?)

18. Can you think of anything that might prevent someone with HIV from benefitting from this treatment? 
Appendix B

Study Measures

\section{Socio-Demographic Questionnaire}

Welcome to the POSITIVE SLEEP STUDY!

Please DO NOT include your name anywhere in this questionnaire. Please answer each question honestly and to the best of your ability. Again, since you are completely anonymous, do not feel pressured to respond in a way that you "think you should". Just answer truthfully.

Thank you for taking the time to participate in our research! Your involvement is critical to research on insomnia among people living with HIV. The people involved in conducting this research are extremely grateful for your time, patience, and effort.

Please follow the guidelines on each of the following questionnaires.

Instructions: Here are some basic questions about YOU. Please do not attach your name to this or any other sheet. Remember, all of your answers are confidential and you can not be identified by any of the pieces of information you provide on this, or any other sheet in the questionnaire package.

Today's Date

Date of Birth

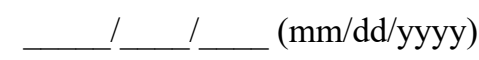

Age (in years)

First, we would like to ask you some information about your HIV status and other general background information.

What is your HIV status?

HIV-positive

HIV-negative

I do not know my HIV status

When were you first diagnosed with HIV (e.g., tested positive for HIV)?

Less than 6 months ago

6-12 months ago

1-5 years ago (2011-2015)

6-10 years ago (2006-2010)

11-15 years ago (2001-2005)

16-20 years ago (1996-2000)

21-25 years ago (1991-1995)

26-30 years ago (1986-1990)

Over 30 years ago (before 1986)

I cannot remember

I do not know

I prefer not to say 
Specifically, how many years ago were you first DIAGNOSED with HIV (e.g., tested positive for HIV)?

When was your most recent medical checkup involving a blood test?

Within the past 3 months

Between 3-6 months ago

Between 9-12 months ago

Between 1-2 years ago

Between 2-5 years ago

Over 5 years ago

I have never had a medical checkup involving a blood test

I cannot remember

What was your HIV viral load at your most recent medical checkup?

Undetectable

Less than 100,000 copies $/ \mathrm{mL}$

100,000 copies $/ \mathrm{mL}$ or higher

I was told my viral load, but I cannot remember

I was not told my viral load

Other (please specify):

What was your CD4+ count at your most recent medical checkup?

Less than $200 / \mu \mathrm{L}$

$200-350 / \mu \mathrm{L}$

over $350 / \mu \mathrm{L}$

I was told my CD4+ count, but I cannot remember

I was not told my CD4+ count

Other (please specify):

What term do you use to describe your gender? (Check all that apply)

Male

Female

Transman

Transwoman

Genderqueer/gender non-conforming

2-spirited

Other (please specify) 
If you had to choose one single term that you felt best described your gender, which would you choose?

Male

Female

Transman

Transwoman

Genderqueer/gender non-conforming

2-spirited

Other (please specify)

What sex were you assigned at birth, on your original birth certificate?

Male

Female

What terms do you use to describe your sexual orientation? (Check all that apply)

Gay or homosexual

Straight or heterosexual

Bisexual

Asexual

Pansexual

2-spirited

Queer

Other (please specify)

If you had to choose one single term that you felt best described your sexual orientation, which would you choose?

Gay or homosexual

Straight or heterosexual

Bisexual

Asexual

Pansexual

2-spirited

Queer 
Other (please specify)

What is your employment status?

Full time employed

Part time employed

Self-employed

Housewife/husband

Unemployed

Retired

Other (please specify)

What is the highest level of education you have reached/completed?

Did not attend high school

Some high school education

High school diploma

Some university, college or technical school education

Bachelor's degree, college diploma, or technical certificate

Some graduate or professional school

Graduated graduate or professional school

Are you currently a student?

Yes, full time

Yes, part time

No

Prefer not to answer

Are you currently earning money through paid work (legal or under the table)?

Yes

No

Prefer not to answer

In the past six months, have you received money from any of the following sources? (Check all that apply)

Provincial income assistance (welfare)

Employment insurance (EI or UIC)

Retirement pension income (public or private)

Student loans or grants 
Loans (other than student loans)

Gift or loan from partner, family member, or friend

Disability insurance/worker's compensation

Escort work or sex work

Selling drugs

Other legal sources of income

Other non-legal sources of income

I did not receive income from any of these sources in the past six months

Prefer not to answer

Annual income: Please indicate which of the following best represents your annual income from paid work and all other sources before taxes and deductions.

$$
\begin{aligned}
& \text { Under } \$ 10,000 \\
& \$ 10,000-\$ 19,999 \\
& \$ 20,000-\$ 29,999 \\
& \$ 30,000-\$ 39,999 \\
& \$ 40,000-\$ 49,999 \\
& \$ 50,000-\$ 59,999 \\
& \$ 60,000-\$ 69,999 \\
& \$ 70,000-\$ 79,999
\end{aligned}
$$

Over $\$ 80,000$

Religion: Please indicate the religion of which you were raised (Check all that apply):

Catholic

Protestant (e.g. United Church, Anglican, Lutheran, Presbyterian)

Evangelical Protestant (e.g. Baptist, Jehovah's Witness, Pentecostal, $7^{\text {th }}$ Day Adventist)

\section{Eastern Orthodox}

Christian - Other (Please specify)

Jewish

Islamic

Hindu

Sikh

Buddhist

Agnostic

Spiritual, but I do not believe in a God

None

Other (please specify) 
Please indicate the religion you currently practice (Check all that apply):

Catholic

Protestant (e.g. United Church, Anglican, Baptist, Lutheran, Presbyterian)

Evangelical Protestant (e.g. Baptist, Jehovah's Witness, Pentecostal, $7^{\text {th }}$ Day Adventist)

Eastern Orthodox

Christian - Other (Please specify)

Jewish

Islamic

Hindu

Sikh

Buddhist

Agnostic

Spiritual, but I do not believe in a God

None

Other (please specify)

Ethnic Background: What ethnicity do you identify with? (Check as many as apply to you):

African (e.g., Nigeria, Ghana, Ethiopia)

African-Caribbean (i.e., from the Caribbean and of Black/African descent)

Indo-Caribbean (i.e., from the Caribbean and of South Asian descent)

Other Caribbean (i.e., from the Caribbean and of other ethnic descent)

South Asian (e.g., India, Pakistan, Bangladesh, Sri Lanka)

East Asian (e.g., Hong Kong, China, Japan, Korea)

Southeast Asian (e.g., Singapore, Malaysia, Thailand, Cambodia, Philippines)

Middle Eastern or North African (e.g., Iran, Israel, Egypt, Morocco)

Latin American

Aboriginal/Métis/Inuit

White - British (e.g., England, Ireland, Scotland, Wales) 

White - French
White - Other European (e.g., German, Italian, Russian, Portuguese, etc.)
Mixed race/ethnicity
Other (please specify)

Ethnic Background: What single ethnicity do you MOST identify with?:

African (e.g., Nigeria, Ghana, Ethiopia)

African-Caribbean (i.e., from the Caribbean and of Black/African descent)

Indo-Caribbean (i.e., from the Caribbean and of South Asian descent)

Other Caribbean (i.e., from the Caribbean and of other ethnic descent)

South Asian (e.g., India, Pakistan, Bangladesh, Sri Lanka)

East Asian (e.g., Hong Kong, China, Japan, Korea)

Southeast Asian (e.g., Singapore, Malaysia, Thailand, Cambodia, Philippines)

Middle Eastern or North African (e.g., Iran, Israel, Egypt, Morocco)

Latin American

Aboriginal/Métis/Inuit

White - British (e.g., England, Ireland, Scotland, Wales)

White - French

White - Other European (e.g., German, Italian, Russian, Portuguese, etc.)

Mixed race/ethnicity

Other (please specify)

In which country were you born?

How long have you lived in Canada?

Which country were your parents born in?
Mother
Father

Which country do your parents live in?

Mother

Father 
What is your current immigration status (today)?

\author{
Canadian citizen \\ Permanent resident \\ Refugee/Refugee applicant \\ Temporary work permit \\ Student visa \\ Undocumented \\ Prefer not to say
}

Other (please specify):

In what language are you most comfortable communicating?

English

French

Spanish

Arabic

Mandarin

Cantonese

Punjabi

Tagalog

Korean

Italian

Greek

Portuguese

Russian

Other (please specify): 
What kind of housing do you live in now?

Apartment, condo, or house

Room in a rooming house/single room occupancy hotel

Student residence

Hospice or palliative care residence

Shelter or hostel

Treatment or recovery house (drug-use related)

No fixed address/homeless/couch-surfing/street

Other (please specify)

Please indicate who (if anyone) you live with (Check as many as apply to you)

By myself

Roommate(s)

Partner(s) or spouse(s)

Parent(s)

Grandparent(s)

Other Family Member(s) - [e.g. sibling(s), aunt(s), uncle(s), cousin(s)]

Child(ren)

Group or residential program

Other (please specify)

Please indicate who (if anyone) you share your bed with (check all that apply).

I sleep alone

Partner(s) or spouse(s)

Child(ren)

Other Family Member(s)

Roommate(s)

Pet(s)/animal(s) (please specify):

Other (please specify): 
What is your current relationship status? Please check off all that apply.

Single

Have a boyfriend(s)

Have a girlfriend(s)

Living with a male partner(s) for a year or more

Living with a female partner(s) for a year or more

Have a husband

Have a wife

Separated/Divorced/Widowed

What term best describes the gender of your primary partner?

Man (non-trans)

Woman (non-trans)

Trans man

Trans woman

Genderqueer/gender non-conforming

Two-spirit

Other (please specify)

Thank you for your responses so far. Next, we are going to ask you some questions about your sleeping patterns and your attitudes and beliefs about sleep. Please answer each question to the best of your ability. 
DBAS-16

Please indicate to what extent you personally agree or disagree with each statement by circling a number that indicates where your personal rating falls.

1. I need 8 hours of sleep to feel refreshed and function well during the day.

$\begin{array}{llllllllllll}\text { Strongly Disagree } & 1 & 2 & 3 & 4 & 5 & 6 & 7 & 8 & 9 & 10 & \text { Strongly Agree }\end{array}$

2. When I don't get the proper amount of sleep on a given night, I need to catch up on the next day by napping or on the next night by sleeping longer.

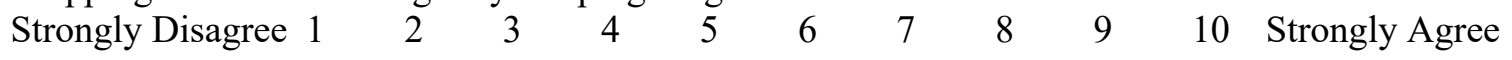

3. I am concerned that chronic insomnia may have serious consequences on my physical health.

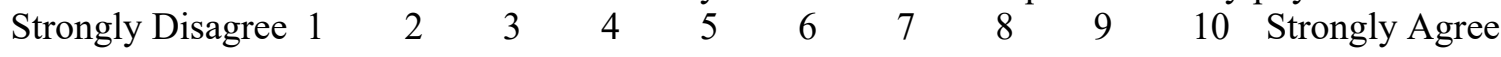

4. I am worried that I may lose control over my ability to sleep.

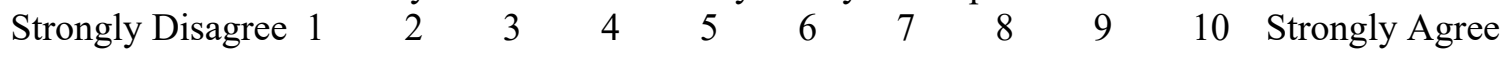

5. After a poor nights sleep, I know that it will interfere with my daily activities on the next day.

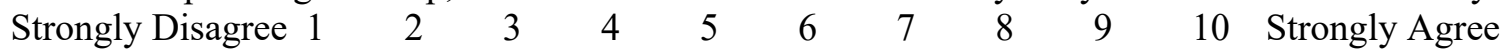

6. In order to be alert and function well during the day, I am better off taking a sleeping pill rather than having a poor night's sleep.

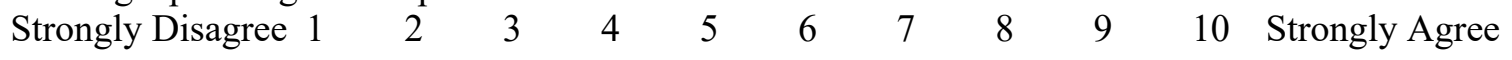

7. When I feel irritated, depressed, or anxious during the day, it is mostly because I did not sleep well the night before.

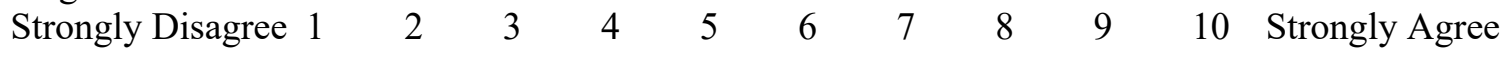

8. When I sleep poorly on one night, I know it will disturb my sleep schedule for the whole week. $\begin{array}{llllllllllll}\text { Strongly Disagree } & 1 & 2 & 3 & 4 & 5 & 6 & 7 & 8 & 9 & 10 & \text { Strongly Agree }\end{array}$

9. Without an adequate night's sleep, I can hardly function the next day.

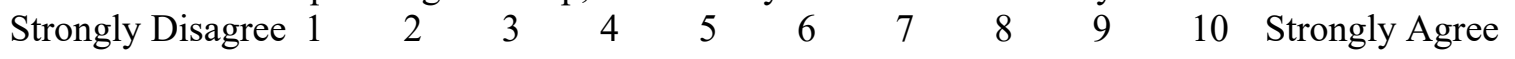

10. I can't ever predict whether I'll have a good night's sleep.

$\begin{array}{lllllllllllll}\text { Strongly Disagree } & 1 & 2 & 3 & 4 & 5 & 6 & 7 & 8 & 9 & 10 & \text { Strongly Agree }\end{array}$

11. I have little ability to manage the negative consequences of disturbed sleep.
Strongly Disagree 1
23
4
$\begin{array}{llll}5 & 6 & 7 & 8\end{array}$
$9 \quad 10$ Strongly Agree

12. When I feel tired, have no energy, or just seem not to function well during the day, it is generally because I did not sleep well the night before.

$\begin{array}{llllllllllll}\text { Strongly Disagree } & 1 & 2 & 3 & 4 & 5 & 6 & 7 & 8 & 9 & 10 & \text { Strongly Agree }\end{array}$

13. I believe insomnia is essentially the result of a chemical imbalance.
Strongly Disagree 1
2
4
$\begin{array}{lllll}5 & 6 & 7 & 8 & 9\end{array}$
10 Strongly Agree

14. I feel insomnia is ruining my ability to enjoy life and prevents me from doing what I want.

$\begin{array}{llllllllllll}\text { Strongly Disagree } & 1 & 2 & 3 & 4 & 5 & 6 & 7 & 8 & 9 & 10 & \text { Strongly Agree }\end{array}$

15. A "nightcap" before bedtime is a good solution to sleeplessness.

$\begin{array}{lllllllllllll}\text { Strongly Disagree } & 1 & 2 & 3 & 4 & 5 & 6 & 7 & 8 & 9 & 10 & \text { Strongly Agree }\end{array}$

16. It usually shows in my physical appearance when I haven't slept well.

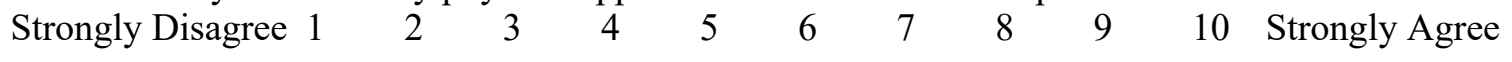




\section{GSES}

The following seven statements relate to your night-time sleep pattern in the past week. Please indicate by circling one response how true each statement is for you.

\begin{tabular}{|c|l|c|c|c|}
\hline 1 & $\begin{array}{l}\text { I put too much effort into sleeping when it should come } \\
\text { naturally }\end{array}$ & Very much & To some extent & Not at all \\
\hline 2 & I feel I should be able to control my sleep & Very much & To some extent & Not at all \\
\hline 3 & $\begin{array}{l}\text { I put off going to bed at night for fear of not being able to } \\
\text { sleep }\end{array}$ & Very much & To some extent & Not at all \\
\hline 4 & I worry about not sleeping if I cannot sleep & Very much & To some extent & Not at all \\
\hline 5 & I am no good at sleeping & Very much & To some extent & Not at all \\
\hline 6 & I get anxious about sleeping before I go to bed & Very much & To some extent & Not at all \\
\hline 7 & I worry about the consequences of not sleeping & Very much & To some extent & Not at all \\
\hline
\end{tabular}




\section{SES}

Instructions: Draw a vertical line through the point on the horizontal line beside each item to indicate how confident you feel in carrying out the behavior described in each item below.

1. Lie in bed, feeling physically relaxed.

Not at all

Very

Confident

Confident

2. Lie in bed feeling mentally relaxed.

Not at all

Very

3. Lie in bed with your thoughts "turned off."

Not at all

Very

4. Fall asleep at night in under 30 minutes.

Not at all

Very

5. Wake up at night fewer than 3 times.

Not at all

Very

6. Go back to sleep within 15 minutes of waking at night.

Not at all

Very

7. Feel refreshed upon waking in the morning.

Not at all

Very

8. Wake after a poor night's sleep without feeling upset about it.

Not at all

Very

9. Not allow a poor night's sleep to interfere with daily activities.

Not at all

Very 


\section{PSAS}

Please describe how intensely you generally experience each of these symptoms as you attempt to fall asleep in your own bedroom.

$\begin{array}{ccccc}1 & 2 & 3 & 4 & 5 \\ \text { not at all } & \text { slightly } & \text { moderately } & \text { a lot } & \text { extremely }\end{array}$

\section{Somatic}

1. Heart racing, pounding, or beating irregularly.

2. A jittery, nervous feeling in your body.

3. Shortness of breath or labored breathing.

4. A tight, tense feeling in your muscles.

5. Cold feeling in your hands, feet or your body

6. Have stomach upset (knot or nervous feeling, heartburn, nausea, etc.

7. Perspiration in the palms of your hands or other parts of your body.

8. Dry feeling in your mouth or throat.

\section{Cognitive}

9. Worry about falling asleep.

10. Review or ponder events of the day.

11. Depressing or anxious thoughts.

12. Worry about problems other than sleep.

13. Being mentally alert, active.

14. Can't shut off your thoughts.

15. Thoughts keep racing through your head.

16. Being distracted by sounds, noise in the environment, (e.g., ticking of the clock, house noises, traffic). 


\section{SRSI}

Please rate your ability to take your HIV medications over the past 4 weeks by circling one of the options below:

Very Poor Poor $\quad$ Fair $\quad$ Good $\quad$ Very Good Excellent

SMAQ

\begin{tabular}{|c|c|}
\hline 1. Do you ever forget to take your medicine? & YES \\
\hline 2. Are you careless at times about taking your medicine? & YES \\
\hline 3. Sometimes if you feel worse, do you stop taking your medicines? & $\begin{array}{l}\text { YES } \\
\text { NO }\end{array}$ \\
\hline 4. Did you miss any of your medications last weekend? & $\begin{array}{l}\text { YES } \\
\text { NO }\end{array}$ \\
\hline $\begin{array}{l}\text { 5. Think on the last week. } \\
\text { How often did you missed to take any medication? }\end{array}$ & $\begin{array}{l}\text { A: Never } \\
\text { B: } 1-2 \\
\text { C: } 3-5 \\
\text { D: } 6-10 \\
\text { E: More than } 10\end{array}$ \\
\hline $\begin{array}{l}\text { 6. How many days have you missed taking all your medicines during } \\
\text { the past } 3 \text { months? }\end{array}$ & Days:....... \\
\hline
\end{tabular}




\section{FSS}

Below are a series of statements regarding fatigue. By fatigue we mean a sense of tiredness, lack of energy, or total body give-out. Please read each statement and circle a number from 1 to 7 that indicates your degree of agreement with each statement where 1 indicates you strongly disagree and 7 indicates you strongly agree. Please answer these questions as they apply to the past week.

STRONGLY

DISAGREE
STRONGLY

AGREE
a. My motivation is lower when I am fatigued....... 1
2
3

4

5
6
7
b. Exercise brings on my fatigue.

2
c. I am easily fatigued........
2

3
4

5
6
d. Fatigue interferes with my physical functioning...1
2
e. Fatigue causes frequent problems for me.........
f. My fatigue prevents sustained physical functioning. .. 1
2

3

$\begin{array}{llll}4 & 5 & 6 & 7\end{array}$
g. Fatigue interferes with carrying out certain duties and responsibilities
most
disabling symptoms.
2

3

$\begin{array}{llll}4 & 5 & 6 & 7\end{array}$
Fatigue interferes with my work, family, or social life.
e................................. 1

$\begin{array}{lllllll}2 & 3 & 4 & 5 & 6 & 7\end{array}$


Please read each statement and circle a number $0,1,2$ or 3 which indicates how much the statement applied to you over the past week. There are no right or wrong answers. Do not spend too much time on any statement.

The rating scale is as follows:

0 Did not apply to me at all

1 Applied to me to some degree, or some of the time

2 Applied to me to a considerable degree, or a good part of time

3 Applied to me very much, or most of the time

1 I found it hard to wind down

2 I was aware of dryness of my mouth

3 I couldn't seem to experience any positive feeling at all

4 I experienced breathing difficulty (eg, excessively rapid breathing, breathlessness in the absence of physical exertion)

5 I found it difficult to work up the initiative to do things

$6 \quad$ I tended to over-react to situations

7 I experienced trembling (eg, in the hands)

8 I felt that I was using a lot of nervous energy

9 I was worried about situations in which I might panic and make a fool of myself

10 I felt that I had nothing to look forward to

11 I found myself getting agitated

12 I found it difficult to relax

13 I felt down-hearted and blue

14 I was intolerant of anything that kept me from getting on with what I was doing

15 I felt I was close to panic

16 I was unable to become enthusiastic about anything

17 I felt I wasn't worth much as a person

18 I felt that I was rather touchy

19 I was aware of the action of my heart in the absence of physical exertion (eg, sense of heart rate increase, heart missing a beat)

20 I felt scared without any good reason

21 I felt that life was meaningless

$\begin{array}{llll}0 & 1 & 2 & 3 \\ 0 & 1 & 2 & 3 \\ 0 & 1 & 2 & 3 \\ 0 & 1 & 2 & 3 \\ 0 & 1 & 2 & 3 \\ 0 & 1 & 2 & 3 \\ 0 & 1 & 2 & 3 \\ 0 & 1 & 2 & 3 \\ 0 & 1 & 2 & 3 \\ 0 & 1 & 2 & 3 \\ 0 & 1 & 2 & 3 \\ 0 & 1 & 2 & 3 \\ 0 & 1 & 2 & 3 \\ 0 & 1 & 2 & 3 \\ 0 & 1 & 2 & 3 \\ 0 & 1 & 2 & 3 \\ 0 & 1 & 2 & 3 \\ 0 & 1 & 2 & 3 \\ 0 & 1 & 2 & 3 \\ 0 & 1 & 2 & 3 \\ 0 & 1 & 2 & 3\end{array}$




\section{CESD-R}

Instructions: Please click the box which best describes how often you felt or behaved this way DURING THE PAST WEEK. The rating scale is as follows:

\begin{tabular}{|c|c|c|c|c|c|c|}
\hline 0 & 1 & \multicolumn{2}{|l|}{2} & \multicolumn{3}{|c|}{3} \\
\hline $\begin{array}{l}\text { Rarely or none of the } \\
\text { time; (less than } 1 \text { day a } \\
\text { week). }\end{array}$ & $\begin{array}{l}\text { Some or a little of the } \\
\text { time; ( } 1 \text { to } 2 \text { days a } \\
\text { week). }\end{array}$ & $\begin{array}{c}\text { Occasionally or a } \\
\text { moderate amount of } \\
\text { time; ( } 3 \text { to } 4 \text { days a } \\
\text { week). }\end{array}$ & & \multicolumn{3}{|c|}{$\begin{array}{l}\text { Most or all of the time; }(5 \\
\text { to } 7 \text { days a week). }\end{array}$} \\
\hline \multicolumn{3}{|c|}{ 1. I was bothered by things that usually don't bother me. } & 0 & $\mathbf{1}$ & 2 & 3 \\
\hline \multicolumn{3}{|c|}{ 2. I did not feel like eating; my appetite was poor. } & 0 & $\mathbf{1}$ & 2 & 3 \\
\hline \multicolumn{3}{|c|}{$\begin{array}{l}\text { 3. I felt that I could not shake off the blues even with help from my family or } \\
\text { friends. }\end{array}$} & $\mathbf{0}$ & 1 & 2 & 3 \\
\hline \multicolumn{3}{|c|}{ I felt that I was just as good as other people. } & 0 & $\mathbf{1}$ & 2 & 3 \\
\hline \multicolumn{3}{|c|}{ 5. I had trouble keeping my mind on what I was doing. } & 0 & 1 & 2 & 3 \\
\hline \multicolumn{3}{|l|}{ 6. I felt depressed. } & 0 & 1 & 2 & 3 \\
\hline \multicolumn{3}{|c|}{ 7. I felt that everything I did was an effort. } & 0 & 1 & 2 & 3 \\
\hline \multicolumn{3}{|c|}{ 8. I felt hopeful about the future. } & $\mathbf{0}$ & $\mathbf{1}$ & 2 & 3 \\
\hline \multicolumn{3}{|c|}{ 9. I thought my life had been a failure. } & $\mathbf{0}$ & 1 & 2 & 3 \\
\hline \multicolumn{3}{|l|}{ 10. I felt fearful. } & 0 & 1 & 2 & 3 \\
\hline \multicolumn{3}{|l|}{ 11. My sleep was restless. } & 0 & $\mathbf{1}$ & 2 & 3 \\
\hline \multicolumn{3}{|l|}{ 12. I was happy. } & $\mathbf{0}$ & $\mathbf{1}$ & 2 & 3 \\
\hline \multicolumn{3}{|l|}{ 13. I talked less than usual. } & 0 & 1 & 2 & 3 \\
\hline \multicolumn{3}{|l|}{ 14. I felt lonely. } & 0 & $\mathbf{1}$ & 2 & 3 \\
\hline \multicolumn{3}{|l|}{ 15. People were unfriendly. } & $\mathbf{0}$ & $\mathbf{1}$ & 2 & 3 \\
\hline \multicolumn{3}{|l|}{ 16. I enjoyed life. } & 0 & $\mathbf{1}$ & 2 & 3 \\
\hline \multicolumn{3}{|l|}{ 17. I had crying spells. } & $\mathbf{0}$ & 1 & 2 & 3 \\
\hline \multicolumn{3}{|l|}{ 18. I felt sad. } & $\mathbf{0}$ & $\mathbf{1}$ & 2 & 3 \\
\hline \multicolumn{3}{|c|}{ 19. I felt that people disliked me. } & $\mathbf{0}$ & 1 & 2 & 3 \\
\hline \multicolumn{3}{|l|}{ 20. I could not get going. } & 0 & 1 & 2 & 3 \\
\hline
\end{tabular}




\section{References}

Allavena, C., Guimard, T., Billaud, E., De la Tullaye, S., Reliquet, V., Pineau, S., . . Raffi, F. (2016). Prevalence and risk factors of sleep disturbance in a large HIV-infected adult population. AIDS and Behavior, 20(2), 339-344.

doi:http://dx.doi.org.ezproxy.lib.ryerson.ca/10.1007/s10461-015-1160-5

American Academy of Sleep Medicine (2005). International classification of sleep disorders:

Diagnostic and coding manual (2 $2^{\text {nd }}$ ed.). Westchester, NY: American Academy of Sleep Medicine. doi:10.1007/s13311-012-0145-6

American Psychiatric Association. (1994). Diagnostic and statistical manual of mental disorders ( $4^{\text {th }}$ ed.). Washington, DC: Author.

American Psychiatric Association. (2000). Diagnostic and statistical manual of mental disorders ( $4^{\text {th }}$ ed., text rev.). Washington, DC: Author.

American Psychiatric Association. (2013). Diagnostic and statistical manual of mental disorders (5 ${ }^{\text {th }}$ ed.). Washington, DC: Author. https://doi.org/10.1176/appi.books.9780890425596

Antony, M. M., Bieling, P. J., Cox, B. J., Enns, M. W., \& Swinson, R. P. (1998). Psychometric properties of the 42-item and 21-item versions of the Depression Anxiety Stress Scales in clinical groups and a community sample. Psychological Assessment, 10(2), 176-181. doi:http://dx.doi.org/10.1037/1040-3590.10.2.176

Arpinelli, F., Visona, G., Bruno, R., De Carli, G., \& Apolone, G. (2000). Health-related quality of life in asymptomatic patients with HIV. Evaluation of the SF-36 health survey in Italian patients. Pharmacoeconomics, 18(1), 63-72. https://doi.org/10.2165/00019053-20001801000007 
Asante, K. O. (2012). Social support and the psychological wellbeing of people living with HIV/AIDS in Ghana. African Journal of Psychiatry, 15(5), 340-345.

Babor, T. F., Higgins-Biddle, J. C., Saunders, J. B., \& Monteiro, M. G. (2001). AUDIT: The Alcohol Use Disorders Identification Test guidelines for use in primary care: World Health Organization. Geneva: Switzerland.

Battaglioli-DeNero, A. M. (2007). Strategies for improving patient adherence to therapy and long-term patient outcomes. Journal of the Association of Nurses in AIDS Care, 18(1S), S17-S22. https://doi.org/10.1016/j.jana.2006.11.020

Beck, A.T., Steer, R.A., \& Brown, G.K. (1996). Manual for the Beck Depression Inventory-II. San Antonio, TX: Psychological Corporation. https://doi.org/10.1037/t00742-000

Berman, A. H., Bergman, H., Palmstierna, T., \& Schlyter, F. (2005). Evaluation of the Drug Use Disorders Identification Test (DUDIT) in criminal justice and detoxification settings and in a Swedish population sample. European Addiction Research, 11(1), 22-31. doi:http://dx.doi.org/10.1159/000081413

Blashill, A. J., Safren, S. A., Wilhelm, S., Jampel, J., Taylor, S. W., O'Cleirigh, C., \& Mayer, K. H. (2017). Cognitive behavioral therapy for body image and self-care (CBT-BISC) in sexual minority men living with HIV: A randomized controlled trial. Health Psychology, 36(10), 937-946. doi: 10.1037/hea0000505

Bloom, M., \& Fisher, J. (1982). Evaluating practice: Guidelines for the accountable professional. Englewood Cliffs, NJ: Prentice Hall.

Boender, T. S., Sigaloff, K. C., McMahon, J. H., Kiertiburanakul, S., Jordan, M. R., Barcarolo, J., ... Bertagnolio, S. (2015). Long-term virological outcomes of first-line antiretroviral 
therapy for HIV-1 in low- and middle-income countries: A systematic review and metaanalysis. Clinical Infectious Diseases, 61(9), 1453-1461. https://doi.org/10.1093/cid/civ556

Bogart, L. M., Dale, S. K., Daffin, G. K., Patel, K. N., Klein, D. J., Mayer, K. H., \& Pantalone, D. W. (2018). Pilot intervention for discrimination-related coping among HIV-positive black sexual minority men. Cultural Diversity and Ethnic Minority Psychology, 24(4), 541-551. doi:http://dx.doi.org.ezproxy.lib.ryerson.ca/10.1037/cdp0000205

Bootzin, R. (1972). Stimulus control treatment for insomnia. Proceedings of the 80th Annual Convention of the American Psychological Association, Honolulu, HI, 7, 395-396.

Borkovec, T., Nau, S. D. (1972). Credibility of analogue therapy rationales. Journal of Behavior Therapy and Experimental Psychiatry, 3, 247-260. https://doi.org/10.1016/00057916(72)90045-6

Born, J., Lange, T., Hansen, K., Molle, M., \& Fehm, H. L. (1997). Effects of sleep and circadian rhythm on human circulating immune cells. Journal of Immunology, 158, 4454-4464.

Braun, V., \& Clarke, V. (2006). Using thematic analysis in psychology. Qualitative Research in Psychology, 3, 77-101. doi: 10.1191/1478088706qp063oa

British Columbia Centre for Excellence in HIV/AIDS. (2018). Therapeutic guidelines for antiretroviral (ARV) treatment of adult HIV infection. Retrieved from http://cfenet.ubc.ca/sites/default/files/uploads/Guidelines/Therapeutic-Guidelines-forAntiretroviral-ARV-Treatment-of-Adult-HIV-Infection-[07-JUN-2018].pdf

Broadbent, E., Petrie, K. J., Main, J., \& Weinman, J. (2006). The Brief Illness Perception Questionnaire. Journal of Psychosomatic Research, 60(6), 631-637. doi:http://dx.doi.org/10.1016/j.jpsychores.2005.10.020 
Broomfield, N. M., \& Espie, C. A. (2005). Towards a valid, reliable measure of sleep effort. Journal of Sleep Research, 14(4), 401-407. doi:http://dx.doi.org/10.1111/j.13652869.2005.00481.x

Brown, S., Mitler, M., \& Atkinson, H. (1991). Correlation of subjective sleep complaints, absolute T-4 cell number and anxiety in HIV illness. Sleep Research, 20, 363.

Budhiraja, R., Roth, T., Hudgel, D. W., Budhiraja, P., \& Drake, C. L. (2011). Prevalence and polysomnographic correlates of insomnia comorbid with medical disorders. Sleep, 34(7), 859-867. https://doi.org/10.5665/SLEEP.1114

Busk, P. L., \& Marascuilo, L. A. (2015) Statistical analysis in single-case research: Issues, procedures, and recommendations, with applications to multiple behaviors. In T. R. Kratochwill \& J. R. Levin (Eds.), Single-case research design and analysis: New directions for psychology and education (pp. 159-186). New York, NY: Routledge.

Burchell, A. N., Gardner, S., Light, L., Ellis, B. M., Antoniou, T., Bacon, J., . . Rourke, S. B. (2015). Engagement in HIV care among persons enrolled in a clinical HIV cohort in Ontario, Canada, 2001-2011. Journal of Acquired Immune Deficiency Syndromes, 70(1), e10-e19. https://doi.org/10.1097/QAI.0000000000000690

Burkhalter, J. E., Cahill, S., Shuk, E., Guidry, J., Corner, G., Berk, A., . . Lubetkin, E. I. (2013). At the intersection of HIV/AIDS and cancer: A qualitative needs assessment of community-based HIV/AIDS service organizations. Health Education \& Behavior, 40(4), 493-503. https://doi.org/10.1177/1090198112459049

Buysse, D. J., Reynolds, C. F. III., Monk, T. H., Berman, S. R., \& Kupfer, D. J. (1989). The Pittsburgh Sleep Quality Index: A new instrument for psychiatric practice and research. Psychiatry Research, 28, 193-213. https://doi.org/10.1016/0165-1781(89)90047-4 
Carey, T. J., Moul, D. E., Pilkonis, P., Germain, A., \& Buysse, D. J. (2005). Focusing on the experience of insomnia. Behavioral Sleep Medicine, 3(2), 73-86.

doi:http://dx.doi.org/10.1207/s15402010bsm0302_2

Carney, C. E., Buysse, D. J., Ancoli-Israel, S., Edinger, J. D., Krystal, A. D., Lichstein, K. L., \& Morin, C. M. (2012). The Consensus Sleep Diary: Standardizing prospective sleep selfmonitoring. Sleep, 35(2), 287-302. https://doi.org/10.5665/sleep.1642

Carney, C. E., Edinger, J. D., Kuchibhatla, M., Lachowski, A. M., Bogouslavsky, O., Krystal, A. D., \& Shapiro, C. M. (2017). Cognitive behavioral insomnia therapy for those with insomnia and depression: A randomized controlled clinical trial. Sleep, 40(4), 1-13. https://doi.org/10.1093/sleep/zsx019

Carney, C. E., Edinger, J. D., Morin, C. M., Manber, R., Rybarczyk, B., Stepanski, E. J., . . . Lack, L. (2010). Examining maladaptive beliefs about sleep across insomnia patient groups. Journal of Psychosomatic Research, 68(1), 57-65. https://doi.org/10.1016/j.jpsychores.2009.08.007

Carney, C. E., Edinger, J. D., Olsen, M. K., Stechuchak, K. M., Krystal, A. D., \& Wyatt, J. K. (2008). Inter-rater reliability for insomnia diagnoses derived from the Duke Structured Interview for Sleep Disorders. Sleep, 31(Supp1.), A250. https://doi.org/10.1093/sleep/31.5.599

Carney, C. E., \& Manber, R. (2009). Quiet your mind and get to sleep. Oakland, CA: New Harbinger.

Carrieri, P., Spire, B., Duran, S., Katlama, C., Peyramond, D., Francois, C., ... APROCO Study Group. (2003). Health-related quality of life after 1 year of highly active antiretroviral 
therapy. Journal of Acquired Immune Deficiency Syndromes, 32(1), 38-47.

https://doi.org/10.1097/00126334-200301010-00006

Chesney, M. (2003). Adherence to HAART regimens. AIDS Patient Care and STDs, 17, 169177. https://doi.org/10.1089/108729103321619773

Cheung, J. M. Y., Bartlett, D. J., Armour, C. L., Glozier, N., \& Saini, B. (2014). Insomnia patients' help-seeking experiences. Behavioral Sleep Medicine, 12(2), 106-122. doi:http://dx.doi.org/10.1080/15402002.2013.764529

Choi, S. K. Y., Boyle, E., Burchell, A.N., Gardner, S., Collins, E., Grootendorst, P., ... OHTN Cohort Study Group. (2015). Validation of six short and ultra-short screening instruments for depression for people living with HIV in Ontario: Results from the Ontario HIV Treatment Network Cohort Study. PLoS ONE 10(11): e0142706. doi:10.1371/journal.pone.0142706

Choi, S. K. Y., Boyle, E., Cairney, J., Collins, E. J., Gardner, S., Bacon, J., \& Rourke, S. B. (2016). Prevalence, recurrence, and incidence of current depressive symptoms among people living with HIV in Ontario, Canada: Results from the Ontario HIV Treatment Network Cohort Study. PLoS ONE, 11(11): e0165816. doi: 10.1371/journal.pone.0165816

Choi, S. K. Y., Boyle, E., Cairney, J., Gardner, Collins, E. J., S., Bacon, J., ... OHTN Cohort Study Group. (2016). Adequacy of mental health services for HIV-positive patients with depression: Ontario HIV Treatment Network Cohort Study. PLoS ONE, 11(6): e0156652. doi: 10.1371/journal.pone.0156652

Chung, F., Subramanyam, R., Liao, P., Sasaki, E., Shapiro, C., \& Sun, Y. (2012). High STOPBang score indicates a high probability of obstructive sleep apnoea. British Journal of Anaesthesia, 108(5), 768-775. https://doi.org/10.1093/bja/aes022 
Chung, F., Yegneswaran, B., Liao, P., Chung, S. A., Vairavanathan, S., Islam, S., \& Shapiro, C. (2008). STOP questionnaire: A tool to screen patients for obstructive sleep apnea. Anesthesiology, 108(5), 812-821. https://doi.org/10.1097/ALN.0b013e31816d83e4

Chung, K., Yeung, W., Ho, F. Y., Yung, K., Yu, Y., \& Kwok, C. (2015). Cross-cultural and comparative epidemiology of insomnia: The Diagnostic and Statistical Manual (DSM), International Classification of Diseases (ICD) and International Classification of Sleep Disorders (ICSD). Sleep Medicine, 16(4), 477-482. doi:http://dx.doi.org/10.1016/j.sleep.2014.10.018

Cohen, S., Doyle, W. J., Alper, C. M., Janicki-Deverts, D., \& Turner, R. B. (2009). Sleep habits and susceptibility to the common cold. Archives of Internal Medicine, 169(1), 62-67. doi:10.1001/archinternmed.2008.505

Cohen. F. L., Ferrans, C., E., Vizgirda, V., Kunkle, V., \& Cloninger, L. (1996). Sleep in men and women infected with human immunodeficiency virus. Holistic Nursing Practice, 10, 3343. https://doi.org/10.1097/00004650-199607000-00007

Corless, I. B., Voss, J. G., Nicholas, P. K., Bunch, E. H., Bain, C. A., Coleman, C., ... Valencia, C. P. (2008). Fatigue in HIV/AIDS patients with comorbidities. Applied Nursing Research, 21, 116-122. doi:10.1016/j.apnr.2006.11.001

Creswell, J. W. (2014). Research design: Qualitative, quantitative, and mixed methods approaches $\left(4^{\text {th }}\right.$ ed. $)$. Thousand Oaks, CA: Sage.

Cruess, D. G., Antoni, M. H., Gonzalez, J., Fletcher, M. A., Klimas, N., Duran, R., ... Schneiderman, N. (2003). Sleep disturbance mediates the association between psychological distress and immune status among HIV-positive men and women on 
combination antiretroviral therapy. Journal of Psychosomatic Research, 54(3), 185-189. https://doi.org/10.1016/S0022-3999(02)00501-9

Crum-Cianflone, N. F., Roediger, M. P., Moore, D. J., Hale, B., Weintrob, A., Ganesan, A., ... \& Letendre, S. (2012). Prevalence and factors associated with disturbances among earlytreated HIV-infected persons. Clinical Infectious Diseases, 54(10), 1485-1494. doi:10.1093/cid/cis192

Currie, S. R., Wilson, K. G., \& Curran, D. (2002). Clinical significance and predictors of treatment response to cognitive-behavior therapy for insomnia secondary to chronic pain. Journal of Behavioral Medicine, 25(2), 135-153. doi:http://dx.doi.org/10.1023/A:1014832720903

Darko, D. F., McCutchan, A., Kripke, D. F., Gillin, J. C., \& Golshan, S. (1992). Fatigue, sleep disturbance, disability, and indices of progression of HIV infection. The American Journal of Psychiatry, 149(4), 514-520. https://doi.org/10.1176/ajp.149.4.514

Davidson, J. R., Feldman-Stewart, D., Brennenstuhl, S., \& Ram, S. (2007). How to provide insomnia interventions to people with cancer; Insights from patients. PsychoOncology, 16(11), 1028-1038. doi:http://dx.doi.org/10.1002/pon.1183

Davis, L., Evans, S., Fishman, B., Haley, A., \& Spielman, L. A. (2004). Predictors of attrition in HIV-positive subjects with peripheral neuropathic pain. AIDS Care, 16(3), 395-402. doi:http://dx.doi.org.ezproxy.lib.ryerson.ca/10.1080/09540120410001665394

Denscombe, M. (2008). Communities of practice: A research paradigm for the mixed methods approach. Journal of Mixed Methods Research, 2(3), 270-283.

https://doi.org/10.1177/1558689808316807 
Doerfler, R. E., \& Goodfellow, L. (2016). Brief exposure to cognitive behavioral therapy reduces side-effect symptoms in patients on antiretroviral therapy. Journal of the Association of Nurses in AIDS Care, 27(4), 455. doi:http://dx.doi.org.ezproxy.lib.ryerson.ca/10.1016/j.jana.2016.02.010

Dreher, H. M. (2003). The effect of caffeine reduction on sleep quality and well-being in persons with HIV. Journal of Psychosomatic Research, 54(3), 191-198. doi:http://dx.doi.org/10.1016/S0022-3999(02)00472-5

Edinger, J. D., \& Carney, C. E. (2008). Overcoming insomnia: A cognitive-behavioral therapy approach. Therapist guide. New York: Oxford University Press. https://doi.org/10.1093/med:psych/9780195365894.001.0001

Edinger, J. D., Hoelscher, T. J., Marsh, G. R., Lipper, S., \& Ionescue-Pioggia, M. (1992). A cognitive-behavioral therapy for sleep-maintenance insomnia in older adults. Psychology and Aging, 7, 282-289. doi:http://dx.doi.org/10.1037/0882-7974.7.2.282

Edinger, J. D., Wohlgemuth, W. K., Radtke, R. A., Coffman, C. J., \& Carney, C. E. (2007). Dose-response effects of cognitive-behavioral insomnia therapy; A randomized clinical trial. Sleep, 30, 203-212. https://doi.org/10.1093/sleep/30.2.203

Edinger, J. D., Wohlgemuth, W. K., Radtke, R. A., Marsh, G. R., \& Quillian, R. E. (2001). Cognitive behavioral therapy for treatment of chronic primary insomnia: A randomized controlled trial. Journal of the American Medical Association, 285(14), 1856-1864. doi:http://dx.doi.org/10.1001/jama.285.14.1856

Edinger, J. D., Wyatt, J. K., Olsen, M. K., Stechuchak, K. M., Carney, C. E., Chiang, A., ... \& Radtke, R. A. (2009). Reliability and validity of the Duke Structured Interview for Sleep 
Disorders for insomnia screening. Sleep, 32(suppl), A265.

https://doi.org/10.1093/sleep/32.4.499

Epstein, L. J., Strollo, P. J. J., Donegan, R. B., Delmar, J., Hendrix, C., \& Westbrook, P. R. (1995). Obstructive sleep apnea in patients with human immunodeficiency virus (HIV) disease. Sleep, 18, 368-376. https://doi.org/10.1093/sleep/18.5.368

Espie, C. A., Broomfield, N. M., MacMahon, K. M. A., Macphee, L. M., \& Taylor, L. M. (2006). The attention-intention-effort pathway in the development of psychophysiologic insomnia: A theoretical review. Sleep Medicine Reviews, 10(4), 215-245. https://doi.org/10.1016/j.smrv.2006.03.002

Espie, C. A., Kyle, S. D., Miller, C. B., Ong, J., Hames, P., \& Fleming, L. (2014). Attribution, cognition and psychopathology in persistent insomnia disorder: Outcome and mediation analysis from a randomized placebo-controlled trial of online cognitive behavioural therapy. Sleep Medicine, 15(8), 913-917. doi:http://dx.doi.org/10.1016/j.sleep.2014.03.001

Evans, B. C., Coon, D. W., \& Ume, E. (2011). Use of theoretical frameworks as a pragmatic guide for mixed methods studies: A methodological necessity? Journal of Mixed Methods Research, 5(4), 276-292. doi:http://dx.doi.org/10.1177/1558689811412972

Evans, D. L., Ten Have, T. R., Douglas, S. D., Gettes, D. R., Morrison, M., Chiappini, M. S., ... Pettito, J. M. (2002). Association of depression with viral load, CD8 T lymphocytes, and natural killer cells in women with HIV infection. The American Journal of Psychiatry, 159, 1752-1759. https://doi.org/10.1176/appi.ajp.159.10.1752

Fairholme, C. P., \& Manber, R. (2014). Safety behaviors and sleep effort predict sleep disturbance and fatigue in an outpatient sample with anxiety and depressive disorders. 
Journal of Psychosomatic Research, 76, 233-236.

doi:http://dx.doi.org/10.1016/j.jpsychores.2014.01.001

Fasula, A. M., Fogel, C. I., Gelaude, D., Carry, M., Gaiter, J., \& Parker, S. (2013). Project

Power: Adapting an evidence-based HIV/STI prevention intervention for incarcerated women. AIDS Education and Prevention, 25(3), 203-15.

doi:http://dx.doi.org/10.1521/aeap.2013.25.3.203

Feldman, B. J., Fredericksen, R. J., Crane, P. K., Safren, S. A., Mugavero, M. J., Willig, J. H., ,.. Crane, H. M. (2013). Evaluation of the single-item self-rating adherence scale for use in routine clinical care of people living with HIV. AIDS and Behavior, 17(1), 307-318. doi:http://dx.doi.org/10.1007/s10461-012-0326-7

Ferini-Strambi, L., Oldani, A., Tirloni, G., Zucconi, M., Castagna, A., Lazzarin, A., \& Smirne, S. (1995). Slow wave sleep and cyclic alternating pattern (CAP) in HIV-infected asymptomatic men. Sleep, 18(6), 446-450.

Fichten, C. S., Libman, E., Creti, L., Amsel, R., Sabourin, S., Brender, W., \& Bailes, S. (2001). Role of thoughts during nocturnal awake times in the insomnia experience of older adults. Cognitive Therapy and Research, 25, 665-692. https://doi.org/10.1023/A:1012963121729

First, M. B., Spitzer, R. L., Gibbon, M., \& Williams, J. B. W. (2002). Structured Clinical Interview for DSM-IV-TR Axis I Disorders, Research Version, Non-patient Edition (SCIDI/NP). New York: Biometrics Research, New York State Psychiatric Institute.

Fleming, L., Gillespie, S., \& Espie, C. A. (2010). The development and impact of insomnia on cancer survivors: A qualitative analysis. Psycho-Oncology, 19(9), 991-996. doi:http://dx.doi.org/10.1002/pon.1652 
Gallego, L., Barreiro, P., del Río, R., González de Requena, D., Rodríguez-Albariño, A., González-Lahoz, J., \& Soriano, V. (2004). Analyzing sleep abnormalities in HIV-infected patients treated with efavirenz. Clinical Infectious Diseases, 38, 430-432. https://doi.org/10.1086/380791

Gamaldo, C. E., Spira, A. P., Hock, R. S., Salas, R. E., McArthur, J. C., David, P. M., ... Smith, M. T. (2013). Sleep, function and HIV: A multi-method assessment. AIDS and Behavior, 17(8), 2808-2815. doi:http://dx.doi.org/10.1007/s10461-012-0401-0

Garland, S. N., Johnson, J. A., Savard, J., Gehrman, P., Perlis, M., Carlson, L., \& Campbell, T. (2014). Sleeping well with cancer: A systematic review of cognitive behavioral therapy for insomnia in cancer patients. Neuropsychiatric Disease and Treatment, 10, 1113-1124. https://doi.org/10.2147/NDT.S47790

Gay, C., Portillo, C. J., Kelly, R., Coggins, T., Davis, H., Aouizerat, B. E., ... Lee, K. A. (2011). Self-reported medication adherence and symptom experience in adults with HIV. Journal of the Association of Nurses in AIDS Care, 22(4), 257-268. https://doi.org/10.1016/j.jana.2010.11.004

Gay, C. L., Kottorp, A., Lerdal, A., \& Lee, K. A. (2016). Psychometric limitations of the Center for Epidemiologic Studies-Depression scale for assessing depressive symptoms among adults with HIV/AIDS: A Rasch analysis. Depression Research and Treatment, 2016, 111. https://doi.org/10.1155/2016/2824595

Gay, C. L., Zak, R. S., Lerdal, A., Pullinger, C. R., Aouizerat, B. E., \& Lee, K. A. (2015). Cytokine polymorphisms and plasma levels are associated with sleep onset insomnia in adults living with HIV/AIDS. Brain, Behavior, and Immunity, 47, 58-65. doi:http://dx.doi.org/10.1016/j.bbi.2014.11.018 
Germain, A., Shear, M. K., Hall, M., \& Buysse, D. J. (2007). Effects of a brief behavioral treatment for PTSD-related sleep disturbances: A pilot study. Behaviour Research and Therapy, 45(3), 627-632. doi:http://dx.doi.org/10.1016/j.brat.2006.04.009

Goforth, H. W., Cohen, M. A., \& Murrough, J. (2008). Mood Disorders. In: M. A. Cohen, J. M. Gorman, Eds. Comprehensive textbook of AIDS psychiatry. New York: Oxford.

Goldstein, L. H., Mellers, J. D. C., Landau, S., Stone, J., Carson, A., Medford, N., . . Chalder, T. (2015). Cognitive behavioural therapy vs standardised medical care for adults with dissociative non-epileptic seizures (CODES): A multicentre randomised controlled trial protocol. BMC Neurology, 15(98). 1-13. https://doi.org/10.1186/s12883-015-0350-0

Goswami, U., Baker, J. V., Wang, Q., Khalil, W., \& Kunisaki, K. M. (2015). Sleep apnea symptoms as a predictor of fatigue in an urban HIV clinic. AIDS Patient Care and STDs, 29(11), 591-596. doi:http://dx.doi.org.ezproxy.lib.ryerson.ca/10.1089/apc.2015.0079

Gottman, J. M., \& Leiblum, S. R. (1974). How to do psychotherapy and how to evaluate it. New York: Holt, Rinehart, \& Winston.

Grant, K., McMeekin, E., Jamieson, R., Fairfull, A., Miller, C., \& White, J. (2012). Individual therapy attrition rates in a low-intensity service: A comparison of cognitive behavioural and person-centred therapies and the impact of deprivation. Behavioural and Cognitive Psychotherapy, 40(2), 245-249. doi:http://dx.doi.org.ezproxy.lib.ryerson.ca/10.1017/S1352465811000476

Green, A., Hicks, J., \& Wilson, S. (2008). The experience of poor sleep and its consequences: A qualitative study involving people referred for cognitive-behavioural management of chronic insomnia. The British Journal of Occupational Therapy, 71(5), 196-204. doi:http://dx.doi.org/10.1177/030802260807100506 
Hacker, K. (2013). Community-based participatory research. Thousand Oaks, CA: Sage.

Haddad, N., Li, J. S., Totten, S., \& McGuire, M. (2018). HIV in Canada—surveillance report, 2017. Canadian Communicable Disease Report, 44(12), 324-332. https://doi.org/10.14745/ccdr.v44i12a03

Harden, R. N., Weinland, S. R., Remble, T. A., Houle, T. T., Colio, S., Steedman, S., \& Kee, W. G. (2005). Medication Quantification Scale Version III: Update in medication classes and revised detriment weights by survey of American Pain Society physicians. The Journal of Pain, 6(6), 364-371. doi:http://dx.doi.org/10.1016/j.jpain.2005.01.350

Hart, T. A., Willis, A. C., Simpson, S. H., Julien, R. E., Hoe, D., Leahy, B., . . Adam, B. D. (2016). Gay poz sex: A sexual health promotion intervention for HIV-positive gay and bisexual men. Cognitive and Behavioral Practice, 23(4), 517-529. doi:http://dx.doi.org.ezproxy.lib.ryerson.ca/10.1016/j.cbpra.2015.11.002

Harvey, A. G. (2002). A cognitive model of insomnia. Behavior Research and Therapy, 40(8), 869-893. https://doi.org/10.1016/S0005-7967(01)00061-4

Harvey, A. G., Soehner, A., Lombrozo, T., Bélanger, L., Rifkin, J., \& Morin, C. M. (2013). 'Folk theories' about the causes of insomnia. Cognitive Therapy and Research, 37(5), 1048-1057. doi:http://dx.doi.org/10.1007/s10608-013-9543-2

Hautzinger, M., Keller, F., \& Kühner, C. (2009). BDI-II. Beck-Depressions-Inventar. Revision. 2. Auflage. Frankfurt: Pearson Assessment.

Hayes, A. F. (2013). Introduction to mediation, moderation, and conditional process analysis. New York: Guilford. 
Henry, D., Rosenthal, L., Dedrick, D., \& Taylor, D. (2013). Understanding patient responses to insomnia. Behavioral Sleep Medicine, 11(1), 40-55. doi:http://dx.doi.org/10.1080/15402002.2011.620671

Henry, J. D., \& Crawford, J. R. (2005). The short-form version of the Depression Anxiety Stress Scales (DASS-21): Construct validity and normative data in a large non-clinical sample. British Journal of Clinical Psychology, 44(2), 227-239. doi:http://dx.doi.org/10.1348/014466505X29657

Henry, S. K., Grant, M. M., \& Cropsey, K. L. (2018). Determining the optimal clinical cutoff on the CES-D for depression in a community corrections sample. Journal of Affective Disorders, 234, 270-275. https://doi.org/10.1016/j.jad.2018.02.071

Hoelscher, T. J., \& Edinger, J. D. (1988). Treatment of sleep-maintenance insomnia in older adults: Sleep period reduction, sleep education, and modified stimulus control. Psychology and Aging, 3, 258-263. doi:http://dx.doi.org/10.1037/0882-7974.3.3.258

Horberg, M. A., Silverberg, M. J., Hurley, L. B., Towner, W. J., Klein, D. B., Bersoff-Matcha, S., ... Kovach, D. A. (2008). Effects of depression and selective serotonin reuptake inhibitor use on adherence to highly active antiretroviral therapy and on clinical outcomes in HIV-infected patients. Journal of Acquired Immune Deficiency Syndromes, 47(3), 384390. https://doi.org/10.1097/QAI.0b013e318160d53e

Hsiung, P., Fang, C., Chang, Y., Chen, M., \& Wang, J. (2005). Comparison of WHOQOL-BREF and SF-36 in patients with HIV infection. Quality of Life Research, 14(1), 141-150. doi:http://dx.doi.org/10.1007/s11136-004-6252-z 
Hudson, A. L., Portillo, C. J., \& Lee, K. A. (2008). Sleep disturbances in women with HIV or AIDS: Efficacy of a tailored sleep promotion intervention. Nursing Research, 57(5), 360366. doi:http://dx.doi.org/10.1097/01.NNR.0000313501.84604.2c

Ickovics, J. R., Hamburger, M. E., Vlahov, D., Schoenbaum, E. E., Schuman, P., Boland, R. J., \& Moore, J. (2001). Mortality, CD4 cell count decline, and depressive symptoms among HIV-seropositive women: Longitudinal analyses from the HIV epidemiology research study. Journal of the American Medical Association, 285(11), 1466-1474.

https://doi.org/10.1001/jama.285.11.1466

Ironson, G., O'Cleirigh, C., Fletcher, M. A., Laurenceau, J. P., Balbin, E., Klimas, N., ... Solomon, G. (2005). Psychosocial factors predict CD4 and viral load change in men and women with human immunodeficiency virus in the era of highly active antiretroviral treatment. Psychosomatic Medicine, 67(6), 1013-1021. https://doi.org/10.1097/01.psy.0000188569.58998.c8

Irwin, M. R. (2015). Why sleep is important for health: A psychoneuroimmunology perspective. Annual Review of Psychology, 66, 143-172. doi:http://dx.doi.org/10.1146/annurev-psych-010213-115205

Irwin, M., Clark, C., Kennedy, B., Gillin, J. C., \& Ziegler, M. (2003). Nocturnal catecholamines and immune function in insomniacs, depressed patients, and control subjects. Brain, Behavior, and Immunity, 17(5), 365-372. doi:http://dx.doi.org/10.1016/S08891591(03)00031-X

Irwin, M., McClintick, J., Costlow, C., Fortner, M., White, J., \& Gillin, J. C. (1996). Partial night sleep deprivation reduces natural killer and cellular immune response in humans. The FASEB Journal, 10(5), 643-653. https://doi.org/10.1096/fasebj.10.5.8621064 
Jacobs, G. D., Pace-Schott, E. F., Stickgold, R., \& Otto, M. W. (2004). Cognitive behavior therapy and pharmacotherapy for insomnia: A randomized controlled trial and direct comparison. Archives of Internal Medicine, 164(17), 1888-1896. doi:10.1001/archinte.164.17.1888

Jansson-Fröjmark, M., \& Linton, S. J. (2008). The course of insomnia over one year: A longitudinal study in the general population in Sweden. Sleep, 31(6), 881-886. https://doi.org/10.1093/sleep/31.6.881

Jansson-Fröjmark, M., \& Norell-Clarke, A. (2012). Psychometric properties of the Pre-Sleep Arousal Scale in a large community sample. Journal of Psychosomatic Research, 72(2), 103-110. doi:http://dx.doi.org/10.1016/j.jpsychores.2011.10.005

Jean-Louis, G., Weber, K. M., Aouizerat, B. E., Levine, A. M., Maki, P. M., Liu, C., .. . Wilson, T. E. (2012). Insomnia symptoms and HIV infection among participants in the Women's Interagency HIV Study. Sleep, 35(1), 131-137. https://doi.org/10.5665/sleep.1602

Johansson, S., Kottorp, A., Lee, K. A., Gay, C. L., \& Lerdal, A. (2014). Can the Fatigue Severity Scale 7-item version be used across different patient populations as a generic fatigue measure-A comparative study using a Rasch model approach. Health and Quality of Life Outcomes, 12(24), 1-9. https://doi.org/10.1186/1477-7525-12-24

Johns, M. W. (1991). A new method for measuring daytime sleepiness: The Epworth Sleepiness Scale. Sleep, 14, 540-545. https://doi.org/10.1093/sleep/14.6.540

Johns, M. W. (1992). Reliability and factor analysis of the Epworth Sleepiness Scale. Sleep, 15(4), 376-381. https://doi.org/10.1093/sleep/15.4.376

Jungquist, C. R., O’Brien, C., Matteson-Rusby, S., Smith, M. T., Pigeon, W. R., Xia, Y., .. . Perlis, M. L. (2010). The efficacy of cognitive-behavioral therapy for insomnia in patients 
with chronic pain. Sleep Medicine, 11(3), 302-309.

doi:http://dx.doi.org/10.1016/j.sleep.2009.05.018

Jungquist, C. R., Tra, Y., Smith, M. T., Pigeon, W. R., Matteson-Rusby, S., Xia, Y., \& Perlis, M. L. (2012). The durability of cognitive behavioral therapy for insomnia in patients with chronic pain. Sleep Disorders, 2012, 1-8. doi:10.1155/2012/679648

Justice, A. C., Holmes, W., Gifford, A. L., Rabeneck, L., Zackin, R., Sinclair, G., .. Wu, A. W. (2001). Development and validation of a self-completed HIV symptom index. Journal of Clinical Epidemiology, 54, S77-S90. https://doi.org/10.1016/S0895-4356(01)00449-8

Kalichman, S. C., Rompa, D., \& Cage, M. (2000). Distinguishing between overlapping somatic symptoms of depression and HIV disease in people living with HIV-AIDS. Journal of Nervous and Mental Disease, 188(10), 662-670. https://doi.org/10.1097/00005053200010000-00004

Karlin, B. E., Trockel, M., Spira, A. P., Taylor, C. B., \& Manber, R. (2015). National evaluation of the effectiveness of cognitive behavioral therapy for insomnia among older versus younger veterans. International Journal of Geriatric Psychiatry, 30, 308-315. https://doi.org/10.1002/gps.4143

Kazdin, A. E. (1982). Single-case research designs: Methods for clinical and applied settings. New York, NY: Oxford University Press.

Kenedi, C. A., \& Goforth, H. W. (2011). A systematic review of the psychiatric side-effects of efavirenz. AIDS and Behavior, 15(8), 1803-1818. doi:http://dx.doi.org/10.1007/s10461011-9939-5

Khalil, E., Callaghan, P., Carter, T., \& Morres, I. (2012). Pragmatic randomised controlled trial of an exercise programme to improve wellbeing outcomes in women with depression: 
Findings from the qualitative component. Psychology, 3(11), 979-

986. ttps://doi.org/10.4236/psych.2012.311147

Kitahata, M. M., Reed, S. D., Dillingham, P. W., Van Rompaey, S. E., Young, A. A., Harrington, R. D., \& Holmes, K. K. (2004). Pharmacy-based assessment of adherence to HAART predicts virologic and immunologic treatment response and clinical progression to AIDS and death. International Journal of STD \& AIDS, 15(12), 803-810. https://doi.org/10.1258/0956462042563666

Klinkenberg, W. D. \& Sacks, S. (2004). Mental disorders and drug abuse in persons living with HIV/AIDS. AIDS Care, 16, S22-S42. https://doi.org/10.1080/09540120412331315303

Knobel, H., Alonso, J., Casado, J. L., Collazos, J., Gonzalez, J., Ruiz, I., ... Ocampo, A. (2002). Validation of a simplified medication adherence questionnaire in a large cohort of HIVinfected patients: The GEEMA study. AIDS, 16(4), 605-613. https://doi.org/10.1097/00002030-200203080-00012

Koffel, E. A., Koffel, J. B., \& Gehrman, P. R. (2015). A meta-analysis of group cognitive behavioral therapy for insomnia. Sleep Medicine Reviews, 19, 6-16. https://doi.org/10.1016/j.smrv.2014.05.001

Kronfol, Z., Nair, M., Zhang, Q., Hill, E. E., \& Brown, M. B. (1997). Circadian immune measures in healthy volunteers: Relationship to hypothalamic-pituitary-adrenal axis hormones and sympathetic neurotransmitters. Psychosomatic Medicine, 59(1), 4250. https://doi.org/10.1097/00006842-199701000-00006

Krupp, L. B., LaRocca, N. G., Muir-Nash, J., \& Steinberg, A. D. (1989). The Fatigue Severity Scale. Application to patients with multiple sclerosis and systemic lupus erythematosus. 
Archives of Neurology, 46, 1121-1123.

https://doi.org/10.1001/archneur.1989.00520460115022

Kyle, S. D., Espie, C. A., \& Morgan, K. (2010). “... Not just a minor thing, it is something major, which stops you from functioning daily": Quality of life and daytime functioning in insomnia. Behavioral Sleep Medicine, 8(3), 123-140.

doi:http://dx.doi.org/10.1080/15402002.2010.487450

Kyle, S. D., Morgan, K., Spiegelhalder, K., \& Espie, C. A. (2011). No pain, no gain: An exploratory within-subjects mixed-methods evaluation of the patient experience of sleep restriction therapy (SRT) for insomnia. Sleep Medicine, 12(8), 735-747. doi:http://dx.doi.org/10.1016/j.sleep.2011.03.016

Lacks, P. (1987). Psychology practitioner guidebooks. New York: Pergamon Press.

Lancee, J., van den Bout, J., van Straten, A., \& Spoormaker, V. I. (2013). Baseline depression levels do not affect efficacy of cognitive-behavioral self-help treatment for insomnia. Depression and Anxiety, 30(2), 149-156. doi:http://dx.doi.org/10.1002/da.22004

Landstra, J. M. B., Ciarrochi, J., Deane, F. P., \& Hillman, R. J. (2013). Identifying and describing feelings and psychological flexibility predict mental health in men with HIV. British Journal of Health Psychology, 18(4), 844-857. doi:http://dx.doi.org/10.1111/bjhp.12026

Lee, K. A., Gay, C., Portillo, C. J., Coggins, T., Davis, H., Pullinger, C. R., \& Aouizerat, B. E. (2012). Types of sleep problems in adults living with HIV/AIDS. Journal of Clinical Sleep Medicine, 8(1), 67-75. https://doi.org/10.5664/jcsm.1666 
Lee, K. A., Portillo, C. J., \& Miramontes, H. (2001). The influence of sleep and activity patterns on fatigue in women with HIV/AIDS. Journal of the Association of Nurses in AIDS Care, 12,Suppl, 19-27. https://doi.org/10.1016/S1055-3290(06)60154-4

Lerdal, A., Kottorp, A., Gay, C., Aouizerat, B. E., Portillo, C. J., \& Lee, K. A. (2011). A 7-item version of the fatigue severity scale has better psychometric properties among HIVinfected adults: an application of a Rasch model. Quality of Life Research, 20, 1447-1456. https://doi.org/10.1007/s11136-011-9877-8

Li, L., Wu, C., Gan, Y., Qu, X., \& Lu, Z. (2016). Insomnia and the risk of depression: a metaanalysis of prospective cohort studies. BMC Psychiatry, 16, 375-390. https://doi.org/10.1186/s12888-016-1075-3

Liu, Y., Canada, K., Shi, K., \& Corrigan, P. (2012). HIV-related stigma acting as predictors of unemployment among people living with HIV/AIDS. AIDS Care, 24(1), 129-135. https://doi.org/10.1080/09540121.2011.596512

Lobbestael, J., Leurgans, M., \& Arntz, A. (2011). Inter-rater reliability of the Structured Clinical Interview for DSM-IV Axis I Disorders (SCID I) and Axis II Disorders (SCID II). Clinical Psychology and Psychotherapy, 18(1), 75-79. doi:10.1002/cpp.693

López-Pelayo, H., Batalla, A., Balcells, M. M., Colom, J., \& Gual, A. (2015). Assessment of cannabis use disorders: A systematic review of screening and diagnostic instruments. Psychological Medicine, 45(6), 1121-1133. doi:http://dx.doi.org/10.1017/S0033291714002463

Loue, S. (2012). Ethical issues in a study of bipolar disorder and HIV risk among AfricanAmerican men who have sex with men: Case study in the ethics of mental health research. 
Journal of Nervous and Mental Disease, 200(3), 236-241.

doi:http://dx.doi.org/10.1097/NMD.0b013e318247cb43

Lovibond, S. H., \& Lovibond, P. F. (1995). Manual for the Depression Anxiety Stress Scales.

( $2^{\text {nd }}$ ed.). Sydney: Psychology Foundation. https://doi.org/10.1037/t39835-000

Low, Y., Goforth, H. W., Omonuwa, T., Preud'homme, X., Edinger, J., \& Krystal, A. (2012).

Comparison of polysomnographic data in age-, sex- and Axis I psychiatric diagnosis

matched HIV-seropositive and HIV-seronegative insomnia patients. Clinical

Neurophysiology, 123, 2402-2405. doi:10.1016/j.clinph.2012.05.004

Low, Y., Goforth, H., Preud'homme, X., Edinger, J., \& Krystal, A. (2014). Insomnia in HIVinfected patients: Pathophysiologic implications. AIDS Review, 16, 3-13.

Lu, M., Safren, S. A., Skolnik, P. R., Rogers, W. H., Coady, W., Hardy, H., \& Wilson, I. B. (2008). Optimal recall period and response task for self-reported HIV medication adherence. AIDS and Behavior, 12(1), 86-94. doi:http://dx.doi.org/10.1007/s10461-007$9261-4$

Maich, K. H. G., Lachowski, A. M., \& Carney, C. E. (2018). Psychometric properties of the Consensus Sleep Diary in those with insomnia disorder. Behavioral Sleep Medicine, 16(2), 117-134. https://doi.org/10.1080/15402002.2016.1173556

Manber, R., \& Carney, C. E. (2015). Treatment plans and interventions for insomnia: A case formulation approach. New York: Guilford.

Manber, R., Edinger, J. D., Gress, J. L., Pedro-Salcedo, M., Kuo, T. F., \& Kalista, T. (2008). Cognitive behavioral therapy for insomnia enhances depression outcome in patients with comorbid major depressive disorder and insomnia. Sleep, 31(4), 489495. https://doi.org/10.1093/sleep/31.4.489 
Manber, R., Friedman, L., Siebern, A. T., Carney, C., Edinger, J., Epstein, D., ... Karlin, B. E. (2014). Cognitive behavioral therapy for insomnia in veterans: Therapist manual.

Washington, DC: US Department of Veterans Affairs.

Martínez, M. P., Miró, E., Sánchez, A. I., Díaz-Piedra, C., Cáliz, R., Vlaeyen, J. W. S., \& BuelaCasal, G. (2014). Cognitive-behavioral therapy for insomnia and sleep hygiene in fibromyalgia: A randomized controlled trial. Journal of Behavioral Medicine, 37(4), 683697. doi:http://dx.doi.org/10.1007/s10865-013-9520-y

Matthews, K. A., Patel, S. R., Pantesco, E. J., Buysse, D. J., Kamarck, T. W., Lee, L., \& Hall, M. M. (2018). Similarities and differences in estimates of sleep duration by polysomnography, actigraphy, diary, and self-reported habitual sleep in a community sample. Sleep Health, 4(1), 96-103. doi:https://doi.org/10.1016/j.sleh.2017.10.011

McCall, C., \& McCall, W. V. (2012). Comparison of actigraphy with polysomnography and sleep logs in depressed insomniacs. Journal of Sleep Research, 21(1), 122-127. doi: 10.1111/j.1365-2869.2011.00917.x

Miller, W. R., \& Rollnick, S. (2002). Motivational interviewing: Preparing people for change. New York: Guilford.

Moeller, A. A., Oechsner, M., Backmund, H. C., Popescu, M., Emminger, C., \& Holsboer, F. (1991). Self-reported sleep quality in HIV infection: Correlation to the stage of infection and zidovudine therapy. Journal of Acquired Immune Deficiency Syndromes, 4(10), 10001003.

Morgan, K. (2012). The epidemiology of sleep. In C. M. Morin \& C. A. Espie (Eds.), The Oxford handbook of sleep and sleep disorders (pp. 303-323). New York: Oxford. doi:http://dx.doi.org/10.1093/oxfordhb/9780195376203.013.0016 
Morgan, K., \& Clarke, D. (1997). Risk factors for late-life insomnia in a representative general practice sample. British Journal of General Practice, 47(416): 166-169.

Morin, C. M. (1993). Insomnia: Psychological assessment and management. New York: Guilford.

Morin, C. M., Bélanger, L., LeBlanc, M., Ivers, H., Savard, J., Espie, C. A., ... Grégoire, J.-P. (2009). The natural history of insomnia: A population-based 3-year longitudinal study. Archives of Internal Medicine, 169(5), 447-453. doi:10.1001/archinternmed.2008.610

Morin, C. M., Belleville, G., Bélanger, L., \& Ivers, H. (2011). The Insomnia Severity Index: Psychometric indicators to detect insomnia cases and evaluate treatment response. Sleep: Journal of Sleep and Sleep Disorders Research, 34(5), 601-608. https://doi.org/10.1093/sleep/34.5.601

Morin, C. M., \& Benca, R. (2012). Chronic insomnia. The Lancet, 379, 1129-1141. doi:http://dx.doi.org/10.1016/S0140-6736(11)60750-2

Morin, C. M., Colecchi, C., Stone, J., Sood, R., \& Brink, D. (1999). Behavioral and pharmacological therapies for late-life insomnia: A randomized controlled trial. Journal of the American Medical Association, 281(11), 991-999. https://doi.org/10.1001/jama.281.11.991

Morin, C. M., \& Jarrin, D. C. (2013). Epidemiology of insomnia: Prevalence, course, risk factors, and public health burden. Sleep Medicine Clinics, 8(3), 281-297. doi:http://dx.doi.org/10.1016/j.jsmc.2013.05.002

Morin, C. M., Kowatch, R. A., Barry, T., \& Walton, E. (1993). Cognitive-behavior therapy for late-life insomnia. Journal of Consulting and Clinical Psychology, 61, 137-146. doi:http://dx.doi.org/ 10.1037/0022-006X.61.1.137 
Morin, C. M., LeBlanc, M., Bélanger, L., Ivers, H., Mérette, C., \& Savard, J. (2011). Prevalence of insomnia and its treatment in Canada. Canadian Journal of Psychiatry, 56(9), 540-548. https://doi.org/10.1177/070674371105600905

Morin, C. M., Vallières, A., \& Ivers, H. (2007). Dysfunctional beliefs and attitudes about sleep (DBAS): Validation of a brief version (DBAS-16). Sleep, 30(11), 1547-1554. https://doi.org/10.1093/sleep/30.11.1547

Natale, V., Léger, D., Bayon, V., Erbacci, A., Tonetti, L., Fabbri, M., \& Martoni, M. (2015). The Consensus Sleep Diary: Quantitative criteria for primary insomnia diagnosis. Psychosomatic Medicine, 77(4), 413-418. https://doi.org/10.1097/PSY.0000000000000177

Nokes, K. M., \& Kendrew, J. (2001). Correlates of sleep quality in persons with HIV disease. Journal of the Association of Nurses in AIDS Care, 12, 17-22. https://doi.org/10.1016/S1055-3290(06)60167-2

Norman, S. E., Chediak, A. D., Freeman, C., Kiel, M., Mendez, A., Duncan, R., ... Nolan, B. (1992). Sleep disturbances in men with asymptomatic human immunodeficiency (HIV) infection. Sleep, 15(2), 150-155. https://doi.org/10.1093/sleep/15.2.150

Norell-Clarke, A., Jansson-Fröjmark, M., Tillfors, M., Holländare, F., \& Engström, I. (2015). Group cognitive behavioural therapy for insomnia: Effects on sleep and depressive symptomatology in a sample with comorbidity. Behaviour Research and Therapy, 74, 8093. https://doi.org/10.1016/j.brat.2015.09.005

Nourbakhsh, M. R., \& Ottenbacher, K. J. (1994). The statistical analysis of single-subject data: A comparative examination. Physical Therapy, 74(8), 80-88.

https://doi.org/10.1093/ptj/74.8.768 
O’Cleirigh, C., Safren, S. A., Taylor, S. W., Goshe, B. M., Bedoya, C. A., Marquez, S. M., .. . Shipherd, J. C. (2019). Cognitive behavioral therapy for trauma and self-care (CBT-TSC) in men who have sex with men with a history of childhood sexual abuse: A randomized controlled trial. AIDS and Behavior, 1-11. doi:http://dx.doi.org.ezproxy.lib.ryerson.ca/10.1007/s10461-019-02482-z

Ohayon, M. M., (2002). Epidemiology of insomnia: What we know and what we still need to learn. Sleep Medicine Reviews, 6, 97-111. https://doi.org/10.1053/smrv.2002.0186

Ohayon, M. M. (2007). Insomnia: A ticking clock for depression? Journal of Psychiatric Research, 41, 893-894. https://doi.org/10.1016/j.jpsychires.2007.07.008

Ohayon, M. M., \& Reynolds, C. F., III. (2009). Epidemiological and clinical relevance of insomnia diagnosis algorithms according to the DSM-IV and the International Classification of Sleep Disorders (ICSD). Sleep Medicine, 10(9), 952-960. doi:http://dx.doi.org/10.1016/j.sleep.2009.07.008

Ohayon, M. M., \& Roth, T. (2003). Place of chronic insomnia in the course of depressive and anxiety disorders. Journal of Psychiatric Research, 37, 9-15. https://doi.org/10.1016/S0022-3956(02)00052-3

Okajima, I., Komada, Y., \& Inoue, Y. (2011). A meta-analysis on the treatment effectiveness of cognitive behavioral therapy for primary insomnia. Sleep and Biological Rhythms, 9(1), 24-34. https://doi.org/10.1111/j.1479-8425.2010.00481.x

Pala, A. N., \& Steca, P. (2015). Illness perceptions and coping strategies among individuals diagnosed with HIV. Journal of Behavioral Medicine, 38(4), 620-631. doi:http://dx.doi.org/10.1007/s10865-015-9639-0 
Papas, R. K., Sidle, J. E., Martino, S., Baliddawa, J. B., Songole, R., Omolo, O. E., . . Maisto, S. A. (2010). Systematic cultural adaptation of cognitive-behavioral therapy to reduce alcohol use among HIV-infected outpatients in western Kenya. AIDS and Behavior, 14(3), 669-678. doi:http://dx.doi.org.ezproxy.lib.ryerson.ca/10.1007/s10461-009-9647-6

Parrino, L., Ferri, R., Bruni, O., \& Terzano, M. G. (2012). Cyclic alternating pattern (CAP): The marker of sleep instability. Sleep Medicine Reviews, 16(1), 27-45. https://doi.org/10.1016/j.smrv.2011.02.003

Parsons, J. T., John, S. A., Millar, B. M., \& Starks, T. J. (2018). Testing the efficacy of combined motivational interviewing and cognitive behavioral skills training to reduce methamphetamine use and improve HIV medication adherence among HIV-positive gay and bisexual men. AIDS and Behavior, 22(8), 2674-2686. doi:http://dx.doi.org.ezproxy.lib.ryerson.ca/10.1007/s10461-018-2086-5

Patel, S. R., Malhotra, A., Gao, X., Hu, F. B., Neuman, M. I., \& Fawzi, W. W. (2012). A prospective study of sleep duration and pneumonia risk in women. Sleep, 35(1), 97-101. https://doi.org/10.5665/sleep.1594

Patil, S. P., Schneider, H., Schwartz, A. R., \& Smith, P. L. (2007). Adult obstructive sleep apnea: Pathophysiology and diagnosis. Chest, 132(1), 325-337. https://doi.org/10.1378/chest.070040

Perlis, M. L., Giles, D. E., Mendelson, W. B., Bootzin, R. R., \& Wyatt, J. K. (1997). Psychophysiological insomnia: The behavioural model and a neurocognitive perspective. Journal of Sleep Research, 6(3), 179-188. https://doi.org/10.1046/j.13652869.1997.00045.x 
Phillips, K. D., Moneyham, L., Murdaugh, C., Boyd, M. R., Tavakoli, A., Jackson, K., \& Vyavaharkar, M. (2005). Sleep disturbance and depression as barriers to adherence. Clinical Nursing Research, 14(3), 273-293. https://doi.org/10.1177/1054773805275122

Phillips, K. D., \& Skelton, W. D. (2001). Effects of individualized acupuncture on sleep quality in HIV disease. JANAC: Journal of the Association of Nurses in AIDS Care, 12(1), 27-39. doi:http://dx.doi.org/10.1016/S1055-3290(06)60168-4

Pilcher, C. D., Tien, H. C., Eron, J. J., Vernazza, P. L., Leu, S.-Y., Stewart, P. W., ... Cohen, M. S. (2004). Brief but efficient: Acute HIV infection and the sexual transmission of HIV. The Journal of Infectious Diseases, 189, 1785-1792. https://doi.org/10.1086/386333

Prochaska, J. O., Redding, C. A., \& Evers, K. E. (2015). The transtheoretical model and stages of change. In K. Glanz, B. K. Rimer, \& K. "V." Viswanath (Eds.), Health behavior: Theory, research, and practice (pp. 125-148). San Francisco, CA: Jossey-Bass.

Prowse, P. T., Meadows, G., \& Enticott, J. (2015). An exploratory study into the effectiveness of fidelity scales in the delivery of mindfulness-based cognitive therapy. Mindfulness, 6(6), 1401-1410. https://doi.org/10.1007/s12671-015-0412-9

Public Health Agency of Canada. (2015). Summary: Estimates of HIV incidence, prevalence and proportion undiagnosed in Canada, 2014. Retrieved from http://healthycanadians.gc.ca/publications/diseases-conditions-maladies-affections/hivaids-estimates-2014-vih-sida-estimations/index-eng.php

Public Health Agency of Canada. (2016). Section 5-8: Canadian guidelines on sexually transmitted infections - management and treatment of specific infections - human immunodeficiency virus infections. Retrieved from https://www.canada.ca/en/publichealth/services/infectious-diseases/sexual-health-sexually-transmitted-infections/canadian- 
guidelines/sexually-transmitted-infections/canadian-guidelines-sexually-transmittedinfections-36.html

Public Health Agency of Canada. (2018). Summary: Estimates of HIV incidence, prevalence, and Canada's progress on meeting the 90-90-90 HIV targets, 2016. Retrieved from https://www.canada.ca/content/dam/phac-aspc/documents/services/publications/diseasesconditions/summary-estimates-hiv-incidence-prevalence-canadas-progress-90-90-90/pubeng.pdf

Qaseem, A., Kansagara, D., Forciea, M. A., Cooke, M., \& Denberg, T. D. for the Clinical Guidelines Committee of the American College of Physicians. (2016). Management of chronic insomnia disorder in adults: A clinical practice guideline from the American College of Physicians. Annals of Internal Medicine, 165(2), 1-15. doi:10.7326/M15-2175

Radloff, L. S. (1977). The CES-D scale: A self-report depression scale for research in the general population. Applied Psychological Measurement, 1, 385-401. https://doi.org/10.1177/014662167700100306

Reid, S., \& Dwyer, J. (2005). Insomnia in HIV infection: A systematic review of prevalence, correlates, and management. Psychosomatic Medicine, 67(2), 260-269. doi:http://dx.doi.org/10.1097/01.psy.0000151771.46127.df

Riemann, D., Spiegelhalder, K., Feige, B., Voderholzer, U., Berger, M., Perlis, M., \& Nissen, C. (2010). The hyperarousal of insomnia: A review of the concept and its evidence. Sleep Medicine Reviews, 14(1), 19-31. https://doi.org/10.1016/j.smrv.2009.04.002

Robbins, J. L., Phillips, K. D., Dudgeon, W. D., \& Hand, G. A. (2004). Physiological and psychological correlates of sleep in HIV infection. Clinical Nursing Research, 13(1), 3352. https://doi.org/10.1177/1054773803259655 
Robey, R. R. (2004). A five-phase model for clinical-outcome research. Journal of Communication Disorders, 37(5), 401-411. doi:http://dx.doi.org/ 10.1016/j.jcomdis.2004.04.003

Robotham, D. (2011). Sleep as a public health concern: Insomnia and mental health. Journal of Public Mental Health, 10(4), 234-237. doi:http://dx.doi.org/10.1108/17465721111188250

Rossman, G. B., \& Wilson, B. L. (1985). Numbers and words: Combining quantitative and qualitative methods in a single large-scale evaluation study. Evaluation Review, 9(5), 627643. https://doi.org/10.1177/0193841X8500900505

Rubin, D. B. (1996). Multiple imputation after 18+ years. Journal of the American Statistical Association, 91(434), 473-489. https://doi.org/10.1080/01621459.1996.10476908

Rubinstein, M. L., \& Selwyn, P. A. (1998). High prevalence of insomnia in an outpatient population with HIV infection. Journal of Acquired Immune Deficiency Syndromes and Human Retrovirology, 19, 260-265. https://doi.org/10.1097/00042560-199811010-00008

Rutledge, C. M., La Guardia, A. C., \& Bluestein, D. (2013). Predictors of self-efficacy for sleep in primary care. Journal of Clinical Nursing, 22(9-10), 1254-1261. doi:http://dx.doi.org/10.1111/jocn.12005

Saberi, P., Neilands, T. B., \& Johnson, M. O. (2011). Quality of sleep: Associations with antiretroviral nonadherence. AIDS Patient Care and STDs, 25(9), 517-524. https://doi.org/10.1089/apc.2010.0375

Safren, S. A., Bedoya, C. A., O'Cleirigh, C., Biello, K. B., Pinkston, M. M., Stein, M. D., .. . Mayer, K. H. (2016). Cognitive behavioural therapy for adherence and depression in patients with HIV: A three-arm randomised controlled trial. Lancet HIV, 3(11), e529-e538. doi:http://dx.doi.org.ezproxy.lib.ryerson.ca/10.1016/S2352-3018(16)30053-4 
Samji, H., Cescon, A., Hogg, R. S., Modur, S. P., Althoff, K. N., Buchacz, K., ... Gange, S. J. (2013). Closing the gap: Increases in life expectancy among treated HIV-positive individuals in the United States and Canada. PLOS ONE, 8(12), 1-8. https://doi.org/10.1371/journal.pone.0081355

Saunders, D. S., \& Burgoyne, R. W. (2002). Evaluating health-related wellbeing outcomes among outpatient adults with human immunodeficiency virus infection in the HAART era. International Journal of STD \& AIDS, 13(10), 683-690.

doi:http://dx.doi.org/10.1258/095646202760326435

Savard, J., Laberge, B., Gauthier, J. G., Ivers, H., \& Bergeron, M. G. (1998). Evaluating anxiety and depression in HIV-infected patients. Journal of Personality Assessment, 71(3), 349367. https://doi.org/10.1207/s15327752jpa7103_5

Savard, J., Laroche, L., Simard, S., Ivers, H., \& Morin, C. M. (2003). Chronic insomnia and immune functioning. Psychosomatic Medicine, 65(2), 211-221. https://doi.org/10.1097/01.PSY.0000033126.22740.F3

Savard, J., Savard, M.-H., \& Morin, C. M. (2010). Insomnia. In M. M. Antony \& D. H. Barlow (Eds.), Handbook of assessment and treatment planning for psychological disorders (pp. 633-669). New York: Guilford.

Savard, M.-H., Savard, J., Simard, S., \& Ivers, H. (2005). Empirical validation of the Insomnia Severity Index in cancer patients. Psycho-Oncology, 14(6), 429-441. doi:http://dx.doi.org/10.1002/pon.860

Schutte-Rodin, S., Broch, L., Buysse, D., Dorsey, C., \& Sateia, M. (2008). Clinical guideline for the evaluation and management of chronic insomnia in adults. Journal of Clinical Sleep Medicine, 4(5), 487-504. 
Schwartz, D. R., \& Carney, C. E. (2012). Mediators of cognitive-behavioral therapy for insomnia: A review of randomized controlled trials and secondary analysis studies. Clinical Psychology Review, 32(7), 664-675. doi:http://dx.doi.org/10.1016/j.cpr.2012.06.006

Seay, J. S., McIntosh, R., Fekete, E. M., Fletcher, M. A., Kumar, M., Schneiderman, N., \& Antoni, M. H. (2013). Self-reported sleep disturbance is associated with lower CD4 count and 24-h urinary dopamine levels in ethnic minority women living with HIV. Psychoneuroendocrinology, 38(11), 26472653. https://doi.org/10.1016/j.psyneuen.2013.06.022

Shacham, E., Rosenburg, N., Onen, N.F., Donovan, M. F., \& Overton, E. T. (2015). Persistent HIV-related stigma among an outpatient US clinic population. International Journal of STD \& AIDS, 26(4), 243-250. https://doi.org/10.1177/0956462414533318

Sheehan, D. V., Lecrubier, Y., Sheehan, K. H., Amorim, P., Janavs, J., Weiller, E., . . Dunbar, G. C. (1998). The Mini-International Neuropsychiatric Interview (M.I.N.I): The development and validation of a structured diagnostic psychiatric interview for DSM-IV and ICD-10. Journal of Clinical Psychiatry, 59, 22-33. https://doi.org/10.1037/t18597-000

Sidani, S. (2015). Health intervention research: Understanding research design and methods. Thousand Oaks, CA: Sage. https://doi.org/10.4135/9781473910140

Simoni, J. M., Safren, S. A., Manhart, L. E., Lyda, K., Grossman, C. I., Rao, D., . . Wilson, I. B. (2011). Challenges in addressing depression in HIV research: Assessment, cultural context, and methods. AIDS and Behavior, 15(2), 376-388. doi:http://dx.doi.org.ezproxy.lib.ryerson.ca/10.1007/s10461-010-9836-3 
Sivertsen, B., Omvik, S., Pallesen, S., Bjorvatn, B., Havik, O. E., Kvale, G., . . Nordhus, I. H. (2006). Cognitive behavioral therapy vs zopiclone for treatment of chronic primary insomnia in older adults: A randomized controlled trial. Journal of the American Medical Association, 295(24), 2851-2858. doi:http://dx.doi.org/10.1001/jama.295.24.2851

Sivertsen, B., Salo, P., Mykletun, A., Hysing, M., Pallesen, S., Krokstad, S., ... Øverland, S. (2012). The bidirectional association between depression and insomnia: The HUNT study. Psychosomatic Medicine, 74(7), 758-765. https://doi.org/10.1097/PSY.0b013e3182648619

Smith, M. T., Huang, M. I., \& Manber, R. (2005). Cognitive behavior therapy for chronic insomnia occurring within the context of medical and psychiatric disorders. Clinical Psychology Review, 25, 559-592. https://doi.org/10.1016/j.cpr.2005.04.004

Smith, M. T., \& Perlis, M. L. (2006). Who is a candidate for cognitive-behavioral therapy for insomnia? Health Psychology, 25(1), 15-19. https://doi.org/10.1037/0278-6133.25.1.15

Stanley, S., Sethuramalingam, V., \& Sathia, S. (2014). Quality of life correlates in HIV-positive people in a city in south India. Journal of HIV/AIDS \& Social Services, 13(4), 337-352. doi:http://dx.doi.org/10.1080/15381501.2013.826612

Sunnhed, R., \& Jansson-Fröjmark, M. (2015). Cognitive arousal, unhelpful beliefs and maladaptive sleep behaviors as mediators in cognitive behavior therapy for insomnia: A quasi-experimental study. Cognitive Therapy Research, 39, 841-852. doi:10.1007/s10608015-9698-0

Sweetman, A., Lack, L., Lambert, S., Gradisar, M., \& Harris, J. (2017). Does comorbid obstructive sleep apnea impair the effectiveness of cognitive and behavioral therapy for 
insomnia? Sleep Medicine, 39, 38-46.

doi:http://dx.doi.org.ezproxy.lib.ryerson.ca/10.1016/j.sleep.2017.09.003

Taibi, D. M. (2013). Sleep disturbances in persons living with HIV. Journal of the Association of Nurses in AIDS Care,24(1), S72-S85. https://doi.org/10.1016/j.jana.2012.10.006

Tariq, S., \& Woodman, J. (2013). Using mixed methods in health research. Journal of the Royal Society of Medicine Short Reports, 4(6). doi:http://10.1177/2042533313479197

Taylor, D., Carlyle, J., McPherson, S., Rost, F., Thomas, R., \& Fonagy, P. (2012). Tavistock Adult Depression Study (TADS): A randomised controlled trial of psychoanalytic psychotherapy for treatment-resistant/treatment-refractory forms of depression. $B M C$ Psychiatry, 12 doi:http://dx.doi.org/10.1186/1471-244X-12-60

Taylor, D. J., Lichstein, K. L., \& Durrence, H. H. (2003). Insomnia as a health risk factor. Behavioral Sleep Medicine, 1(4), 227-247. doi:http://dx.doi.org/10.1207/S15402010BSM0104_5

Taylor, D. J., \& Pruiksma, K. E. (2014). Cognitive and behavioural therapy for insomnia (CBTI) in psychiatric populations: A systematic review. International Review of Psychiatry, 26(2), 205-213. doi:http://dx.doi.org/10.3109/09540261.2014.902808

Thomas, P. W., Thomas, S., Kersten, P., Jones, R., Nock, A., Slingsby, V., . . Hillier, C. (2010). Multi-centre parallel arm randomised controlled trial to assess the effectiveness and costeffectiveness of a group-based cognitive behavioural approach to managing fatigue in people with multiple sclerosis. BMC Neurology, 10(43), 1-12. doi:http://dx.doi.org/10.1186/1471-2377-10-43

Trafton, J. A., Sorrell, J. T., Holodniy, M., Pierson, H., Link, P., Combs, A., \& Israelski, D. (2012). Outcomes associated with a cognitive-behavioral chronic pain management 
program implemented in three public HIV primary care clinics. The Journal of Behavioral Health Services \& Research, 39(2), 158-73. https://doi.org/10.1007/s11414-011-9254-y

Treisman, G., \& Angelino, A. (2007). Interralation between psychiatric disorders and the prevention and treatment of HIV infection. Clinical Infectious Diseases, 45, S313-S317. https://doi.org/10.1086/522556

U.S. Department of Health and Human Services. (2017). Guidelines for the use of antiretroviral agents in adults and adolescents with HIV. Retrieved from https://aidsinfo.nih.gov/guidelines/html/1/adult-and-adolescent-arv/10/initiation-ofantiretroviral-therapy

Uthman, O. A., Magidson, J. F., Safren, S. A., \& Nachega, J. B. (2014). Depression and adherence to antiretroviral therapy in low-, middle-, and high-income countries: A systematic review and meta-analysis. Current HIV/AIDS Reports, 11(3), 291-307. doi: $10.1007 / \mathrm{s} 11904-014-0220-1$

Villarruel, A. M., Gal, T. L., Eakin, B. L., Wilkes, A., \& Herbst, J. H. (2010). From research to practice: The importance of community collaboration in the translation process. Research and Theory for Nursing Practice, 24(1), 25-34.

Voss, J. G., Portillo, C. J., Holzemer, W. L., \& Dodd, M. J. (2007). Symptom cluster of fatigue and depression in HIV/AIDS. Journal of Prevention \& Intervention in the Community, 33(1-2), 19-34. doi:http://dx.doi.org/10.1300/J005v33n01_03

Wampold, B. E. (2015). How important are the common factors in psychotherapy? An update. World Psychiatry, 14(3), 270-277. https://doi.org/10.1002/wps.20238 
Wang, M., Wang, S., \& Tsai, P. (2005). Cognitive behavioural therapy for primary insomnia: A systematic review. Journal of Advanced Nursing, 50(5), 553-564. doi:http://dx.doi.org/10.1111/j.1365-2648.2005.03433.x

Ware, J. E., \& Sherbourne, C. D. (1992). The MOS 36-item Short-Form Health Survey (SF-36): I. Conceptual framework and item selection. Medical Care, 30(6), 473-483. https://doi.org/10.1097/00005650-199206000-00002

Watson, N. F., Badr, M. S., Belenky, G., Bliwise, D. L., Buxton, O. M., Buysse, D., . . Heald, J. L. (2015). Recommended amount of sleep for a healthy adult: A joint consensus statement of the American Academy of Sleep Medicine and Sleep Research Society. Sleep, 38(6), 843-844. https://doi.org/10.5664/jcsm.4758

Whetten, K., Reif, S., Whetten, R., \& Murphy-MacMillan, L. K. (2008). Trauma, mental health, distrust, and stigma among HIV-positive persons: Implications for effective care. Psychosomatic Medicine, 70, 531-538. https://doi.org/10.1097/PSY.0b013e31817749dc

White, J., Darko, D. F., Brown, S. J., Miller, J. C., Hayduk, R., Kelly, T., \& Mitler, M. M. (1995). Early central nervous system response to HIV infection: Sleep distortion and cognitive-motor decrements. AIDS, 9(9), 1043-1050. https://doi.org/10.1097/00002030199509000-00009

White, O. R. (1974). The "split middle" a "quickie" method of trend estimation. University of Washington, Experimental Education Unit, Child Development and Mental Retardation Center.

Wibbeler, T., Reichelt, D., Husstedt, I.-W., \& Evers, S. (2012). Sleepiness and sleep quality in patients with HIV infection. Journal of Psychosomatic Research, 72, 439-442. https://doi.org/10.1016/j.jpsychores.2012.03.003 
Wiegand, M., Möller, A. A., Schreiber, W., Krieg, J., Fuchs, D., Wachter, H., \& Holsboer, F. (1991). Nocturnal sleep EEG in patients with HIV infection. European Archives of Psychiatry and Clinical Neuroscience, 240(3), 153-

158. https://doi.org/10.1007/BF02190756

World Health Organization. (2007). WHO case definitions of HIV for surveillance and revised clinical staging and immunological classification of HIV-related disease in adults and children. Retrieved from http://www.who.int/hiv/pub/guidelines/HIVstaging150307.pdf

World Health Organization. (2010). The alcohol, smoking and substance involvement screening test (ASSIST): Manual for use in primary care. Geneva: World Health Organization.

World Health Organization. (2018). HIV/AIDS. Retrieved from https://www.who.int/en/newsroom/fact-sheets/detail/hiv-aids

Zanarini, M. C., \& Frankenburg, F. R. (2001). Attainment and maintenance of reliability of axis I and II disorders over the course of a longitudinal study. Comprehensive Psychiatry, 42(5), 369-374. doi:10.1053/comp.2001.24556

Zhang, B., \& Wing, Y. (2006). Sex differences in insomnia: A meta-analysis. Sleep, 29(1), 8593. https://doi.org/10.1093/sleep/29.1.85

Zigmond, A. S., \& Snaith, R. P. (1983). The Hospital Anxiety and Depression Scale. Acta Psychiatrica Scandinavica, 67(6), 361-370. https://doi.org/10.1111/j.16000447.1983.tb09716.x 


\section{Glossary of Abbreviations}
AIDS Acquired immune deficiency syndrome
cART Combination antiretroviral therapy
CBT-I Cognitive behavioural therapy for insomnia
CD4+ Cluster of differentiation 4 (applies to CD3+ and CD8+ also)
COPD Chronic obstructive pulmonary disease
DSM Diagnostic and Statistical Manual of Mental Disorders
HIV Human immunodeficiency virus
ISI Insomnia Severity Index
KTE Knowledge transfer and exchange
NK Natural killer
OSA Obstructive sleep apnea
PWH People living with HIV
REM Rapid eye movement 